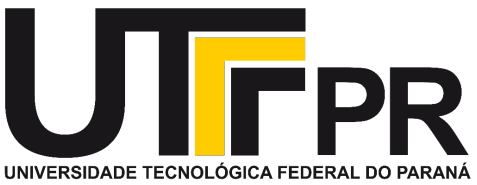

FEDERAL UNIVERSITY OF TECHNOLOGY - PARANÁ

DEPARTMENT OF MECHANICAL ENGINEERING

POSTGRADUATE PROGRAM IN MECHANICAL AND MATERIALS

ENGINEERING

YAMID JOSÉ GARCÍA BLANCO

VISUALIZATION OF VISCOPLASTIC FLUID FLOW IN AN ABRUPT CONTRACTION USING PARTICLE IMAGE VELOCIMETRY

MASTER THESIS

CURITIBA 


\section{VISUALIZATION OF VISCOPLASTIC FLUID FLOW IN AN ABRUPT CONTRACTION USING PARTICLE IMAGE VELOCIMETRY}

MSc thesis presented to the Postgraduate Programme in Mechanical and Materials Engineering - PPGEM from the Federal University of Technology - Paraná, as partial fulfilment of the requirements for Master degree in Mechanical Engineering.

Supervisor: Prof. Dr. Admilson T. Franco Co-supervisor: Prof. Dr. Eduardo M. Germer 
Dados Internacionais de Catalogação na Publicação

García Blanco, Yamid José

Visualization of viscoplastic fluid flow in an abrupt contraction using particle image velocimetry [recurso eletrônico] / Yamid José García Blanco. -- 2019.

1 arquivo texto (145f.) : PDF ; 5,48 MB.

Modo de acesso: World Wide Web

Título extraído da tela de título (visualizado em 21 fev. 2020)

Texto em inglês com resumo em português

Dissertação (Mestrado) - Universidade Tecnológica Federal do Paraná. Programa de Pós-graduação em Engenharia Mecânica e de Materiais, Curitiba, 2019

Bibliografia: f. 128-134.

1. Engenharia mecânica - Dissertações. 2. Escoamento. 3. Materiais viscoelásticos. 4. Velocimetria por imagem de partícula. 5. Fluidos. 6. Plasticidade. 7. Fluidos newtonianos. 8. Dinâmica dos fluidos. I. Franco, Admilson Teixeira. II. Germer, Eduardo Matos. III. Universidade Tecnológica Federal do Paraná. Programa de Pós-graduação em Engenharia Mecânica e de Materiais. IV. Título.

CDD: Ed. $23-620.1$

Biblioteca Central da UTFPR, Câmpus Curitiba

Bibliotecário: Adriano Lopes CRB-9/1429 


\section{TERMO DE APROVAÇÃO DE DISSERTAÇÃO №368}

A Dissertação de Mestrado intitulada: Visualization of viscoplastic fluid flow in an abrupt contraction using Particle Image Velocimetry, defendida em sessão pública pelo Candidato Yamid José García Blanco, no dia 22 de novembro de 2019, foi julgada para a obtenção do título de Mestre em Engenharia, área de concentração: Engenharia Térmica, linha de pesquisa: Mecânica dos Fluidos e aprovada em sua forma final, pelo Programa de Pós-Graduação em Engenharia Mecânica e de Materiais - PPGEM.

\section{BANCA EXAMINADORA:}

Prof. Dr. Admilson Teixeira Franco - Presidente - UTFPR

Prof. Dr. Cezar Otaviano Ribeiro Negrão - UTFPR

Prof. Dr. Diogo Berta Pitz - UFPR

Prof. Dr. Guilherme Henrique Fiorot - UFRGS

A via original deste documento encontra-se arquivada na Secretaria do Programa, contendo a assinatura da Coordenação após a entrega da versão corrigida do trabalho.

Curitiba, 22 de novembro de 2019. 


\section{ACKNOWLEDGEMENTS}

I aknowledge my mother, who gave me the most precious teaching, for her support and guidance. She has always been my motivation and has encouragement bestowed upon me.

To my father, who is no longer with us, but he gave me the necessary tools and lighted my way up here.

To my supervisor, Admilson T. Franco, and my co-supervisor, Eduardo M. Germer, for the opportunity, guidance, dedication, patience and confidence in my capacity.

The LabFlow trainees and employees, who helped me in the construction of the experimental setup and for the invaluable support in doing this work.

To all UTFPR personnel, who directly or indirectly supported me in carrying out this work. 
Beati hispani, qvibvs vivere bibere est Dichosos los hispanos, para los que vivir es beber 


\begin{abstract}
The viscoplastic fluid flow of Carbopol solutions is investigated in the following experimental study, for laminar and turbulent regime through an abrupt contraction with an aspect ratio of $\beta=1.85$. The pressure drop profile and the pressure drop coefficient at the contraction are obtained for different entrance Reynolds numbers, $R e_{D}^{\prime}$. The velocity vector fields are obtained for $R e_{D}^{\prime}$ between 1 and 10000 , using the Particle Image Velocimetry (PIV-2D). Mean velocity vector field and first-order turbulent quantities such as radial and axial velocity fluctuations are obtained, as well as centerline velocity behavior, vortex structures, and visualization of unyielded regions. It is observed that the pressure drop coefficient for viscoplastic fluid flow is dependent on the $R e_{D}^{\prime}$ for the laminar regime, and reaches a constant value for turbulent conditions, exhibiting good agreement with data available on the literature. The velocity profiles along the test section present lower values than those obtained for Newtonian fluids in both regimes and vary their behavior according to the rheological properties for each viscoplastic solution. Besides, the turbulent fluctuations approach the Newtonian behavior, with a slight difference for the radial profiles. The unyielded regions are deformed as the Reynolds number is increased and are replaced by vortex structures as the flow reaches transitional conditions, obtaining a variation of the effective diameter at the entrance of the contraction. The growth and changes of the vortex structure are studied using quantitative techniques based on the strain rate and the vorticity tensors, to gain accurate and better information about the behavior for these structures into the viscoplastic fluid flow. Finally, a relation was found for the entrance Reynolds number and the Reynolds number at the contraction plane, which is not dependent on the rheological parameters due to the high shear rates in this region.
\end{abstract}

Keywords: Viscoplastic fluid, experimental study, abrupt contraction, PIV. 


\section{RESUMO}

No presente estudo experimental analisamos as especificidades do escoamento de fluidos viscoplásticos, especialmente neste caso as soluções de Carbopol em regime laminar e turbulento através de uma contração abrupta com razão de $\beta=1.85$. O perfil e o coeficiente de queda de pressão na contração são obtidos a partir da entrada de diferentes números de Reynolds, $R e_{D}^{\prime}$. Os campos vetoriais de velocidade instantâneos são obtidos para $R e_{D}^{\prime}$ entre 1 e 10000 , usando a velocimetria por imagem de partícula (PIV-2D), verificando campos vetoriais de velocidade média e quantidades turbulentas de primeira ordem, tais como flutuações de velocidade radial e axial, bem como comportamento da velocidade da linha central, estruturas de vórtices e visualização de regiões não cisalhadas. Observa-se que o coeficiente de queda de pressão para o escoamento de fluido viscoplástico depende diretamente do número de Reynolds no regime laminar e atinge um valor constante para condições turbulentas, apresentando boa concordância com os dados disponíveis na atual literatura. Os perfis de velocidade ao longo da seção de teste apresentam valores inferiores aos obtidos para fluidos Newtonianos em ambos regimes e variam seu comportamento de acordo com as propriedades reológicas de cada solução. Ademais verificam-se flutuações turbulentas similares ao comportamento Newtoniano, apenas com discreta diferença nos perfis radiais. Nota-se que as regiões não cisalhadas deformam-se com o aumento do número de Reynolds e são substituídas por estruturas de vórtice à medida que o escoamento atinge condições de transição, obtendo uma variação do diâmetro efetivo na entrada da contração. O desenvolvimento da estrutura do vórtice foi estudado usando técnicas quantitativas baseadas na taxa de deformação e nos tensores de vorticidade, obtendo assim informações mais precisas sobre seu comportamento no escoamento de fluidos viscoplásticos. Dessa forma, pode-se determinar uma relação entre o número de Reynolds de entrada e o número de Reynolds no plano da contração, independente dos parâmetros reológicos devido às altas taxas de cisalhamento nessa região.

Palavras-chave: Fluido viscoplástico, estudo experimental, contração abrupta, PIV. 


\section{LIST OF FIGURES}

Figure 1.1 - Basic parts and contractions of a drilling string. Contractions are indicated by red arrows. . . . . . . . . . . . . . . . 20

Figure 1.2 - Geometry of the flow through an abrupt circular contraction. . . . . . . . . 21

Figure 2.1 - Representation of unidirectional shearing flow. . . . . . . . . . . . . . . . 24

Figure 2.2 - Flow curves schema for Newtonian and non-Newtonian fluids. . . . . . . . 27

Figure 2.3 - Sketch of empirical curve-fitting models for viscoplastic fluids. . . . . . . . 29

Figure 2.4 - Force balance in a flow through a horizontal pipe. . . . . . . . . . . . 30

Figure 2.5 - Velocity distribution for laminar flow of a viscoplastic fluid in a pipe. . . . . 31

Figure 2.6 - Flow of viscoplastic fluid through a contraction. Points 1, 2, 3, and 4 presents the velocity profile changes along the contraction. . . . . . . . . . 36

Figure 2.7 - Experimental arrangement for particle image velocimetry and correlation process. . . . . . . . . . . . . . . . . 39

Figure 2.8 - Particle density in laser applications techniques: (a) low (PTV), (b) medium (PIV), (c) high particle density (LSV). . . . . . . . . . . . . . .

Figure 3.1 - Local friction coefficient as a function of the Reynolds number for different dimensionless yield stress. (a) Entrance flow with a uniform profile. (b) 4:1 abrupt contraction. . . . . . . . . . . . . . . . . 44

Figure 4.1 - Steady flow curve (a) and viscosity (b) behavior obtained for each fluid. Rheological data were obtained with a HAAKE-MARS III. . . . . . . . . . 51

Figure 4.2 - Different set of refractions index at the visualization box: (a) optical distortions caused by the curvature of the circular tube. (b) box filled with the same working fluid. (c) equal refraction indices for the circular tube and the working fluid. . . . . . . . . . . . . . . . 53

Figure 4.3 - Experimental apparatus: hydraulic system for tests performing. . . . . . . . 55

Figure 4.4 - Schema of the contraction test section with dimensions expressed in millimeters (mm). The test section is made of a solid acrylic piece, designed to fit with the flow-loop pipes at entrance and exit sections. . . . . . . . . .

Figure 4.5 - Laser and visualization system, the plane of the laser beam and the visualization plane of the camera are set in a perpendicular configuration. Red lines show the perpendicular configuration.

Figure 4.6 - Optical filters used for CMOS camera: (a) polarizer filter, commonly used for glass and polymer particles. (b) orange sharp cut filter for polymer particles coated by rodamine-B.

Figure 4.7 - Comparison of images obtained from the light scattered by: (a) HGS particles and (b) polyamida particles coated by rodamine-B. The images were taken from a Newtonian fluid flow. 
Figure 4.8 - Light scattering by glass particles in water, for different diameters: (a) $1 \mu \mathrm{m}$.

(b) $10 \mu \mathrm{m}$. (c) $30 \mu \mathrm{m} . \ldots \ldots$. . . . . . . . . . . . . . . . 62

Figure 4.9 - Model of a CMOS sensor . . . . . . . . . . . . . . . . . 63

Figure 4.10-Single frame techniques are shown at upper side, for: (a) single exposure.

(b) double exposure. (c) multiple exposure. At the bottom side are shown the two most common multiple frame techniques: (d) double frame with single exposure and (e) double frame with double exposure. The open circles indicate the particle position in the previous frame. . . . . . . . . . . . . .

Figure 4.11-Conceptual description of a double frame single exposure Particle Image Velocimetry. . . . . . . . . . . . . . . . . .

Figure 4.12-Linear digital signal processing model describing the functional relationship between two successively particle image frames. . . . . . . . . . . . . . .

Figure 4.13-Two-dimensional map for calculating the position of the peak correlation. Rows represent the $\mathrm{x}$-coordinate and columns the y-coordinate for the peak correlation point, and Max is the point at the maximum correlation value. . .

Figure 4.14-Correlation peak function (c) calculated for an interrogation area of $32 \times 32$ using a double frame (a) and (b) - single exposure method. Correlation made at $R e=24600$ for water flow. . . . . . . . . . . . . . . . . 68

Figure 4.15-Overlap method applied for a interrogation area. The area was overlapped $50 \%$ at the neighboring areas. Retrieved from Dynamics (2015). . . . . . .

Figure 4.16-Sampling interrogation area using: (a) central difference interrogation (CDI) scheme. (b) forward difference interrogation (FDI) scheme. . . . . . . . . . 70

Figure 4.17-Measuring of pressure along the flow-loop . . . . . . . . . . . . . . . 73

Figure 4.18-Process for image acquisition and post-processing steps with Dynamic Studio software. . . . . . . . . . . . . . . . . .

Figure 4.19-Experimental friction factor coefficients obtained for a circular section for each viscoplastic solutions. Experimental data are compared with the correlations of Malin (1998) for viscoplastic fluids, and Blasius (1913) correlation for Newtonian fluids.

Figure 5.1 - Pressure profiles along the flow-loop as a function of the dimensionless distance from the contraction $Z / D$ and the Reynolds number at the test section entrance, $R e_{D}^{\prime}$. Each viscoplastic solution is presented: (a) $\tau_{0}^{H}=$ $0.07 \mathrm{~Pa}(10 \mathrm{~W}),(\mathrm{b}) \tau_{0}^{H}=0.26 \mathrm{~Pa}(20 \mathrm{~W}),(\mathrm{c}) \tau_{0}^{H}=2.82 \mathrm{~Pa}(30 \mathrm{~W})$ and (d) $\tau_{0}^{H}=6.99 \mathrm{~Pa}(40 \mathrm{~W}) . \ldots \ldots \ldots \ldots \ldots$

Figure 5.2 - Pressure drop for each yield stress solution as a function of the Reynolds number at the entrance section. . . . . . . . . . . . . . .

Figure 5.3 - Pressure drop non-dimensionalized by the dynamic pressure for each viscoplastic solution characterized by $H e_{H B}$ as function of $R e_{D}^{\prime}$. Comparison with experimental studies performed for Newtonian fluid. 
Figure 5.4 - Pressure loss coefficient at the contraction in function of the Reynolds number at the entrance section. Experimental values were fit by K's method proposed by Hooper (1981). The laminar and turbulent trends are presented by dashed and dot-dashed lines.

Figure 5.5 - Comparison of the pressure loss coefficient correlations found in the present study with the correlations found in the literature for viscoplastic fluid flow.

Figure 5.6 - Mean statistic velocity vector field for laminar flow. Solution $30 \mathrm{~W} R e_{D}^{\prime}=10.88$ Figure 5.7 - Mean axial velocity map for laminar $R e_{D}^{\prime}$. a) and b) present the flow maps for $10 \mathrm{~W}$ fluid. c), d), e), and f) show the dimensionless axial velocity maps for $20 \mathrm{~W}$ fluid.

Figure 5.8 - Mean axial velocity map for laminar $R e_{D}^{\prime}$. a), b), and c) show the maps for 30W fluid. d), e), and f) present the maps for $40 \mathrm{~W}$ fluid.

Figure 5.9 - Mean radial velocity map for laminar $R e_{D}^{\prime}$. a) and b) are the flow maps obtained for $10 \mathrm{~W}$ fluid. c), d), e), and f) are the dimensionless radial velocity maps for $20 \mathrm{~W}$ fluid.

Figure 5.10-Mean radial velocity distribution for laminar $R e_{D}^{\prime}$. a), b), and c) show the maps for $30 \mathrm{~W}$ fluid. d), e), and f) present the maps for $40 \mathrm{~W}$ fluid.

Figure 5.11-Mean axial velocity map distribution for turbulent $\operatorname{Re}_{D}^{\prime}$. a), b), and c) show the maps for $10 \mathrm{~W}$ fluid.

Figure 5.12-Mean radial velocity map for turbulent $\operatorname{Re}_{D}^{\prime}$. a), b), and c) show the maps for 10W fluid.

Figure 5.13-Mean axial velocity profiles for laminar regime: a) and b) for 10W; c), d), e) and f) for 20W. Red-dashed lines represent the central plug core size. . . . .

Figure 5.14-Mean axial velocity profiles in laminar regime: a), b), and c) shows the profiles for $30 \mathrm{~W}$; d), e), and f) for $40 \mathrm{~W}$. Red-dashed lines represent the central plug core size.

Figure 5.15-Dimensionless radial velocity profiles for laminar regime: a) and b) for 10W; c), d), e) and f) for $20 \mathrm{~W}$.

Figure 5.16-Dimensionless radial velocity profiles for laminar regime: a), b), and c) for $30 \mathrm{~W}$; d), e), and f) for $40 \mathrm{~W}$.

Figure 5.17-Comparison of the axial dimensionless velocity profiles between Newtonian and the viscoplastic fluids flow for the dimensionless axial position $Z / D=$ $-1.047, Z / D=-0.104$, and -0.039 .

Figure 5.18-Comparison of the radial dimensionless velocity profiles between Newtonian and the viscoplastic fluids flow for the dimensionless axial position $Z / D=$ -0.104 and -0.039 .

Figure 5.19-Mean velocity profiles for fluid $10 \mathrm{~W}\left(H e_{H B} \approx 0\right)$ at turbulent regime: a), b), and c) display the axial dimensionless velocity; d), e), and f) the radial velocity ratio. 
Figure 5.20-Comparison of the axial and radial velocity profiles for fluid 10W with Newtonian fluids for turbulent regime: a) and c) show the axial profile ratios for $R e_{D}^{\prime}=5000$; b) and d) display the radial velocity ratio for $R e_{D}^{\prime}=10000.105$

Figure 5.21-Axial dimensionless velocity along the centerline, $u_{C L} / \bar{U}$, as a function of the axial position $Z / D$, and the Reynolds number $R_{D}^{\prime}$. . . . . . . . 106

Figure 5.22-Turbulent intensities: axial and radial fluctuations for turbulent $R e_{D}^{\prime}$, plotted as a function of the dimensionless radial coordinate. . . . . . . . . . . . 108

Figure 5.23-Turbulent intensities: axial and radial fluctuations for turbulent $R e_{D}^{\prime}$, plotted as a function of the dimensionless radial coordinate. . . . . . . . . . . . . 109

Figure 5.24-Visualization at the corner of the contractions for different $R e_{D}^{\prime}$. The generation and growth of the vortex structures are displayed by vector maps for fluid 10W in a), b), c), d), e), and for fluid 20W in figures f), g), h), i), and j). Red dashed-lines delimited the unyielded regions. . . . . . . . . . . . . 112

Figure 5.25-Visualization at the corner of the contractions for different $R e_{D}^{\prime}$. Unyielded regions are displayed by vector maps for fluid $40 \mathrm{~W}$ in a), b), c), and for fluid 40W in figures d), e), and f). Red dashed-lines delimited the unyielded regions area. . . . . . . . . . . . . . . . 11

Figure 5.26-Comparison of the velocity field, lambda criterion and the curvature map for fluid 10W ( $\left.\tau_{0}^{H}=0.074 \mathrm{~Pa} ; m=0.002 \mathrm{~Pa} \cdot \mathrm{s}^{s} ; n=0.986\right)$ among the different $R e_{D}^{\prime}$ from laminar to turbulent regime. The curvature map is depicted by the red line contours, and the $\lambda_{2}$ criteria field is shown by a filled contour in gray scale. Black squares mark the position of the vortex structure center for each

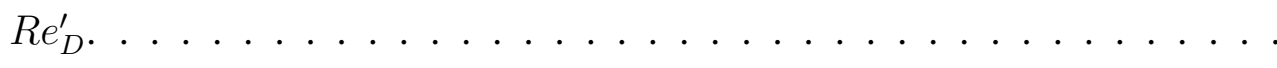

Figure 5.27-Comparison of the velocity field, lambda criterion and the curvature map for fluid 20W $\left(\tau_{0}^{H}=0.262 \mathrm{~Pa} ; m=0.251 \mathrm{~Pa} \cdot \mathrm{s}^{s} ; n=0.634\right)$ among the different $R e_{D}^{\prime}$ from laminar to turbulent regime. The curvature map is depicts by the red line contours, and the $\lambda_{2}$ criteria field is shown by a filled contour in gray scale. Black squares mark the position of the vortex structure center for each $R e_{D}^{\prime}$. . . . . . . . . . . . . . . . . . . . . 11

Figure 5.28-Displacement of the vortex core center for fluids $10 \mathrm{~W}\left(H e_{H B} \approx 0 ; n=\right.$ $0.986)$ and $20 \mathrm{~W}\left(H e_{H B}=2.938 ; n=0.634\right)$ as a function of the $R e_{D}^{\prime} \ldots 118$

Figure 5.29- $\lambda_{2}$ criteria eigenvalues along the position of the vortices center for fluid $10 \mathrm{~W}$ ( $n=0.986)$ at different $R e_{D}^{\prime}$. Dashed lines represent the radial position of the vortex center: red (upper vortex), and blue (lower vortex) . . . . . . . . . 120

Figure 5.30- $\lambda_{2}$ criteria eigenvalues along the position of the vortices center for fluid $20 \mathrm{~W}$ ( $n=0.634)$ at different $R e_{D}^{\prime}$. Dashed lines represent the radial position of the vortex center: red (upper vortex), and blue (lower vortex).

Figure 5.31-Variation of the vortex core diameter for different $R e_{D}^{\prime}$. The upper and lower vortex core are presented for fluids $10 \mathrm{~W}$ and $20 \mathrm{~W}$ 
Figure 5.32-Scheme of the effective diameter obtained by subtracting the vortex structure diameter or the major leg of the triangular unyielded region. . . . . . . . . 122

Figure 5.33-Behavior of the effective diameter ratio for the different fluids a). Reynolds number at the contraction plane, $R e_{d_{c}}^{\prime}$, in function of the $R e_{D}^{\prime} \ldots$. . . . . 123

Figure A.1 - Normalized axial velocity profile for Newtonian fluid at turbulent Reynolds numbers. . . . . . . . . . . . . . . . . . . . . 137

Figure A.2-Normalized axial velocity fluctuations, $u^{\prime+}$, as a function of the dimensionless wall distance $y^{+} \ldots \ldots \ldots$. . . . . . . . . . . . . . . . . 138

Figure A.3-Normalized radial velocity fluctuations, $v^{\prime+}$, as a function of the dimensionless wall distance $y^{+} \ldots \ldots \ldots$. . . . . . . . . . . . . . . . 139 


\section{LIST OF TABLES}

Table 3.1 - Correlations for $k_{\text {con }}$ obtained at laminar and turbulent regimes in different experimental studies for $\beta=2 \ldots \ldots \ldots$. . . . . . . . . 44

Table 4.1 - Ultrasound commercial gel properties . . . . . . . . . . . . . . . . 50

Table 4.2 - Parameters set for the different geometry configurations for each solution rheological test at HAAKE-MARS III. . . . . . . . . . . . . . . . . . . . 52

Table 4.3 - Herschel-Bulkley parameters and Hedstrom number calculated for ultrasound gel solutions. . . . . . . . . . . . . . . . . 52

Table 4.4 - Refractive properties of some materials and fluids for test regions in visualization studies. . . . . . . . . . . . . . . . . 53

Table $4.5-L e$ values required for a fully developed laminar flow at $R e_{D g}=1000$ and 1500. 56

Table 4.6 - Different tracer particles materials for fluids flow, retrieved from Raffel et al.

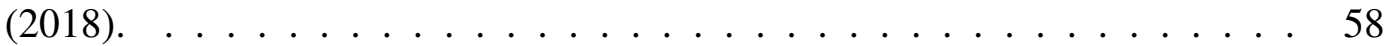

Table 4.7 - Typical range values for absorption and emission spectra of rodamine-B in water solutions. Data taken from: Zhang et al. (2014), Liu and Lu (2015). . . 60

Table 4.8 - Distance between the pressure measuring points . . . . . . . . . . . . . . . 74

Table 4.9 - Variables set to visualize fluid flow with the PIV technique . . . . . . . . 76

Table 5.1 - List of parameters set to visualize laminar fluid flow . . . . . . . . . . . 87

Table 5.2 - Listing of parameters set to visualize turbulent fluid flow . . . . . . . . . . 94

Table 5.3 - Position of the vortex structure for fluid $10 \mathrm{~W}$ at different $R e_{D}^{\prime} \ldots \ldots$. . . . 117

Table 5.4 - Position of the vortex structure for fluid $20 \mathrm{~W}$ at different $R e_{D}^{\prime} \ldots \ldots$. . . . 117

Table A.1 - Experimental parameters set for turbulent flow measurements. . . . . . . . . 136

Table B.1 - Uncertainty values of the extrapolated pressure $P_{c 1}$ and $P_{c 2}$. . . . . . . 141

Table B.2 - Uncertainty values of $k_{\text {con }}$ for the different $R e_{D}^{\prime}$ and fluids. . . . . . . . . . 143

Table B.3 - Values obtained for $M_{t}$ and $I_{t}$ as a function of the $R e_{D}^{\prime}$ for the different viscoplastic fluids. . . . . . . . . . . . . . . . . . . . . 144 


\section{LIST OF ABBREVIATIONS AND ACRONYMS}

\begin{tabular}{|c|c|}
\hline APS & Active Pixel Sensor \\
\hline $\mathrm{CC}$ & Concentric Smooth Cylinders \\
\hline $\mathrm{CCD}$ & Charged Couple Device \\
\hline CDI & Central Difference Interrogation \\
\hline CH-PP & Cross-Hatched Parallel Plates \\
\hline $\mathrm{CMC}$ & Carboxymethyl Cellulose \\
\hline CMOS & Complementary Metal-Oxyde-Semiconductor \\
\hline $\mathrm{CP}$ & Cone-Plane \\
\hline DPIV & Digital Particle Image Velocimetry \\
\hline DNS & Direct Numerical Simulation \\
\hline FDI & Forward Difference Interrogation \\
\hline GNF & Generalized Newtonian Fluid \\
\hline HGS & Hollow Glass Spheres \\
\hline IA & Interrogation Areas \\
\hline LDA & Laser Doppler Anemometer \\
\hline LDV & Laser Doppler Velocimetry \\
\hline LSV & Laser Speckle Velocimetry \\
\hline Nd:Yag & Neodymium-doped Yttrium aluminum garret \\
\hline PIV & Particle Image Velocimetry \\
\hline PMMA & Polymethyl Methacrylate \\
\hline PSP & Polyamida \\
\hline PTV & Particle Tracker Velocimetry \\
\hline PVC & Polyvinyl Chloride \\
\hline
\end{tabular}


NOMENCLATURE

\section{Roman Letters and Symbols}

A $\quad$ Area of sheared surface

$A_{T r} \quad$ Dimensionless corner unyielded region area

G Uncertainty magnitude

$h \quad$ Height of peak correlation

$N_{T} \quad$ Number of total images $\quad[-]$

$N_{X} \quad$ Number of samples [-]

$P \quad$ Pressure $\quad[P a]$

$P^{+} \quad$ Dimensionless pressure [-]

$\begin{array}{lll}P_{c 1} \quad \text { Contraction pressure at upstream region } & {[P a]}\end{array}$ 
$P_{c 2} \quad$ Contraction pressure at downstream region $\quad[P a]$

$\Delta P_{\text {con }} \quad$ Pressure drop at the contraction $[P a]$

$\Delta P_{c o n}^{\prime} \quad$ Dimensionless pressure drop at the contraction $\quad[-]$

$q \quad$ Normalized diameter of particles Mie's scattering theory

$Q \quad$ Volumetric flow rate

$\left[m^{3} s^{-1}\right]$

$Q_{N} \quad$ Volumetric flow rate for a Newtonian fluid

$\left[m^{3} s^{-1}\right]$

Radius coordinate at the plug core region

$[m]$

$r_{p}$

Pipe radius

$[m]$

$R_{p} \quad$ Plug core radius

$[m]$

Time

Reference time

Particle response time

Axial fluid velocity component

$\left[\mathrm{ms}^{-1}\right]$

Axial fluid velocity fluctuation

$\left[m s^{-1}\right]$

Normalized axial fluid velocity fluctuation

Friction velocity for Newtonian fluid

$\left[m s^{-1}\right]$

$u$

$u_{C L} \quad$ Centerline velocity

$\left[m s^{-1}\right]$

$\left[\mathrm{ms}^{-1}\right]$

$u_{x}$

Particle instantaneous velocity at $\mathrm{x}$-direction

$\left[m s^{-1}\right.$ ]

Particle instantaneous velocity at $y$-direction

$\left[m s^{-1}\right]$

$u_{z} \quad$ Velocity profile at $z$-direction

$\left[m s^{-1}\right.$ ]

Velocity of plug region at $z$-direction

$\left[m s^{-1}\right]$

Mean velocity at the test section entrance

$\left[m s^{-1}\right]$

$U_{g} \quad$ Settling velocity

$\left[\mathrm{ms}^{-1}\right]$

$U_{p} \quad$ Delay velocity

$U_{s} \quad$ Seeding particle velocity

$\left[m s^{-1}\right]$

$v \quad$ Radial fluid velocity component

$\left[m s^{-1}\right]$

$\left[m s^{-1}\right.$ ]

$v^{\prime} \quad$ Radial fluid velocity fluctuation

$\left[m s^{-1}\right]$

$v^{\prime+} \quad$ Normalized radial fluid velocity fluctuation

Volume of yielded liquid

$\left[m^{3}\right]$

$V_{c y}$

Volume of the yielded liquid

$\left[\mathrm{m}^{3}\right]$

$w \quad$ Width of peak correlation

[pixel]

$w_{x} \quad$ Standard deviation for $x$-direction

Standard deviation for $y$-direction

$X_{D} \quad$ Length of the view size in the axial direction

$y^{+} \quad$ Dimensionless wall distance

Axial direction 


\section{Functions and Tensors}

d Displacement function

H Transfer function

I Input image function

$\mathbf{I}^{\prime} \quad$ Output image function

$\hat{\mathbf{I}} \quad$ Fourier transform function of input image

$\hat{\mathbf{I}}^{\prime} \quad$ Fourier transform function of output image

$k(t) \quad$ Curvature function

N Noise function

$Q_{c} \quad$ Value for $\mathrm{Q}$ criterion

$R_{I I} \quad$ Cross-correlation function

S Deformation tensor

tr Transpose

$f(x, y) \quad$ Interpolation function

$\Omega \quad$ Rotation tensor

\section{Greek Letters}

$\begin{array}{ll}\Delta y & \text { Particles displacement in y-direction } \\ \varepsilon & \text { Rate of dissipation of turbulent kinetic energy }\end{array}$

$\left[m^{2} \cdot s^{-3}\right]$

$\eta \quad$ Viscoplastic fluid apparent viscosity

$\left[P a \cdot s^{n}\right]$

$\eta_{k} \quad$ Kolmogorov length scale

$[m]$

$\eta_{0} \quad$ Viscoplastic fluid apparent viscosity for low shear rates

$[P a \cdot s]$

$\Theta \quad$ Magnification factor of the camera

$\kappa \quad$ Flow curvature

$\lambda \quad$ Wavelength

$[\mathrm{nm}]$

$\lambda_{s} \quad$ Relaxation time

$[s]$

$\lambda_{2} \quad$ Second eigenvalue for Lambda criterion

$\mu \quad$ Newtonian fluid dynamic apparent viscosity

$\mu_{0}^{B} \quad$ Plastic viscosity for Bingham model

$[P a \cdot s]$ 


$\begin{array}{llc}\mu_{C} & \text { Apparent viscosity for Casson model } & {[\mathrm{Pa} \cdot \mathrm{s}]} \\ \mu_{w} & \text { Mean wall viscosity } & {[\mathrm{Pa} \cdot \mathrm{s}]} \\ \nu & \text { Kinematic viscosity } & {\left[\mathrm{m}^{2} \cdot \mathrm{s}^{-1}\right]} \\ \rho & \text { Density } & {\left[\mathrm{kg} \cdot \mathrm{m}^{-3}\right]} \\ \rho_{p} & \text { Particle density } & {\left[\mathrm{kg} \cdot \mathrm{m}^{-3}\right]} \\ \tau_{y x} & \text { Shear stress at } x y \text {-coordinates } & {[\mathrm{Pa}]} \\ \sigma_{X} & \text { Standard deviation } & {[-]} \\ \sigma_{i i} & \text { Deformation stress Von Misses Criteria } & {[\mathrm{Pa}]} \\ \tau & \text { Shear stress } & {[\mathrm{Pa}]} \\ \tau_{0} & \text { Yield stress } & {[\mathrm{Pa}]} \\ \tau_{0}^{B} & \text { Yield stress for Bingham model } & {[\mathrm{Pa}]} \\ \tau_{0}^{H} & \text { Yield stress for Herschel-Bulkley model } & {[\mathrm{Pa}]} \\ \tau_{0}^{C} & \text { Yield stress for Casson model } & {[\mathrm{Pa}]} \\ \tau_{r z} & \text { Shear stress at } r z \text {-coordinates } & {[\mathrm{Pa}]} \\ \tau \gamma_{y x} & \text { Shear stress at } x y \text {-coordinates } & {[\mathrm{Pa}]} \\ \tau_{k} & \text { Kolmogorov time scale } & {[\mathrm{s}]} \\ \tau_{w} & \text { Wall shear stress magnitude } & {[\mathrm{Pa}]} \\ \tau^{*} & \text { Dimensionless shear stress } & {[-]} \\ \phi & \text { Shear stress ratio } & {[-]} \\ \Phi & \text { Displacement efficiency } & {[-]} \\ \Omega & \text { Dimensionless flow rate } & {[-]}\end{array}$

\section{Non-dimensional Parameters}

$\mathrm{Bi}$ Bingham number [-]

$H B \quad$ Herschel-Bulkley number [-]

He Hedstrom number [-]

$\mathrm{He}_{B} \quad$ Hedstrom number for Bingham model [-]

$H e_{H B} \quad$ Hedstrom number for Herschel-Bulkley model [-]

Re Reynolds number [-]

$R e_{D g} \quad$ Generalized Reynolds number at the test section entrance [-]

$R e_{g} \quad$ Generalized Reynolds number [-]

$R e_{b c} \quad$ Critical Reynolds number Hanks criteria [-]

$R_{B} \quad$ Reynolds number for Bingham model [-]

$R e_{d_{c}} \quad$ Reynolds number at the entrance of the contraction [-]

$R e_{\text {gen } H B} \quad$ Reynolds number for generalized Herschel-Bulkley fluids [-]

$R e_{\text {mod }} \quad$ Modified Reynolds number for annular flow [-]

$R e_{M R} \quad$ Reynolds number - Metzner and Reeds [-] 
$R e^{\prime} \quad$ Reynolds number Malin criteria

\section{Superscripts and Subscripts}
( ) $1 \quad$ Evaluated at the upstream region
( ) $2 \quad$ Evaluated at the downstream region
()$_{D} \quad$ Evaluated at the contraction plane
()$_{D} \quad$ Evaluated at the test section entrance
()$^{T} \quad$ Transpose
()$_{w} \quad$ Evaluated at the wall
()$_{v x_{u p}} \quad$ Evaluated at the upper vortex core
( ) $v x_{d n} \quad$ Evaluated at the lower vortex core

\section{Operators}

$d() / d() \quad$ Gradient

$\delta($ ) Uncertainty value for a variable

$\partial() / \partial() \quad$ Coefficient of sensitivity for a variable

$\partial_{t}() \quad$ Partial time derivate 


\section{CONTENTS}

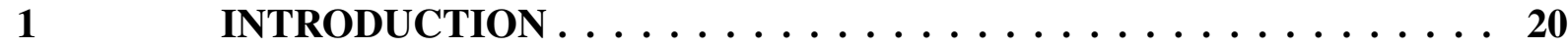

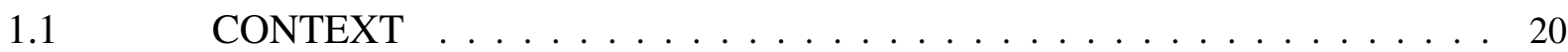

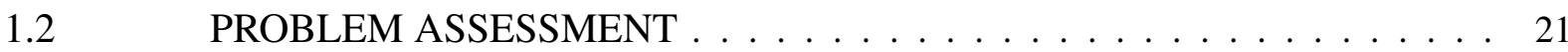

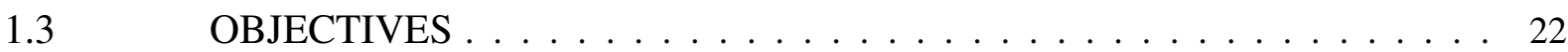

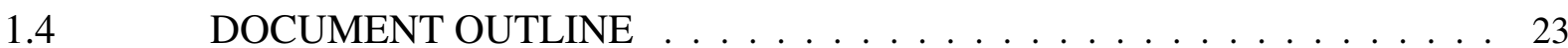

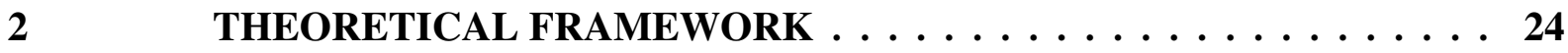

$2.1 \quad$ FLUID BEHAVIOR $\ldots \ldots \ldots \ldots \ldots \ldots \ldots \ldots \ldots \ldots$

$2.1 .1 \quad$ Newtonian Fluid $\ldots \ldots \ldots \ldots \ldots \ldots \ldots$

2.1.2 Non-Newtonian Fluid . . . . . . . . . . . . . . . . . . . . . . . . . 25

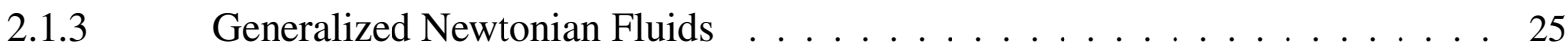

2.1.3.1 Shear-thinning fluids . . . . . . . . . . . . . . . . . . . 26

2.1.3.2 Shear-thickening fluids . . . . . . . . . . . . . . . . . . 26

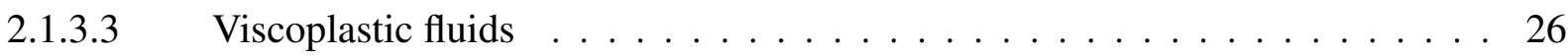

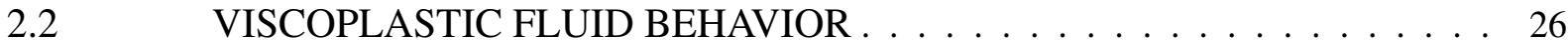

2.2.1 Mathematical Models for Viscoplastic Behavior . . . . . . . . . . . 27

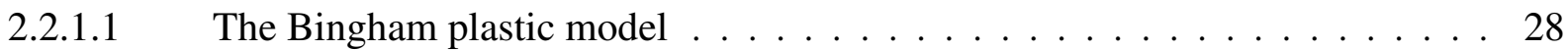

2.2.1.2 The Herschel-Bulkley fluid model . . . . . . . . . . . . . . . . 28

2.2.1.3 The Casson fluid model . . . . . . . . . . . . . . . . . . . . . 28

2.2.2 Laminar Flow in Circular Pipes . . . . . . . . . . . . . . . . . . . . . . 29

2.2.3 Friction Factors in Transitional and Turbulent Flow Conditions . . . . . . . 34

2.2.4 Flow Structure of Viscoplastic Flow in Contractions . . . . . . . . . . . . . 35

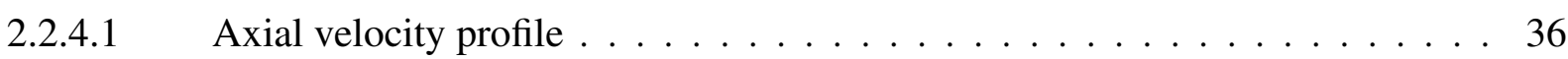

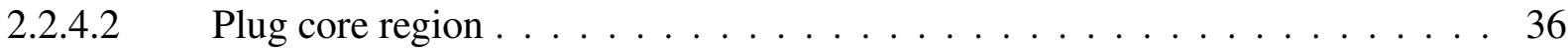

2.2.4.3 Unyielded corner regions and vortex structures . . . . . . . . . . . . 37

2.2.4.4 Aspect ratio . . . . . . . . . . . . . . . . . . . . . . . . . 37

2.2.4.5 Pressure loss coefficient . . . . . . . . . . . . . . . 37

2.3 FUNDAMENTAL PRINCIPLES OF PARTICLE IMAGE VELOCIMETRY

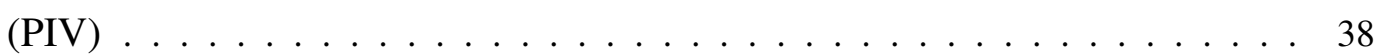

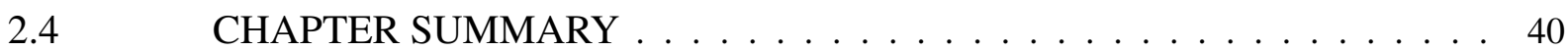

3 LITERATURE REVIEW .................41

3.1 FLOW OF VISCOPLASTIC FLUIDS IN STRAIGHT TUBES $\ldots \ldots \ldots$.

3.2 FLOW OF VISCOPLASTIC FLUIDS IN CONTRACTIONS . . . . . . . 42

3.3 VISUALIZATION OF VISCOPLASTIC FLUIDS FLOW IN CONTRACTIONS BY PIV . . . . . . . . . . . . . . . . . . . . . 45

3.4 CRITERIA FOR VISUALIZATION OF FLOWS BY PIV TECHNIQUE . . . 46

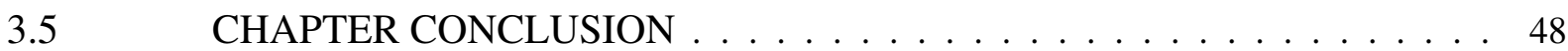

4 METHODOLOGY $\ldots \ldots \ldots \ldots \ldots \ldots \ldots \ldots \ldots \ldots \ldots$

$4.1 \quad$ DESIGN PARAMETERS $\ldots \ldots \ldots \ldots \ldots$ 
$4.1 .1 \quad$ Working Fluid $\ldots \ldots \ldots \ldots \ldots \ldots$

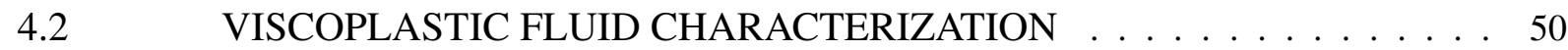

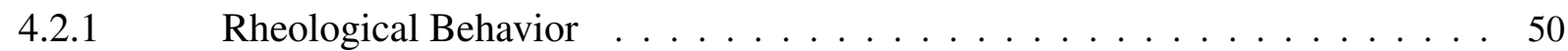

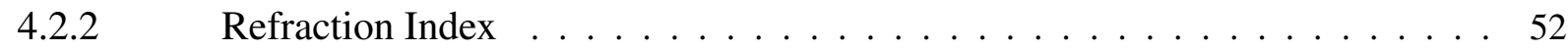

$4.3 \quad$ EXPERIMENTAL SETUP $\ldots \ldots \ldots \ldots \ldots \ldots \ldots$

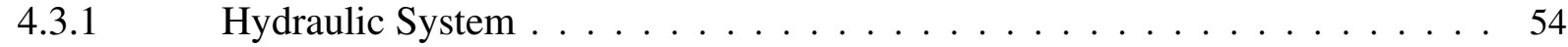

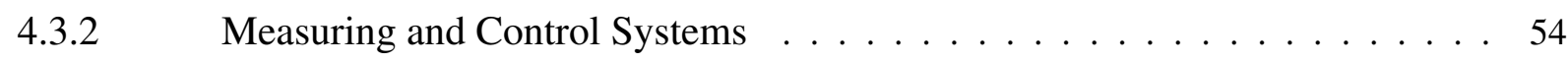

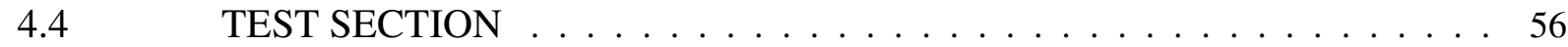

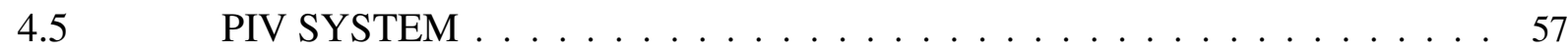

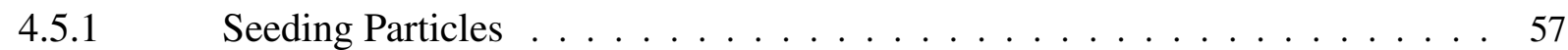

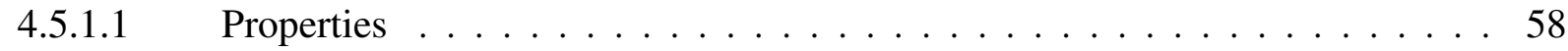

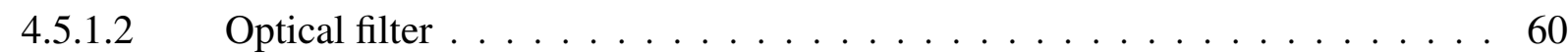

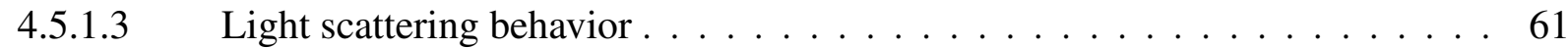

$4.5 .2 \quad$ Image Recording . . . . . . . . . . . . . . . . 62

4.5.3 Method for Image Acquisition $\ldots \ldots \ldots \ldots \ldots$

4.5.4 Image Correlation . . . . . . . . . . . . . . . . . . . 64

4.5.5 Peak Detection and Displacement Estimation . . . . . . . . . . . . . 66

4.5.6 Adaptive Interrogation Technique . . . . . . . . . . . . . . . 68

4.5.6.1 Overlap method . . . . . . . . . . . . . . . . . . . . . . 69

4.5.6.2 Multiple pass interrogation and offset method . . . . . . . . . . . . 69

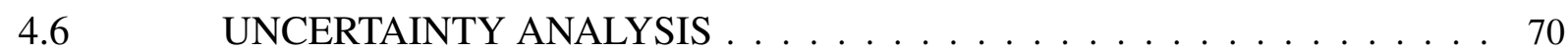

4.6.1 Uncertainty Analysis for Measurement Devices . . . . . . . . . . . . . 71

4.6.2 Uncertainty Analysis for PIV Technique . . . . . . . . . . . . . 71

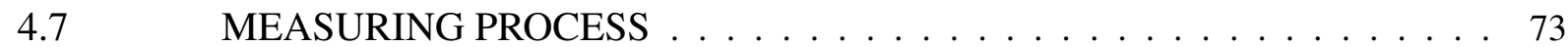

4.7.1 Pressure Measurements and Pressure Loss Coefficient Calculation . . . . . . . 73

4.7.2 Vectors, Velocity Fields and Derived Quantities ～. . . . . . . . . . . . 74

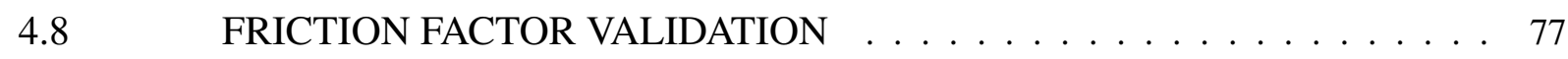

$5 \quad$ RESULTS AND DISCUSSIONS $\ldots \ldots \ldots \ldots \ldots \ldots \ldots$

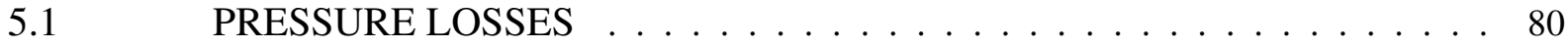

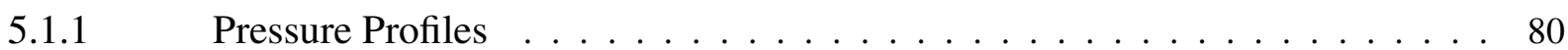

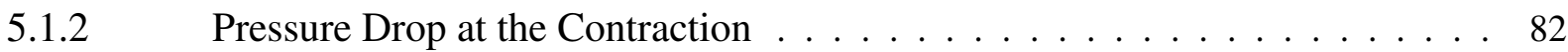

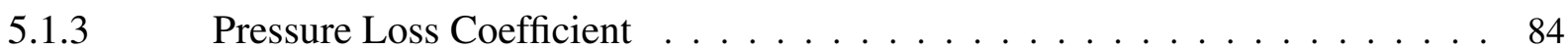

$5.2 \quad$ FLOW FIELD AND MEAN QUANTITIES $\ldots \ldots \ldots \ldots$

$5.2 .1 \quad$ Mean Velocity Vector Map $\ldots \ldots \ldots \ldots \ldots$

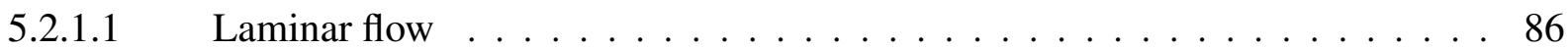

$5.2 .1 .2 \quad$ Turbulent flow . . . . . . . . . . . . . . . . . 91

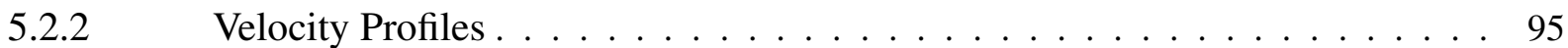

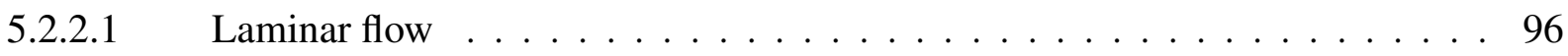

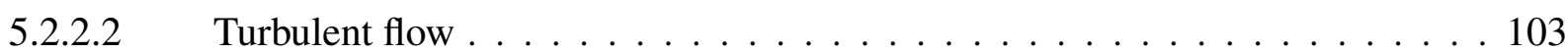

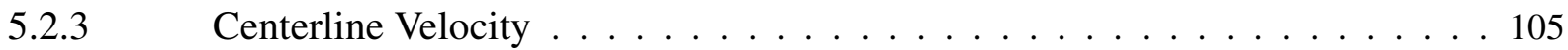

5.2 .4 Turbulent Statistics $\ldots \ldots \ldots \ldots \ldots \ldots$

$5.3 \quad$ VORTEX STRUCTURES AND UNYIELDED REGIONS . . . . . . . . . 110 
5.3.1 Visualization of the Contraction Corners . . . . . . . . . . . . . . 110

5.3.2 Identification of Vortex Core and Vortex Core Center . . . . . . . . . . . 113

5.3.2.1 Lambda criterion . . . . . . . . . . . . . . . . . . . . . . . . 114

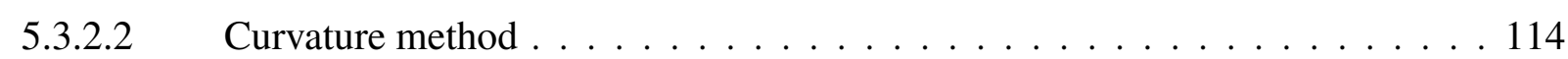

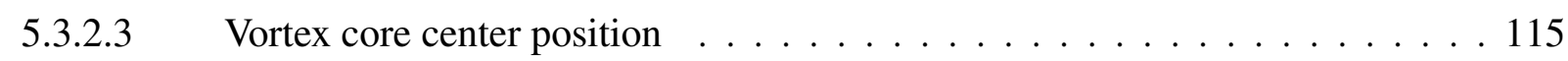

5.3.2.4 Vortex core area . . . . . . . . . . . . . . . . . . . . 119

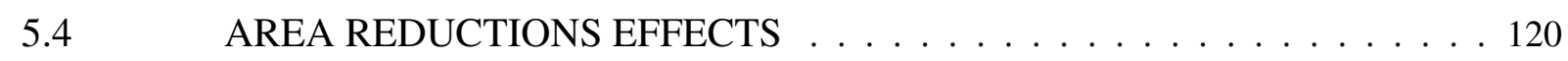

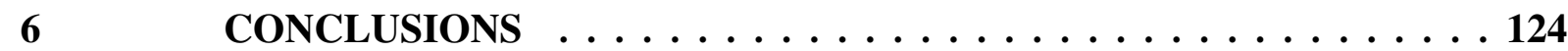

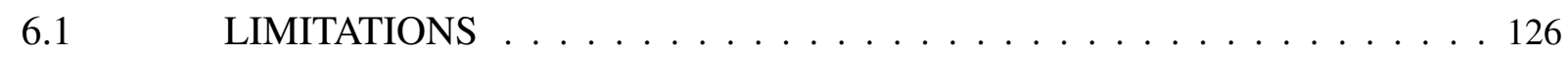

6.2 SUGGESTIONS FOR FUTURE RESEARCH . . . . . . . . . . . . . 126

BIBLIOGRAPHY . . . . . . . . . . . . . . . 128

APPENDIX A - PIV CALIBRATION TESTS . . . . . . . . 135

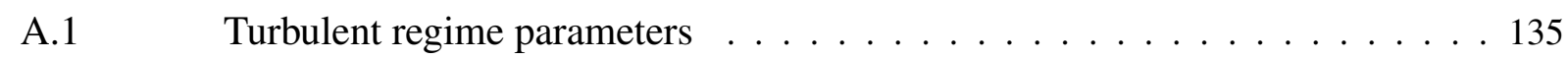

APPENDIX B - UNCERTAINTY ANALYSIS . . . . . . . . . . . 140

B.1 Pressure measurements and derivate quantities uncertainties . . . . . . . . 140

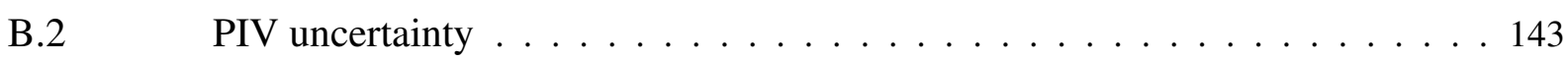




\section{INTRODUCTION}

\subsection{CONTEXT}

The flow of non-Newtonian materials through abrupt contractions is commonly encountered in natural and industrial processes such as polymer mold filling, oil well drilling, heat exchangers, among others. In the oil industry, non-Newtonian materials such as slurries, xanthan gum, sucrose, and polymer solutions are commonly used, some of them as drilling fluids (KFURI et al., 2011). The drilling fluid used during the drilling of oil wells flows into a drill string and inner channels, which implies the passage through sudden contractions. This results in pressure and energy losses (FESTER et al., 2008), the appearance of secondary flow regions such as vortex structures. Figure 1.1 shows an schematic representation' for the flow of the drilling fluid into a drilling string.

Figure 1.1 - Basic parts and contractions of a drilling string. Contractions are indicated by red arrows.

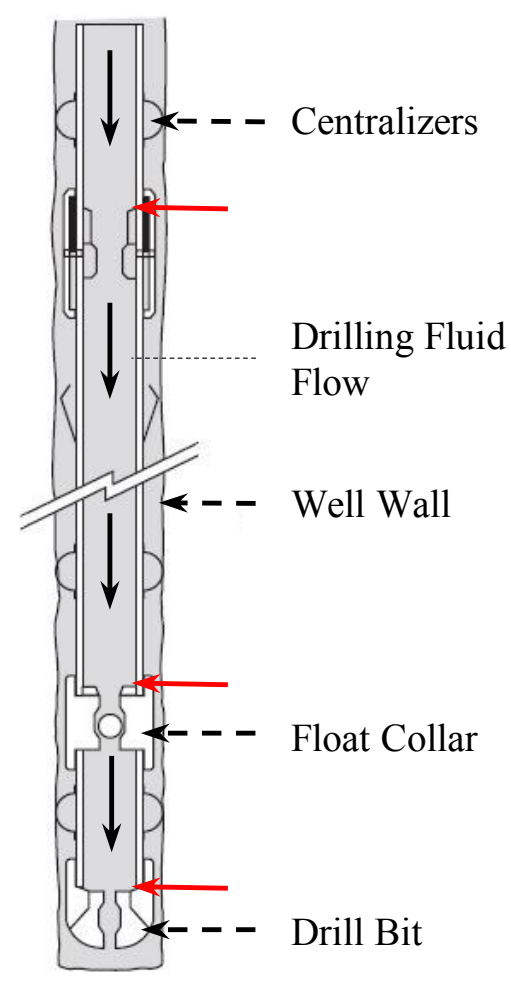

Source: Retrieved from http://www.drillingcourse.com/2015/12/cementing-jobs.

These drilling fluids display complex characteristics such as elastoviscoplasticity and thixotropy, which means reversible time-dependent decreasing viscosity under shear and display yield stress that characterizes the transition from a gelled fluid to an yielded one induced by shearing (COUSSOT et al., 2002; BALMFORTH et al., 2014; BONN et al., 2017). For example, when the flow through the drill string is interrupted, the drilling fluid forms a gelled structure to maintain the rock cuttings suspended due to viscoplastic an thixotropic properties presented in these fluids.

In order to understand the behavior of the non-Newtonian fluids, their rheological properties can be 
studied separately. Then, it is possible to isolate their viscoplastic behavior that can be well represented by water-based solutions with biopolymers (HAMED; BELHADRI, 2009). Such as Carbopol neutralized gels, that have been used in numerous applications as viscoplastic fluid over the past years (PIAU, 2007), due to their low thixotropy, microstructural stability, optical transparency, and stable rheological properties (CURRAN et al., 2002; PIAU, 2007; OVARLEZ et al., 2013).

\subsection{PROBLEM ASSESSMENT}

As mentioned in section 1.1, the viscoplastic fluid flow through abrupt contractions results in pressure losses, the appearance of vortex structures and secondary flow regions at the upstream contraction zone as detailed in Fig.1.2.

Figure 1.2 - Geometry of the flow through an abrupt circular contraction.

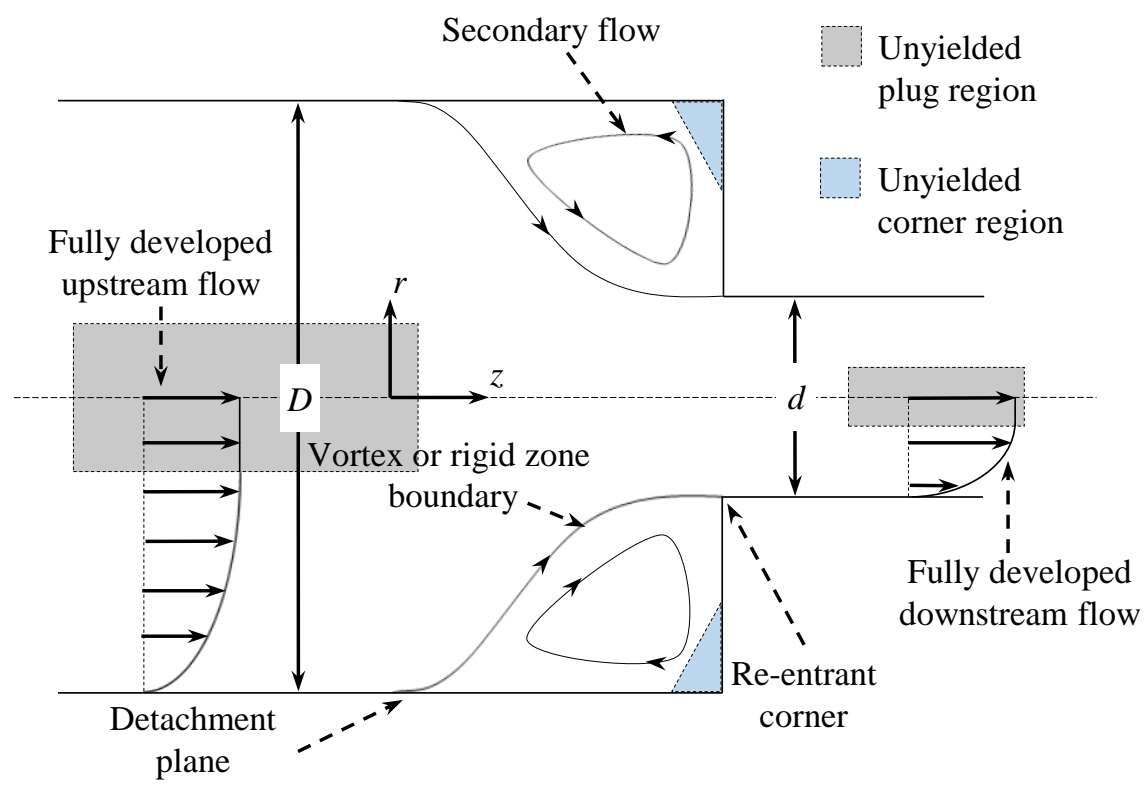

Source: Adapted from Boger et al. (1992).

The fluid flows from a pipe of diameter $D$ to a smaller diameter pipe $d$, these variables define the contraction ratio by $\beta=D / d$. When fully developed laminar flow is reached, peripheral viscous flow zones and unyielded regions appear. In the contraction corners, it is possible to have vortex structures, sometimes with the presence of unyielded regions. In these unyielded regions, the fluid does not flow as the shear stress is lower than the fluid yield stress (JAY et al., 2002). As the flow approaches the contraction, a detachment of the flow from the wall is generated due to the high shear rates, pressure drop, and the increase of the flow velocity. Then, at the re-entrant corner, the shear rate decreases and unyielded regions reappear at the upstream zone of the contraction.

This particular behavior motivated the Research Center for Rheology and Non-Newtonian Fluids $(C E R N N)$ to carry out numerical and experimental studies with abrupt contractions focused on the dynamic of the viscoplastic fluid flow. As carbopol-water solutions exhibit the plastic behavior of drilling fluids, 
it has been suggested as a model to study the viscoplastic behavior of drilling fluids in experimental researches.

Although flow through abrupt contractions are common in practical applications, there are few projects and studies focused on the experimental study of the dynamics of this type of flow (PALACIOS, 2011). For example, numerical and experimental studies, and works on viscoplastic fluids are far less numerous than those on viscoelastic ones (JAY et al., 2002). Some authors, as ??) and Sisavath et al. (2002) indicated that several studies were done just on laminar flow of non-Newtonian fluids through sudden contractions, but not in the turbulent regime. Also, most experimental studies for non-Newtonian fluids did not agree with one another or with analytical and numerical solutions (FESTER et al., 2008).

Due to the lack of quantitative knowledge of the viscoplastic fluid flow behavior through area reduction, it is necessary to use non-intrusive techniques for flow visualization and measurement, such as Particle Image Velocimetry (PIV). This is a powerful tool to analyze flow details, especially in non-trivial geometries, such as planar and axisymmetric abrupt contractions for non-Newtonian fluids. From a technological point of view, for example, to design some industrial process, it is very important to know the structure of the flow and how it changes in accordance with the governing parameters, such as the yield stress, shear rate, contraction ratio, and Reynolds number. In some industrial cases, such as in the oil well drilling, it is necessary to predict the size of unyielded zones and the size of the vortex structures, and also controlling the pressure losses generated by the sudden passage of the flow through area reductions.

\subsection{OBJECTIVES}

The objective of the present study is to visualize and analyze the flow structure before the contraction for different Reynolds and Hedström numbers, as well as their effect on the velocity profiles, generation of vortex structures, the variation of unyielded regions area and the behavior of pressure drop at the contraction, in order to contribute to the understanding of the viscoplastic flow behavior.

To reach this objective are accomplished the specific aims:

- Design and use an experimental flow-loop, and equipped with a PIV system and instruments for monitoring the variation of mass flow rate, pressures losses, temperature, and density during the viscoplastic fluid flow.

- Visualize the behavior of the structure for different Carbopol solutions flow, for laminar and turbulent regimes with Reynolds numbers $(R e)$ between 500 and 1500 for laminar conditions and $R e$ between 2000 and 10000 for the turbulent ones. The concentrations of carbopol are set by Hedström numbers $\left(H e_{H B}\right)$ between 1 and 20.

- Analyze the vector fields, turbulent fluctuations and vortex structures visualized through the PIV technique in order to correlate the dependence of the flow structure with the variation of the Hedström and the Reynolds numbers for a viscoplastic fluid flow. As well as the dependence of the pressure drop coefficient at the contraction with the variation of these dimensionless parameters. 


\subsection{DOCUMENT OUTLINE}

The content of this work is structured in 6 chapters; the present chapter presents the introduction to the problem under study and the objectives accomplished.

In chapter 2, basic concepts about flow of non-Newtonian fluids, and definitions related to the flow of this fluids in axisymmetric contractions are described. Also, the main theoretical aspects of the PIV technique, as some relevant parts of the experimental setup, particles, parameters, formation, correlation of images, and mathematical expressions that describe some PIV configuration.

Chapter 3 presents a literature review for this type of flow, showing relevant previous experimental and numerical studies related to the flow in abrupt contractions of non-Newtonian fluids.

The proposed experimental approach related to the design of the experimental bench, the PIV system in the testing section, the construction of the contraction prototype, the characterization of the working fluids and the analysis of uncertainties in the experimental tests are described in Chapter 4. Furthermore, the initial calibration of the system was performed, with the study of the flow of Newtonian fluids in a circular section tube for laminar and turbulent regimes.

Chapter 5 discusses and compares the results obtained with the data available in the literature. First, the pressure drop along the flow-loop is studied as a function of the Reynolds number and the rheological properties for each viscoplastic fluid. Also, the pressure loss coefficient at the contraction is evaluated and compared with other author correlations. The experimental results obtained by the PIV technique as the mean velocity vector field, velocity fluctuations, centerline behavior, and the vortex restructures as the unyielded regions at the corner of the contraction are presented, discussed and related with the Reynolds and the Hedström number.

In chapter 6 the main conclusions from this project are summarized and the purposes and the expectations of this experimental study are emphasized.

Finally, the appendices show the results obtained from PIV technique validation tests with Newtonian fluids at different Reynold number in the turbulent regime. The data are compared with other techniques to evaluate the accuracy of the methodology implemented. Moreover, the error analysis involved in the developing of this experimental study is shown. 


\section{THEORETICAL FRAMEWORK}

This chapter introduces some fundamental concepts regarding Newtonian and non-Newtonian fluids, their response to externally applied pressure and the effects produced under the action of a shear rate and the flow through circular tubes configurations. Also, some principles of the Particle Image Velocimetry (PIV) technique are presented.

\subsection{FLUID BEHAVIOR}

The following section described basic concepts about the behavior of the types fluids. This section is focused on the generalized Newtonian Fluids and the models used to describe their behavior.

\subsubsection{Newtonian Fluid}

Considering a thin layer of a fluid contained between two parallel plates at a short distance $(d y)$, and supposing now that one surface is moving with a constant velocity $(d U)$, while the other remains at rest as shown in Fig. 2.1. For a Newtonian fluid the relationship between the shear stress and the velocity gradient is a constant known as dynamic viscosity or just viscosity $(\mu)$, that is not dependent of the shear stress $(\tau)$ and the velocity gradient, being only a function of the material, at some specific temperature and pressure (CHHABRA; RICHARDSON, 2011).

Figure 2.1 - Representation of unidirectional shearing flow.

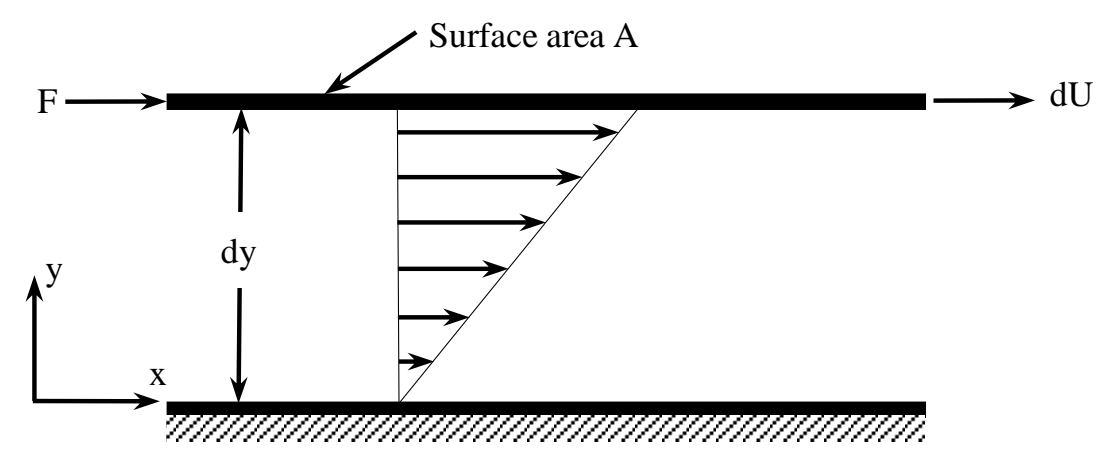

Source: Chhabra and Richardson (2011).

In this simple case, the shear rate $(\dot{\gamma})$ may be expressed as the velocity gradient in the direction perpendicular to the shear force $(F)$, and the shear stress can be expressed as:

$$
\frac{F}{A}=\tau_{y x}=\mu\left(-\frac{d U}{d y}\right)=\mu \dot{\gamma}_{y x}
$$

where the first subscript in both $\tau$ and $\dot{\gamma}$ indicates the direction normal to the shearing surface, while the second subscript refers to the direction of the force and the flow. 
It can be seen that at any shear plane there are two equal and opposite shear stresses, a positive one on the slower moving fluid and a negative one on the faster moving fluid layer, a cause-effect reaction between the layers. The negative sign in equation (2.1) means that $\tau_{y x}$ is a resistance to motion. The equation could be expressed for a incompressible fluid with density $\rho$, as:

$$
\tau_{y x}=-\frac{\mu}{\rho} \frac{d}{d y}(\rho U)
$$

The quantity $\rho U$ is the linear momentum in the $x$-direction per unit volume of the fluid, hence $\tau_{y x}$ represents the momentum flux in the $y$-direction and the negative sign indicates that the momentum transfer occurs in the direction of decreasing velocity.

The viscosity of the fluid can be understood as the resistance to a gradual deformation by the shear stress sensed for an infinitesimal fluid layer. Barnes (2000) argued that a substance is classified as Newtonian fluid if the dynamic viscosity giving by Eq. (2.1), it is independent of both strain rate and time, at a given temperature and pressure. A Newtonian fluid must not display any elastic or extensional properties. Therefore, the plot $\tau$ vs $\dot{\gamma}$ will be a straight line passing through the origin of coordinates. Despite the simplicity of Newton's law, a large number of fluids can be represented by the equation (2.1). According to Chhabra and Richardson (2011), gases, simple organic liquids and molten metals exhibit a Newtonian behavior.

\subsubsection{Non-Newtonian Fluid}

In the case of a non-Newtonian fluid, its flow curve is nonlinear or does not pass through the origin, and also can exhibit some particular characteristics as elasticity. Moreover, the viscosity varies according to the shear stress imposed over the material, defining a new concept known as apparent viscosity $(\eta)$, which is the ratio between the shear stress and the shear rate (CHHABRA; RICHARDSON, 2011). In some conditions, the apparent viscosity is also dependent on the kinematic history of the fluid. Such materials may be conveniently grouped into three general classes (DESHPANDE, 2010a):

- Fluids for which the shear stress is determined only by the shear rate; these fluids are known as: time independent, purely viscous, inelastic or generalized Newtonian fluids (GNFs).

- More complex fluids for which the apparent viscosity depends on the duration of shearing and their kinematic history, these fluids are called time-dependent.

- Materials exhibiting characteristics of both: fluids and elastic solids; and also show a partial relaxation and an elastic recovery. These type of materials are categorized as viscoelastic fluids.

For a better understanding, the Generalized Newtonian Fluids are described in the following subsection.

\subsubsection{Generalized Newtonian Fluids}

These fluids are characterized by the dependence of the shear rate on the shear stress only (CHHABRA; RICHARDSON, 2011; DESHPANDE, 2010a). Depending on the mathematical form of this relationship, most distinguished fluids in this category are: 
- Shear-thinning or pseudoplastic fluids

- Shear-thickening or dilatant fluids

- Viscoplastic fluids

\subsubsection{Shear-thinning fluids}

This non-Newtonian fluid behavior is characterized by an apparent viscosity $\eta$, which decreases with increasing shear rate, as shown in figure 2.2. Many mathematical models for describing shear-thinning fluid behavior can be found in the literature, some of these are: power law or Ostwald de Waele model, the Carreau model, and the Cross model (CHHABRA; RICHARDSON, 2011; BIRD et al., 1987). According to Fall et al. (2008), shear-thinning fluids are the most widely encountered type of non-Newtonian time-independent behavior. Most colloids, polymers, and proteins in a solvent exhibit pseudo-plastic characteristics.

\subsubsection{Shear-thickening fluids}

For some concentrated suspensions of particles, shear thickening behavior can be observed as an abrupt increase of the apparent viscosity $\eta$ with increasing shear rate (BARNES et al., 1989; BOERSMA et al., 1990; FALL et al., 2010; KHANDAVALLI; ROTHSTEIN, 2015; NA et al., 2016). Under the action of an external stress or shear, the shear-thickening fluid becomes a yield material and its physical state transform to a solid-like state (HE et al., 2015). This process is reversible, the fluid-like state will return once the applied stress is removed. The shear-thickening fluids can be represented, in general, by power law mathematical models (FRITZ et al., 2002).

\subsubsection{Viscoplastic fluids}

A viscoplastic material does not deform until the applied shear stress exceeds a critical value, this threshold is known as the yield stress, $\tau_{0}$. Such material behaves like a solid for shear stress values below the yield stress, and like a viscous fluid for stress values above the yield stress (MEWIS; SPAULL, 1976; DZUY; BOGER, 1983; BIRD et al., 1983; BARNES; WALTERS, 1985; CHENG, 1986; KEE; DURNING, 1990; MØLLER et al., 2006; MØLLER et al., 2009; COUSSOT et al., 2017), as displayed in figure 2.2. Materials used in cosmetic and industrial applications such as mayonnaise, shaving foams and toothpaste exhibit a viscoplastic behavior. These materials are well represented by some models, like the Bingham and the Herschel-Bulkley model.

Viscoplastic fluids behavior is detailed in the next section in this chapter, also the mathematical models that represent this type of material.

\subsection{VISCOPLASTIC FLUID BEHAVIOR}

As previously explained, this type of fluid is characterized by the existence of a yield stress $\tau_{0}$, which must be exceeded for the fluid to flow (CHHABRA; RICHARDSON, 2011). This kind of behavior can 
Figure 2.2 - Flow curves schema for Newtonian and non-Newtonian fluids.

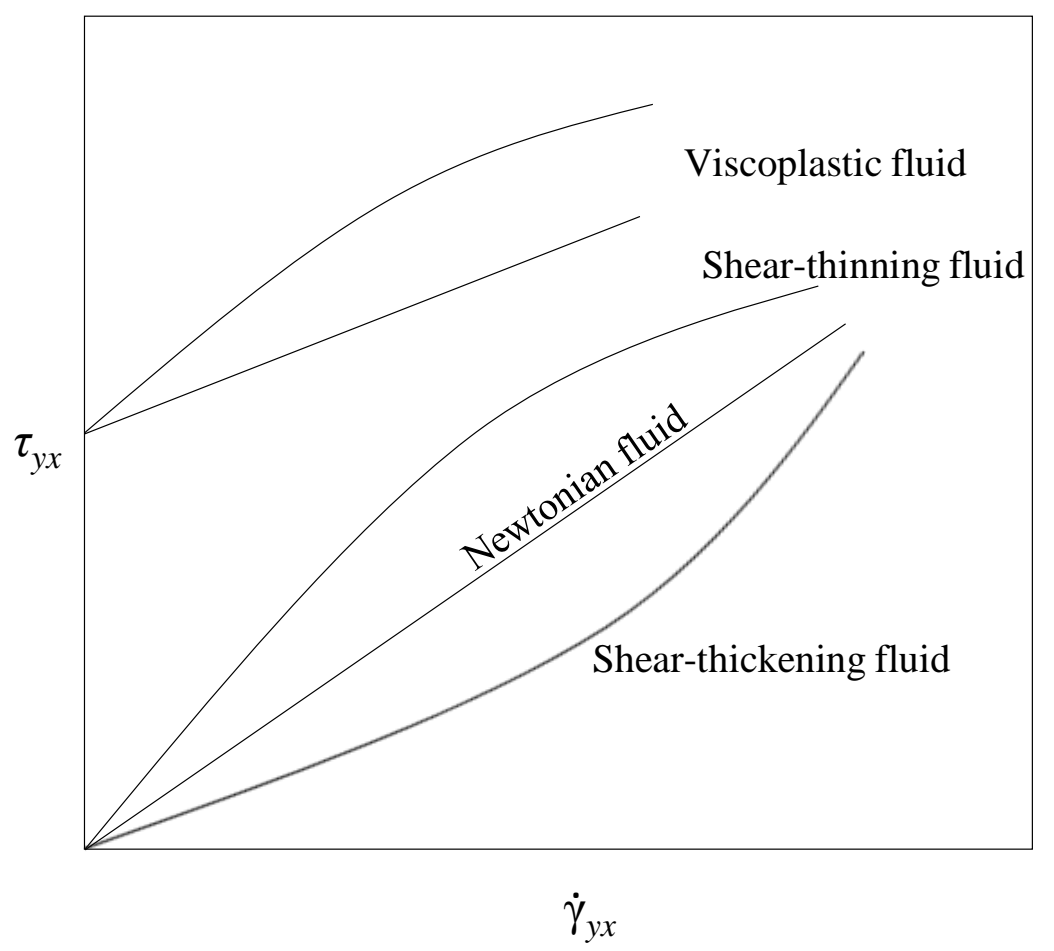

Source: Adapted from Bird et al. (1987).

be explained if the substance at rest is considered as a group of rigid three-dimensional structures, that resist any external stress smaller than $\tau_{0}$. For stress larger than $\tau_{0}$, the structure breaks down, and the material behaves like a viscous material. In some cases, the build-up and breakdown of the structure have been found to be reversible, i.e., the substance may regain its initial value of yield stress (CHHABRA; RICHARDSON, 2011).

The viscoplastic materials display an apparent viscosity, which decreases with shear rate increases. For low shear rates, the apparent viscosity is considered as infinite at the instant before the substance yields and begins to flow. It is thus possible to regard these materials as having a particular class of shear-thinning behavior. It is virtually impossible to ascertain whether any real material has yield stress or not, but nevertheless, the concept of the yield stress has been convenient in practice due to some materials approximate very close to this type of behavior (CHHABRA; RICHARDSON, 2011). The advent of constant-stress rheometers has made it possible to scrutinize the behavior of materials at very low shear rates. Common examples of viscoplastic fluid behavior include some particulate suspensions, emulsions, foodstuffs, blood, gels, drilling fluids, and muds (BARNES, 1999).

\subsubsection{Mathematical Models for Viscoplastic Behavior}

Many empirical expressions have been proposed as a result of curve-fitting examinations. There are three common models to describe viscoplastic fluids, described in the following sections. The most common models used for viscoplastic fluid are the Bingham, Casson and the Herschel-Bulkley model. These rheological models describe the shear-thinning behavior of the fluids and the presence of yield 
stress.

\subsubsection{The Bingham plastic model}

It is a simple equation to describe the behavior of a fluid with a yield stress. For one-dimensional shear, it is written as (CHHABRA; RICHARDSON, 2011; MORRISON, 2001):

$$
\begin{array}{lll}
\tau_{y x}=\tau_{0}^{B}+\eta_{B}\left(\dot{\gamma}_{y x}\right) & \text { for } & \left|\tau_{y x}\right|>\tau_{0}^{B} \\
\dot{\gamma}_{y x}=0 & \text { for } & \left|\tau_{y x}\right|<\tau_{0}^{B}
\end{array}
$$

The model also can be written as Hookean behavior at stresses below yielding.

$$
\begin{array}{lll}
\tau_{y x}=\tau_{0}^{B}+\eta_{B}\left(\dot{\gamma}_{y x}\right) & \text { for } & \left|\tau_{y x}\right|>\tau_{0}^{B} \\
\tau_{y x}=K \gamma_{y x} & \text { for } & \left|\tau_{y x}\right|<\tau_{0}^{B}
\end{array}
$$

The parameters $\tau_{0}^{B}$ and $\eta_{B}$ are curve-fitting constants, the yield stress and the apparent viscosity fit for this fluid model, and $K$ is the Young's modulus.

\subsubsection{The Herschel-Bulkley fluid model}

It is a generalization of the Bingham model to consider the non-linear flow curves for $\left|\tau_{y x}\right|>\tau_{0}^{B}$. In one-dimensional steady shearing motion, the model is given by (CHHABRA; RICHARDSON, 2011):

$$
\begin{array}{lll}
\tau_{y x}=\tau_{0}^{H}+m\left(\dot{\gamma}_{y x}\right)^{n} & \text { for } & \left|\tau_{y x}\right|>\tau_{0}^{H} \\
\dot{\gamma}_{y x}=0 & \text { for } & \left|\tau_{y x}\right|<\tau_{0}^{H}
\end{array}
$$

In this model, $m$ and $n$ are two empirical curve-fitting parameters and are know as the fluid consistency coefficient and the flow behavior index, respectively. When the flow behavior index $(n)$ reaches values below the unity, the viscoplastic material exhibits shear-thinning properties. With the use of three constant parameters, the Herschel-Bulkley provides a better fit to some experimental data.

\subsubsection{The Casson fluid model}

This model has often been used for describing the steady shear stress and shear rate behavior of some fluids such as blood, molten chocolates and also some particles suspensions that have a behavior closely described by this model, where $\eta_{C}$ is the plastic viscosity determined for this model (CHHABRA; RICHARDSON, 2011; MALKIN, 1994),

$$
\begin{array}{ll}
\left(\left|\tau_{y x}\right|\right)^{1 / 2}=\left(\tau_{0}^{C}\right)^{1 / 2}+\left(\eta_{C}\left|\dot{\gamma}_{y x}\right|\right)^{1 / 2} & \text { for }\left|\tau_{y x}\right|>\tau_{0}^{C} \\
\dot{\gamma}_{y x}=0 & \text { for }\left|\tau_{y x}\right|<\tau_{0}^{C}
\end{array}
$$

The three viscoplastic fluid models are sketched in figure 2.3. At each model $\tau_{y x}$ is represented as a function of the shear rate. There are other empirical models containing a yield stress, such as the generalized Casson equation, the Crowley-Kitzes model, the Shul'man equation, among others (BIRD et al., 1983). 
Figure 2.3 - Sketch of empirical curve-fitting models for viscoplastic fluids.

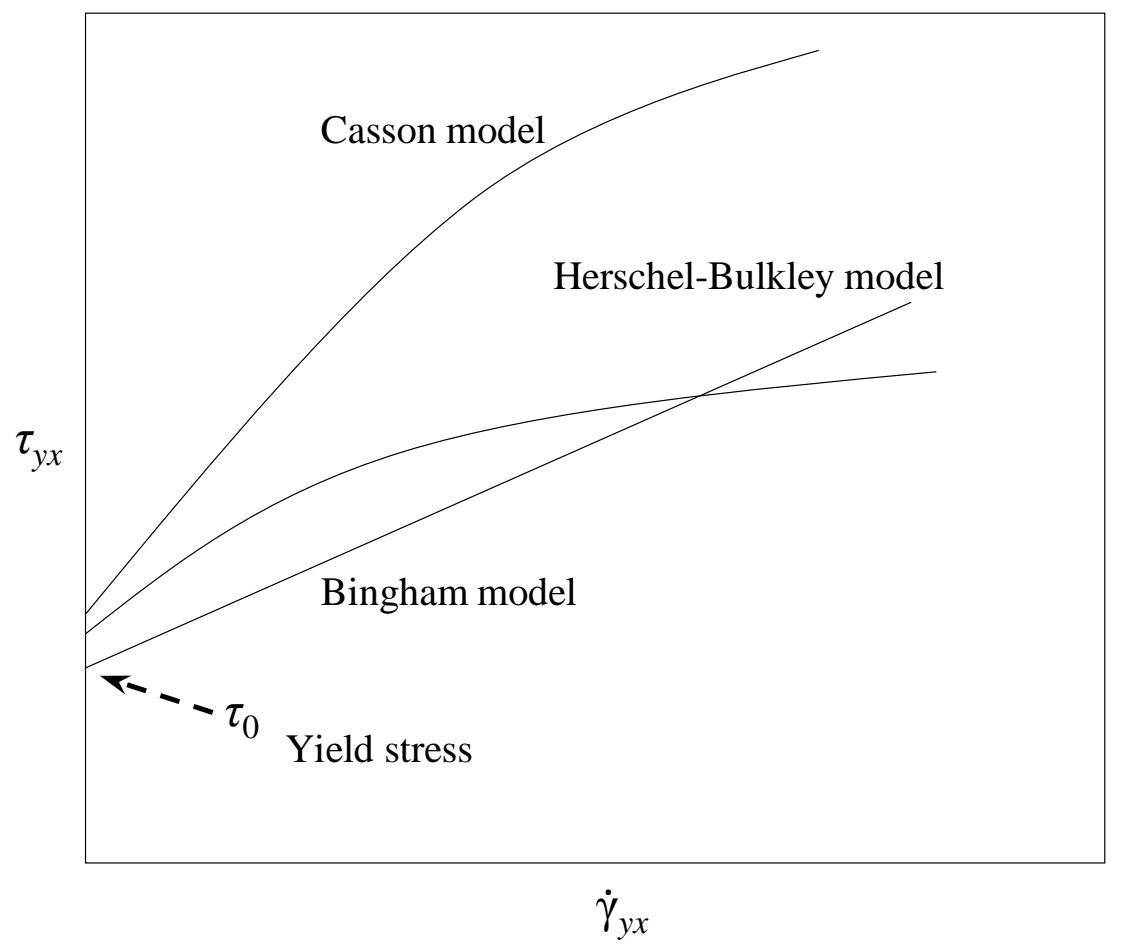

Source: Adapted from Chhabra and Richardson (2011).

\subsubsection{Laminar Flow in Circular Pipes}

Consider a steady, incompressible and fully developed flow of a viscoplastic fluid in a circular pipe of radius, $R$. Because, there is no angular velocity, the force balance in the z-direction in an infinitesimal fluid element situated at distance, $r$, as it is presented in figure 2.4, can be expressed as the balance between friction and pressure forces, as follows (CHHABRA; RICHARDSON, 2011):

$$
P\left(\pi r^{2}\right)-(P+\Delta P) \pi r^{2}=\tau_{r z} 2 \pi r L
$$

solving for $\tau_{r z}$,

$$
\tau_{r z}=\left(\frac{-\Delta P}{L}\right) \frac{r}{2}
$$

where the subscript $r z$ means the direction normal and parallel to the forces and shear stress.

These equations show the linear stress distribution across the pipe cross-section, the shear stress being zero at the central axis of the tube, figure 2.5. The equation (2.8) is applicable to laminar and turbulent flow conditions, since it is based on a force balance and no assumption different has been made so far concerning the type of flow or fluid behavior.

As it was mentioned, an yield stress fluid will flow only if the applied stress exceeds the yield stress. There is a solid plug-like core that flows in the middle of the pipe, where $\left|\tau_{r z}\right|$ is smaller than the yield stress. The radius of the plug $R_{p}$ depends o the yield stress and the wall shear stress. That means that depends on the expression $-\Delta P / L$. From the equation (2.8), a relation between the yield and wall shear 
Figure 2.4 - Force balance in a flow through a horizontal pipe.

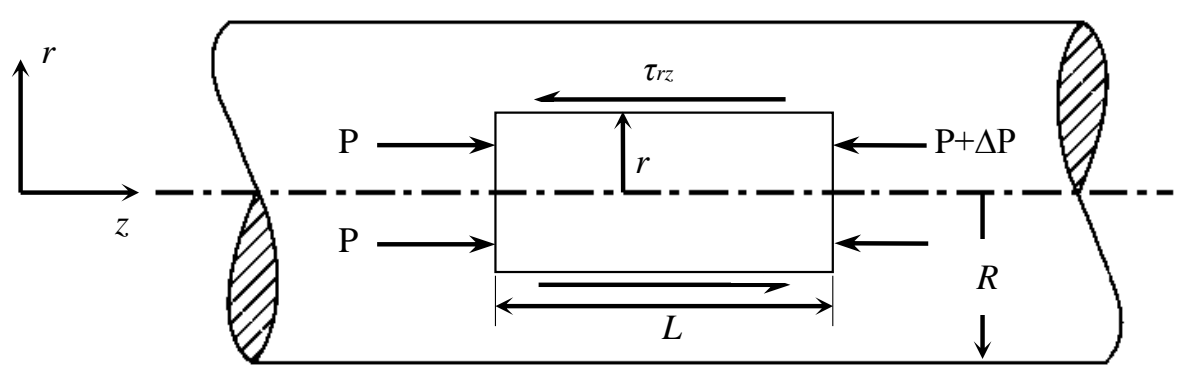

Source: Adapted from Chhabra and Richardson (2011).

stress can be expressed as:

$$
\frac{\tau_{0}}{\tau_{w}}=\frac{R_{p}}{R}
$$

where $\tau_{w}$ is the shear stress at the pipe wall.

Inside the annular area between $R_{p}$ and $R$ in figure 2.5 , the velocity gradually will decrease from the plug at constant velocity to zero at the pipe wall. Additionally, in this region the value of the shear stress will be greater than the yield stress, and the Bingham model given by the equation (2.3) can be written as:

$$
\tau_{r z}=\tau_{0}^{B}+\eta_{B}\left(-\frac{d u_{z}}{d r}\right)
$$

Combining, equations (2.8) and (2.10) and integrating with respect to $r$, it results in the following expression for the velocity distribution:

$$
u_{z}=-\frac{1}{\eta_{B}}\left\{\left(-\frac{\Delta P}{L}\right) \frac{r^{2}}{2}-r \tau_{0}^{B}\right\}+C
$$

where $C$ is a constant of integration.

At the walls of the circular pipe, when $r=R$, the velocity $u_{z}$ must be zero in order to satisfy the no-slip condition. Substituting this value by $u_{z}$ at $r=R$ in the equation (2.11), the value obtained for the constant is:

$$
C=\frac{1}{\eta_{B}}\left\{\left(-\frac{\Delta P}{L}\right) \frac{R^{2}}{2}-R \tau_{0}^{B}\right\}
$$

Finally the velocity distribution in the region between $R_{p}<r<R$ is described by:

$$
u_{z}=\left(-\frac{\Delta P}{L}\right) \frac{R^{2}}{4 \eta_{B}}\left(1-\frac{r^{2}}{R^{2}}\right)-\frac{\tau_{0}^{B}}{\eta_{B}} R\left(1-\frac{r}{R}\right)
$$

The equation 2.13 is applicable when $\left|\tau_{r z}\right|>\left|\tau_{0}^{B}\right|$ and $r \geq R_{p}$. The velocity in the plug region, $0 \leq r \leq R_{p}$ is obtained by substituting $r=R_{p}$, in the equation (2.13):

$$
u_{z p}=\left(-\frac{\Delta P}{L}\right) \frac{R^{2}}{4 \eta_{B}}\left(1-\frac{R_{p}}{R}\right)^{2}
$$

For the volumetric flow rate, $Q$, is obtained by evaluating the integral

$$
Q=\int_{0}^{R} 2 \pi r u_{z} d r=\int_{0}^{R_{p}} 2 \pi r u_{z} d r+\int_{R_{p}}^{R} 2 \pi r u_{z} d r
$$


Figure 2.5 - Velocity distribution for laminar flow of a viscoplastic fluid in a pipe.

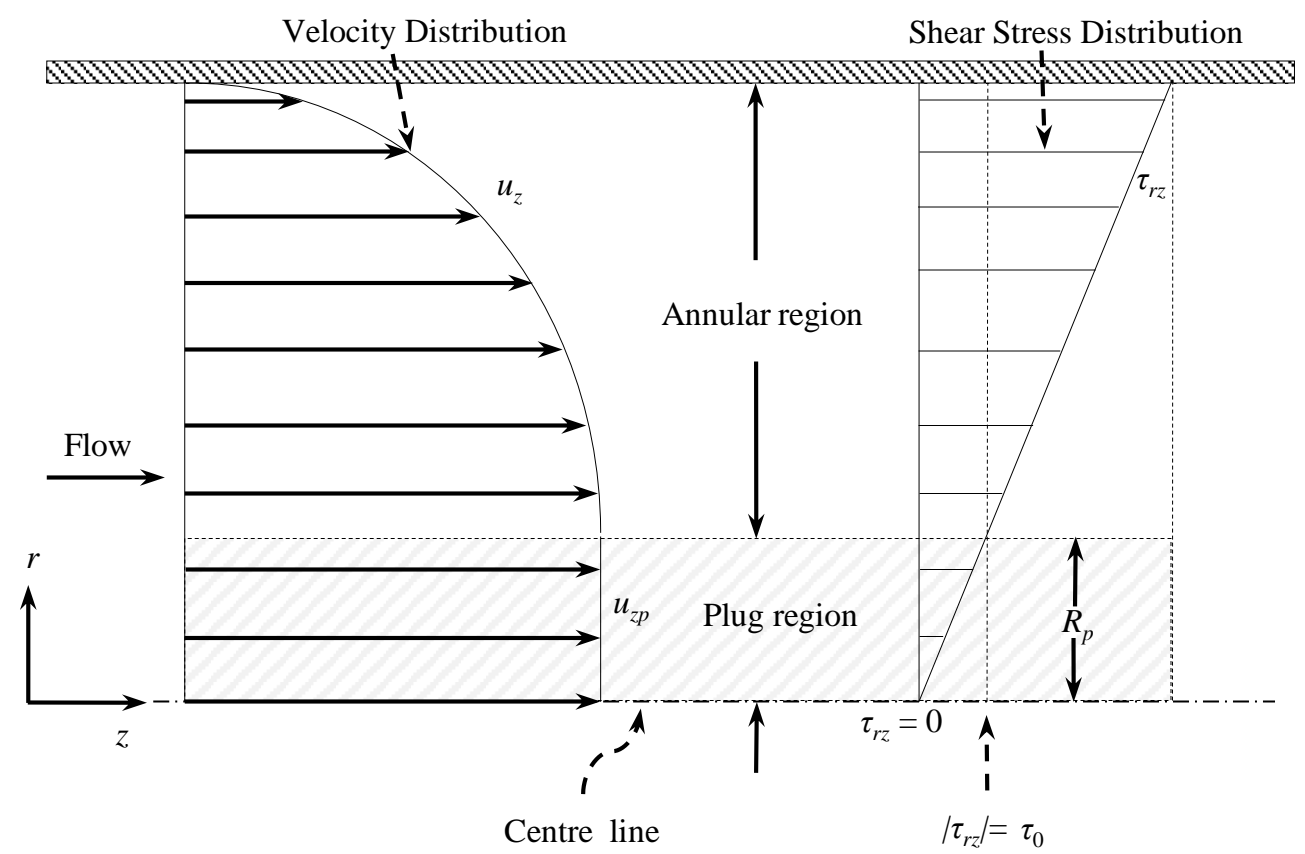

Source: Adapted from Chhabra and Richardson (2011).

Substituting from equations (2.14) and (2.13):

$$
\begin{aligned}
Q= & \int_{0}^{R_{p}}\left(-\frac{\Delta P}{L}\right)\left(-\frac{R^{2}}{4 \eta_{B}}\right)\left(1-\frac{R_{p}}{R}\right)^{2} 2 \pi r d r \\
& +\int_{R_{p}}^{R}\left[\left(-\frac{\Delta P}{L}\right)\left(-\frac{R^{2}}{4 \eta_{B}}\right)\left(1-\frac{r^{2}}{R^{2}}\right)-\frac{\tau_{0}^{B} R}{\eta_{B}}\left(1-\frac{r^{2}}{R^{2}}\right)\right] 2 \pi r d r
\end{aligned}
$$

Evaluating the integrals and denoting the ratios $\tau_{0}^{B} / \tau_{w}=R_{p} / R=\phi$, the equation (2.16) is reduced to:

$$
Q=\frac{\pi R^{4}}{8 \eta_{B}}\left(-\frac{\Delta P}{L}\right)\left[2 \phi^{2}(1-\phi)^{2}+2\left(1-\phi^{2}\right)-\left(1-\phi^{4}\right)-8 \phi\left(\frac{1-\phi^{2}}{2}-\frac{1-\phi^{3}}{3}\right)\right]
$$

with further simplifications it leads to:

$$
Q=\frac{\pi R^{4}}{8 \eta_{B}}\left(-\frac{\Delta P}{L}\right)\left(1-\frac{4}{3} \phi+\frac{1}{3} \phi^{4}\right)
$$

This equation can be rewritten in a dimensionless expression introducing dimensionless numbers for Bingham fluids, defined as:

Reynolds number based on the apparent viscosity found by Bingham model,

$$
\operatorname{Re}_{B}=\frac{\rho \bar{U} D}{\eta_{B}}
$$

Friction factor,

$$
f=\frac{\tau_{w}}{\left(\frac{1}{2}\right) \rho \bar{U}^{2}}
$$


Hedström number,

$$
H e_{B}=\frac{\rho D^{2} \tau_{0}^{B}}{\eta_{B}^{2}}
$$

where $D$ is the pipe diameter. $H e_{B}$ number is a dimensionless define by Hedström (1952) to obtain the turbulent region for the plastic fluids. This number is define by correlations obtained by the friction factor and the Reynolds Number, and as its value increase means that the critical region to reach a turbulent state moves away from the critical Newtonian Reynolds number. Originally this number was defined in function of the plastic characteristics given by the rheological properties of Bingham fluids. Then, its definition was extended in a general function for viscoplastic fluids, as will be shown below.

From the Eqs. (2.19), (2.20), (2.21), and the ratio $\phi=\left(\frac{\tau_{0}^{B}}{\tau_{w}}\right)=\left(\frac{\tau_{0}^{B}}{(1 / 2) f \rho \bar{U}^{2}}\right)$, the equation (2.18) can be rewritten in terms of the mean velocity, $\bar{U}=Q / \pi R^{2}$, as follows:

$$
\bar{U}=\frac{(D / 2)^{2}}{8 \eta_{B}}\left(\frac{-\Delta P}{L}\right)\left\{1-\frac{8}{3} \frac{\tau_{0}^{B}}{f \rho \bar{U}^{2}}+\frac{1}{3}\left(\frac{2 \tau_{0}^{B}}{f \rho \bar{U}^{2}}\right)^{4}\right\}
$$

Rearranging leads to:

$$
\frac{\eta_{B}}{\rho \bar{U} D}=\frac{(D / 2)^{2}}{32 \rho \bar{U}^{2}}\left(\frac{-\Delta P}{L}\right)\left\{1-\frac{8}{3} \frac{\tau_{0}^{B}}{f \rho \bar{U}^{2}}+\frac{16}{3}\left(\frac{\tau_{0}^{B}}{f \rho \bar{U}^{2}}\right)^{4}\right\}
$$

i.e.,

$$
\frac{1}{R e_{B}}=\frac{f}{16}\left\{1-\frac{8}{3} \frac{\tau_{0}^{B}}{f \rho \bar{U}^{2}}+\frac{16}{3}\left(\frac{\tau_{0}^{B}}{f \rho \bar{U}^{2}}\right)^{4}\right\}
$$

A final form for $f$ can be obtained:

$$
f=\frac{16}{R e_{B}}\left(1+\frac{1}{6} \frac{H e}{R e_{B}}-\frac{1}{3} \frac{H e^{4}}{f^{3} R e_{B}^{7}}\right)
$$

The equation (2.25) is implicit in friction factor and therefore, for a specified flow rate is needed an interactive method in order to evaluate the pressure drop. Some predictions can be found at Chhabra and Richardson (2011), where it is showed that for low Hedström numbers the fluids reach a Newtonian behavior, i.e., $f=16 / R e_{B}$. Also, it is appropriated to add another dimensionless parameter, Bingham number, $B i$, defined as:

$$
B i=\frac{\tau_{0}^{B} D}{\eta_{B} \bar{U}}
$$

and a relation between the Hedström and Bingham number can be expressed by $H e=R_{B} B i$ (MALIN, 1998; CHHABRA; RICHARDSON, 2011). Therefore, the equation (2.25) can be cast in terms of the Bingham number:

$$
f=\frac{16}{R e_{B}}\left[1+\frac{B i}{6}-\frac{1}{3} \frac{B i^{4}}{\left(f R e_{B}\right)^{3}}\right]
$$

The value of $f R e_{B}$ deviates a little from its Newtonian value of 16 up to $B i \sim 0.1$, so a little effect of the yield stress of the fluid on the pressure gradient can be suggested. It is convenient to calculate the pressure gradient for a given flow rate, pipe diameter and rheological characteristics $\left(\eta_{B}, \tau_{0}^{B}\right)$; the reverse calculation of a unknown flow rate for a given pressure gradient requires an interactive solution (CHHABRA; RICHARDSON, 2011; MALKIN, 1994). 
This analytical framework can be extended for laminar flow of Herschel-Bulkley model fluids, and final expression for the mean velocity, the plug velocity and the volumetric flow rate are given as (SKELLAND, 1967; BIRD et al., 1983; BIRD et al., 1987):

$$
u_{z}=\frac{n R}{(n+1)}\left(\frac{\tau_{w}}{m}\right)^{1 / n}\left\{(1-\phi)^{(n+1) / n}-\left(\frac{r}{R}-\phi\right)^{(n+1) / n}\right\}
$$

The plug velocity, $u_{z p}$ is given as:

$$
u_{z p}=\frac{n R}{(n+1)}\left(\frac{\tau_{w}}{m}\right)^{1 / n}(1-\phi)^{(n+1) / n}
$$

and the volumetric flow rate $Q$, as:

$$
Q=\pi R^{3} n\left(\frac{\tau_{w}}{m}\right)^{1 / n}(1-\phi)^{(n+1) / n}\left\{\frac{(1-\phi)^{2}}{3 n+1}+\frac{2 \phi(1-\phi)}{2 n+1}+\frac{\phi^{2}}{n+1}\right\}
$$

where $\phi$ for Herschel-Bulkley case is the ratio $\tau_{0}^{H} / \tau_{w}$. As the Bingham case, these expressions are implicit in pressure gradient.

Also, some dimensionless relations can be described for fluids through the Herschel-Bulkley model. Malin (1998) expressed that the Hedström number for Herschel-Bulkley fluids can be given by the general equation:

$$
H e_{H B}=\frac{\rho D^{2} \tau_{0}^{H}{ }^{(2 / n-1)}}{m^{2 / n}}
$$

$H e_{H B}$ is a relation between the yield stress and the viscous stress for a given geometry. Also, a generalized Bingham number can be expressed to described viscoplastic fluids. This expression is knows as Herschel-Bulkley number (HB). It allows to relate the yield and the viscous stress by the expression (ALEXANDROU et al., 2003):

$$
H B=\frac{\tau_{0}^{H} D^{n}}{m \bar{U}^{n}}
$$

When the viscosity varies as the fluid is deformed, an appropriate viscosity is necessary to define a generalized Reynold number for the different viscoplastic fluid models. For Herschel-Bulkley fluids it is easier to calculate some parameters when the mean wall viscosity is chosen $\left(\eta_{w}\right)$. The mean wall viscosity is calculated from $\tau_{w}$, and using the Eq. (2.8), $\tau_{w}$ can be expressed as:

$$
\tau_{w}=\left(\frac{-\Delta P}{L}\right) \frac{D}{4}
$$

so, for a Herschel-Bulkley rheology (RUDMAN et al., 2004), $\eta_{w}$ can be expressed as:

$$
\eta_{w}=m^{1 / n} \frac{\tau_{w}}{\left(\tau_{w}-\tau_{0}^{H}\right)^{1 / n}}
$$

then, the expression of $R e$ can be generalized for non-Newtonian fluids as:

$$
R e_{g}=\frac{\rho \bar{U} D}{\eta_{w}}
$$


The equations (2.33), (2.34) can be replaced at Eq. (2.35), and its obtained a new general expression for $R e$, given by:

$$
R e_{g}=\frac{\rho \bar{U}^{2-n} D^{n}}{m}
$$

This expression is the most suitable to calculate the $R e$ for Herschel-Bulkley fluids at laminar regime. According to Durst et al. (1995) this expression allows to calculate the entrance length $\left(L_{e}\right)$, after which the laminar flow of a Herschel-Bulkley fluid is considered fully developed. This relation was expressed as:

$$
\frac{L_{e}}{R} \frac{1}{R e_{g}}=\frac{0.23}{n^{0.31}}-0.4 \phi
$$

where from the equation (2.17) $\phi$ is defined by $R_{p} / R$ or $\tau_{0}^{H} / \tau_{w}$.

\subsubsection{Friction Factors in Transitional and Turbulent Flow Conditions}

As the same way that there are equations for predicting friction factor for turbulent Newtonian flow, there are equations for non-Newtonian time-independent fluids. Mostly are based on experimental observations (GOVIER; AZIZ, 1972; HEYWOOD; CHENG, 1984).

Dodge and Metzner (1959) carried out a semi-empirical analysis of the fully developed turbulent flow of power-law fluids in smooth pipes, and obtained an expression which can be rearranged in terms of the flow behavior index $n$ (also knows as power law index), as follows:

$$
\frac{1}{\sqrt{f}}=A(n) \log \left(R e_{M R} f^{(2-n) / 2}\right)+C(n)
$$

where $A(n)$ and $C(n)$ are two unknown functions of $n$. Based on extensive experimental results in the range $2900 \leq R e_{M R} \leq 36000 ; 0.36 \leq n \leq 1$ for polymer solutions and particulate suspensions, these function are expressed by:

$$
\begin{gathered}
A(n)=4(n)^{-0.75} \\
C(n)=-0.4(n)^{-1.2}
\end{gathered}
$$

and the $R e_{M R}$ is the Reynold number stablished by Metzner and Reed (1955) correlations, which is defined as:

$$
\operatorname{Re}_{M R}=\frac{\rho \bar{U}^{2-n^{\prime}} D^{n^{\prime}}}{m^{\prime} 8^{n^{\prime}-1}}
$$

where

$$
\begin{gathered}
n^{\prime}=\frac{(1-\phi)+2 \phi(1-\phi)(1+a) /(2+a)+(1-\phi)^{2}(1+a) /(3+a)}{a+1-3(1-\phi)\left[\phi^{2}+2 \phi(1-\phi)(1+a) /(2+a)+(1-\phi)^{2}(1+a) /(3+a)\right]} \\
m^{\prime}=\left(\frac{m^{a}}{4}\right)^{n^{\prime}}\left(\frac{\tau_{0}}{\phi}\right)^{1-n^{\prime} a}\left\{(1-\phi)^{1+a}\left[1+\frac{2(1-\phi)(1+a)}{\phi(2+a)}+\frac{(1-\phi)^{2}(1+a)}{\phi^{2}(3+a)}\right]\right\}^{-n^{\prime}}
\end{gathered}
$$


and $a$ is defined as $1 / n$. The expressions for $n^{\prime}$ and $m^{\prime}$ indicate the dependence of these values on the rheological parameters and the plug ratio $\phi$.

Now, substituting the equations (2.39) and (2.40) for the constants in the equation (2.38),

$$
\frac{1}{\sqrt{f}}=\frac{4}{n^{0.75}} \log \left(R e_{M R} f^{(2-n) / 2}\right)-\frac{0.4}{n^{1.2}}
$$

The equation (2.44) is applied to all kinds of time-independent fluids. Despite this, numerous authors have presented expressions for turbulent flow friction factors for specific models. Tomita (1959) applied the concept of the Prandtl mixing length and modified definitions on the friction factor and Reynolds number for turbulent flow of Bingham plastic fluids in smooth pipes. The applicability of this method was tested using data in the range $2000 \leq \operatorname{Re}_{B}(1-\phi)^{2}(3-\phi) \leq 10^{5}$.

Nevertheless, there are experimental correlations that allow obtaining the most suitable Reynolds number expressions for Herschel-Bulkley fluids based on the limitations of the $R e_{M R}$ for a wide range of $n$ and Reynolds numbers. Such as Malin (1998) and Madlener et al. (2009), they established experimental correlations focused on the laminar and turbulent flow conditions.

Malin (1998) stablished correlations from experimental and numerical simulations, and determined the friction factor, obtaining a relationship between $n, R e_{g}$ and $H e$. The obtained expression for a general Reynolds number is given by:

$$
R e^{\prime}=\frac{\rho \bar{U}^{2-n} D^{n}}{m(0.75+0.25 / n)^{n} 8^{n-1}}
$$

The experimental friction factors obtained by Malin (1998) for the laminar regime were represented by the analytical data expressed by the following equation:

$$
f=\frac{16}{\Psi R e^{\prime}}
$$

where $\Psi$ is defined by:

$$
\Psi=(1+3 n)^{n}(1+\phi)^{(1+n)}\left[\frac{(1-\phi)^{2}}{(1+3 n)}+\frac{2 \phi(1-\phi)}{(1-2 n)}+\frac{\phi^{2}}{(1+n)}\right]
$$

On the other hand, for the turbulent regime, $f$ is based on the correlations described by Metzner and Reed (1955) and Dodge and Metzner (1959), given by:

$$
\frac{1}{\sqrt{f}}=\frac{4}{n^{0.75}} \log \left[R e^{\prime} f^{(2-n) / 2}\right]-\frac{0.4}{n^{1.2}}
$$

which is much simpler to use than the complex correlations expressed by Hanks et al. (1967).

\subsubsection{Flow Structure of Viscoplastic Flow in Contractions}

The flow of a viscoplastic fluid through contractions is distinguished from other non-Newtonian fluids by the existence of a plug region due to shear stress values smaller than the yield stress, as was expressed in section 2.1. Also, it is marked by the appearance of vortex structures and unyielded regions at the corners of the contraction. Moreover, in some cases, both conditions coexist as represented in Fig. 2.6. 
Figure 2.6 - Flow of viscoplastic fluid through a contraction. Points 1, 2, 3, and 4 presents the velocity profile changes along the contraction.

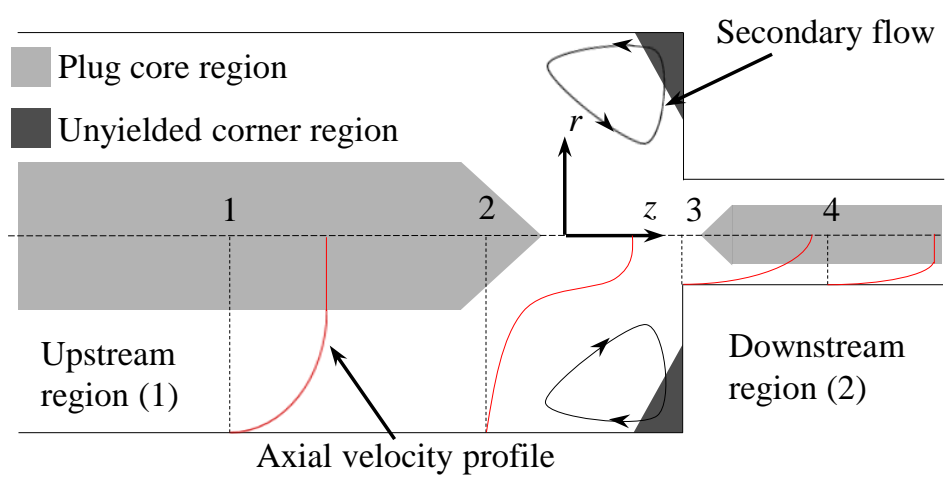

Source: Author (2019).

The behavior of the viscoplastic fluid flows in contractions can be explained by the Cauchy stress tensor $(\sigma)$ definition. This is composed of the hydrostatic stress tensor $(\mathrm{p} \delta)$ and the shear stress $(\tau)$, known as the deviatoric part, which is responsible for the deformation of the material (PRAGER, 1952). As the model for viscoplastic fluid behavior is based on the Von Mises yield criterion, it is argued that the shear stress is zero on the axis of symmetry, and the main stresses for the material are defined as $\sigma_{11}=\sigma_{22}$ and $\sigma_{22}-\sigma_{33}= \pm \sqrt{\tau_{0}}$ (HILL, 1950). Near to the solid boundary, there is a stagnation point, because it is considered the no-slip condition at the wall, and it is assumed incompressibility. Hence the stagnation point is a point of zero deviatoric stress; it means that the hydrostatic stress of the fluid counteracts the main stresses, and the material is clearly unyielded due to is no deformation or distortion at this point. Away from this point, along the axis of symmetry, the stress changes in a continuous manner, governed in any unyielded regions by the "elastic" response, until the yield criterion is exceeded (TANNER, 2000).

\subsubsection{Axial velocity profile}

The structure of the flow presented in Fig. 2.6 shows that as the plane of the contraction is approached, the flow accelerates rapidly, and the centerline velocity increases. When the flow enters the smallest diameter, the centerline velocity increases and the flow can reach fully developed conditions (VRADIS; HAMMAD, 1996), or in some cases changes its flow regime. The reason for this behavior is the fact that the region near the contraction is characterized by high shear rates and the shape of profile changes along the upstream region from a flat profile to a parabolic one (HAMMAD; VRADIS, 1996).

\subsubsection{Plug core region}

As described in the last subsections and showed in Fig. 2.6, the structure of the flow is divided mainly by the central plug region at the upstream and downstream of the flow-loop. For low $H B$, the size of the downstream plug core is small, and at the upstream region, the plug core takes roughly $60-70 \%$ of the circular area (JAY et al., 2002). The $H B$ is increased, large plug core diameters occur at the downstream and upstream regions. Hence, the $H B$ is a dimensionless number proportional to $\tau_{0}$ and inversely proportional to the mean velocity $(\bar{U})$ according to Eq. (2.32); high mean velocities means low $H B$ numbers, and high shear rates with high $\tau_{0}$ means larger $H B$ numbers (JAY et al., 2002). 


\subsubsection{Unyielded corner regions and vortex structures}

Unyielded regions at the corners of the contraction can appear in a viscoplastic fluid flow structure. Different studies have shown that as the $H B$ is increased, the unyielded regions are gradually replaced by vortex structures (NGUYEN; BOGER, 1979). For example, a $H B=0$ (Newtonian fluid) does not present unyielded regions, and on the contrary, the corner is entirely occupied by a vortex structure. For some cases with low $H B$ and transitional $R e_{D}^{\prime}$, it is possible that two unyielded regions appear, and coexist with a vortex structure according to Jay et al. (2002). Also, in this numerical study was shown that as the flow velocity decreases, a single larger rigid static zone appears at the corner regions, until the vortex structure disappears.

\subsubsection{Aspect ratio}

Numerical studies have shown that changes in unyielded regions occur, when $\beta$ increases the size of the unyielded regions at the corners also increases (JAY et al., 2002). The extremity of the upstream core plug zone is pushed back, but for the downstream region, there is no significantly changes.

Another important parameter in the geometry aspect is the contraction angle. The numerical study of Jay et al. (2002) shows the contraction angle affects the unyielded size regions at the corner of the viscoplastic fluid flow. Thus, these regions decrease drastically as the angle decreases, and the upstream plug region is pushed backward.

\subsubsection{Pressure loss coefficient}

Another relevant characteristic of the fluid flow through contractions is the pressure drop when the mean velocity changes due to the passage to a small section area (FESTER et al., 2008). As expressed before, the sudden change of velocity produces energy losses resulting in pressure losses. For this case of flow through a contraction, the pressure loss depends on the geometry and the Reynolds number. This localized pressure loss can be expressed by (FESTER et al., 2008):

$$
h_{l}=k \frac{\bar{U}^{2}}{2 g}
$$

where $h_{l}$ is the localized pressure loss. It is possible expresses this equation for $k$ in terms of pressure drop $(\Delta P)$ between two points along the contraction:

$$
k=\frac{2 \Delta p}{\rho \bar{U}^{2}}
$$

The equation (2.50) is based on the static pressure only. In the case of contractions where the upstream and downstream velocities are different, the total pressure should be used to account for changes in kinetic energy (FESTER et al., 2008):

$$
k_{\text {con }}=\frac{\left(\Delta P_{\text {con }} / \rho g\right)+\left(\alpha_{1} \bar{U}_{1}^{2}-\alpha_{2} \bar{U}_{2}^{2}\right) / 2 g}{\bar{U}_{2}^{2} / 2 g}
$$

the subscripts 1 and 2 mean the upstream and downstream region of the contraction, respectively, represented at figure 2.6. The Greek letter $\alpha$ is the kinetic energy correction factor for fully developed profiles. 
For laminar flow condition, this factor is defined using the correlation calculated by Mika (2011), which is based on the shear stress ratio $\phi$ :

$$
\alpha=\frac{54\left(47 \phi^{2}+58 \phi+35\right)}{35\left(\phi^{2}+2 \phi+3\right)^{3}}
$$

when $\phi=0\left(R_{p}=0\right)$, the Eq. (2.52) reaches the value $\alpha=2$, which is the value for laminar Newtonian flow. And for $\phi=1\left(R_{p}=R\right)$ its value is $\alpha=2$, that means the entire velocity profile reaches the maximal velocity or the core plug velocity, and it is the value of $\alpha$ for turbulent Newtonian flow.

For turbulent coditions Strzelecka and Jezowiecka-Kabsch (2008) presented some corrections to determine these factor based on the method of Szewczyk (2008), given by:

$$
\alpha=1+101\left(\frac{10}{\ln ^{2} R e}\right)^{3}-10.7\left(\frac{10}{\ln ^{2} R e}\right)^{2}+\frac{11.3}{\ln ^{2} R e}
$$

The equation (2.53) is valid for $4900 \leq R e$. For $2800 \leq R e \leq 1000000$ range, the Eq. (2.53) was modified by Strzelecka and Jezowiecka-Kabsch (2008), which is used to calculate kinetic energy correction factors in turbulent flow range:

$$
\alpha=1+105\left(\frac{10}{\ln ^{2} R e}\right)^{3}-11.88\left(\frac{10}{\ln ^{2} R e}\right)^{2}+1.208\left(\frac{11.3}{\ln ^{2} R e}\right)
$$

For the turbulent regime, $\alpha$ reaches values close to 1 for viscoplastic fluid flows. Similar to the case of turbulent Newtonian flows, $\alpha$ is approximately 1 due to a small variation of the kinetic energy (PRITCHARD; MITCHELL, 2016). Then, it is possible to state that for turbulent conditions, $\alpha$ presents similar values for both types of fluids, Newtonian and non-Newtonian ones.

\subsection{FUNDAMENTAL PRINCIPLES OF PARTICLE IMAGE VELOCIMETRY (PIV)}

Experimental studies focused on flow visualization require some special conditions to be performed as transparent experimental setup. Quantitative information of the flow can be obtained using some equipment as rotameter and Venturi-meter. However, only some average properties can be obtained in these devices, and also these techniques are intrusive, modifying the measured variable and sometimes altering the flow conditions.

Spatial correlations in the velocity can be obtained through the PIV technique, when it is not possible to use point measurement techniques, such as LDV (Laser Doppler Velocimetry). The PIV system consists of setting up several subsystems, in which the principal is a pulsed laser source and optics devices to produce a laser sheet. The laser generates a high power illumination to obtain good images resolution. The images of the particles are determined at two instants of time using a CCD (Charged Couple Device) or a CMOS (Complementary Metal-Oxide- Semiconductor) camera. Then, the images are transferred to software for processing and obtaining velocity fields (DESHPANDE, 2010b; RAFFEL et al., 2018).

PIV is a non-intrusive technique that allows measuring local quantitative information. Using local mean values at a point or region the spatial velocity variation can be determined. Thus with this technique, 
it is possible to evaluate the coupling between velocities at different points of the flow field, and obtain the spatial distribution of the velocity, for example, the parabolic profile of laminar flow under steady fully developed conditions (DESHPANDE, 2010b).

The instantaneous velocity of a group of seeded particles can be provided in a cross-section of flow using the PIV technique. Two consecutive light pulses illuminate the test section area with a short duration produced by a laser. Usually, the type of laser used for these applications is a double-pulsed Nd: Yag (Neodymium-doped Yttrium aluminum garnet). The duration of the light pulses and the time between pulses $(\Delta t)$ is typical of the order of microseconds $(\mu s)$. The time depends on velocities display in the test section, also on the acquisition parameters and the type of laser used. It is important to know the velocity of the particles, and it can be estimated by finding the displacement $(\Delta x, \Delta y)$ of the particles between the laser pulses in $x$ and $y$ direction. Since the time interval between the laser pulses is known, and the camera is calibrated to get the displacement of the object, the flow velocity is determined as follows:

$$
\begin{aligned}
& u_{x}=\lim _{\Delta t \rightarrow 0} \frac{\Delta x}{\Theta \Delta t} \\
& u_{y}=\lim _{\Delta t \rightarrow 0} \frac{\Delta y}{\Theta \Delta t}
\end{aligned}
$$

where $\Theta$ is the measure scale factor of the camera, that depends on the calibration of the target area dimensions.

Figure 2.7 - Experimental arrangement for particle image velocimetry and correlation process.

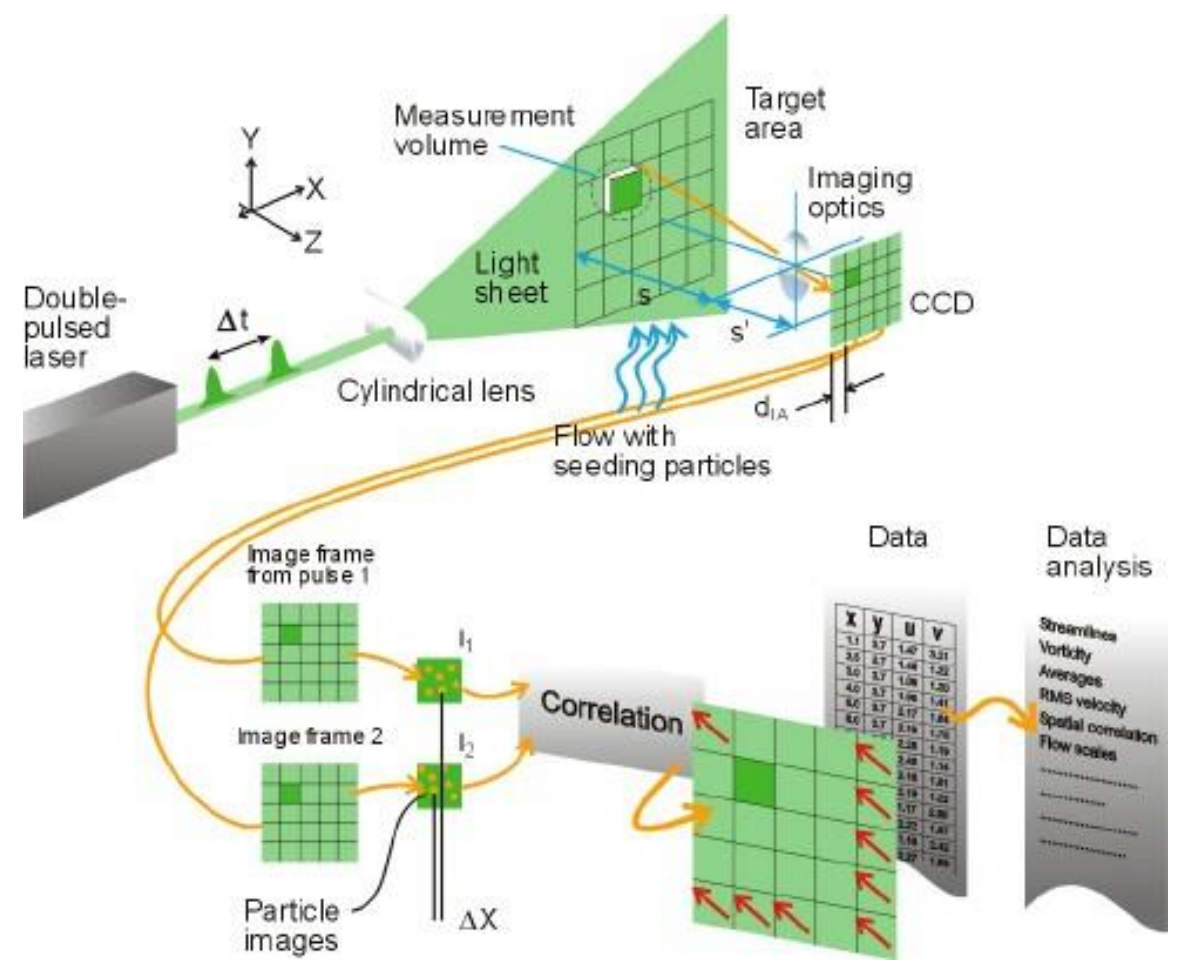

Source: Adapted from Dynamics (2015).

The entire image from the camera is divided into small sub-regions named interrogation areas (IA) or "target areas", and the local displacement of the particles is determined within each interrogation area, as 
it is shown in Fig. 2.7. In each interrogation area, all the particles are assumed to have the same velocity, and with a correlation technique is determined the displacement using the information from a couple of frames taken by the camera.

An important fact is that increasing the number of interrogation windows results in a higher resolution, as long as the correlation is not be affected by signal noise. A properly distribution and density of tracer particles in the flow are essential to allow a higher resolution and to decrease the noise in the images. Regarding the density of tracer particles, it is necessary to know that three different types of image density can be distinguished, as illustrated in figure 2.8. For low densities of particles, it is necessary to use tracking methods for evaluation, this is referred to the Particle Tracking Velocimetry (PTV), here the images of individual particles can be detected and images that correspond to the same particle and originate from different illuminations can be identified. For medium image density, the images of individual particles can be detected as well. However, it is not possible to identify a pair of images by visual inspection of the recording. For medium particle density is possible to apply the standard PIV evaluation techniques. Finally, for high particle density is not possible to detect individual images as they overlap in most cases and form speckles (RAFFEL et al., 2018). So, for high densities are required other techniques as Laser Speckle Velocimetry (LSV), which is more suitable for high tracer particle density.

Figure 2.8 - Particle density in laser applications techniques: (a) low (PTV), (b) medium (PIV), (c) high particle density (LSV).

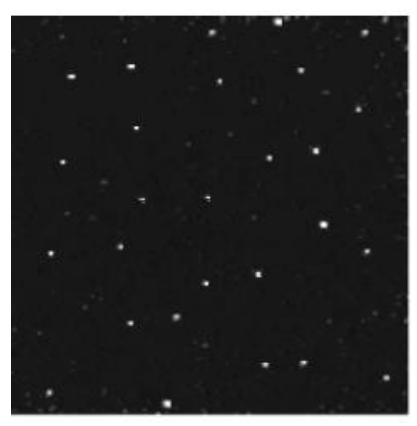

(a)

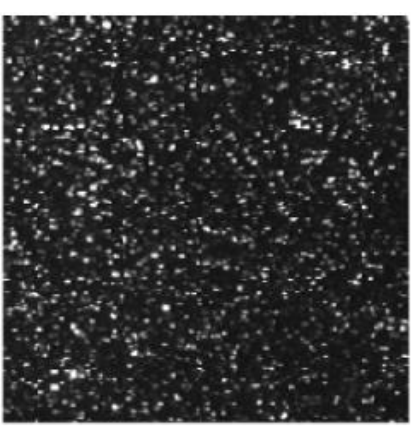

(b)

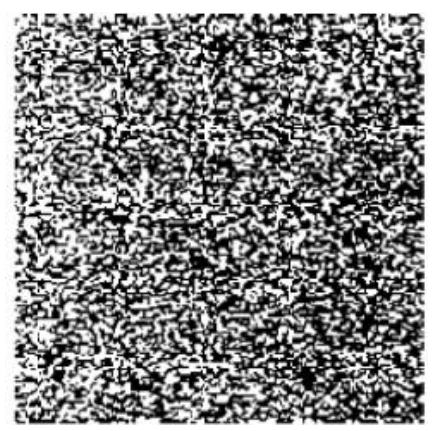

(c)

Source: Taken from Raffel et al. (2018).

\subsection{CHAPTER SUMMARY}

From this chapter basic theory about non-Newtonian fluids behavior, specially the behavior of viscoplastic fluids and the different models which describe and characterize the rheological properties of these fluids: Bingham and Herschel-Bulkley models as the most important. Also, the flow of these type of fluids was presented at different regimes and the parameters for each flow condition: laminar, transitional and turbulent regime, and they were correlated with dimensionless parameters as Hedstrom, Bingham and Herschel-Bulkley number at the means equations for flow into circular pipes.

Finally a brief explanation of PIV technique was made, in order to highlight the importance of using this technique for the visualization of fluid flows in experimental studies, instead of others techniques as LDV, LSV and PTV. 


\section{LITERATURE REVIEW}

In this chapter, a review of viscoplastic fluids flow in straight tubes, and abrupt contractions is presented. Subsequently, relevant studies involving turbulent flows in these conditions are discussed. Lastly, this chapter explains the use of the PIV technique for visualizing the dynamics of viscoplastic and Newtonian fluids in different pipe configurations.

\subsection{FLOW OF VISCOPLASTIC FLUIDS IN STRAIGHT TUBES}

Many numerical studies of non-Newtonian fluid flow in turbulent regime were performed to understand its behavior. Rudman et al. (2004) performed a relevant study for non-Newtonian fluids: Herschel-Bulkley and power law fluids. From this study the mean flow quantities and the first order turbulent quantities for each non-Newtonian fluid were obtained. For Herschel-Bulkley fluids data were compared with experimental results obtained with $0.05 \%$ wt Carbopol ${ }^{\circledR}$ Ultrez 10 solution with rheological properties: $\tau_{0}^{H}=1.35 \mathrm{~Pa}, m=1.203 \mathrm{~Pa} \cdot \mathrm{s}^{n}$ and $n=0.52$. The mean flow quantities were normalized using the centerline velocity $\left(u_{C L}\right)$ and for turbulent quantities, the mean wall velocity $\left(u_{w}\right)$, and mean wall viscosity $\left(\eta_{w}\right)$. Rudman et al. (2004) expressed that even though the DNS is the most suitable numerical simulation to obtain reliable results, there is a percent of error when they are compared to the experimental data due to the difficulties when it is necessary to control the rheological properties of the fluid. There was a difference of 10\%-15\% for the friction factor obtained by experimental correlation for $R e=3025$ and 3840. Velocity fluctuations were showed for the experimental tests, due to a transitional character of the flow and the low yield stress for the Herschel-Bulkley fluid solution. Nevertheless, it was emphasized that non-Newtonian fluids are difficult to approximate measured rheology and affect the accuracy of the results compared with DNS data.

Peixinho et al. (2005) performed an experimental study to analyze the different flow regimes, laminar, transitional, and turbulent. Using a Laser Doppler Anemometer technique (LDA), the experimental study was performed for three different fluids: a yield stress aqueous solution of $0.2 \%$ wt Carbopol as viscoplastic fluid, an aqueous solution of $2 \% w t$ CMC as pseudoplastic and glucose syrup as a Newtonian fluid. The viscoplastic fluid was modeled by Herschel-Bulkley model, Eq. (2.5), whereas the Cross model characterized the pseudoplastic fluid. Rheological parameters for Herschel-Bulkley model were determined as: $\tau_{0}^{H}=10 \mathrm{~Pa}, m=0.167 \mathrm{~Pa} \cdot \mathrm{s}^{n}$ and $n=0.535$. The experimental study was carried out by monitoring the axial velocity and the pressure drop along the test section. For the turbulent flow of non-Newtonian fluids, the experimental study showed a decreasing of the friction factor as the $R e$ was increased. Also, it was determined the flow nearby to the centerline, where the turbulence intensity is similar, for both cases, Newtonian and non-Newtonian fluid. However, for the region near to the wall, the non-Newtonian fluid presents a higher turbulence intensity than the Newtonian fluid one. The mean velocity $(\bar{U})$ was used to normalize the centerline velocity $\left(u_{C L}\right)$, the velocity profiles, and the first-order quantities for turbulent and laminar regimes. These results showed agreement with analytical solutions 
and the data available in the literature, however also as Rudman et al. (2004), the author remarked the difficult of set the same rheological properties for numerical simulations according to the experimental data.

A significant result of those experimental studies in straight pipes flow is the presence of asymmetric behavior in the flow of viscoplastic fluid. Rudman et al. (2004) and Peixinho et al. (2005) stated that asymmetric behavior starts when the flow is in transitional regime. This asymmetry was also detected by Escudier and Presti (1996) for thixotropic fluid flow with yield stress. According to the authors, the asymmetry shows the instabilities in the flow during the transitional regime and can be intensified by geometrical imperfections along the flow-loop. Also, the high shear rates during the transitional regime may deform the material until turbulent conditions are established. Nevertheless, this is a matter of ongoing studies to understand this behavior in a deeper way.

\subsection{FLOW OF VISCOPLASTIC FLUIDS IN CONTRACTIONS}

Some numerical studies have been published on the behavior of viscoplastic fluids flowing through a contraction. In the planar case, Coupez et al. (1994) used an augmented Lagrangian method to solve the flow of Bingham fluids. They calculated the yielded zone in the case of a $\beta=8$ contraction with a $45^{\circ}$ contraction angle. Bingham numbers varied between 400 and 20. Isayev and Huang (1993) proposed the study of a viscoelastic plastic medium (a carbon black filled rubber compound) for a $\beta=4$ contraction and expansion with entrance angles of 90,45 , and $15^{\circ}$. They identified the location of the yielded and unyielded regions for different flow rates and pressure drops. As the flow rate increases, the size of the unyielded regions near the contraction decreases, and the pressure along the centerline is higher, and after the contraction, there is a trend to reach similar pressure drops. Gans (1999) presents an analysis for a Bingham fluid flow through contractions, connecting two parallel plates for a variety of contraction ratios ranging $1.1 \leq \beta \leq 4$. The author discusses the presence of a floating core in the downstream flow and defines the core length as a function of the contraction ratio. In this study, it was determined that exists a critical contraction ratio where the relationship between the upstream and downstream plug core is close to 1 . This critical value is set at 1.215 . It was determined that as $\beta$ increases, the core plug at the upstream region starts to move downstream, and the velocity profiles show higher velocities at the centerline.

Concerning axisymmetric conditions, Magnin and Piau (1992) demonstrated, numerically, and experimentally the importance of yield stress on the flow structure and pressure drop both in a $\beta=4$ expansion and contraction. Also, Mitsoulis et al. (1993) has studied the flow of a viscoplastic material numerically through extrusion dies with a contraction ratio of $\beta=10$, using a Herschel-Bulkley model. They showed the extension and shape of the yielded/unyielded regions and how this relation decreases as $B i$ and $\tau_{0}^{B}$ increase. Other studies as Abdali et al. (1992) performed a numerical evaluation of the flow of a Bingham fluid for a $\beta=4$ sudden contraction in planar and axisymmetric dies, comparing the effect for both cases. The yield stress was set between 1 and $2.7 \mathrm{~Pa}$, which lead to Bingham numbers between 1.95 and 264 . The study stated that as the $B i$ value increases, there are lower deformation on the material at the entrance of the contraction, and the plug core of both regions of the contractions growth proportional to $B i$. Also, it is displayed an early restructuring of the downstream plug core, reducing the swell ratio (Ratio between 
the height of the downstream and upstream plug core).

Jay et al. (2002) carried out a numerical simulation of viscoplastic fluid flow through a cylindrical axisymmetric contraction. The influence of the contraction angle and the contraction ratio were performed, using angles between $20^{\circ}$ and $90^{\circ}$ and contraction ratio $2 \leq \beta \leq 8$, also a wide range of $B i$ numbers, from 0 to 100 . The behavior of the streamlines was compared with experimental visualization introducing tracer particles into a gel with rheometrical properties fit by the Herschel-Bulkley model. From this study was concluded that the structure of the flow is highly dependent on yield stress. When $\tau_{0}^{H}$ increases, the size of the plug core and the unyielded regions near to the contraction increase drastically.

An intermediate structure between vortex structure and unyielded regions at the contraction corners was obtained for $B i$ between 0.001 and 0.01 for abrupt contraction (entrance angle of $90^{\circ}$ ). The unyielded regions at the corners disappeared in the case of very small contraction angles, and no vortex structure was detected as $B i$ is increased. Pressure loss also depends on the same parameters. It is almost constant while the $B i$ number is smaller than 0.1. On the other hand, it was showed that for higher values of pressure, the increase in the entrance length is very significant. The length of the conical part of the contraction surface affects the pressure loss, this decreases when the the entrance angle increases, this behavior is more remarkable, especially for high $B i$. This numerical study presented good agreement with Abdali et al. (1992), the plug core region after the contraction increases as $B i$ is increased. This behavior is related to the extrudate swell (die swell); thus, as $B i$ increases, viscoplastic material deformation decreases.

More recently, Fester et al. (2008), Mika (2011) Kfuri et al. (2011) study the energy losses of viscoplastic fluids flow in sudden contractions. Fester et al. (2008) and Mika (2011) developed experimental studies, using different viscoplastic fluids and contraction ratios. Fester et al. (2008) used different solutions of Kaolin, obtaining different rheological parameters: $\tau_{0}^{H}, m$ and $n$. The pressure drop for different sudden contraction ratios $(1 / \beta=0.22,0.5$ and 0.85$)$ were measured. Then, the loss coefficient at the contraction $\left(k_{c o n}\right)$ was calculated using the rheological parameters to calculate the kinetic energy correction factors. Mika (2011) focused a experimental study of ice slurry solutions flow through six different contraction ratios $(1 / \beta=0.5,0.615,0.650,0.769,0.8$ and 0.813$)$. Also, $k_{\text {con }}$ was obtained, in this case were used the Eqs. (2.52)-(2.53), which have a better correlation based on the Reynolds numbers to calculated the kinetic energy correction factor. The data obtained for Mika (2011) compared with the experimental results obtained by Fester et al. (2008) and Turian et al. (1998). It was concluded that for laminar regime $k_{\text {con }}$ depends on the $R e^{\prime}$, but for turbulent regime, due to in turbulent regime the $k_{\text {con }}$ reaches a constant value. However, the correlation factors obtained for each experimental study are different from each other. Apparently, these correlations depends on the properties of the fluid. Table 3.1 summarizes the correlations obtained for each experimental study for a $1 / \beta=0.5$.

On the other hand, Kfuri et al. (2011) performed a numerical study to correlate the loss coefficient for power-law and Bingham fluids flow, for a $\beta=4$ abrupt contraction and at the entrance region, for the entrance flow problem was considered a uniform and fully developed flow configuration before the contraction, axisymmetric geometry for the pipe, and no-slip condition at the walls. For the contraction problem, the same conditions at the entrance flow were considered. For the viscoplastic material a dimensionless yield stress $\left(\tau_{0}^{\prime}\right)$ was set at: $0,0.1,0.3$, and 0.5 . The Reynolds number assumed values of 50, 250 and 1000. In the case of the entrance flow, there was a similar behavior between power-law 
Table 3.1 - Correlations for $k_{\text {con }}$ obtained at laminar and turbulent regimes in different experimental studies for $\beta=2$.

\begin{tabular}{cccc}
\hline Authors & Fluid & Laminar & Turbulent \\
\hline Fester et al. (2008) & Kaolin 5\%, 8\% and 10\% & 288/Re' & - \\
Mika (2011) & Slurry ice-mass fraction: 25\% & $11000 / R e^{\prime 1.26}$ & 0.38 \\
Turian et al. (1998) & Gypsum and Laterite & 900/Re & 1.114 \\
\hline
\end{tabular}

Source: Author (2019).

shear-thinning fluids and viscoplastic materials, thus the correlation of the product of friction factor and Reynolds number $\left(f R e_{M R}\right)$ with $\tau_{0}^{\prime}$, an increase on the dimensionless yield stress induces a decreasing of the $f R e_{M R}$ value, in the same fashion as decreasing the power-law index $(n)$, that means a more shear-thinning curve. The location where the flow achieves its fully developed profile is not a strong function of the rheology. For the Bingham material, as the power-law fluid, the curves which relate $f R e_{M R}$ with the dimensionless position $(z / R)$ for different $\tau_{0}^{\prime}$, converge with the direction of the flow collapsing at a point that determines the length of development from a uniform profile.

Figure 3.1 - Local friction coefficient as a function of the Reynolds number for different dimensionless yield stress. (a) Entrance flow with a uniform profile. (b) 4:1 abrupt contraction.
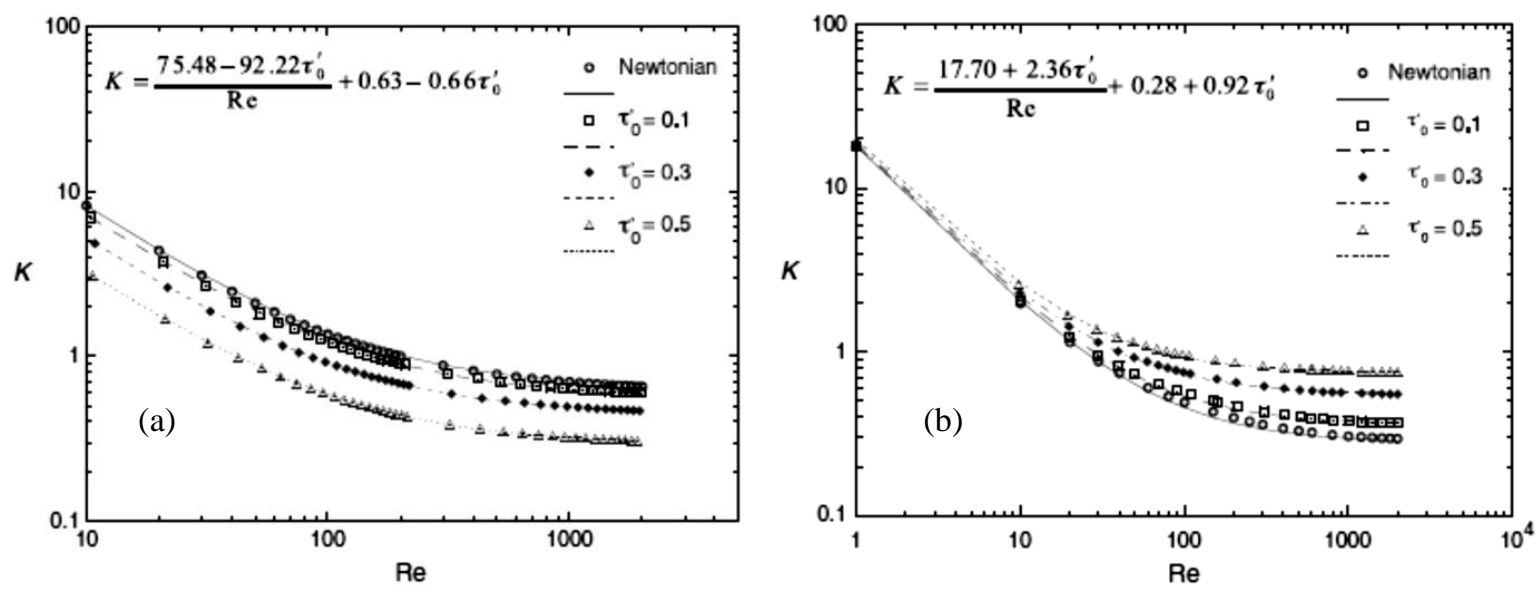

Source: Kfuri et al. (2011).

At the abrupt contraction, Kfuri et al. (2011) found that when the dimensionless yield number for viscoplastic fluids increases, the recirculation size decreases (vortex structures). This is consistent with the results obtained from the numerical study of Jay et al. (2002). And for the local friction coefficient $K$ as a function of the Reynolds number for different values of $\tau_{0}^{\prime}$ showed that for low values of $R e_{M R}$ the different viscoplastic fluids have a very similar behavior, following a linear tendency on a log-log graph. However, as $R e_{M R}$ increases the curves tend to detach from each other with the Newtonian fluid having lower values, see figure 3.1 .

Finally with the compilation of the data from this study, it was possible to find out functions for $K$ for each type of fluid at the entrance $(E N T)$ and for $\beta=4$ abrupt contraction $(A C)$, expressed as follows:

$$
K_{\tau_{0}^{\prime}}^{E N T}=\frac{75.48-9.22 \tau_{0}^{\prime}}{R e}+0.66 \tau_{0}^{\prime}+0.63
$$




$$
K_{\tau_{0}^{\prime}}^{A C}=\frac{17.70-2.36 \tau_{0}^{\prime}}{R e}+0.92 \tau_{0}^{\prime}+0.28
$$

Kfuri et al. (2011) showed that there is a sensitivity of the friction coefficient $(K)$ with the Reynolds number and the yield-stress. For high values of $R e_{M R}, K$ is linear with $n$ for a power-law fluid and linear with $\tau_{0}^{\prime}$ for viscoplastic materials. Instead for low $R e_{M R}$ the power-law behavior is exponential with $n$ and linear in the entrance flow and obeys a logarithmic function in the abrupt contraction. For viscoplastic materials, a linear behavior is found for low $R e_{M R}$ values, for both geometries analyzed in this study. Despite of the equations presented have an excellent fitting, experimental results were remarked as necessary in order to give a better validation for these analyses.

\subsection{VISUALIZATION OF VISCOPLASTIC FLUIDS FLOW IN CONTRACTIONS BY PIV}

Flow in pipes and annular sections with viscoplastic fluids were the first to be studied due to its use in different type of piping.

A relevant study of Hammad et al. (1999) analyzed the laminar flow of a viscoplastic fluid through an abrupt expansion-contraction facility using experimental and numerical studies, and the data were compared with the Newtonian fluid behavior for this geometry. Reynolds numbers between 1.8 a 58.7 were set for the experimental analysis. In the numerical analysis, the viscoplastic fluid was modeled as a Herschel-Bulkley fluid. Both studies were performed through an expansion-contraction system with a ratio of $\beta=2$. This study used a PIV technique of visualization to determine the velocity profile of the plug flow. As it was mentioned in chapter 2, this region is at the center zone of the flow for circular pipes, where there are no velocity gradients for the radial direction, showing a good agreement with the information available for this type of flow.

Ozalp et al. (2007) studied the water flow through an abrupt axisymmetric contraction with a ratio of $4: 1$, using the PIV technique. The aspect ratio was defined as $\beta=D / d$, where $D$ and $d$ are the inlet and outlet diameter of the contraction, respectively. The flow is driven by gravity effects, allowing to generate Reynolds numbers of $223,336,880$, and 1325 . This study determined that local velocities increase with an increase in the Reynolds number. The maximum values for radial an axial velocity were found at the centerline, showing good agreement with numerical results found in the literature.

Finally, Souza-Mendes et al. (2007) studied the internal flow of viscoplastic fluids through ducts with an abrupt axisymmetric expansion followed by an abrupt contraction. The study shows numerically and experimentally the behavior of Carbopol@676 aqueous solutions at different concentrations. The fluid was modeled as the generalized Newtonian fluid, using the following equation to express the shear stress:

$$
\tau=\left[1-\exp \left(-\frac{\eta_{0} \dot{\gamma}}{\tau_{0}}\right)\right]\left(\tau_{0}+m \dot{\gamma}^{n}\right)
$$

where $\tau_{0}$ is the yield stress, $\eta_{0}$ is the viscosity at a low shear rate, $m$ is the consistency coefficient, and $n$ the flow behavior index, see Eq. (2.5). 
The study consider a steady flow at the test zone, and neglect the effects of the inertial forces, assuming Reynolds numbers below 0.1 for the smallest diameter. The experimental observations were made using a laser sheet and a CMOS (Complementary Metal-Oxide-Semiconductor) camera to record and establish a correlation between micron-size spherically particles during the flow. Several radius ratios between the larger $\left(R_{0}\right)$ and the smaller pipe radii $\left(R_{1}\right)$ and the transition length $\left(L_{0}\right)$ are analyzed, in order to determine its effects on the flow behavior. Also, the displacement efficiency is defined as the central region of the flow, where the shear stress is greater than the yield stress. This is expressed as follow:

$$
\Phi=\frac{V_{c y}}{V_{c}}
$$

where $V_{c y}$ is the volume of yielded liquid into the tube cavity, and $V_{c}$ is the total volume of the cavity. Also, a dimensionless shear stress was defined as:

$$
\tau^{*}=\frac{\tau}{\tau_{0}}
$$

This equation is valid for different values of wall shear stress. For dimensionless wall shear stress the relation is defined as $\phi$ :

$$
\tau_{w}^{*}=\phi=\frac{\tau_{w}}{\tau_{0}}
$$

About the rheological parameters, it is observed that displacement efficiency decreases with higher flow behavior indexes $(n)$. The numerical shear stress field obtained is symmetric concerning the vertical plane, as opposed to the behavior observed experimentally. However, this qualitative difference is attributed to the elastic behavior of Carbopol solutions in cases where shear stress is lower than the yield stress.

\subsection{CRITERIA FOR VISUALIZATION OF FLOWS BY PIV TECHNIQUE}

As it was mentioned in the previous sections, the visualization is one of the most crucial factors to meet the goal of the present project. So, it is necessary to analyze some parameters in relevant earlier studies for turbulent flow using the PIV technique.

Westerweel et al. (1996) carried out an experimental study to compare the Digital Particle Image Velocimetry (DPIV) with conventional PIV and LDV for non-trivial unsteady flow, setting some parameters and definitions to future projects. The DPIV method is very reliable when working with small interrogation windows, reducing from the conventional size of $256 \times 256$ to $32 \times 32$, and the accuracy is not affected while Gaussian peak-fit estimator is used as an estimation for correlation, this technique has been implemented into the last generations of PIV systems. In this study Westerweel et al. (1996) ensured a developed turbulent flow, passing the flow through a settling chamber before entering the pipe, generating a laminar flow state at the pipe inlet, afterward, a disturbance was originated from a sudden expansion of about $4 \mathrm{~mm}$ at the pipe inlet. The transition from the inlet flow to a fully-developed condition took place within 40 diameters from the pipe inlet. 
According to Westerweel et al. (1996) and Elghobashi (1994) the ability of the tracer particles to follow the motion on the fluid has to be calculated and this is given by the particle response time:

$$
t_{p}=\frac{\rho_{p}}{\rho} \frac{d_{p}^{2}}{18 \nu}
$$

where $\rho_{p} / \rho$ is the ratio of the density of the tracer particles with respect to the density of the fluid and $d_{p}$ the diameter of the tracer particle. These tracer particles are able to accurately follow the fluid motion when $t_{p}$ is smaller than the Kolmogorov time scale (WESTERWEEL et al., 1996), defined as:

$$
\tau_{k}=\frac{\eta_{k}^{2}}{\nu}
$$

with $\eta_{k} u_{*} / \nu=1.6$, where $u_{*}$ is the friction velocity for turbulent flow of Newtonian fluid and $\eta_{k}$ is the Kolgomorov length scale that is related with the average rate of dissipation of turbulence kinetic energy per unit mass, $\varepsilon$ (STANISLAS et al., 2003).

$$
\eta_{k}=\frac{\nu^{3}}{\varepsilon}
$$

The results obtained from this study showed that DPIV is a technique with the ability to resolving displacement smaller than 0.5-pixel units. The results demonstrated the capability to generate reliable and accurate results with fast interrogation analysis. Hence the results for the turbulent flow statistics demonstrate that the flow conditions for all measurements indeed match closely with LDV data. Also was demonstrated with a comparison of the velocity profiles and the residual profiles that the differences are within the estimated sampling error.

Another relevant study was performed by Doorne and Westerweel (2007). They studied laminar, transitional, and turbulent flow through a pipe using the PIV technique. This experimental study was performed with water as a Newtonian fluid, circulating in a circular cross-section pipe with a diameter of $40 \mathrm{~mm}$. The laser equipment was set to allow the visualization in a three-dimensional configuration. Two cameras were placed at an angle of $45^{\circ}$ to the plane of the laser beam. In order to avoid distortion generated by the surface of the tube, a rectangular test section filled with water surrounds the pipe and two antireflection screens were attached to improve the visualization of the phenomena. The laminar flow study revealed the importance of precise alignment of the light sheet for the calibration plane. A misalignment as small as $0.1 \mathrm{~mm}$ can lead to significant errors due to the misregistration of the displacement of the particle. Proper alignment reduces the noise level almost to $1 \%$ of the maximum streamwise velocity that corresponds to 0.1-pixel units for individual vectors. The measurements from this experimental study allowed to derive several quantities that had been obtained from numerical simulations. The velocity vectors were compared with direct numerical simulation (DNS), and very high accuracy was showed for radial and axial velocity, Eggels et al. (1994). At the same time it was possible to characterized 3D structures of vortices and wall streaks in the flow. 


\subsection{CHAPTER CONCLUSION}

From the literature review, it is noticed that there are several projects related to the experimental approach with the PIV system regarding Newtonian fluid flows performed in laminar, turbulent, and also in transitional flow, validating the analytical solutions and some numerical predictions data. On the other hand, due to the complexity of the non-Newtonian fluids behavior, there are few studies with viscoplastic fluids at laminar and turbulent conditions with the use of the PIV technique to determine the velocity vector fields, streamlines, and the vortex structures behavior, especially in abrupt contractions. Also, the fews visualization studies have been focused on qualitative verifications. Evidencing a quantitative knowledge gap in this area, in which the present study can contribute with experimental data as a source of validation for future experimental projects and numerical simulations, using as viscoplastic fluid different solutions of a commercial gel composed by Carbopol. 


\section{METHODOLOGY}

This chapter describes the steps developed and the next that will be taken to obtain the solution of the proposed study. These steps include, the experimental setup, validation of PIV parameters, experimental performance, data analysis, organization, and interpretation of the results in the form of a scientific document and publish the main results.

\subsection{DESIGN PARAMETERS}

The parameters for the apparatus design have been calculated according to the objectives, fluids and the operational settings required for this experimental study. This section presents a summary of the main parameters to accomplish the objectives of the present project.

From the initial chapter of this document, the objectives can be summarized by two items as follows:

- Determine the pressure losses along the downstream and upstream regions of the contraction for a viscoplastic fluid, in order to determine the pressure loss coefficient as a function of the rheological fluid properties and the Reynolds number based on Malin (1998) criteria.

- Visualize and analyze the factors involved in the dynamics of viscoplastic flow through area contraction, such as plug core region, corner unyielded region, vortex structures generation, and calculation of the mean velocity field for laminar and turbulent regimes.

To accomplish these objectives it was necessary to design an experimental apparatus that allows performing the study according to the required parameters. At the next subsection, there is a brief description of the fluids and the operation parameters for this experimental study.

\subsubsection{Working Fluid}

In the choice of the properly viscoplastic fluid to be used in this work, some considerations must be made, such as refractive index, fluid viscosity, density, chemical compatibility, chemical stability, safety, and price. The most common fluid with viscoplastic properties is a carbopol solutions (STEIN, 2005; PEIXINHO et al., 2005), commonly used in CERNN to represent viscoplasticity.

Stein (2005) carried out a experimental study about the displacement of non-Newtonian fluid in eccentric annular tubes with application in the oil industry, and a relation between the polymer solutions with drilling fluids was found. In this study, it was found that the steady flow curve of a Carbopol@ 676 aqueous solutions (deionized water) from B.F. Goodrich with a weight concentration of $0.09 \%$ wt fitted with the steady flow curve of a viscoplastic drilling fluid. So, the Carbopol solution depicts a similar viscosity behavior of the based-water drilling fluid. 
Rheological tests have been performed at CERNN and the viscoplatic properties of polymer solutions of Carbopol ${ }^{\circledR}$ have been validated, therefore, it was decided to use a commercial gel composed by carboxivinyl polymer, as viscoplastic fluid. The table 4.1 summarizes the main properties of RMC ${ }^{\circ}$ ultrasound gel, chosen as working fluid.

Table 4.1 - Ultrasound commercial gel properties

\begin{tabular}{l|l|l}
\hline General information & Composition & $\begin{array}{l}\text { Carboxivinyl polymer, purified water, } \\
\text { conservatives, thickening agent. } \\
\text { Biodegradable product. } \\
\text { Not registered. }\end{array}$ \\
& $\begin{array}{l}\text { Ecologic information } \\
\text { Toxicological }\end{array}$ & $\begin{array}{l}\text { N.5-7.0 } \\
\text { Crystal clear gel }\end{array}$ \\
\hline Chemical-physical properties & $\mathrm{pH}$ & $950-970 \mathrm{Kg} / \mathrm{m}^{3}$ \\
& Appearance & $44-48 \mathrm{~Pa} \cdot \mathrm{s}$ \\
& Density & $100 \%$ \\
\hline
\end{tabular}

Source: Author, based on manufacturer specifications (2019).

As presented in Tab. 4.1, the $\mathrm{pH}$ of the ultrasound gel is neutral. For that reason, distilled water with a $\mathrm{pH}$ closed to 7.0 was used for each solution. A neutral $\mathrm{pH}$ guarantees a maximum initial viscosity and good stability for the fluid (GIUSEPPE et al., 2015). Different concentrations weight percentage (\%wt) relations were made: $40 \%, 30 \%, 20 \%$, and $10 \%$. Every solution was agitated for a least 12 hours to ensure the homogenization of the fluid, and avoiding absorption of humidity. Then, the recipient was covered, and the fluid was let to rest for 12 hours before starting the experimental tests to remove the air bubble that could be trapped into the solution during the homogenization process.

Color-fixed indicator strip was used to check the $\mathrm{pH}$ during each stage of the solution. The ultrasound gel has high water solubility and neutral $\mathrm{pH}$, so, there were no changes in the $\mathrm{pH}$ value measured during the solutions preparation. Section 4.2 describes in detail the rheological characterization of the different gel solutions, performed at the CERNN laboratory of rheology.

\subsection{VISCOPLASTIC FLUID CHARACTERIZATION}

This section discusses the characterization of the rheological behavior for the fluids, also the refraction index and its comparison with other materials.

\subsubsection{Rheological Behavior}

As pointed out in section 4.1, for the current study, an ultrasound gel is used in different weight percent concentrations of 40,30,20, and 10\%wt. As presented in Tab. 4.1, the gel is composed by carboxivinyl polymer (Carbopol@ 940). This gel has the same rheological characterization of a viscoplastic fluid as the Carbopol solutions used in different experimental studies (TAGHAVI et al., 2012; POUMAERE et 
al., 2014; GIUSEPPE et al., 2015). The figure 4.1 shows the viscosity and shear stress behavior of the ultrasound gel solutions at different shear rates, fitted by a Herschel Bulkley model.

Figure 4.1 - Steady flow curve (a) and viscosity (b) behavior obtained for each fluid. Rheological data were obtained with a HAAKE-MARS III.
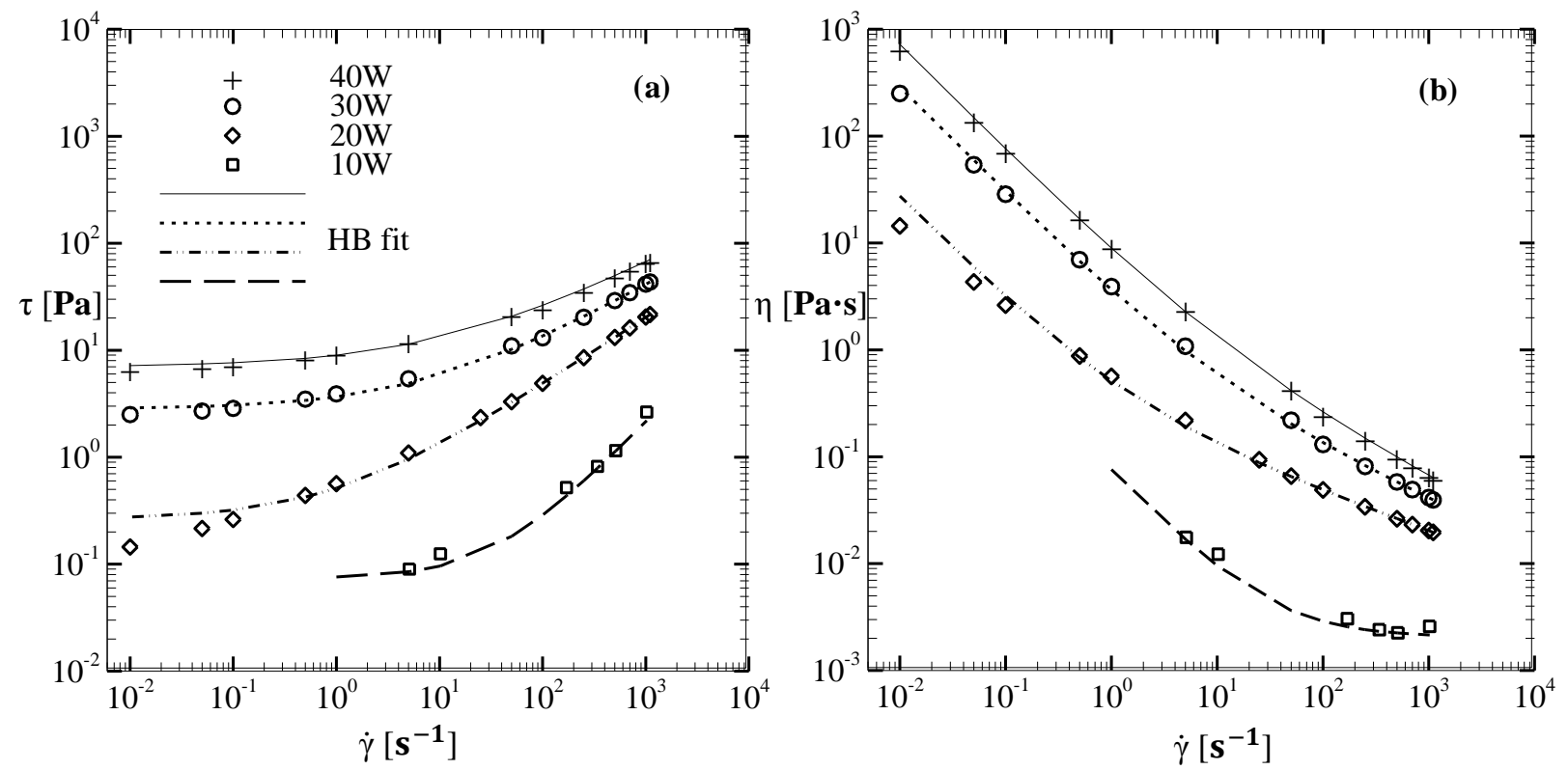

Source: Author (2019).

The rheological tests were performed in a HAAKE-MARS III (Modular Advanced Rheometer system). The steady flow curve for each solution was obtained using different rheometer geometries: cross-hatched parallel plates (CH-PP), cone-plane (CP), and concentric smooth cylinders (CC). In order to avoid wall slip effects at low shear rates, cross-hatched geometry was used for shear rates between 0.01 and 5 $\mathrm{s}^{-1}$. Shear rates between 5 and $1100 \mathrm{~s}^{-1}$ were performed with concentric cylinder geometry, avoiding wrong measurements at high shear rates that can appear due to fluid loss by the cross-hatched geometry (FERNANDES et al., 2019). Cone-plane geometry was used for the lowest weight concentration of ultrasonic gel; this geometry allows an even shear field for aqueous solutions that is not possible to impose with other geometries. Table 4.2 summarized all the parameters set to perform the rheological tests for the ultrasound gel solutions.

Rheological tests were performed at temperature of $25^{\circ} \mathrm{C}$, in order to represent the same conditions set on the laboratory. The steady flow curves were fitted by the Herschel-Bulkley model for each solution, as showed in Fig. 4.1. As the weight percentage of ultrasound gel increases, higher yield stress, and consistency coefficient are obtained, that means a more viscous fluid. On the contrary, a decreasing in the shear-thinning behavior of the fluid is depicted when $n$ has low values, as it is shown for the fluids with the lowest concentration of $30 \%$ and $40 \%$ wt.

For the lowest concentrations of ultrasound fluid, and the viscosity decreases quickly for high shear rates. For $10 \mathrm{~W}$ solution, Fig. 4.1 depicts a constant behavior after a shear rate of $100 \mathrm{~s}^{-1}$ is reached. At this point, the behavior of the fluid is similar to a Newtonian fluid. This behavior can be predicted from the rheological parameters obtained by the Herschel-Bulkley (HB) model fitting, due to $n$ reaches a value 
Table 4.2 - Parameters set for the different geometry configurations for each solution rheological test at HAAKE-MARS III.

\begin{tabular}{l|l|l|l|c}
\hline Concentration & Geometry & Gap $[\mathrm{mm}]$ & Shear rates $\left[\mathbf{s}^{-1}\right]$ & Temperature $\left[{ }^{\mathbf{0}} \mathbf{C}\right]$ \\
\hline $40 \% \mathrm{wt}$ & CC & 0.051 & $1-1100$ & \\
& CH-PP & 1 & $0.01-50$ & \\
$30 \% \mathrm{wt}$ & CC & 0.051 & $1-1100$ & \\
& CH-PP & 1 & $0.01-50$ & 25 \\
$20 \% \mathrm{wt}$ & CC & 0.051 & $1-1100$ & \\
& CP & 0.105 & $0.01-25$ & \\
\hline $10 \% \mathrm{wt}$ & CC & 0.051 & $1-1100$ & \\
\hline
\end{tabular}

Source: Author (2019).

near to the unity, and $\tau_{0}^{H}$ is a very low value. So, at high shear deformations, the $10 \mathrm{~W}$ fluid shows a shear-thinning behavior very quickly, and a Newtonian fluid like behavior is reached.

Table 4.3 summarized the parameters obtained after fitted data to the HB model, such as the yield stress $\left(\tau_{0}^{H}\right)$, the fluid consistency coefficient $(m)$, the flow behavior index $(n)$, and the dimensionless parameter $H e$. In section 2.2, it was defined $H e_{H B}$ as the relationship between yield and viscous stress for viscoplastic fluid. This relation allows predicting the Reynolds number in the transition from laminar to a turbulent regime with reasonable confidence. This critical Reynolds number in the transition increases with the $H e$, according to the correlations of Malin (1998).

For $10 \mathrm{~W}$ solution, there is not defined a $H e_{H B}$ value, thus, low values for $\tau_{0}^{H}$ and $n \approx 1$ yields to a greater $H e_{H B}$ value than the other solutions. Nevertheless, based on theoretical information for these solutions, $H e_{H B}$ reaches values $\approx 0$, similar to the correlations for Bingham fluids (MALIN, 1998). For solutions $30 \mathrm{~W}$ and $40 \mathrm{~W}$, it is necessary greater mean velocities to reach the transitional and turbulent regime, due to these solutions have a greater $H e_{H B}$ value. So, Higher entrance pressures are required for flowing the more viscous solutions, that display greater yield stress values.

Table 4.3 - Herschel-Bulkley parameters and Hedstrom number calculated for ultrasound gel solutions.

\begin{tabular}{c|c|c|c|c}
\hline Concentration & $\tau_{0}^{H}[\mathbf{P a}]$ & $m\left[\mathbf{P a ~ s}^{n}\right]$ & $n$ & $H e_{H B}$ \\
\hline $40 \%$ wt $(40 \mathrm{~W})$ & 6.994 & 1.976 & 0.495 & 16.402 \\
30\%wt (30W) & 2.818 & 0.847 & 0.553 & 18.706 \\
20\%wt (20W) & 0.262 & 0.251 & 0.634 & 2.938 \\
$10 \% w t(10 \mathrm{~W})$ & 0.074 & 0.0023 & 0.986 & $\approx 0$ \\
\hline
\end{tabular}

Source: Author (2019).

\subsubsection{Refraction Index}

Concerning to the PIV technique, an important property to allow the visualization of the fluid flow is the refraction index. The success of a good data acquisition depends on the refractive properties of the fluid and the visualization region. The refraction index is defined as the relation between the speed of 
light in a vacuum $(c)$ and the speed of light in the fluid $\left(c_{f}\right)$. This relation is given by:

$$
n_{r e f}=\frac{c}{c_{f}}
$$

where $c \simeq 3 \times 10^{8} \mathrm{~m} / \mathrm{s}$. Under normal conditions, the speed of light into the fluid is lower than in a vacuum; thus, a fluid refraction index is greater than unity $\left(n_{r e f}>1\right)$. As it was mentioned, most visualization experimental studies use transparent fluids and materials at the test region. Table 4.4 shows refractive properties of common materials.

Table 4.4 - Refractive properties of some materials and fluids for test regions in visualization studies.

\begin{tabular}{lll}
\hline Material/fluids & $\mathbf{n}_{\text {ref }}$ & Description \\
\hline Borosilicate glass & $1.47-1.49$ & Plates, sheets, bars, tubes and special forms \\
Acrylic & 1.49 & Plates, tubes and bars \\
Water & 1.33 & Fluid \\
Glycerin & $1.33-1.47$ & Fluid \\
Ultrasound gel solution & $1.4-1.5$ & Gel or fluid \\
\hline
\end{tabular}

Source: Retrieved from Dynamics (2015) and Raffel et al. (2018).

For the study of fluid flows, the curvature of the pipeline affects the flow visualization due to the refraction index. Westerweel et al. (1996) showed that these effects could be reduced using a visualization test water-filled box. Souza-Mendes et al. (2007) used a visualization box to visualize the viscoplastic fluid flow through a contraction-expansion device. This visualization box was filled with the same working fluid.

Figure 4.2 - Different set of refractions index at the visualization box: (a) optical distortions caused by the curvature of the circular tube. (b) box filled with the same working fluid. (c) equal refraction indices for the circular tube and the working fluid.
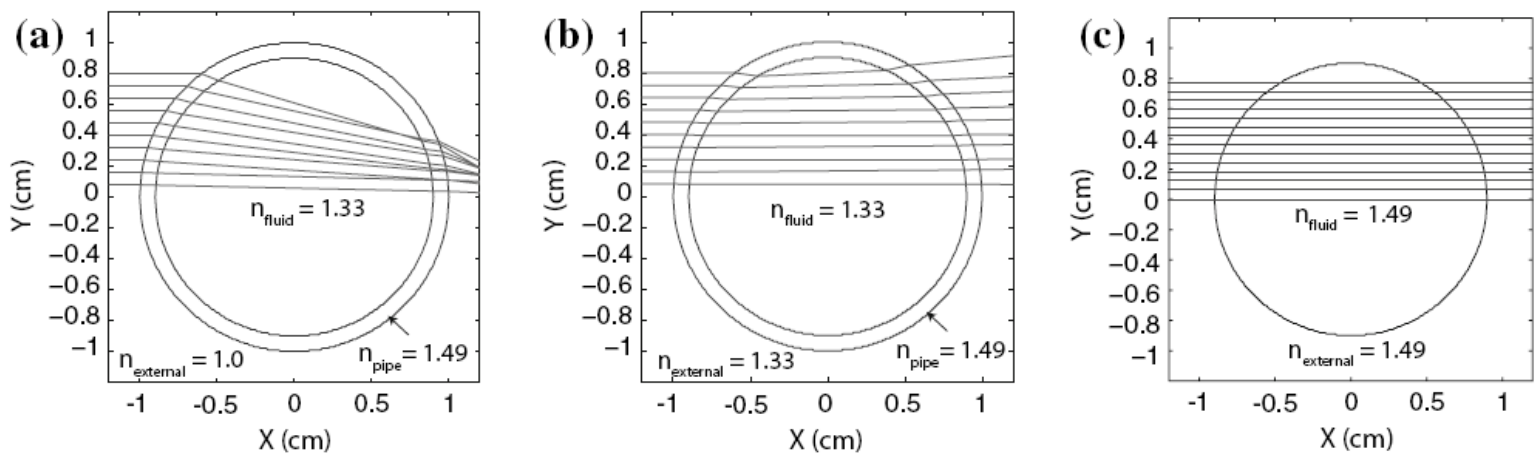

Source: Adapted from Raffel et al. (2018).

Figure 4.2 depicts the effect of the fluid, pipe, and external material $n_{r e f}$ on the optical distortions caused by the wall curvature. Figure $4.2 \mathrm{c}$ presents that the distortion effects can be corrected using materials with the same refraction index. Then, for the present experimental project, a solid piece visualization box made of acrylic is used, as the ultrasound gel solution and the acrylic have similar refraction indices, image distortions are avoided. 


\subsection{EXPERIMENTAL SETUP}

The first stage of this project was the experimental design and construction of the experimental setup, developed the following parameters to achieve the main purpose of this work. After the validation of these parameters, a hydraulic circuit was planned and designed for a contraction ratio of $\beta=1.85$. The structure is supported by a metal structure placed at Research Center of Rheology and Non-Newtonian Fluids (CERNN) of the Federal University of Technology-Parana (UTFPR). Other components as hydraulic, control, and visualization systems complement the experimental setup, and they are presented to have a better understanding of the apparatus operation and how the visualization data are taken and processed.

\subsubsection{Hydraulic System}

The hydraulic circuit was designed with a visualization area composed of straight and cylindrical pipes. Figure 4.3 presents a scheme of the hydraulic system, where the flow-loop system is composed of a reservoir tank ( $i$ ) with a capacity of 100 liters (L), and a paddle system to mix the fluid as required. The fluid is driven by a helical rotor NEMO-pump (positive-displacement pump) (ii) through a circuit made by circular acrylic tubes and PVC (Polyvinyl Chloride) pipes. The flow rate is controlled by a frequency inverter (iii) with the assistance of a mass flow meter (iv). In this context, pump rotation is controlled by the frequency inverter until the desired flow is detected by the flow meter. Other parameters can be measured by the flow meter as density and temperature.

Before the contraction, in the upstream region, the pipe has an internal diameter $(D)$ of $25.9 \mathrm{~mm}$. Along this section, the pressure is measured at three different points with differential pressure transmitters (v), valves are placed at each pressure point to select which pressure points are compared and these facilitate the connection of the transmitters with the hydraulic facility. After the fluid passes through the test section (vi), the velocity field is determined using the PIV technique, and the images obtained are processed by Dynamic Studio software. The test section was designed based on Doorne and Westerweel (2007) study. The authors were concerned about a design that allows the alignment of a laser beam with the camera plane. Also, the test section was designed to avoid oscillations and fluctuations that can compromise resolution and image quality.

At the downstream region of the contraction, the internal diameter $(d)$ is $14 \mathrm{~mm}$. In this section of the flow-loop, the pressure is measured at two points with a differential pressure transmitter.

The data obtained by the differential pressure transmitters and the mass flow meter are processed and stored by a routine developed in a LabVIEW ${ }^{\mathrm{TM}}$ platform, while the images captured by the camera are stored in the camera buffer and consequently are exported to the project data file. Measured parameters are displayed as temperature, density $(\rho)$, pressure drop $(\Delta P)$, friction coefficient $(f)$, and the friction wall velocity $\left(u_{w}\right)$, see Appendix.

\subsubsection{Measuring and Control Systems}

The measuring and control systems are made up of various devices to monitor the parameters and the physical properties of the fluid. The rotation of the helical pump (NETZSCH NM038BY01L06B/2.2 kW) is controlled by a frequency inverter WEG CFW08, connected to a controlling computer, where a routine 
Figure 4.3 - Experimental apparatus: hydraulic system for tests performing.

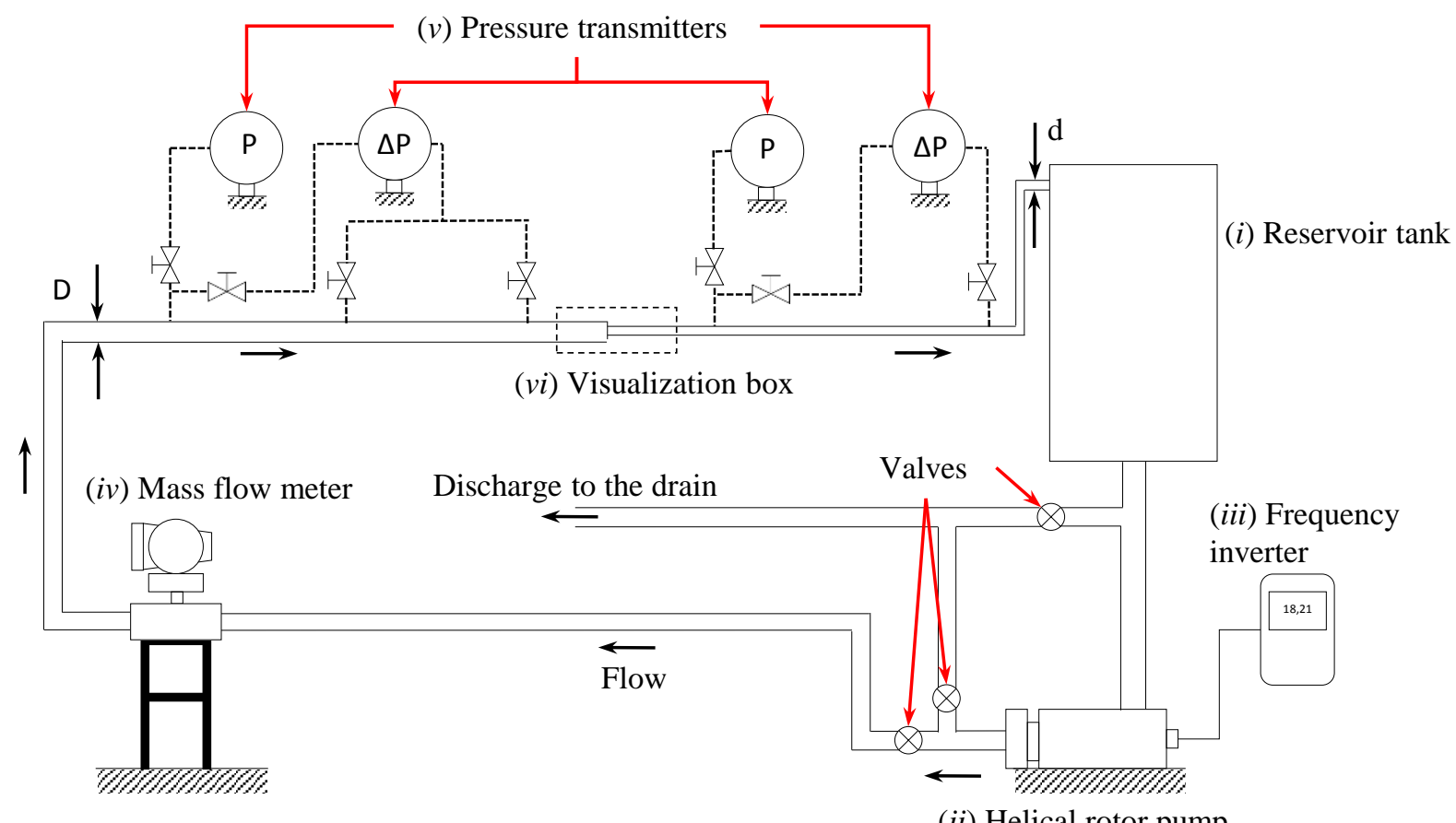

Source: Author (2019).

is used to regulate the volumetric flow rate. As mentioned, the flow rate is measured by a mass flow meter Micro-Motion 2700, that uses the Coriolis flow measuring principle with an oscillating curved tube to obtain the mass flow rate, volumetric flow rate, density, viscosity, and temperature, with an operational range between 3.40 and $68.04 \mathrm{~kg} / \mathrm{min}$.

The flow-loop was designed to measure the pressure at points with distances between $0.3 \mathrm{~m}$ and $1 \mathrm{~m}$, with an accuracy estimated at $0.1 \%$ for span range $(0-62$ mbar $)$ and a total error of $0.4 \%$ for the full range of measurement $(0-138 \mathrm{bar})$. The pressure transmitters are connected to a pressure tappings of 4 $\mathrm{mm}$ internal diameter, and these tappings are connected to cylindrical tubings that are filled with deionized water. The transmitters send the digital signal to a data acquisition National Instruments Fieldbus board PCI/FBUS/2.

The entrance length $\left(L_{e}\right)$ was calculated to guarantee a fully developed flow at the test section. The $L_{e}$ is considered from the pump output until the visualization box, which is the region where is needed a fully developed flow. According to Froishteter and Vinogradov (1980), the most suitable value of the $L_{e}$ for Herchel-Bulkley fluid is given by Eq. (2.37).

In subsection 2.2.2, the equation (2.35) shows that the $R e_{g}$ has a dependence of parameters $n$ and $m$; so, for each $R e_{g}$, it is necessary to calculate $L_{e}$. As the index $n$ increases, the $L_{e}$ value decreases for a constant coefficient $m$. An increasing of $n$ means a more viscous fluid. Table 4.5 shows the $L_{e}$ values calculated for the two laminar $R e_{g}$ at the entrance diameter $D$, that will set in this study. A Reynolds number at the entrance of the test section of $R e_{D_{g}}=1500$ represents a critical value for a solution of $30 \%$ wt ultrasound gel; it is required $8.05 \mathrm{~m}$ to get a fully developed flow. According to the calculations, 
the hydraulic circuit was designed with a $L_{e}=8.3 \mathrm{~m}$ and a total length until the discharge point into the fluid container of $12.5 \mathrm{~m}$. For a concentration of $40 \% \mathrm{wt}$ just low laminar Reynolds number are achieved, with a maximum value of $R e_{D_{g}} \approx 100$; for that reason the $L_{e}$ critical values are calculated for the rest of the viscoplastic solution.

Table $4.5-L e$ values required for a fully developed laminar flow at $R e_{D g}=1000$ and 1500 .

\begin{tabular}{ccc}
\hline & \multicolumn{2}{c}{$L_{e}[\mathrm{~m}]$} \\
\hline & $R e_{D_{g}}=1000$ & $R e_{D_{g}}=1500$ \\
$\mathbf{3 0 \%} \mathbf{w t}$ & 6.70 & 8.05 \\
$\mathbf{2 0 \%} \mathbf{w t}$ & 5.47 & 7.16 \\
$\mathbf{1 0 \%} \mathbf{w t}$ & 1.11 & 4.66 \\
\hline \multicolumn{3}{c}{ Source: Author (2019). }
\end{tabular}

So, it is guaranteed a fully developed flow at the laminar regime for the solutions selected to carry out the experimental study. Another essential variable is the temperature of the fluid, due to continuous pumping inside the hydraulic circuit, the fluid can be heated, and it is necessary to monitor the temperature. The Coriolis flow meter allows measuring the temperature of the fluid at the pump outlet section. All the experimental study was performed with a room temperature of $25^{\circ} \mathrm{C}$, in order to assure the same temperature conditions of the rheological tests performed at LabRheo for each solution.

\subsection{TEST SECTION}

As mentioned in the last section, it was used a solid piece of acrylic with an inner contraction as a visualization box. This design avoids keeping the box filled with water or another fluid to acquire images, and it is not necessary to use rubber rings and other sealed material to avoid leaking. The acrylic piece was designed with $300 \mathrm{~mm}$ length, $45 \mathrm{~mm}$ height, and $45 \mathrm{~mm}$ width. These dimensions were chosen to facilitate the visualization of fluid and good stability of the test section. At the entrance of the test section, there is an acrylic tube with an inner diameter of $25.9 \mathrm{~mm}$ and an external diameter of $32 \mathrm{~mm}$. At the exit of the test section, there is a hole with a diameter of $20 \mathrm{~mm}$; this allows connecting the small tube diameter of the flow-loop with an inner diameter of $14 \mathrm{~mm}$. Figure 4.4 shows the dimensions of the solid acrylic box.

The solid acrylic box allows excellent visualization of the seeding particles and low light scattered by the walls at the downstream and upstream region of the contraction. According to Tab. 4.4 the refraction index between the acrylic and the ultrasound solutions is not equal, but close. This difference in the refraction index can lead to slight distortions of the captured image. The image distortions were corrected with Dynamic Studio software features, as exposed further in sections 4.6 and 4.7. 
Figure 4.4 - Schema of the contraction test section with dimensions expressed in millimeters $(\mathrm{mm})$. The test section is made of a solid acrylic piece, designed to fit with the flow-loop pipes at entrance and exit sections.

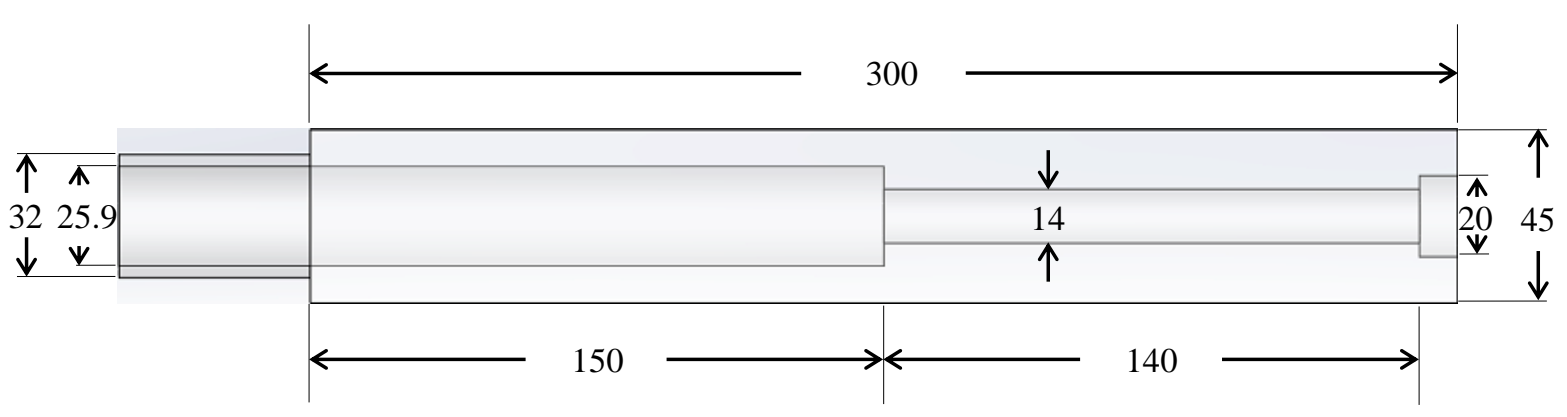

Source: Author (2019).

\subsection{PIV SYSTEM}

As it was described in section 2.3, the PIV system is composed by the integration of components, where the most relevant are:

- Pulsed laser Nd:YAG with Dual Power 100-500 cavities from Litron Laser Ltd. This laser system has a wavelength $(\lambda)$ of $532 \mathrm{~nm}$ (green light), maximum power of $60 \mathrm{~mJ}$ per pulse, and a maximum pulse rate of $100 \mathrm{~Hz}$.

- CMOS Nanosense MkIII camera, with a maximum resolution of $2352 \times 1728$ pixels, an storage capacity of $8136 \mathrm{MB}$, a maximum frequency data acquisition of $100 \mathrm{~Hz}$, and a Micro-Nikkor AF (Nikon) $60 \mathrm{~mm}$ objective lens.

- Timer box 9080 N0771 from Dantec Dynamics to synchronize the laser emission with the camera image acquisition.

- Dynamic Studio software for the image capturing and post-processing.

The camera and the laser were aligned in angle of $90^{\circ}$ to obtain a perpendicular position between the laser beam and the visualization plane. An optical table is used for positioning and guarantees the stability of the laser system and to allow a suitable maneuver of the equipment; the vertical position of the laser beam is controlled by an elevating table mounted over an anodized aluminum structure as shown in the figure 4.5.

\subsubsection{Seeding Particles}

The PIV technique is based on the measuring of the average displacement of particles added to the fluid, for this reason, it is crucial a suitable selection of particles, allow a good correlation with the fluid velocity field. The choice of tracer particles depends on many parameters that are described below. 
Figure 4.5 - Laser and visualization system, the plane of the laser beam and the visualization plane of the camera are set in a perpendicular configuration. Red lines show the perpendicular configuration.

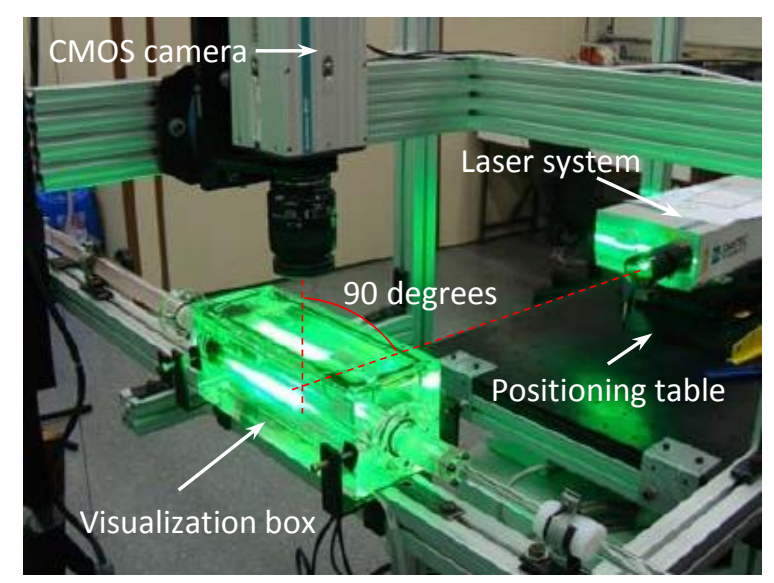

Source: Adapted from Palacios (2011).

\subsubsection{Properties}

The particles should be small enough to follow the flow without interfering in its physical and flow properties. Although the effect due to the difference of the fluid and particles density can be neglected, there is an induced gravitational velocity $\left(U_{g}\right)$, known as settling velocity, which is derived from the Stokes law, to study the acceleration of the particles. The velocity $U_{g}$ is expressed as a relative velocity; this results from the difference between the fluid density and the density of the particle. The following equation expresses this relation, assuming perfect spherical particles (WESTERWEEL et al., 1996; RAFFEL et al., 2018):

$$
U_{g}=d_{p}^{2} \frac{\left(\rho_{p}-\rho\right)}{18 \mu} g
$$

where $g$ is the gravity acceleration, $d_{p}$ is the particle diameter, $\rho_{p}$ is the density of the particles and $\rho$ and $\mu$ are the density and the apparent viscosity of the fluid. The table 4.6 shows the average diameter of different types of particles used as tracers for fluid flow.

Table 4.6 - Different tracer particles materials for fluids flow, retrieved from Raffel et al. (2018).

\begin{tabular}{lll}
\hline Type & Material & Average diameter $[\mu \mathbf{m}]$ \\
\hline Solid & Polystyrene & $10-100$ \\
& Aluminum flakes & $2-7$ \\
& Hollow glass spheres (HGS) & $10-100$ \\
& Granules for coating & $10-500$ \\
& Polyamida (PSP) & $5-100$ \\
& Polymethyl methacrylate (PMMA) & $5-50$ \\
Liquid & Special oils & $50-500$ \\
Gas & Oxygen bubbles & $50-1000$ \\
\hline
\end{tabular}

Source: Retrieved from Dynamics (2015) and Raffel et al. (2018). 
From which equation (4.2), another expression is derived. It is an expression for the delay velocity $\left(U_{s}\right)$, this is the relative velocity between the particle and the instantaneous fluid velocity with acceleration (a), given by (RAFFEL et al., 2018):

$$
U_{s}=U-U_{p}=d_{p}^{2} \frac{\left(\rho_{p}-\rho\right)}{18 \mu} a
$$

or in a differential expression as:

$$
\frac{d U_{p}}{d t}=\frac{U-U_{p}}{d_{p}^{2} \frac{\left(\rho_{p}-\rho\right)}{18 \mu}}
$$

where $U_{p}$ and $\bar{U}$ are the velocities for the particle and the fluid, respectively. The solution for Eq. (4.4) is found assuming that the density for the particle is much greater than the fluid density, $\rho_{p} \gg \rho$. The expression for $U_{p}$ shows an exponential behavior (RAFFEL et al., 2018):

$$
U_{p}(t)=\bar{U}\left[1-\exp \left(-\frac{t}{\lambda_{s}}\right)\right]
$$

where $\lambda_{s}$ is the relaxation time defined by (WESTERWEEL et al., 1996; RAFFEL et al., 2018):

$$
\lambda_{s}=d_{p}^{2} \frac{\rho_{p}}{18 \mu}=d_{p}^{2} \frac{\rho_{p}}{18 \nu \rho}
$$

$\nu$ is the kinematic viscosity of the fluid, $\lambda_{s}$ is the capability of the particles to follow the flow and, when its value is lower than the relation $D / \bar{U}$ (time scale of flow, see section 4.6.2), the particles suitably follow the dynamics of the fluid.

From equation (4.6) it is possible to conclude that it is desired to use small particle diameters to get a short relaxation time. Nevertheless, it is necessary to consider that the diameter particle affects the light reflection captured by the camera. Table 4.6 shows range of diameters for the different particles used with the PIV technique.

At the first stage of this project, the methodology for the PIV technique was evaluated with water as a Newtonian fluid, using hollow glass spheres as tracer particles (see Appendix A), which are more critical conditions than those for viscoplastic fluids due to the low viscosity of the water. The hollow glass particles refract the light induced by the laser system, which is emitted at $532 \mathrm{~nm}$. The wavelength is the same emitted by the laser system. The light is filtered by a polarizer, which allows setting the quantity of light that is captured by the camera. These particles have a density of $\rho_{p}=1.15 \mathrm{~g} / \mathrm{cm}^{3}$ and an average diameter of $d_{p}=10 \mu \mathrm{m}$. Applying the Eq. (4.3), these values lead to a settling velocity of $U_{s}=6.14 \times 10^{-7} \mathrm{~m} / \mathrm{s}$, which is a small magnitude value compared with the values for $\bar{U}$, in consequence the particles can reach the flow.

Additionally, the $\lambda_{s}$ value for the hollow glass particles is $6.38 \times 10^{-6} \mathrm{~s}$; it is a lower value than the time scale calculated for water at the critical Reynolds number of 24600, this is the highest Reynolds used for the validation stage of this project. The time scale calculated for this condition was $0.027 \mathrm{~s}$. In section 4.6.2 are summarized the equations that define the integral time scale.

Finally, lower time scale values mean that the relaxation time uncertainty can be neglected, and the settling velocity shows that diameter particles around $10 \mu \mathrm{m}$ are suitable to reach the high Reynolds number, and consequently the Reynolds number proposed in this project. 


\subsubsection{Optical filter}

The HGS particles are most commonly used in PIV applications, for this type of seeding particles a polarizer filter is used. This optical filter lets light waves of a specific polarization pass through while blocking light waves from others. This type of filter allows removing unwanted reflections from nonmetallic surfaces such as water, glass, and acrylic. Also, it enables colors to become more saturated and clearer, with better contrast. As it was mentioned in the first part of this project, the validation of the PIV parameters was carried out with a Newtonian fluid, using HGS as seeding particles and a polarizer filter. A CIR-PL Slim of $62 \mathrm{~mm}$ filter from HOYA filters was used, this is a linear filter, which maintains the same axes of polarization with varying angles of incidence.

Figure 4.6 - Optical filters used for CMOS camera: (a) polarizer filter, commonly used for glass and polymer particles. (b) orange sharp cut filter for polymer particles coated by rodamine-B.
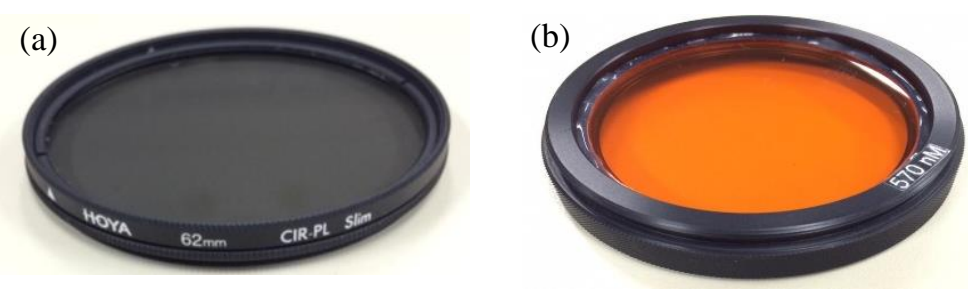

Source: Author (2019).

The final stages of the project were performed with polyamide particles covered by rhodamine-B, which is a colored substance used as tracer dye. The absorption and emission of the rhodamine- $\mathrm{B}$ are shown in table 4.7. In order to get a good correlation of the images acquired, an orange sharp cut filter is used. This is a long-pass filter that cuts off the wavelength light shorter than $570 \mathrm{~nm}$ as much as possible, while transmitting longer wavelength than this value, usually until $640 \mathrm{~nm}$, with a transmittance of $95 \%$. Then, it is possible to block the light scattering by acrylic and glass pieces with this filter and the light properties of the rhodamine-B. It is expected that the noise can be reduced, and a better correlation near the pipe wall may be obtained.

Table 4.7 - Typical range values for absorption and emission spectra of rodamine-B in water solutions. Data taken from: Zhang et al. (2014), Liu and Lu (2015).

\begin{tabular}{lccc}
\hline & Lower limit & Upper limit & Optimal value \\
\hline Absorption wavelength [nm] & 450 & 590 & 554 \\
Emission wavelength [nm] & 550 & 650 & 580 \\
\hline
\end{tabular}

Source: Author (2019).

Figure 4.7 compares the images obtained from a Newtonian fluid flow at $R e=5300$ for different seeding particles: HGS and rhodamine-B. Due to the sharp cut filter blocks the light with a wavelength lower than $570 \mathrm{~nm}$, the camera only can capture the light emitted by the particles coated by rhodamine, blocking the light scattering by the tube walls and the refracted by some worn regions at the inner surface. 
Figure 4.7 - Comparison of images obtained from the light scattered by: (a) HGS particles and (b) polyamida particles coated by rodamine-B. The images were taken from a Newtonian fluid flow.
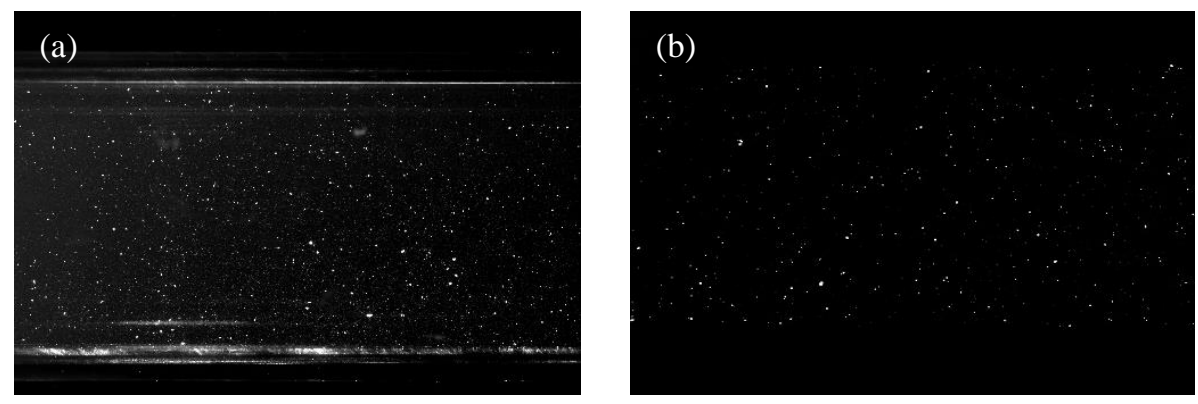

Source: Author (2019).

\subsubsection{Light scattering behavior}

The light contrast of the PIV recordings is directly proportional to the scattered light power, and it is possible to increase the image intensity using the properly scattering particles, instead of increasing the laser power. The light scattered by small particles is a function of (RAFFEL et al., 2018):

- Ratio of the refractive index of the particles and the surrounding medium.

- Size of the particles.

- Shape of the particles.

- Orientation of the light reflected and the laser beam plane.

For particles larger than the wavelength, the light suffers a direct reflection. In this case, the signal captured by the camera is proportional to the square of the particle diameter. For spherical particles with diameter, $d_{p}$, large than the wavelength of the incident light $\lambda$, Mie's scattering theory is applied (RAFFEL et al., 2018). This theory states that the larger is the particle, the more light is reflected (RAFFEL et al., 2018). This effect is presented in figure 4.8 , where are shown the different polar distribution of the light scattered in the logarithm scale, for different particle diameters in water. The refraction index for the glass particles is 1.52 and 1.33 for water. The light wavelength is $532 \mathrm{~nm}$. The intensity for neighboring circles differs by a factor of 100 . The Mie scattering can be characterized by the normalized diameter, $q$, defined by:

$$
q=\frac{\pi d_{p}}{\lambda}
$$

If $q$ is larger than unity, approximately $q$ local maxima appear in the angular distribution over the range from $0^{\circ}$ to $180^{\circ}$.

According to Raffel et al. (2018), as the scattered light is spread in all directions, the higher intensity is obtained for $180^{\circ}$. Then, the light that is imaged by the camera is not only due to direct illumination; it is also due to fractions of light, which have been scattered by more than one particle. In the case of heavily seeded flows, the intensity of the individual particle images is increased for $180^{\circ}$, then, recording at $90^{\circ}$ it is most often used to regulate the light scattering. 
Figure 4.8 - Light scattering by glass particles in water, for different diameters: (a) $1 \mu \mathrm{m}$. (b) $10 \mu \mathrm{m}$. (c) $30 \mu \mathrm{m}$.
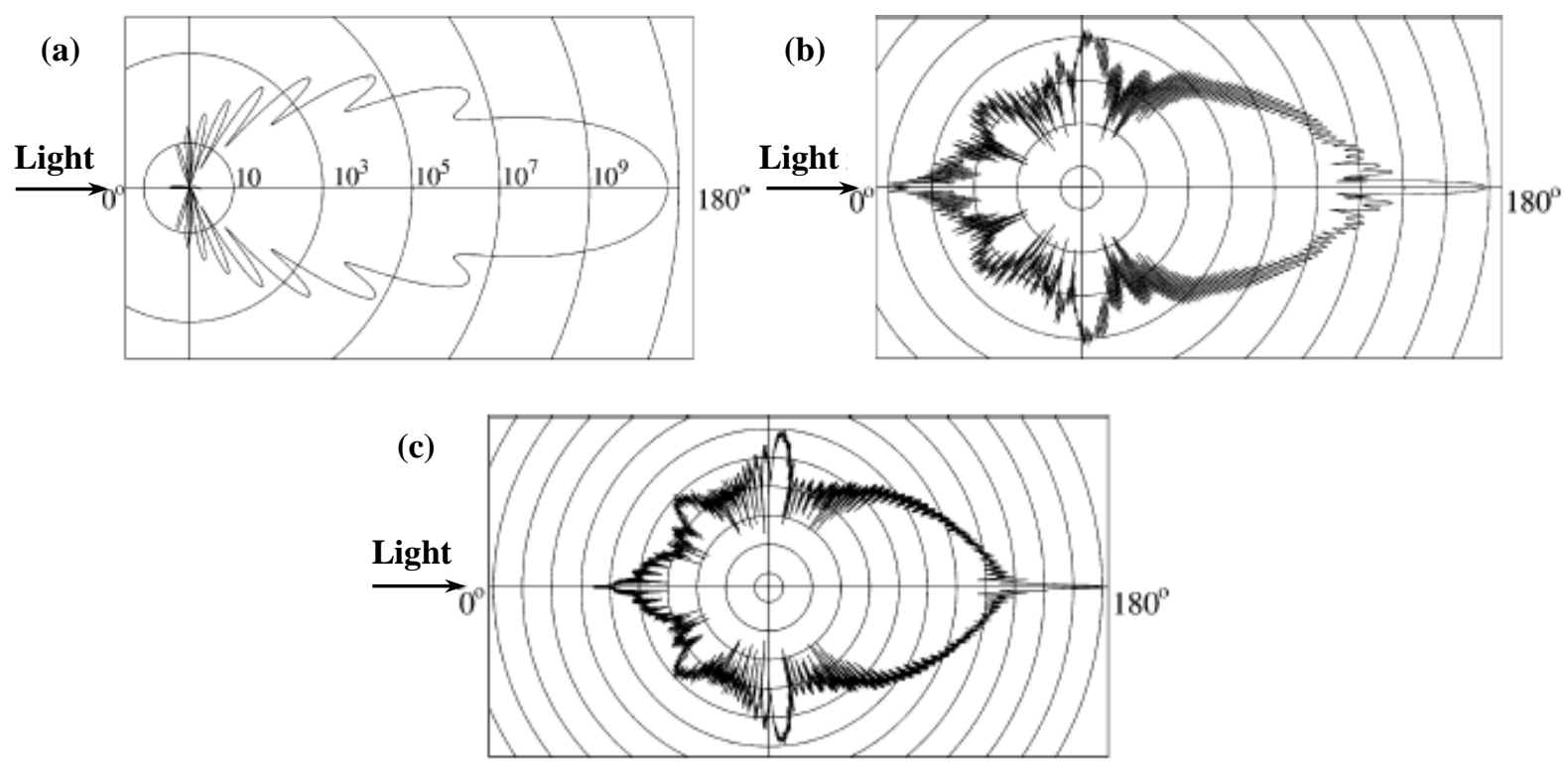

Source: Raffel et al. (2018).

Other implications from the Mie diagrams, applied in this project, is that not only larger particles can be used to increase the scattering efficiency but also a larger density of particles value (number of particles per interrogation area). However, this is limited by possible background noise, and therefore, the noise on the recording is increased significantly. In order to avoid this noise, the correlation of the pair of images is tested, as explained in the following sections.

\subsubsection{Image Recording}

The function of the camera in the PIV system is essential to reach a good correlation. As it was mentioned in section 2.3, there are many types of cameras. The most common and used for PIV applications are CCD and CMOS cameras; these cameras use a kind of sensor known as solid-state sensors. CMOS sensors are suitable to get a high-quality image, but the CCD sensor is widespread used. However, the fast development of the chip technology in the last century allowed the manufacturing of CMOS sensors with an improved signal-to-noise ratio and resolution. In this experimental project a CMOS camera sensor is used, as the best option for high-speed PIV.

A CMOS is an electronic sensor that can convert light (photons) into electric charges (electrons). These sensors are a rectangular array of individual CMOS. An individual CMOS element in the sensor is called pixel, and its size is generally of the order of $10 \mu \mathrm{m} \times 10 \mu \mathrm{m}$, or 100 pixels $/ \mathrm{mm}$. The main advantage of CMOS sensor compared to other techniques like photogates and phototransistors is their high sensitivity and relatively low noise. Compare with CCD pixels, the photodiodes in CMOS sensors can be controlled by MOS-FET (Metal Oxide Semiconductor Field Effect Transistor). Their specific architecture allows the combination of the electro-optical process with further electronic processing of the signal directly on-chip, (RAFFEL et al., 2018).

The principle of a CMOS sensor is shown in Figure 4.9. Each pixel contains an electronic circuit. 
Figure 4.9 - Model of a CMOS sensor.

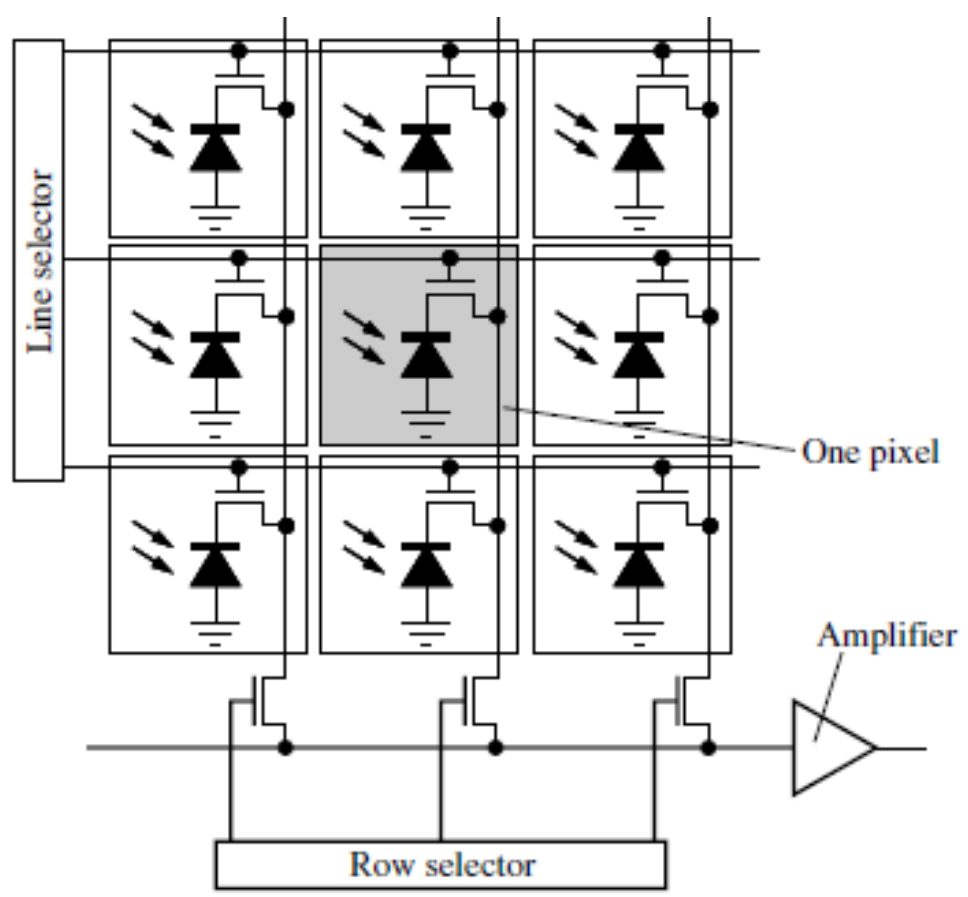

Source: Raffel et al. (2018).

CMOS sensors for PIV applications are based on the active pixel sensors (APS) technology in which, in addition to the photodiode, a readout amplifier is incorporated into each pixel. This amplification converts the charge accumulated by the photodiode into a voltage, which is amplified inside the pixel and then transferred in sequential rows and columns to further signal processing circuits. Besides, some CMOS sensors contain shutter transistors for each pixel; this kind of transistors control the activation for each pixel to capture the light at the right time set in the camera parameters. The amplifier transistor represents the input device of what is generally termed as a source follower. It converts the charge generated by the photodiode into a voltage that is the output to the column bus. The reset transistor controls integration time, and the row-select transistor connects the pixel output to the column bus for readout.

According to Raffel et al. (2018), the pixel architecture, together with the individual access to each pixel, offers some significant advantages and allow to integrate fundamental camera function like amplification, nonlinear signal transformations, and AD-conversion on-chip. The CMOS sensors can be configured in long electrical lines for each row and column. That means larger sensors which are usually needed for PIV applications.

\subsubsection{Method for Image Acquisition}

For image recording, there are two categories: (1) methods that capture the illuminated flow on to a single frame and (2) methods that provide a single illuminated image for each illumination pulse, (RAFFEL et al., 2018). These branches are referred to as single frame/multi-exposure PIV and multi frame/single exposure PIV. The single-frame/multi-exposure PIV recording was first utilized in conjunction with photography. Multiple frame/single exposure has been developed after years, and nowadays, it is possible 
to use this technique with a PIV system using high-speed motion cameras. Also, some systems acquire images using multiple frames/double exposure.

When using photographic film or single frame digital cameras for PIV recording, two or more exposures from the single frame are stored on a single recording. Instead, the multi-frame PIV recording stores each exposure into individual frames. Separating the light of the subsequent recordings in different frames allows the adaptation of the pulse separation time into a broader range, also higher signal-tonoise ratios in the correlation plane allows to compute the displacement with smaller interrogation areas, therefore increases the spatial resolution of the recording (RAFFEL et al., 2018; STANISLAS et al., 2003). An schematic comparison between the different recording methods are shown in Fig. 4.10.

Figure 4.10 - Single frame techniques are shown at upper side, for: (a) single exposure. (b) double exposure. (c) multiple exposure. At the bottom side are shown the two most common multiple frame techniques: (d) double frame with single exposure and (e) double frame with double exposure. The open circles indicate the particle position in the previous frame.

(a)

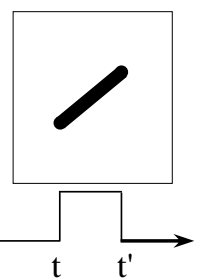

(b)

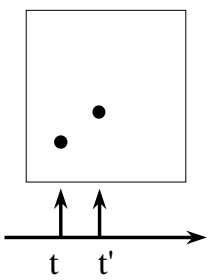

(c)

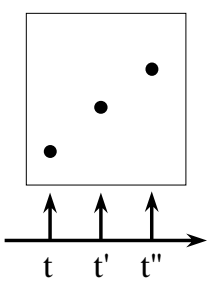

(d)

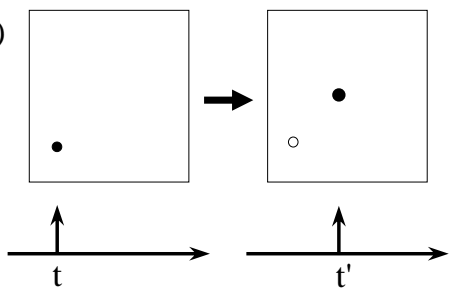

(e)

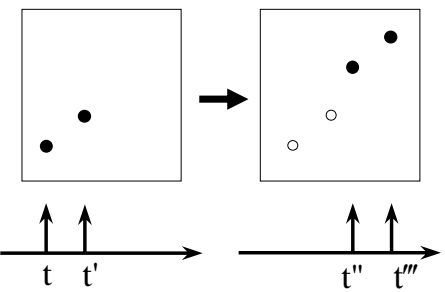

Source: Adapted from Raffel et al. (2018).

In the single frame/multi-exposure recording, it is not easy to determine the sign of the particle direction during the motion within each interrogation area since there is not a way to decide which image is the first or the second illumination pulse. In some applications, it is necessary to have a priori knowledge of the flow, and multi-frame solves this directional ambiguity, and as it was mentioned allows to record high-speed phenomena.

In this project, Dynamic Studio software allows setting a single or multi-frame mode for the PIV system. Due to the characteristics of the present study, it is used a simple multiple frame/single exposure technique to acquire images, this is a double frame with a single exposure mode, that allows acquiring a set of pair images. This configuration guarantees a good correlation and minimizes the noise error. Figure 4.11 shown a schema for this image sampling technique.

\subsubsection{Image Correlation}

Images are considered as signals, and the first image may be considered the input to a system whose output produces the second image of the pair, as it is shown at Figure 4.12, it is the model of a digital signal processing for two successively recorded particle image frames. Here, the transfer function of the system, 
Figure 4.11 - Conceptual description of a double frame single exposure Particle Image Velocimetry.

Image 1

Time $=t$

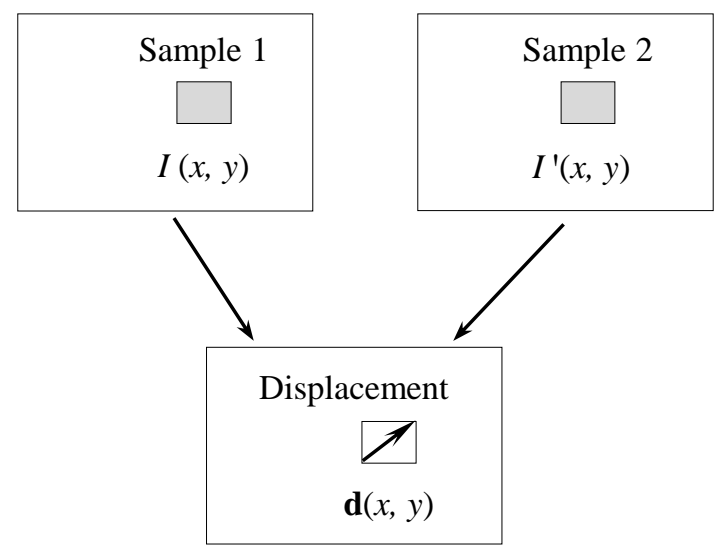

Field of estimated displacement

Image 2

Time $=t+\Delta t$

Source: Adapted from Raffel et al. (2018).

$\mathbf{H}$, converts the input image $I$ to the output image $I^{\prime}$ and is comprised of the displacement function $\mathbf{d}$ and additive noise process, $\mathbf{N}$. The function $\mathbf{N}$ models the effect due to the noise recorded and the addictive process for three-dimensional flows. If the functions $\mathbf{d}$ and $\mathbf{N}$ are known, it is possible to use them as transfer function for the input image $I$ to produce the output image $I^{\prime}$. With both images known, the aim is to estimate the displacement field $\mathbf{d}$ while excluding the noise. The images are not continuous, and it is necessary to estimate the displacement using a statistical approach based on interrogation windows or samples, (RAFFEL et al., 2018).

Figure 4.12 - Linear digital signal processing model describing the functional relationship between two successively particle image frames.

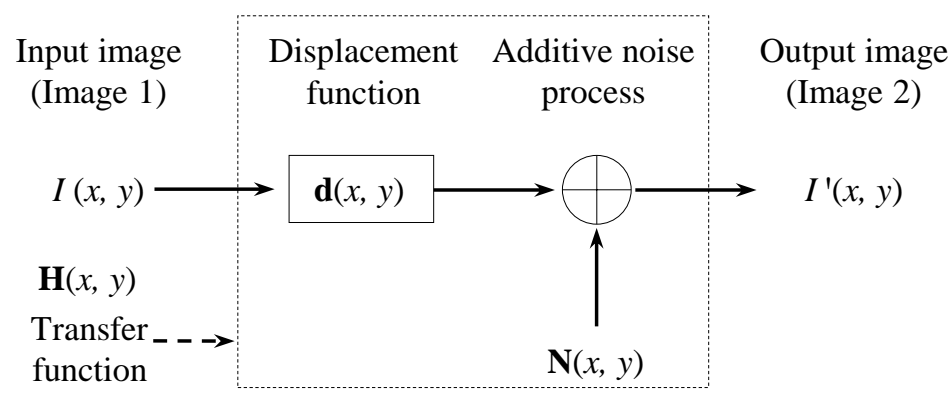

Source: Adapted from Raffel et al. (2018).

One possibility to know the local displacement is to deconvolve the pair of images. This is accomplished by dividing the Fourier transforms by each other. The method is useful when the noise in the image is insignificant. However, the noise associated with realistic recording conditions quickly degrades the data accuracy.

Rather than estimating the displacement function $\mathbf{d}$ analytically, the method is to correlate the images. 
It is to find the best match between the images in a statistical sense; this is accomplished using the discrete cross-correlation function (RAFFEL et al., 2018), given by:

$$
R_{I I}(x, y)=\sum_{i=-K}^{K} \sum_{i=-L}^{L} I(i, j) I^{\prime}(i+x, j+y)
$$

the variables $I$ and $I^{\prime}$ are the samples extracted from the images where $I^{\prime}$ is larger than template $I$. Essentially the template $I$ is linearly shifted around in the sample $I^{\prime}$. For each choice of sample shift $(x, y)$, the sum of the products of all overlapping pixel intensities produce one cross-correlation value $R_{I I}(x, y)$. By applying this operation for a range of shifts $(-M \leq x \leq+M,-N \leq y \leq+N)$, a correlation plane is the size of $(2 M+1) \times(2 N+1)$ is formed. For values of shifting at which the samples' particle images align with each other, the sum of the products of pixel intensities is larger than elsewhere, resulting in a high cross-correlation value $R_{I I}$ at this position (RAFFEL et al., 2018).

Essentially the cross-correlation function statistically measures the degree of match between two samples for a given shift. The highest value in the correlation plane can then be used as a direct estimate of the particle image displacement.

The alternative to calculating the cross-correlation directly using equation (4.8) is using the correlation theorem which states that the cross-correlation of two functions is equivalent to a complex conjugate multiplication of their Fourier transforms:

$$
R_{I I} \Leftrightarrow \hat{I} \cdot \hat{I}^{\prime}
$$

where $\hat{I}$ and $\hat{I}^{\prime}$ are the Fourier transforms of the functions $I$ and $I^{\prime}$, respectively.

Since the input data for calculating the correlation function are discrete and integer values such as the intensity of the images and their position, the value of the correlation peak is also an integer. Thus, this function allows calculating the position of the correlation point and its displacement with an uncertainty of $\pm 1 / 2$ pixels.

\subsubsection{Peak Detection and Displacement Estimation}

A crucial feature of the PIV evaluation is the correlation peak for sub-pixel accuracy. For interrogation areas of $32 \times 32$, the estimated accuracy is the order of $1 / 10^{\text {th }}$ to $1 / 20^{\text {th }}$ for 8 -bit digital images. In order to determinate the correlation peaks and the displacement, in this project is used the features of the Dynamic Studio software (DYNAMICS, 2015). The software detects the highest correlation peaks. Each peak is set with coordinates $x_{m}$ and $y_{m}$ at the correlation plane, and a two-dimensional function is used in order to calculate the exact position of the peak. Assuming a two-dimensional Gaussian curve, the interpolation function is given by:

$$
f(x, y)=h \times \exp \left(-4 \frac{\left(x-x_{0}\right)^{2}+\left(y-y_{0}\right)^{2}}{w^{2}}\right)
$$

where $\left(x_{0}, y_{0}\right)$ is the maximum correlation point, $h$ and $w$ are the height and the width of the peak correlation, respectively. Normally $w$ can be determined with an expression derived from the standard deviation $\sigma$, expressed as:

$$
w=2 \sqrt{2 \sigma}
$$


As it is represented in the figure 4.13, for calculation of the peak correlation coordinates the highest correlation point is evaluated and its neighboring points as well.

Figure 4.13 - Two-dimensional map for calculating the position of the peak correlation. Rows represent the $\mathrm{x}$-coordinate and columns the $\mathrm{y}$-coordinate for the peak correlation point, and Max is the point at the maximum correlation value.

\begin{tabular}{|c|l|l|l|l|}
\hline & $\mathrm{x}_{\mathrm{m}}-1$ & $\mathrm{X}_{\mathrm{m}}$ & $\mathrm{x}_{\mathrm{m}}+1$ & \\
\hline $\mathrm{y}_{\mathrm{m}}+1$ & & & & \\
\hline $\mathrm{y}_{\mathrm{m}}$ & & Max & & \\
\hline $\mathrm{y}_{\mathrm{m}}-1$ & & & & \\
\hline
\end{tabular}

Source: Raffel et al. (2018).

For the purpose of evaluating the highest correlation point, the expression (4.10) is modified replacing $\exp =(1+x)$, then it is obtained:

$$
f(x, y)=h\left(1-4 \frac{\left(x-x_{0}\right)^{2}}{w_{x}^{2}}\right)\left(1-4 \frac{\left(y-y_{0}\right)^{2}}{w_{y}^{2}}\right)
$$

where the expressions $w_{x}$ and $w_{y}$ are the standard deviation for each direction $(x$ and $y)$. In order to set the peak correlation point, an analysis for bi-dimensional function is required using equation (4.12). At direction $x$ the values $\left(x_{0}, y_{m}\right)$ are used in Eq.(4.12) obtaining a correlation value $\left(h_{y_{m}}\right)$, given by:

$$
f(x, y)=h\left(1-4 \frac{\left(y_{m}-y_{0}\right)^{2}}{w_{y}^{2}}\right)
$$

In the same way, the correlation values for the neighboring points at direction $x$ are calculated with the following equations:

$$
\begin{gathered}
f\left(x_{m}-1, y_{m}\right)=h_{y_{m}}\left(1-4 \frac{\left(\left(x_{m}-1\right)-x_{0}\right)^{2}}{w_{x}^{2}}\right) \\
f\left(x_{m}, y_{m}\right)=h_{y_{m}}\left(1-4 \frac{\left(x_{m}-x_{0}\right)^{2}}{w_{x}^{2}}\right) \\
f\left(x_{m}+1, y_{m}\right)=h_{y_{m}}\left(1-4 \frac{\left(\left(x_{m}+1\right)-x_{0}\right)^{2}}{w_{x}^{2}}\right)
\end{gathered}
$$

After calculating these values, a parabolic interpolation of the Gaussian curve can be found in the following values:

$$
x_{0}=x_{m}+\frac{f\left(x_{m}+1, y_{m}\right)-f\left(x_{m}-1, y_{m}\right)}{4 f\left(x_{m}, y_{m}\right)-2 f\left(x_{m}-1, y_{m}\right)-2 f\left(x_{m}+1, y_{m}\right)}
$$




$$
\begin{gathered}
h_{y m}=f\left(x_{m}, y_{m}\right)+\frac{1}{4}\left(f\left(x_{m}+1, y_{m}\right)-f\left(x_{m}-1, y_{m}\right)\right)\left(x_{0}-x_{m}\right) \\
w_{x}^{2}=\frac{8 h_{y_{m}}}{2 f\left(x_{m}, y_{m}\right)-f\left(x_{m}-1, y_{m}\right)-f\left(x_{m}+1, y_{m}\right)}
\end{gathered}
$$

The same process is repeated for calculations at $y$ direction. The correlation peak $h$ is calculated with corrections by the interpolation at directions $x$ and $y$, at the central point value $f\left(x_{m}, y_{m}\right)$. The value of $w$ is obtained by: $w=\left(w_{x}^{2} \times w_{y}^{2}\right)^{0.25}$.

Figure 4.14 - Correlation peak function (c) calculated for an interrogation area of $32 \times 32$ using a double frame (a) and (b) - single exposure method. Correlation made at $R e=24600$ for water flow.
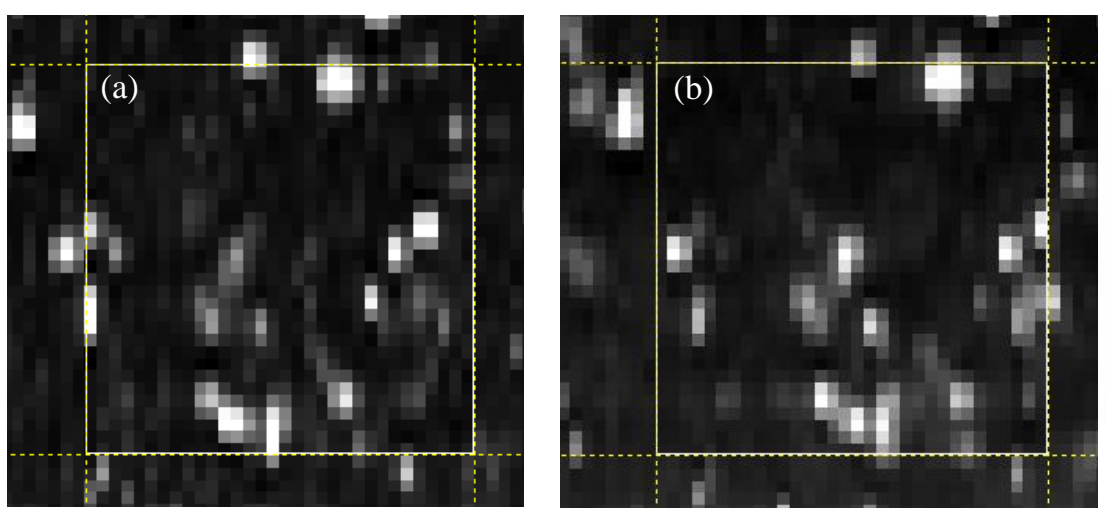

(c)

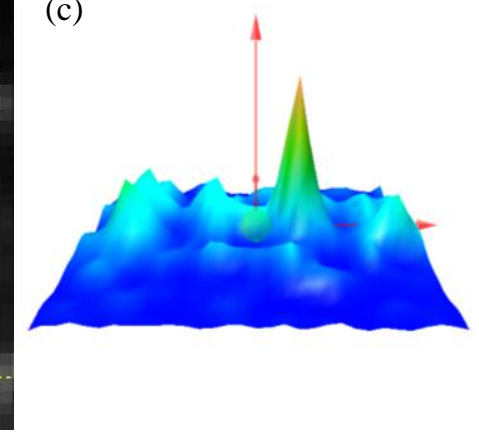

Source: Author (2019).

Finally, the position for the correlation peak is calculated with the accuracy of the order of the sub-pixels. This position is defined as the mean displacement of the particles in the interrogation area analyzed, and the smaller values of correlation are assumed as the noise presents in the image acquired. Figure 4.14 shows a real evaluation for the correlation peak for a water flow at $R e_{D}=24600$, where the correlation function $\left(R_{I I}\right)$ for a pair of images of a $32 \times 32$ interrogation area is evaluated. The highest peak correlation value is found, and it is represented into a Gaussian distribution.

\subsubsection{Adaptive Interrogation Technique}

Adaptive interrogation is an iterative multi-grid technique that increases the spatial resolution by decreasing the final window size. In several cases, the flow and the distribution of seeding particles are not homogeneous over the observed domain. In this situation, there are not optimal conditions due to a weak correlation signal, and too low flow sampling rate may occur. The window filtering effect can be minimized when the flow has variations along a direction. An adaptive correlation also contributes to achieve further improvements when dealing with vortex structures.

It is possible to apply the adaptive interrogation technique with different features within Dantec Dynamics software. The features for adaptive correlation can use the criterion of overlapping for the 
interrogation areas (IA), the offset method or windows displacement, and the multiple pass interrogation method. Every method is explained in detail at the next subsections.

\subsubsection{Overlap method}

The adaptive correlation allows the overlapping of the interrogation area as a function of its size. Normally, the overlapping values used for adaptive correlation are 25, 50, and $75 \%$. The overlapping criterion increases the number of vector for each interrogation area. Figure 4.15 presents an interrogation area with an overlap of $50 \%$, calculating 5 vectors for the IA. Otherwise, if a simple correlation is made, with an overlap of $0 \%$, it would be calculated just one vector for the area under analysis. It is clearly shown that the overlap method represents an advantage in obtaining better correlations between the IA's of the pair of images.

Figure 4.15 - Overlap method applied for a interrogation area. The area was overlapped $50 \%$ at the neighboring areas. Retrieved from Dynamics (2015).

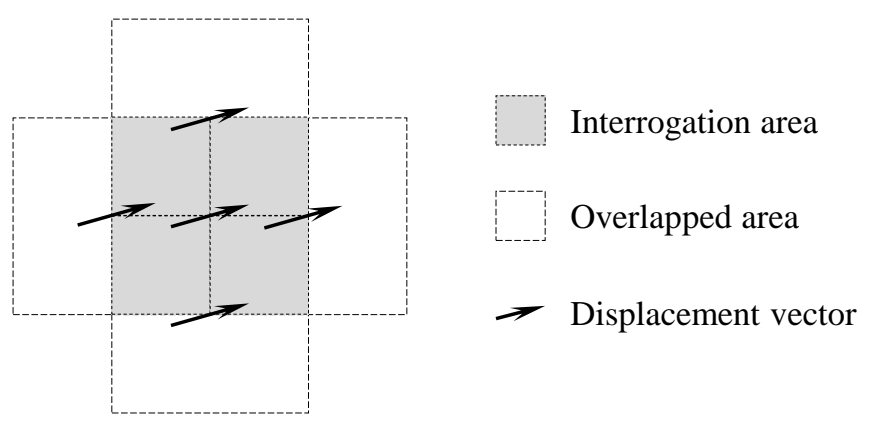

Source: Adapted from Raffel et al. (2018).

The parameter overlap in the Dantec Dynamics software also allows setting independently for the horizontal and vertical direction. It offers total freedom to increase vector map resolution in any direction, reducing spurious vectors. This kind of vector can be identified as having an unusual behavior when it is compared with its neighboring vectors. These spurious vectors are outliers with random values that do not follow the same pattern in a vector field matrix.

\subsubsection{Multiple pass interrogation and offset method}

Multiple pass interrogation method allows getting the intermediate information from an initial interrogation area size, as information of a next smaller window. The process is repeated until it reaches the final interrogation area size. In practical applications, it is normal to uses a refinement of the interrogation area of 2 or 3 times until the final area size is reached. For an interrogation area of $32 \times 32$ pixels, initial interrogation areas of $256 \times 256$ pixels are used (RAFFEL et al., 2018; PALACIOS, 2011).

The data yield in the interrogation process can be significantly increased by using a window offset equal to the local integer displacement in a second interrogation pass. By offsetting the interrogation area according to the mean displacement, the fraction of matched particle images is increased, which 
means that the signal/noise ratio of the correlation peak is increased. Also, the measurement noise and uncertainty in the displacement reduces significantly when the particle image is less than a pixel $(\mathbf{d}<0.5$ pixel) (RAFFEL et al., 2018).

The offset method has been used in the last digital PIV generation to maximize spatial resolution and to eliminate systematic errors. The present study is developed using an interrogation area offset produced by a central difference interrogation scheme (CDI) to calculate the vectors. This scheme has an error of the order of $(\Delta t)^{2}$; this allows greater accuracy when compared to forward difference interrogation scheme (FDI), which has an error of $\Delta t$ (RAFFEL et al., 2018). Figure 4.16 presents both interrogation schema.

Figure 4.16 - Sampling interrogation area using: (a) central difference interrogation (CDI) scheme. (b) forward difference interrogation (FDI) scheme.
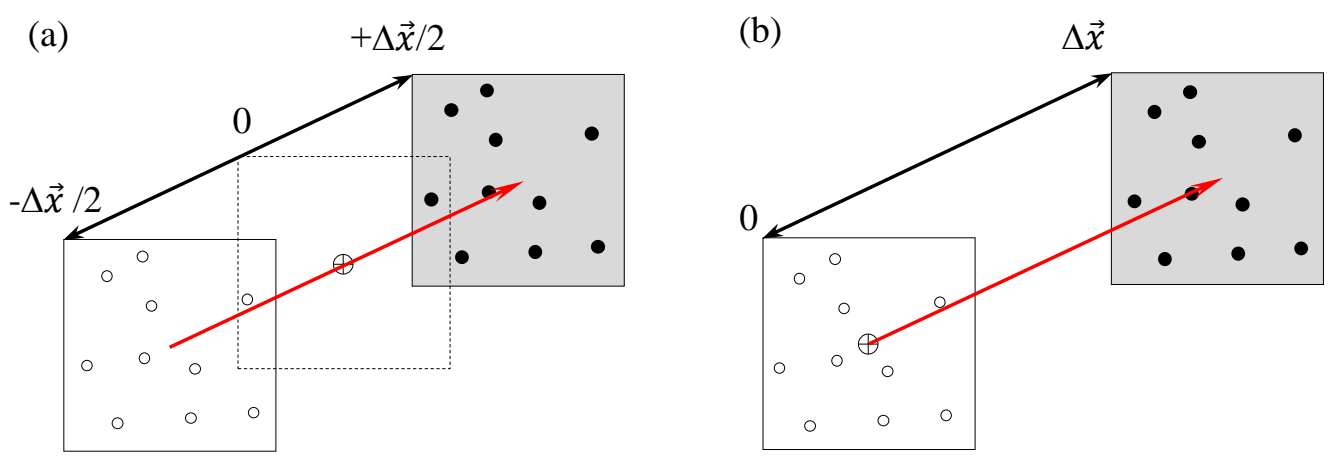

Source: Adapted from Raffel et al. (2018).

The CDI scheme has more advantages than the FDI scheme; this method is mathematically the most accurate methodology and, therefore, it shall be preferred with PIV measurements as the measurements required for the present study. On the other hand, Dynamics (2015) recommends, when processing, further advanced measurements such as PIV/LIF, the FDI Scheme shall be used because the LIF-image gets the same temporal reference $\left(t_{0}\right)$ as the velocity vector map, which is not the case with the CDI scheme. According to Wereley and Meinhart (2001), the CDI allows measuring closer to flow boundaries and is more symmetric than the FDI scheme. So, CDI is the most suitable interrogation scheme to obtain a better correlation near to the pipe wall and near the corner of the contraction.

\subsection{UNCERTAINTY ANALYSIS}

The accuracy of the system depends on many factors as the equipment sophistication to perform the measurements, physical principles of the measuring instrument, the skill of the experimentalist, physical properties of the phenomenon, and knowledge about the values to determine at the measuring process.

In this experimental study, uncertainties arise owing to the equipment involved and the propagation of error in the quantities that can be derived through the calculations process. The variables measured in this study are pressure, volumetric flow rate, viscosity, temperature, density, the length between pressure ports, and pipe diameters. 


\subsubsection{Uncertainty Analysis for Measurement Devices}

In order to determine the uncertainty value for the instruments, the recommendations from the manufacturer will be considered. So, depending on the instrument and its operating range, the uncertainty value is obtained. For quantities obtained through calculation operations, such as $R e, V, Q$, among others, there will be considered the magnitude and error or each variable involved. Coleman and Steele (2018) indicate that for a magnitude defined by:

$$
G=f\left\{x_{1}, x_{1}, x_{1}, \ldots, x_{M}\right\}
$$

where $M$ is the number of independent variables, so the true value for the magnitude $G$ is given by:

$$
G=\bar{G} \pm \delta(G)
$$

where $\bar{G}$ is the average value defined as $\bar{G}=\left\{\bar{x}_{1}, \bar{x}_{2}, \bar{x}_{3}, \ldots, \bar{x}_{M}\right\}$, and $\delta(G)$ is the uncertainty of $G$. This uncertainty value is defined by Coleman and Steele (2018) by the following equation:

$$
\delta(G)= \pm\left\{\sum_{i=1}^{M}\left[\left.\left(\frac{\partial G}{\partial x_{i}}\right)\right|_{x_{i}=\bar{x}} \delta\left(x_{i}\right)\right]^{2}\right\}^{1 / 2}
$$

where the expression $\delta\left(x_{i}\right)$ is the uncertainty value for the variable $x_{i}$, and $\partial G / \partial x_{i}$ is the coefficient of sensitivity, which describes how the value of $G$ varies with changes of the variable $x_{i}$. The uncertainty value and coefficient of sensitivity can be obtained from the specification data given by the manufacturer of each instrument involved in the measurement process. The analysis of uncertainty for the different variables measured in this study is presented in B.1, including the uncertainty for pressure measurements in function of the Reynolds number and for each viscoplastic fluid.

\subsubsection{Uncertainty Analysis for PIV Technique}

One of the sources of experimental errors in the use of the PIV technique arises from the calculation of the flow parameters such as the velocity profiles. The main errors in the 2D PIV technique are related to image correlation, pixel locking error, and systematic or Bias error. Other parameters as the number of vectors and the frequency of acquisition, which refers to the spatial and temporal resolution, are very significant to calculate the uncertainty. In the literature, it is possible to find some criteria that express how the parameters affect the accuracy of the 2D PIV technique (WESTERWEEL et al., 1996; WESTERWEEL, 2000; RAFFEL et al., 2018).

Westerweel (2000) determined that the uncertainty for the PIV technique is minimized when there is enough number of particles $\left(N_{p}\right)$ in the interrogation areas, and the fraction of particles in two interrogation areas $\left(F_{p}\right)$. Doorne and Westerweel (2007) recommend the use of $N_{p} \geq 10$ and $F_{p} \geq 0.75$, to reach an uncertainty value of 0.1 pixel for the particle displacement.

A type of error that occurs when the average image diameter of the particle $\left(d_{\tau}\right)$ is less than 2 pixel is the pixel locking or peak locking error. This error occurs because the software it is no able to correlate the particle displacement, and when the particle-image diameter falls between one and two-pixel units, the 
peak locking is usually within acceptable limits, which can be referred to a mild peak locking. Severe pixel locking occurs when the particle image diameter falls below one-pixel unit, and normally this error has a magnitude near to 0.01 pixel. (WESTERWEEL, 2000; CHRISTENSEN, 2004; OVERMARS et al., 2010). In order to avoid the peak locking error Doorne and Westerweel (2007) suggested a $d_{\tau}$ between 2 and 4 pixel.

Since the images acquired by the camera have slight distortions, due to the curvature of the tube and the difference between the material refractive indexes, this effect must be corrected. For this purpose, a dewarping process is available in Dynamic Studio software, which is a third-order polynomial function that is used as a mapping resource and allows to find distortions on the image plane from calibration images. This function detects barrel effects caused by the distortions and adjusts the images according to the visualization plane. The dewarping function also includes the errors of the particle position, which is typically close to 1 pixel. Besides, there are some errors in the particles near to the wall; for that reason, some vectors near to the wall are calculated with slightly wrong positions. This lead to a position error of 6 pix $/ 7000$ pix $\approx 0.35 \%$. Statistical errors due to the number of vectors are not merely determined by the quantity of the set of data or samples (WESTERWEEL, 2000; DOORNE; WESTERWEEL, 2007).

An appropriate way to link the size the data set to the sampling uncertainty is to consider the equivalent measurement time expressed in integral time scales. The measurement time is estimated by dividing the total length of the data set in the axial direction (size of the view area in the axial direction multiplied by the number of recorded images) by the average velocity at the test section entrance $(\bar{U})$. The integral time scale may be estimated by dividing the pipe diameter by this average velocity $(D / \bar{U})$. So, the sampling error for the flow statistic is inversely proportional to the square root of the corresponding measurement time (WESTERWEEL et al., 1996). The following equations show the different expressions for these parameters,

- Measurement time:

$$
M_{t}=\frac{X_{D} N_{T}}{\bar{U}}
$$

where $N_{T}$ is the total of images captured, $X_{D}$ is the length of the view size in the axial direction.

- Integral time scale:

$$
I_{t}=\frac{D}{\bar{U}}
$$

- Sampling error:

$$
e_{s}=\frac{1}{\sqrt{\frac{M_{t}}{I_{t}}}}
$$

Equation (B.13) demonstrates the importance of collecting a large number of pair of images. In this project the number of pair of images is limited by the PIV system, this is set at the maximum capacity of 1040 pair of images, that means 2080 images for each test. The uncertainty values for the image acquisition are presented in Appendix B.2 for the different Reynolds numbers and fluids.

Other way to evaluate the uncertainty of the data obtained by the PIV technique is calculating the 
mean error for each sample by the expression:

$$
\sigma_{\bar{X}}= \pm \frac{2 \sigma_{X}}{\sqrt{N_{X}}}
$$

where $\sigma_{X}$ is the standard deviation for a measured variable of $X$, and $N_{X}$ is the number of samples for $X$, (CARR et al., 2009).

This bias error is evaluated considering the standard deviation of the different variables analyzed, according to the criteria given by Carr et al. (2009) for PIV spatial correlation. This definition of uncertainty is the basis for the calculation of turbulent fluctuations or $r m s$ velocities presented in section 5.2.4

\subsection{MEASURING PROCESS}

This section is focused on the two types of measurements performed in the present study: the pressure measurements along the flow-loop to calculate the pressure loss coefficient. And the velocity vectors and its derived quantities obtained through the PIV technique, such as radial and axial velocities and turbulent fluctuations.

\subsubsection{Pressure Measurements and Pressure Loss Coefficient Calculation}

Along the upstream and downstream regions of the contractions, the static pressure was measured for different Reynolds number set for each viscoplastic fluid. The pressures values obtained were used to determine the pressure loss coefficient. Figure 4.17 shows in detail the setting of pressure transmitter, previously shown in Figure 4.3.

Figure 4.17 - Measuring of pressure along the flow-loop

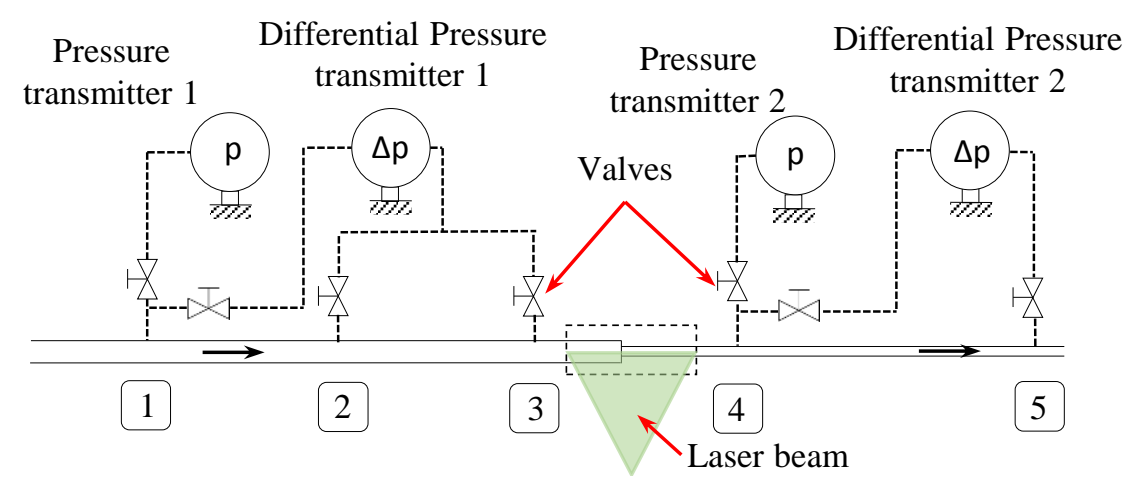

Source: Author (2019).

For measuring the pressure at position 2 and 3, it is used a differential manometer. To obtain the pressure at point 2 , the valve at this point was opened while at point 3 , it was closed. This assured to measure just the differential pressure at points 1 and 2 . Thus, the pressure at point 1 was known, the static pressure at point 2 was obtained using the differential pressure value between point 1 and 2 . The same 
methodology was applied to obtain the pressure at point 3. It was repeated to get the pressure data from the upstream region, at points 4 and 5, considering the static pressure from point 4 measured by a pressure transmitter 2 .

The table shows the distance between the points where the pressure was measured, where $L_{i j}$ represents the length between the points $i$ and $j$. The contraction plane is a reference location to extrapolated the pressure from points 3 and 4 , using the variables $L_{3-\text { con }}$ and $L_{c o n-4}$ which are the length between these points and the contraction plane.

Table 4.8 - Distance between the pressure measuring points

\begin{tabular}{l|l}
\hline $\mathbf{i j}$ & $\mathbf{L}_{i j}[\mathbf{m}]$ \\
\hline $1-2$ & 0.97 \\
$2-3$ & 1.00 \\
$3-\operatorname{con}$ & 0.37 \\
con -4 & 0.70 \\
$4-5$ & 0.90 \\
\hline \multicolumn{2}{l}{ Source: Author (2019). }
\end{tabular}

Source: Author (2019).

After recorded all the pressures, it was applied linear regression to extrapolate the values until were obtained an upstream and downstream pressure at the contraction. These pressures were set as $P_{c 1}$ and $P_{c 2}$, and then, the loss pressure at the contraction was calculated by:

$$
\Delta P_{\text {con }}=P_{c 1}-P_{c 2}
$$

The value obtained for $\Delta P_{\text {con }}$ was replaced at the equation (2.51) to obtain the value for the loss coefficient at the contraction $\left(k_{\text {con }}\right)$.

\subsubsection{Vectors, Velocity Fields and Derived Quantities}

As previously explained, to obtain the velocity fields, the images obtained were post-processed using the Dynamic Studio software. The distortions, due to the curvature of the pipe and the difference between the refractive indexes of the pipe and the type of fluid were corrected with a calibration process made through a Dewarping function. This calibration process also allows setting masks, and limits on the images acquired and to define the area to be evaluated.

After the calibration process, it was delimited the area visualized with dimensions of $52 \mathrm{~mm} \times 26$ $\mathrm{mm}$, divided by interrogation areas of 52 pixels $\times 26$ pixels. This relation leads to obtain images with approximately 36 pixels $/ \mathrm{mm}$ or the equivalent measure of 1 pixel $=0.027 \mathrm{~mm}$. Due to the overlap method applied in this study, the density of vector in the area visualized is increased. Then, there is a vector point, each $0.432 \mathrm{~mm}$ of the area visualized. This measure can be scaled by $D$, obtaining a distance between vectors equivalent to $0.0169 D$.

The image acquisition was performed using the experimental setup shown in section 4.3. The laser beam light reached the meridional plane of the contraction, located inside the visualization box, as it was 
shown in figure 4.5. The correlations were obtained capturing the seeding particles at two instants of times.

Figure 4.18 - Process for image acquisition and post-processing steps with Dynamic Studio software.

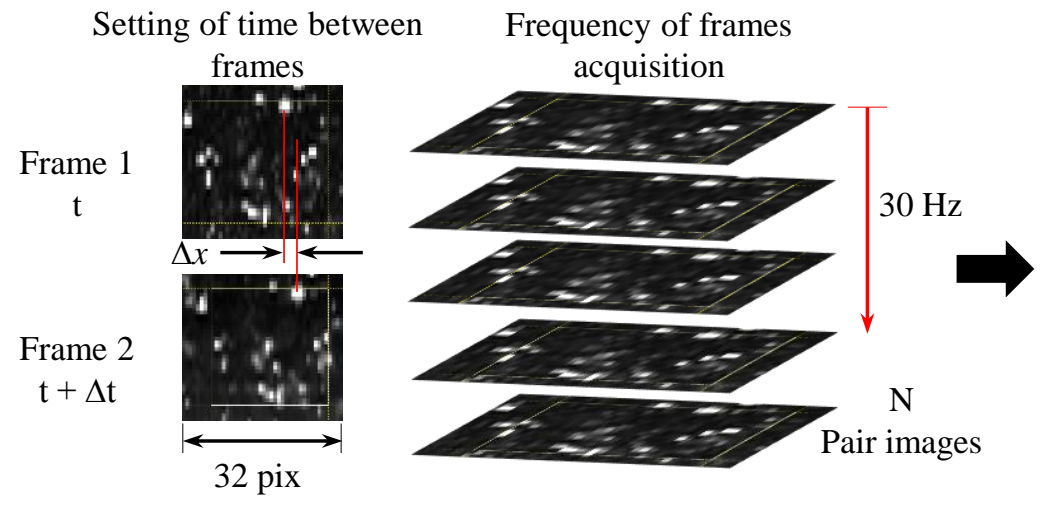

Correlation process
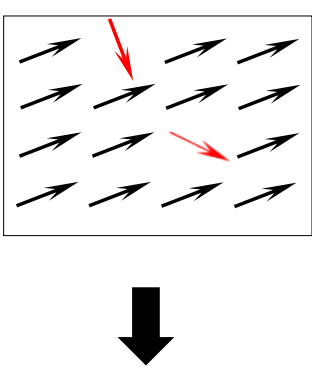

Spurious vector substitution

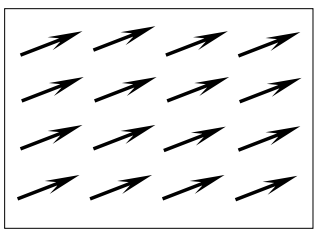

Images to be correlated

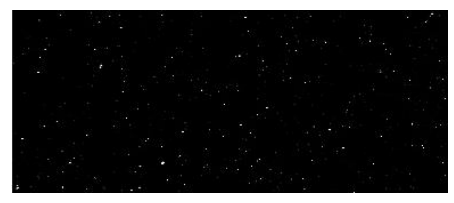

Vector statistic process

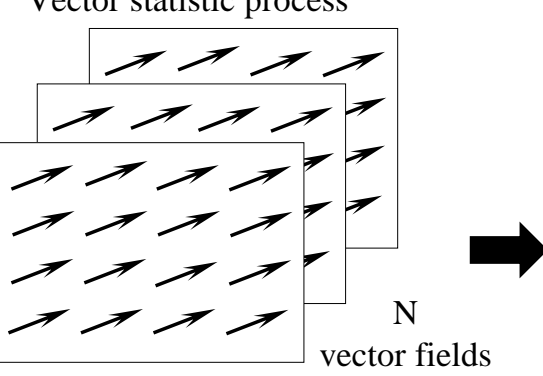

Dewarping and normalization process
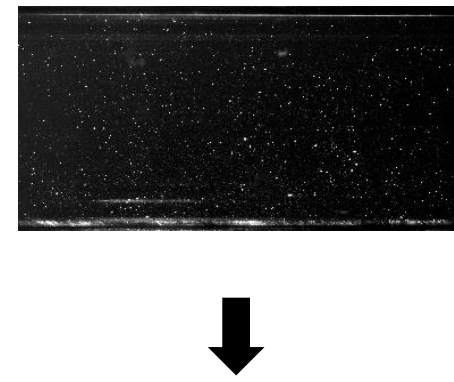

Masking process

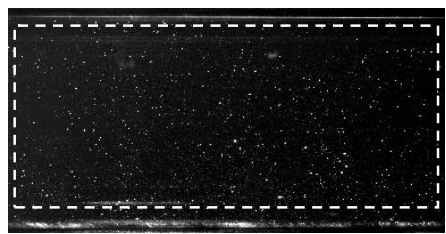

Mean flow and turbulence quantities

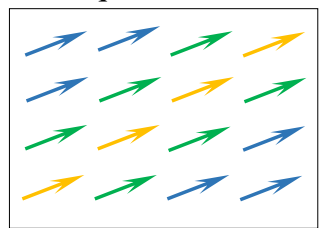

Source: Author (2019).

The synchronization between the laser and the camera was set by the Dynamic Studio software, assuring the laser beam is produced at the same time that the camera shutter is opened. Also, the acquisition frequency and the time between pulses was set according to the required mean velocity at the entrance of the test section. Initial tests with Newtonian fluid were performed for evaluating the PIV methodology. For these tests some parameters were set according to the Reynolds numbers, and the mean flow quantity: velocity field, and the first orders turbulent quantities were obtained for turbulent Reynolds numbers. The data are compiled and analyzed in Appendix A.

For turbulent first-order quantities, the frequency of acquisition data was chosen as being higher or equal to the integral time scale, defined at section 4.6 as the ratio between the diameter of the pipe and the average flow velocity. For the initial validation with Newtonian fluid into a straight pipe, the frequency was set at 50, and $100 \mathrm{~Hz}$; Doorne and Westerweel (2007) and Ozalp et al. (2007) recommended the 
use of a frequency equal or higher than $15 \mathrm{~Hz}$ For the time between pulses, the average displacement of the seeding particles into an interrogation area was calculated to set a suitable time between pulses that allows a good correlation, also it is used for definition of the integral time scale $I_{t}$ described in Appendix B.2. The $I_{t}$ value is set as the upper limit of the time between pulses. The $R e$ were set based on the Malin (1998) criterion $\left(R e_{D}^{\prime}\right)$, which is the best correlation of $R e$ for Herschel-Bulkley fluids. Table 4.9 summarized all the parameters set for visualizing the different solutions at different $R e_{D}^{\prime}$.

Table 4.9 - Variables set to visualize fluid flow with the PIV technique

\begin{tabular}{llccc}
\hline Fluid & $R e_{D}^{\prime}$ & Time between pulse $[\mu \mathbf{s}]$ & Frequency $[\mathbf{H z}]$ & Exposure time $[\mu \mathbf{s}]$ \\
\hline 10W & 1500 & 600 & 50 & 1000 \\
& 2000 & 450 & 50 & \\
& 4000 & 230 & 50 & \\
& 5000 & 190 & 100 & \\
& 10000 & 95 & 100 & 2500 \\
\hline 20W & 10 & 2000 & 50 & \\
& 50 & 600 & 50 & \\
& 100 & 350 & 50 & \\
& 200 & 210 & 50 & \\
& 500 & 250 & 100 & \\
& 1000 & 130 & 100 & \\
& 1500 & 100 & 100 & \\
\hline $30 \mathrm{~W}$ & 10 & 900 & 50 & \\
& 50 & 300 & 50 & \\
& 100 & 190 & 50 & \\
& 200 & 120 & 50 & \\
& 300 & 90 & 50 & \\
& 500 & 65 & 100 & \\
& 650 & 50 & 100 & \\
\hline $40 \mathrm{~W}$ & 1 & 2500 & 50 & \\
& 10 & 600 & 50 & \\
& 100 & 128 & 50 & \\
\hline
\end{tabular}

Source: Author (2019).

From the image acquisition process scalar maps of the fluid flow behavior of the mean flow and first turbulent quantities were obtained, also the correlation of these with changes in the shear stress, shear rate and viscosity along entrance region of the contraction. A comparison of the results is made for each solution at the different flow regimes. The mean statistic flow and turbulent quantities can be listed as follow:

\section{Mean flow quantities:}

- Axial component (u)

- Radial component $(v)$ 
- Centerline velocity $\left(u_{C L}\right)$

\section{Turbulent quantities:}

- Turbulent fluctuations $\left(u^{\prime}, v^{\prime}\right)$

\section{Changes at the unyielded regions}

\section{Vortex structures dimensions}

Simultaneously to the image acquisition process, values of flow parameters such as volumetric flow, density, temperature at inlet and outlet section were acquired through the LabVIEW command, which captures the data provided by the equipment used. Once the time between pulses and the frequency were set, the camera acquired the images. In addition to the Dewarping feature, depending on the resolution of the acquired images, it is appropriate to carry out a further normalization process. During the acquisition of the images, sections of the tube wall are usually captured to delimit and facilitate the subsequence treatment. The images were cut out, defining the section of interest with a masking feature. Afterward, the velocity vectors were exported as numerical values for plotting process and analysis. Figure 4.18 shows in detail the sequence for the images acquisition and post-processing stages.

\subsection{FRICTION FACTOR VALIDATION}

In order to validate the different regimes for the viscoplastic flow, the friction factor was calculated for each Reynolds numbers set for the different viscoplastic solution, and based on (MALIN, 1998) criterion. The friction factor is based on Fanning correlations, and it is related to the pressure drops along a straight tube section. For this validation the pressure drop values between points 2 and 3 of the flow loop were obtained. The distance from these points from the contraction is 1.37 and $0.37 \mathrm{~m}$, respectively. At these distances, there are not contractions effects on the flow.

According to Metzner and Reed (1955) correlations for the experimental friction factors can be calculated using the following mathematical expression:

$$
f=\frac{2 \tau_{w}}{\rho \bar{U}^{2}}
$$

where $\tau_{w}$ is defined by equation (2.33).

The experimental data are compared with the correlation given by Malin (1998), for the laminar and turbulent regimes. These expressions were defined at section 2.2, retrieved here for convenience. For laminar flows $f$ is expressed by:

$$
f=\frac{16}{\Psi R e^{\prime}}
$$

where $\Psi$ is defined by:

$$
\Psi=(1+3 n)^{n}(1+\phi)^{(1+n)}\left[\frac{(1-\phi)^{2}}{(1+3 n)}+\frac{2 \phi(1-\phi)}{(1-2 n)}+\frac{\phi^{2}}{(1+n)}\right]
$$


For turbulent regime, $f$ is based on the correlations described by Metzner and Reed (1955) and Dodge and Metzner (1959), given by:

$$
\frac{1}{\sqrt{f}}=\frac{4}{n^{0.75}} \log \left[R e^{\prime} f^{(2-n) / 2}\right]-\frac{0.4}{n^{1.2}}
$$

Figure 4.19 shows that experimental friction factors obtained and the correlations stated by Malin (1998). The experimental data for fluid $10 \mathrm{~W}$ follows the behavior described by the correlation for $n=1$ and $H e_{H B}=0.1$, which for laminar $R e_{D}^{\prime}$ has a similar behavior for Newtonian fluids, a $n$ value of 0.9 is the responsible for this like Newtonian behavior for laminar conditions. Moreover, for $\operatorname{Re}_{D}^{\prime}$ turbulent values the relationship with $f$ is lower than for Newtonian fluids. The presence of the yield stress and the shear-thinning behavior affects the behavior of the fluid substantially. For fluids $20 \mathrm{~W}, 30 \mathrm{~W}$, and $40 \mathrm{~W}$ the experimental values reach the correlation for $n=0.6$ and $H e_{H B}=1$. Even though the $H e_{H B}$ value calculated for fluids $30 \mathrm{~W}$ and $40 \mathrm{~W}$ is close to 10 , the experimental data seems to be more influenced by $n$; thus, the experimental values are close to the correlation for $n=0.6$. A maximum error of $68 \%$ was obtained for the fluid $40 \mathrm{~W}$ at the lowest value, $R e_{D}^{\prime}=1$. For low Reynolds number, the accuracy of the measurements are affected due to the low flow rate imposed through the flow loop. An average error of $22 \%$ was obtained for all the experimental data obtained for $f$, which means a good agreement with the data in the literature, and the validation of the experimental setup.

Figure 4.19 - Experimental friction factor coefficients obtained for a circular section for each viscoplastic solutions. Experimental data are compared with the correlations of Malin (1998) for viscoplastic fluids, and Blasius (1913) correlation for Newtonian fluids.

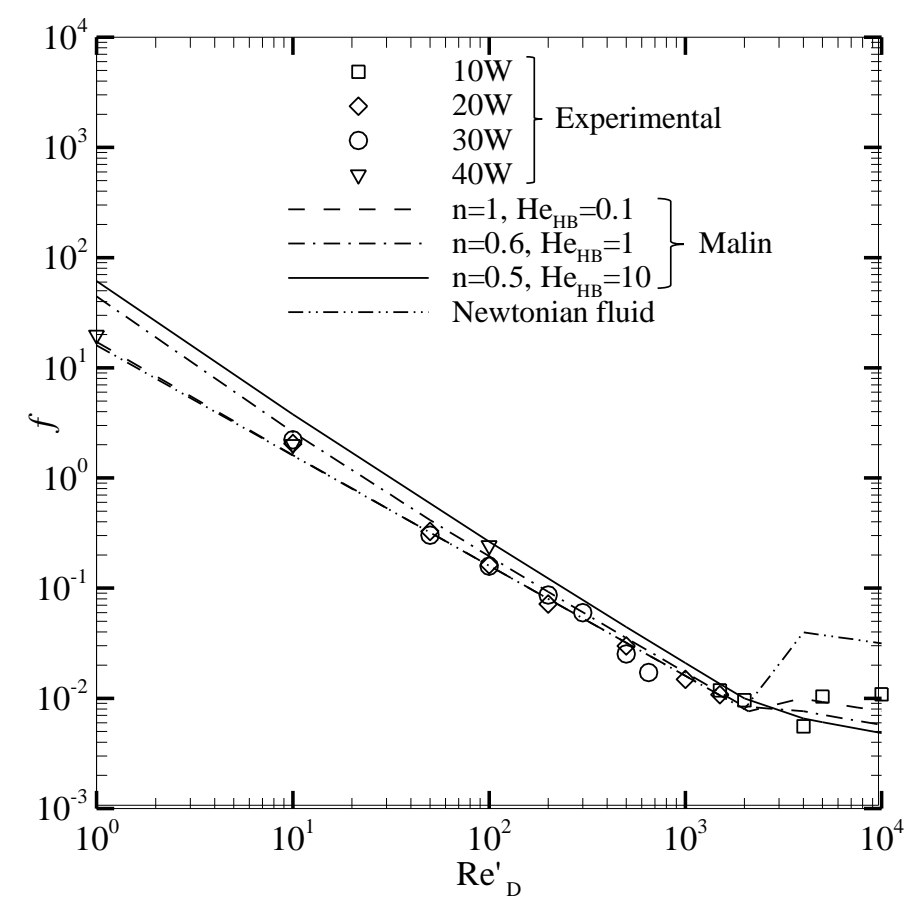

Source: Author (2019).

It is also possible to state that the friction factor increases for low $n$ values at the laminar regime. Once the turbulent regime is reached the friction factor is lower as compared to Newtonian fluid correlations. So, the shear-thinning index behavior is more relevant than the $H e_{H B}$ for Herschel-Bulkley fluids, as the 
curves for $H e_{H B}=1$ and $H e_{H B}=10$ are similar as the slope in laminar conditions (HANKS, 1963; HANKS et al., 1967; MALIN, 1998). Also, it is possible to appreciate that all the fluids had a common transitional regime between $2100<R e_{D}^{\prime}<4000$, according to the correlation stated by Malin (1998). This is owing to for similar $n$, there is a sensitive behavior of $R e^{\prime}$ respect to $H e_{H B}$ for values larger than 50 . 


\section{RESULTS AND DISCUSSIONS}

This chapter presents the results of the flow behavior of four viscoplastic ultrasound gel solutions at different regimes through an axisymmetric contraction with a ratio $\beta=1.85$.

The first section shows the pressure profiles behavior along the flow-loop, near to the entrance and the exit region at the contraction test section for different flow regimes. The pressure loss and the coefficient of pressure loss are calculated and compared with data from the literature. Following, the axial and radial velocity profiles are obtained from the mean vectorial field calculated by PIV technique. Then, the flow behavior is analyzed based on the vortex structures and unyielded regions observed at the test section. Finally, an effective diameter at the entrance of the contraction is presented and a correlation between the Reynolds numbers at the test section entrance and the contraction plane is presented.

\subsection{PRESSURE LOSSES}

In this section the static pressure in five points along the flow-loop is presented. Then, using the static pressure values, the pressure drop at the contraction is extrapolated, and the behavior of the pressure drop coefficient is presented.

\subsubsection{Pressure Profiles}

The pressure along the test section was measured according to the procedure described in section 4.7. Experimental results show that the pressure along the axial direction of the test section decreases linearly. This linear trend allowed to fit the pressure gradient by a linear equation, using the least squares method.

The pressure gradients are shown at Fig.5.1, linear equations for the upstream and downstream regions were extrapolated until the contraction position $(Z / D=0)$ to calculate $\Delta P_{c o n}$. The linear correlations have good agreement with the experimental data obtained. The square value for the correlation coefficients was calculated $\left(R^{2}\right)$, obtaining a range between 1.00 and $9.91 \times 10^{-1}$ and an average value of $9.99 \times 10^{-1}$ for the upstream region of the contraction and 1.00 for the downstream one. Pressure values are presented in Pascal (Pa) through the dimensionless axial distance from the contraction. The non-dimensionalization for the axial distance was done with the test section entrance diameter $(D)$, and are presented as a function of the Reynolds number defined by Eq. (2.45) (MALIN, 1998), in order to compare the data with correlations found in the literature.

From Fig. 5.1, it is possible to state that as higher $R e_{D}^{\prime}$ are reached, higher pressures in the flow-loop are required. The pressure losses at the downstream region decreases thus the slope between points 4 and 5 also decreases for each solution. As the concentration of ultrasound gel is increased, the pressure required for flowing is higher, thus as greater is the $\tau_{0}^{H}$ value more pressure will be required to counter the viscous resistance. 
Figure 5.1 - Pressure profiles along the flow-loop as a function of the dimensionless distance from the contraction $Z / D$ and the Reynolds number at the test section entrance, $R e_{D}^{\prime}$. Each viscoplastic solution is presented: (a) $\tau_{0}^{H}=0.07 \mathrm{~Pa}(10 \mathrm{~W})$, (b) $\tau_{0}^{H}=0.26 \mathrm{~Pa}(20 \mathrm{~W})$, (c) $\tau_{0}^{H}=2.82 \mathrm{~Pa}(30 \mathrm{~W})$ and (d) $\tau_{0}^{H}=6.99 \mathrm{~Pa}(40 \mathrm{~W})$.
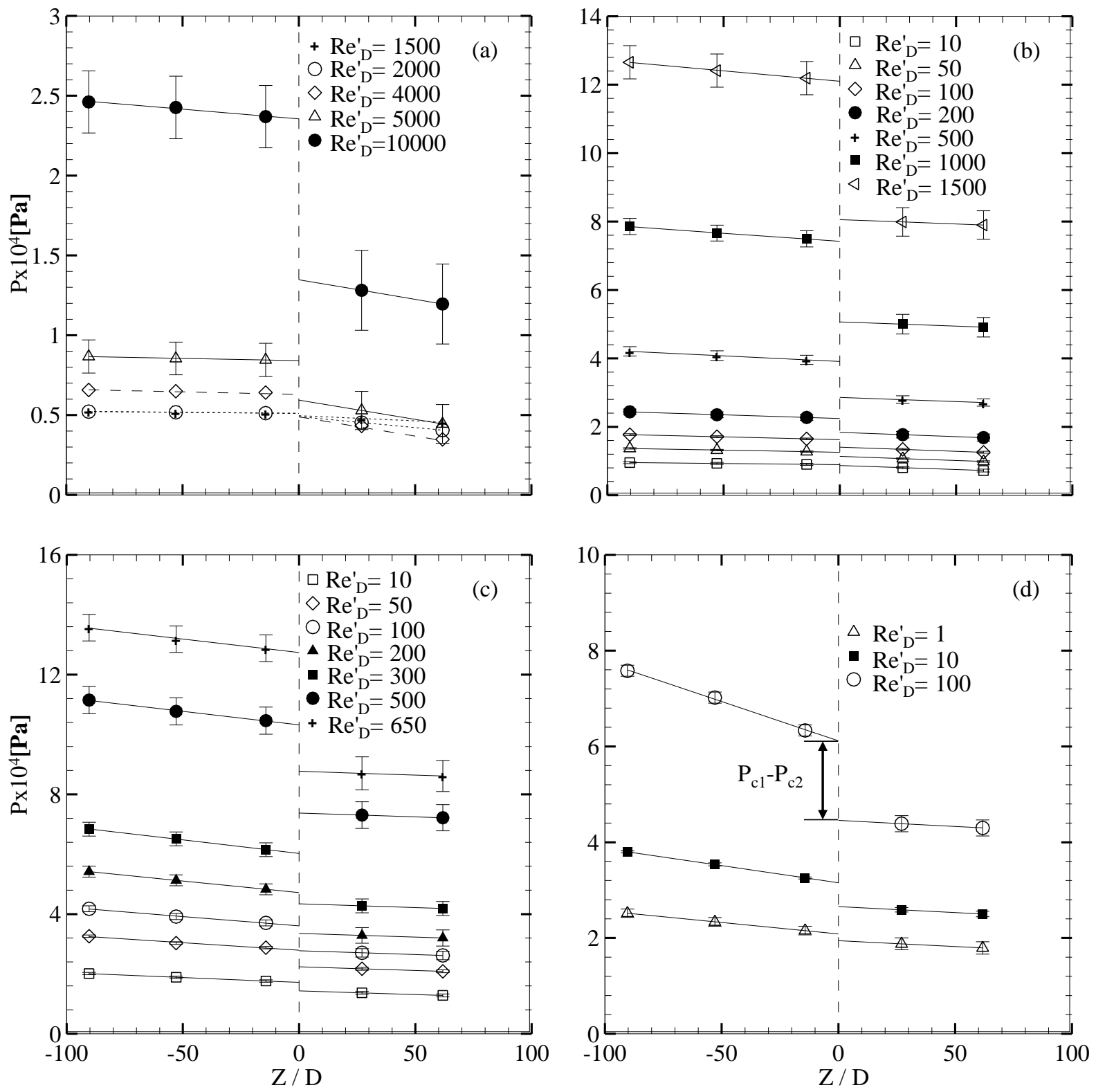

Source: Author (2019).

For lowest concentration, which presents the lowest $\tau_{0}^{H}$ value a particular behavior is noticed, for laminar $R e_{D}^{\prime}$ the pressure profile at the downstream region does not follow a monotonic behavior. $R e_{D}^{\prime}=1500$ presents higher values along the test section than those for $\operatorname{Re}_{D}^{\prime}=4000$. This behavior can be explained by the low consistency coefficient of $10 \mathrm{~W}$ solution. Low pump rpm were set to guarantee the laminar and the transitional flow, leading to slight fluctuations on the pressure for low flow rate and low viscosities, increasing the pressure measurements uncertainty at the downstream region. Error bars for this solution has greater values than other solutions, that means a greater standard deviation of the 
experimental pressure data obtained, as presented in appendix B . Nevertheless, the experimental data presents coherent behavior according with the information in the literature (ISAYEV; HUANG, 1993).

\subsubsection{Pressure Drop at the Contraction}

As it was explained in section 4.7, the pressure drop at the contraction plane $\left(\Delta P_{c o n}\right)$ was obtained by extrapolating the pressure profile at the downstream and upstream region to the position of the contraction $(Z / D=0)$. The pressures $P_{c 1}$ and $P_{c 2}$ are the locations where the extrapolation of the pressure profiles cross the contraction plane, as showed in Fig. 5.1d. Then, using Eq. (4.27) $\Delta P_{\text {con }}$ is calculated.

For each viscoplastic solution, the $\Delta P_{\text {con }}$ was obtained and plotted as a function of $\operatorname{Re}_{D}^{\prime}$. Figure 5.2 shows that as greater is the $R e_{D}^{\prime}$ imposed, the greater will be $\Delta P_{\text {con }}$. Also, high $\tau_{0}^{H}$ leads to higher values for $\Delta P_{\text {con }}$. Such behavior is associated with the rheological properties of the fluids, as higher is $\tau_{0}^{H}$ and $m$ values, it is required greater mean velocities to reach high Reynold numbers, this leads to larger pressures values at the entrance of the test section and high energy losses, and consequently larger pressures drops at the contraction.

Figure 5.2 - Pressure drop for each yield stress solution as a function of the Reynolds number at the entrance section.

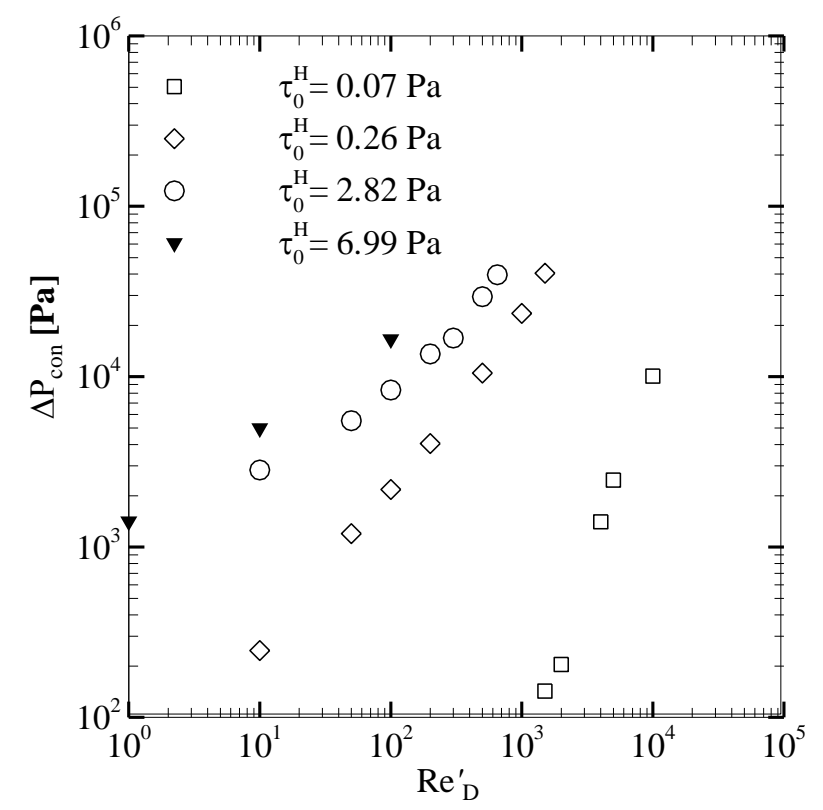

Source: Author (2019).

In order to compare the results obtained for viscoplastic fluid with some data from the literature for Newtonian fluid flow, $\Delta P_{\text {con }}$ was non-dimensionalized by the dynamic pressure at the entrance section, $\rho \bar{U}^{2}$. So, the dimensionless pressure is defined as the ratio between the static and dynamic pressure, where the dynamic pressure is the kinetic energy per unit volume of the fluid (PRITCHARD; MITCHELL, 2016). Figure 5.3 presents the results obtained for the dimensionless pressure drop at the contraction, given by:

$$
\Delta P_{c o n}^{\prime}=\frac{\Delta P_{c o n}}{\rho \bar{U}_{1}^{2}}
$$

where $\bar{U}_{1}$ is the mean velocity at the entrance of the test section. 
Figure 5.3 - Pressure drop non-dimensionalized by the dynamic pressure for each viscoplastic solution characterized by $H e_{H B}$ as function of $\operatorname{Re}_{D}^{\prime}$. Comparison with experimental studies performed for Newtonian fluid.

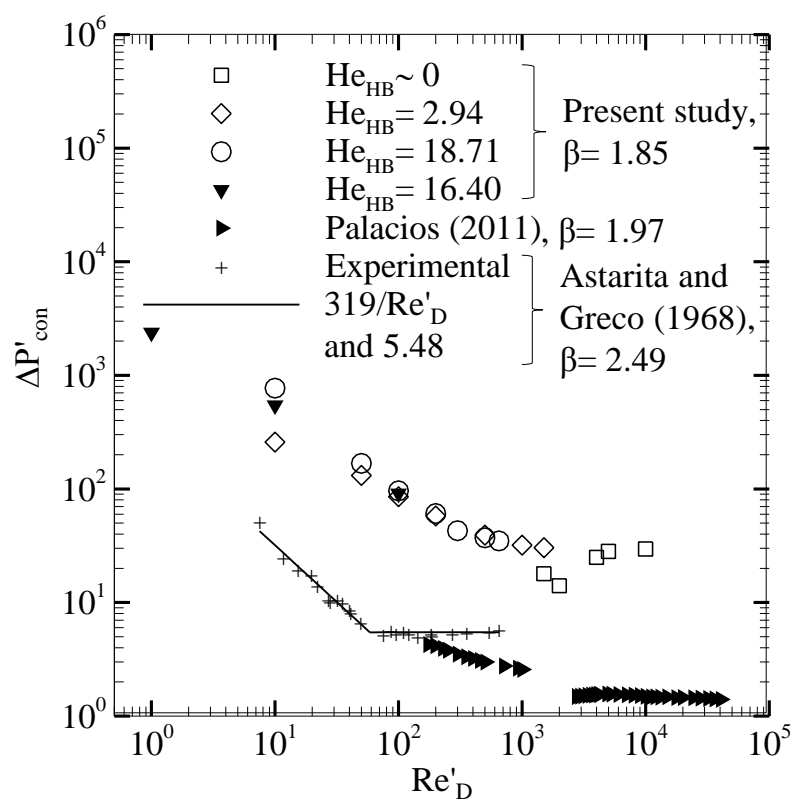

Source: Author (2019).

Experimental studies performed by Astarita and Greco (1968) and Palacios (2011) showed a powerlaw dependence of the pressure drop the contraction with the Reynolds number until a critical value is achieved. Above this critical value, the dimensionless pressure drop behavior describes a constant value. Astarita and Greco (1968) observed this critical Reynolds number at the laminar regime for $R e_{D}^{\prime}=58$, where the dimensionless pressure drop at the contraction reached a constant value of 5.48, see Fig. 5.3. Below this value, the correlation is given by $319 / R e_{D}^{\prime}$. This experimental study was performed just for laminar Reynolds numbers. On the other hand, Palacios (2011) found a different behavior for $\Delta P_{c o n}^{\prime}$. A decreasing correlation is presented for $R e_{D}^{\prime}$ between 150 and 1000 , with values between 4.29 and 2.58 for $\Delta P_{c o n}^{\prime}$. At the transitional and turbulent regimes, $\Delta P_{c o n}^{\prime}$ values are constant with a mean value of 1.51. The difference between these results is defined by the geometrical parameters and the flow-loop configuration. Nevertheless, there is a coherent behavior as it was expected by the authors.

In the present study for non-Newtonian fluids, higher pressure losses at the contraction than those for Newtonian fluids were found. At the laminar regime, there is a decreasing behavior until the transitional condition is reached, between $1 \leq R e_{D}^{\prime} \leq 2000$. Then, at the turbulent regime, there is a constant behavior that tends to a constant value of 29 , this is a higher value than the values obtained for Newtonian fluids flow through abrupt contractions by Astarita and Greco (1968) and Palacios (2011). This behavior is related to the presence of yield stress; thus, higher pressures are required to start the flow, which implies greater values for pressure along the test section and higher pressure drop at the contraction.

The viscoplastic solutions are described by the $\mathrm{He}_{H B}$ in order to show the dimensionless pressure drop as a function of the rheological properties. For the solution with $H e_{H B} \approx 0$, it is noted that a critical $R e_{D}^{\prime}$ near to the transitional regime is depicted, as presented in the friction factor correlation. This critical $R e_{D}^{\prime}$ is near to the value found by Fredy $\left(1500<R e_{D}^{\prime}<2400\right)$, nevertheless the viscoplastic fluids 
reach higher values when compared with Newtonian fluids, but there is not remarkable difference between them in the $\Delta P_{c o n}^{\prime}$ values. This behavior can be explained by the high $H e_{H B}$ values, thus high dynamic pressures are required for flowing the fluids and also high static pressure are noticed as the fluid is more consistence, and similar $\Delta P_{c o n}^{\prime}$ ratios are reached.

\subsubsection{Pressure Loss Coefficient}

The pressure loss coefficient is calculated based on the results obtained for $\Delta P_{c o n}$. As it was explained at section $2.2, k_{\text {con }}$ is calculated with the Eq.(2.51). Repeated here for convenience,

$$
k_{\text {con }}=\frac{\left(\Delta P_{\text {con }} / \rho g\right)+\left(\alpha_{1} \bar{U}_{1}^{2}-\alpha_{2} \bar{U}_{2}^{2}\right) / 2 g}{\bar{U}_{2}^{2} / 2 g}
$$

where $\alpha_{1}, \alpha_{2}, \bar{U}_{1}$ and $\bar{U}_{2}$ are the kinetic energy correction factor and the mean velocity at the downstream and upstream region of the contraction. The values for $\alpha_{1}$ and $\alpha_{2}$ were calculated with the correlations used by Mika (2011), where the criteria of Strzelecka and Jezowiecka-Kabsch (2008), described in section 2.2, are used for viscoplastic fluid flow in turbulent regime. For the laminar regime, $\alpha_{1}$ and $\alpha_{2}$ were calculated using the Eq. (2.52), which correlates the kinetic energy correction factor with the yield stress of the viscoplastic fluid. Figure 5.4 shows that for laminar $R e_{D}^{\prime}$, the pressure loss coefficient presents a power-law decreasing. For $R e_{D}^{\prime}$ above $3000, k_{\text {con }}$ depicts a constant behavior. This is noted for $10 \mathrm{~W}$ and $20 \mathrm{~W}$ solutions, which were led to transitional and turbulent $R e_{D}^{\prime}$.

In the literature, there are mainly two equations to fit the pressure loss coefficient curves. These equations are used independently of the kind of fitting considered (KFURI et al., 2011). The one presented by Kittredge and Rowley (1957) is given by:

$$
k=C R e^{-D}
$$

where $C$ and $D$ are constants. And the two $K^{\prime} s$ method presented by Hooper (1981):

$$
k=\frac{k_{1}}{R e}+k_{\infty}
$$

where $k_{1}$ is the first part proportional to the inverse Reynolds number and it is related to the laminar regime, while the value $k_{\infty}$ is the constant level associated with the turbulent regime.

For the present study the method proposed by Hooper (1981) was used, whose advantage is the simplicity due to the fact it has two parameters that can represent both flow regimes. The fit of the experimental data by the K's Method is also presented in Fig.5.4 by a solid black line and the tends for laminar and turbulent regime are depicted by dashed and dot-dashed lines.

After fit the experimental values for $k_{\text {con }}$ by the $K^{\prime} s$ method values for $k_{1}=338$ and $k_{\infty}=1.38$ were obtained. Figure 5.5 depicts the trends for $K^{\prime} s$ fit, and they are compared with the correlations of Turian et al. (1998), Fester et al. (2008), and Mika (2011) for $\beta=2$ contractions. The correlations parameter were summarized in Table 3.1, where for a laminar regime Turian et al. (1998) stated a correlation $900 / R e_{D}^{\prime}$ and Fester of $288 / R e_{D}^{\prime}$. Nevertheless, Mika (2011) found a power-law correlation give by $11000 / R e_{D}^{\prime 1.26}$. For the turbulent regime, each correlation reaches different constants values, 1.114 for Turian et al. (1998) 
Figure 5.4 - Pressure loss coefficient at the contraction in function of the Reynolds number at the entrance section. Experimental values were fit by K's method proposed by Hooper (1981). The laminar and turbulent trends are presented by dashed and dot-dashed lines.

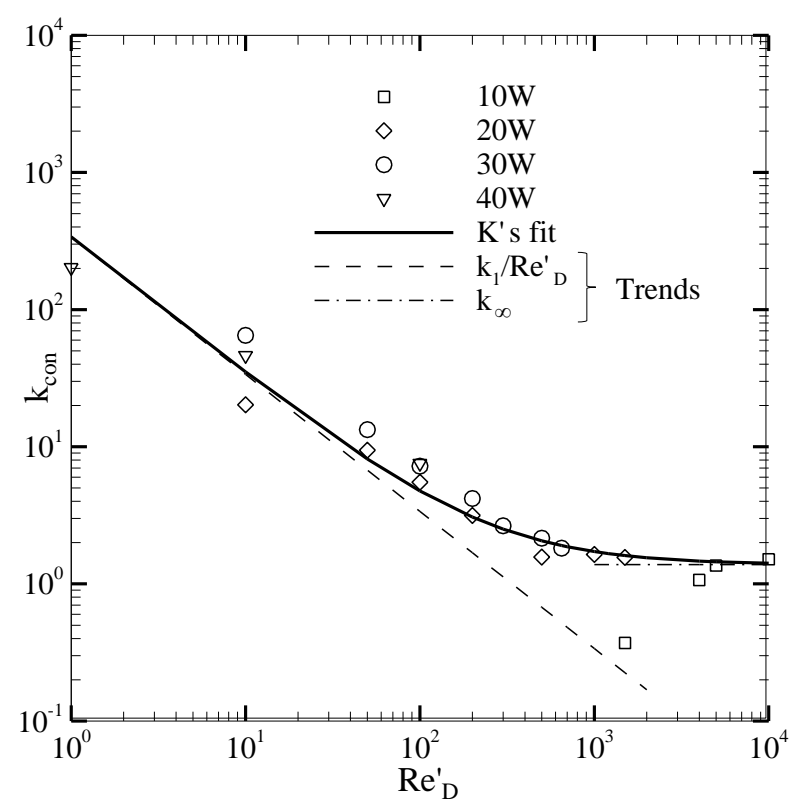

Source: Author (2019).

and 0.38 for Mika (2011)'s correlation. Then, for the present study a correlation for the pressure loss coefficient at the contraction is given by the following expression:

$$
k_{\text {con }}=\frac{338}{R e_{D}^{\prime}}+1.38
$$

As the different correlations reach different values and expressions for laminar and turbulent regimes, it is possible to state that these correlations depend on the configuration of the flow-loop and the contraction, thus the experimental studies found in the literature used polymer solutions and some composed fluids that are fitted by the different models for viscoplastic fluids. Also, the experimental studies were performed in different experimental setup configurations, then, it is possible to conclude that pressure losses, pressure drop and the pressure loss coefficient at the contraction are sensitive to the configuration and the geometrical variations along the flow-loop.

Nevertheless, it is remarkable that the power-law behavior of the pressure loss coefficient for laminar regimes $R e_{D}^{\prime}<2000$ is near to the Fester et al. (2008) correlation, and the constant value for $R e_{D}^{\prime}>4000$, it is similar to the asymptote found by Turian et al. (1998), showing a good agreement with the data found in the literature. And, It is highlighted that as the pressure loss coefficient and the dimensionless pressure drop has similar behavior, after the turbulent regime is reached.

Finally, it is possible to fit the viscoplastic fluids in a same correlation for laminar regime, as stated by the correlations there is no a dependence on the rheological parameters for the pressure loss coefficient, but in the $R e_{D}^{\prime}$; meanwhile, for turbulent conditions a constant value is depicted. 
Figure 5.5 - Comparison of the pressure loss coefficient correlations found in the present study with the correlations found in the literature for viscoplastic fluid flow.

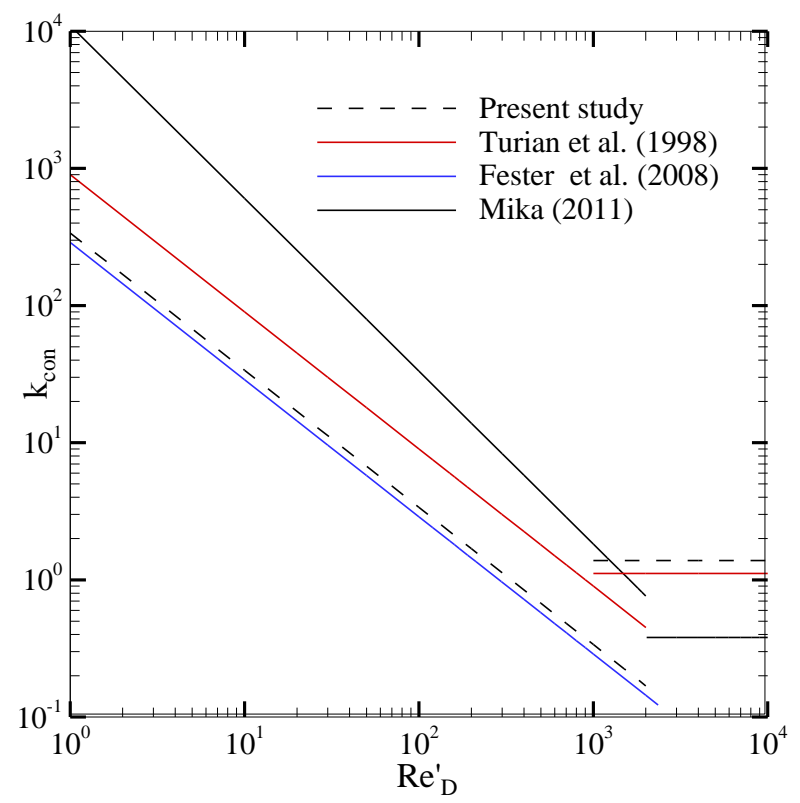

Source: Author (2019).

\subsection{FLOW FIELD AND MEAN QUANTITIES}

In this subsection the data obtained using the PIV technique are presented. The flow vector field and the derived quantities as velocity profile, streamlines and turbulent quantities obtained at the meridional plane of the contraction. The values obtained are non-dimensionalized by the mean velocity at the test section entrance $(\bar{U})$ and the diameter at the entrance section $(D)$. As it was expressed in the last subsection, the values for the Reynolds numbers are set based on the entrance diameter, $R e_{D}^{\prime}$.

\subsubsection{Mean Velocity Vector Map}

The mean velocity vector map was obtained using the features of the Dynamic Studio® software described in subsection 4.7.2. A correlation of the seeding particles displacement allows to calculate the vector for each position and then a statistic from multiple vector maps is done to obtain a single vector map of the mean velocity vectors. Other statistical quantities are calculated, as well, including mean velocities, standard deviations, and fluctuations for the axial and radial velocity components.

\subsubsection{Laminar flow}

The laminar flow statistics were obtained for a total of twelve $R e_{D}^{\prime}$, distributed in all viscoplastic solutions: 10W, 20W, 30W, and 40W. Table 5.1 presents all the parameters set to obtain the mean statistic velocity vector map for these laminar Reynolds numbers.

After acquiring the images, it was calculated the arithmetic mean of the instantaneous vector fields for each position of the area visualized. Then, the mean statistic velocity field for radial $(v)$ and axial $(u)$ 
Table 5.1 - List of parameters set to visualize laminar fluid flow

\begin{tabular}{|c|c|c|}
\hline \multirow[t]{2}{*}{ Pipe } & Entrance diameter $[\mathrm{mm}]$ & 25.9 \\
\hline & Material & Acrylic \\
\hline \multirow{6}{*}{$\begin{array}{l}\text { Flow } \\
\text { properties }\end{array}$} & Fluid & $10 \mathrm{~W} / 20 \mathrm{~W} / 30 \mathrm{~W} / 40 \mathrm{~W}$ \\
\hline & Density $\left[\mathrm{Kg} / \mathrm{m}^{3}\right]$ & 1018 / 1002 / 1014 / 1038 \\
\hline & Yield stress $[\mathrm{Pa}]$ & 0.07 / 0.26 / 2.82 / 6.99 \\
\hline & Consistency coefficient $\left[\mathrm{Pa} \cdot \mathrm{s}^{n}\right]$ & $0.002 / 0.25 / 0.85 / 1.98$ \\
\hline & Flow behavior index [-] & $0.99 / 0.63 / 0.55 / 0.49$ \\
\hline & $R e_{D}^{\prime}$ & $\begin{array}{l}1500 ; 2000 / 10 ; 100 ; 500 ; 1500 / \\
10 ; 100 ; 500 / 1 ; 10 ; 100\end{array}$ \\
\hline \multirow{3}{*}{$\begin{array}{l}\text { Particles } \\
\text { properties }\end{array}$} & Type & Spherical PSP-Rhodamine B \\
\hline & Density $\left[\mathrm{Kg} / \mathrm{m}^{3}\right]$ & 1150 \\
\hline & Average diameter $[\mu \mathrm{m}]$ & $5-10$ \\
\hline \multirow{4}{*}{$\begin{array}{l}\text { Laser } \\
\text { settings }\end{array}$} & Type & Double- Nd:YG \\
\hline & Wavelength $[\mathrm{nm}]$ & 532 \\
\hline & Energy $[\mathrm{mJ}]$ & 60 \\
\hline & Laser beam thick $[\mathrm{mm}]$ & 1 \\
\hline \multirow{3}{*}{$\begin{array}{l}\text { Camera } \\
\text { settings }\end{array}$} & Type & CMOS \\
\hline & Resolution [Pixels] & $2352 \times 1728$ \\
\hline & Trigger rate $[\mathrm{Hz}]$ & 50 \\
\hline \multirow[t]{4}{*}{ Image } & f-number & 8 \\
\hline & Visualization area $[\mathrm{mm} \times \mathrm{mm}]$ & $52 \times 26$ \\
\hline & Time between pulses $[\mu \mathrm{s}]$ & $\begin{array}{l}600 ; 450 / 2000 ; 600 ; 350 ; 106 ; 50 / \\
900 ; 190 ; 65 / 2500 ; 600 ; 128\end{array}$ \\
\hline & Number of images & 1040 \\
\hline \multirow[t]{5}{*}{ PIV analysis } & Type of correlation & Central difference \\
\hline & Validation & Moving average \\
\hline & Interrogation area & $32 \times 32$ \\
\hline & Overlap & $50 \%$ \\
\hline & Vectors per image & $146 \times 107=15622$ \\
\hline
\end{tabular}

Source: Author (2019).

components were given by:

$$
\begin{aligned}
& u(r, z)=\frac{1}{N} \sum_{i=1}^{N} u_{i}(r, z) \\
& v(r, z)=\frac{1}{N} \sum_{i=1}^{N} v_{i}(r, z)
\end{aligned}
$$

where $N$ is the number of instantaneous vector field measured, and $r$ and $z$ are the radial and axial coordinates, respectively.

Figure 5.6 shows the mean flow vector field for the laminar regime of $30 \mathrm{~W}$ solution at $R e_{D}^{\prime}=10$. It is possible to observe the vector field generated by correlation of the images obtained with the PIV 
technique. behavior of the. As the velocity values are nondimensionalized by $\bar{U}$, it is possible to analyze the dimensionless velocity $(u / \bar{U})$. When $Z / D=2$, far from the contractions effects, $u / \bar{U}$ is equal to 1.35 at the centerline. This value is very close to the experimental data presented by Peixinho et al. (2005) where the relation for the dimensionless velocity is near to 1.4 for laminar regimes.

Figure 5.6 - Mean statistic velocity vector field for laminar flow. Solution $30 \mathrm{~W} R e_{D}^{\prime}=10$.

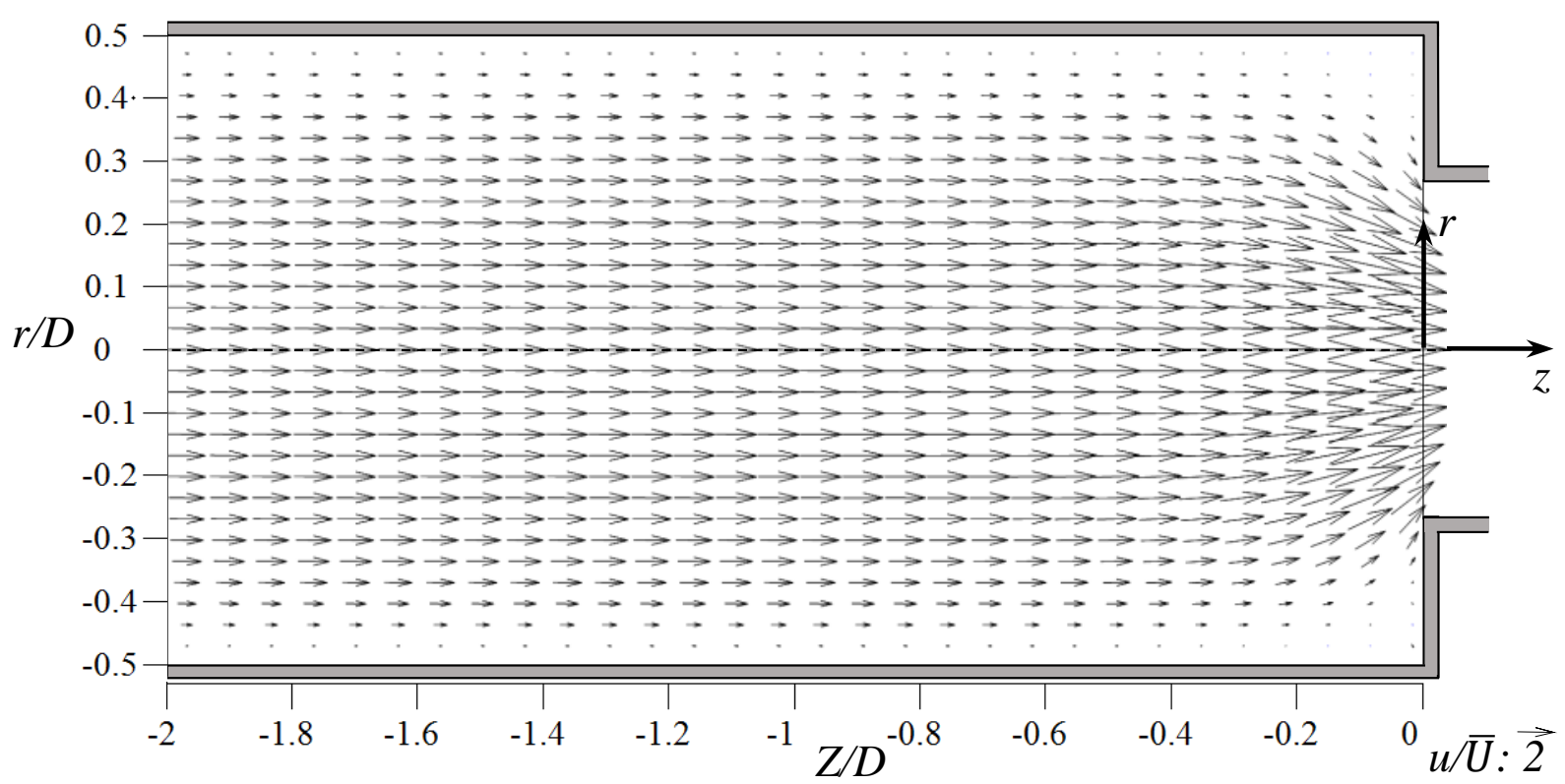

Source: Author (2019).

Near to the contraction, $u / \bar{U}$ reaches values near to 2.5 , due to the flow acceleration at the region $-0.2<Z / D<0$. As presented in Fig 5.6, it is possible to compare using the scale relation for the vector size. It is important to highlight that the vector field has been increased 30 times the real size, and there is just $1 / 8$ part of the total vector generated after the data processing.

The scalar map for the axial and radial velocities were obtained for all the $R e_{D}^{\prime}$ in the laminar regime using the mean vector field. In order to facilitate the analysis of the data presented in this study, the axial and radial velocity maps are separated in this subsection.

Figure 5.7 presents the laminar dimensionless velocity in the axial direction for $10 \mathrm{~W}$ and $20 \mathrm{~W}$ fluids. The values are presented among a bar-colored scale with limits in -0.5 and 3.5. The $R e_{D}^{\prime}$ values presented for fluid $10 \mathrm{~W}$ are near to the transitional regime. The $u / \bar{U}$ ratio near to the entrance of contraction reaches a value of 3 . Instead, for fluid $20 \mathrm{~W}$ at low $R e_{D}^{\prime}$ values, $u / \bar{U}$ ratio reaches values of 4 at the entrance of the contraction. So, as the $R e_{D}^{\prime}$ increases, the $u / \bar{U}$ ratio decreases in this region. This behavior is related to the reduction of the central plug region of the viscoplastic fluid flow, due to the increasing of the velocity gradients.

Furthermore, at $R e_{D}^{\prime}=1500$ for $20 \mathrm{~W}$ fluid, it is possible to observe an asymmetric behavior of the axial velocity near to the entrance of the contraction. This asymmetric behavior suggests a link with the instability created by the appearance of turbulent spots for $\operatorname{Re}_{D}^{\prime}$ near to the transition regime. This behavior with asymmetric condition was also observed by Escudier and Presti (1996) and Peixinho et al. 
Figure 5.7 - Mean axial velocity map for laminar $R e_{D}^{\prime}$. a) and b) present the flow maps for 10W fluid. c), d), e), and f) show the dimensionless axial velocity maps for $20 \mathrm{~W}$ fluid.
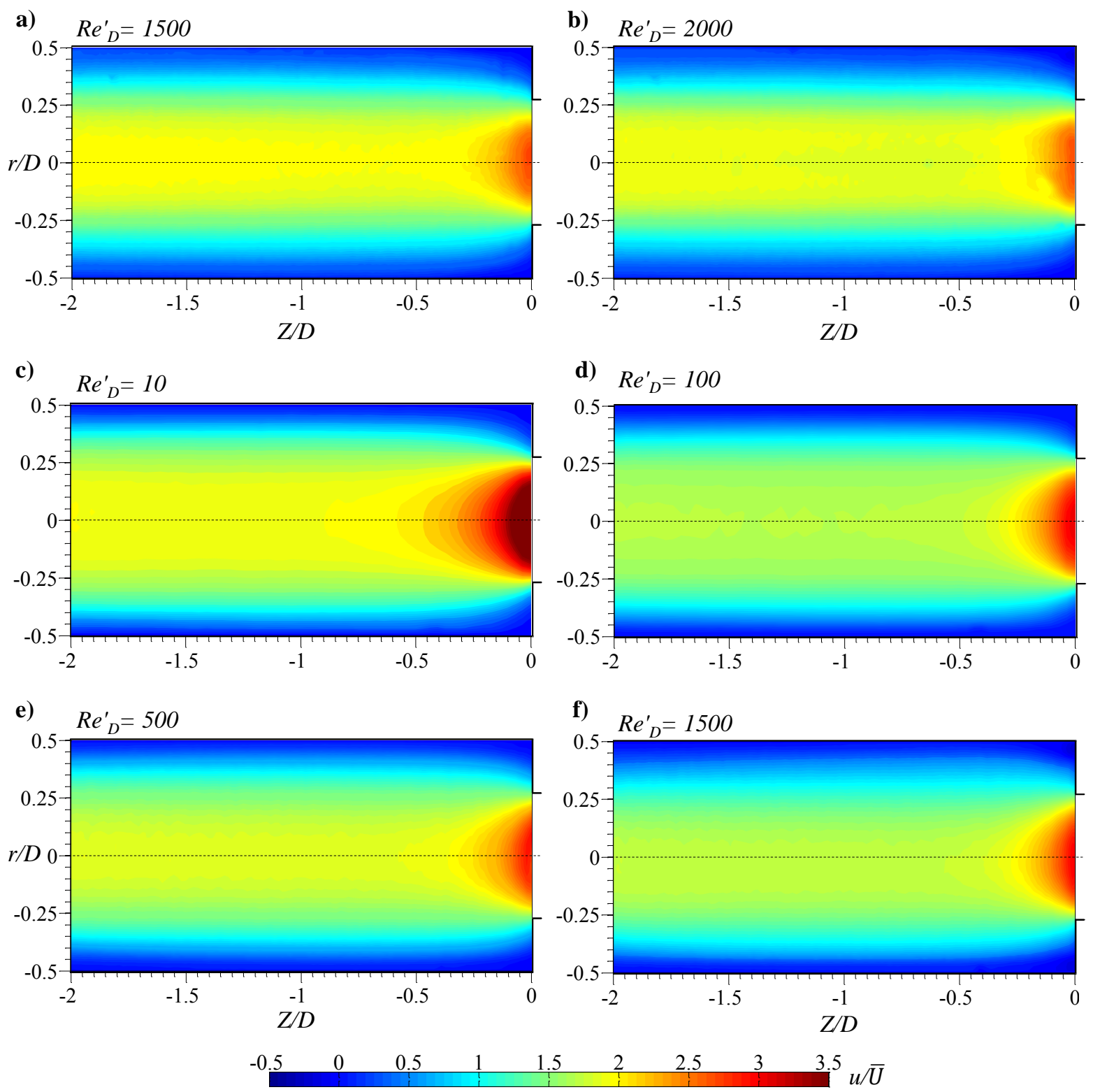

Source: Author (2019).

(2005) for a thixotropic and viscoplastic fluid, respectively. This topic is explained in detail further in the present study.

The axial velocity maps for $30 \mathrm{~W}$ and $40 \mathrm{~W}$ fluids are presented in Fig. 5.8. The axial velocity for $30 \mathrm{~W}$ and $40 \mathrm{~W}$ fluids present a decrease of the dimensionless velocity at the contraction entrance as the $R e_{D}^{\prime}$ increases. For low $R e_{D}^{\prime}$ at the contraction plane $u / \bar{U}$ reaches values of 3.5 , such is the case of a $R e_{D}^{\prime}=10$ for $30 \mathrm{~W}$ and $40 \mathrm{~W}$ fluids. The $u / \bar{U}$ value decreases with the increase of the $R e_{D}^{\prime}$ at the laminar regime.

For $30 \mathrm{~W}$ and $40 \mathrm{~W}$ fluids, the central plug core region presents low $u / \bar{U}$ values between 1 and 1.5 for low $R e_{D}^{\prime}$. The ratio velocity increases with the $R e_{D}^{\prime}$, leading to $u / \bar{U}$ values of 2 for $R e_{D}^{\prime}$ between 100 
Figure 5.8 - Mean axial velocity map for laminar $R e_{D}^{\prime}$. a), b), and c) show the maps for 30W fluid. d), e), and f) present the maps for $40 \mathrm{~W}$ fluid.
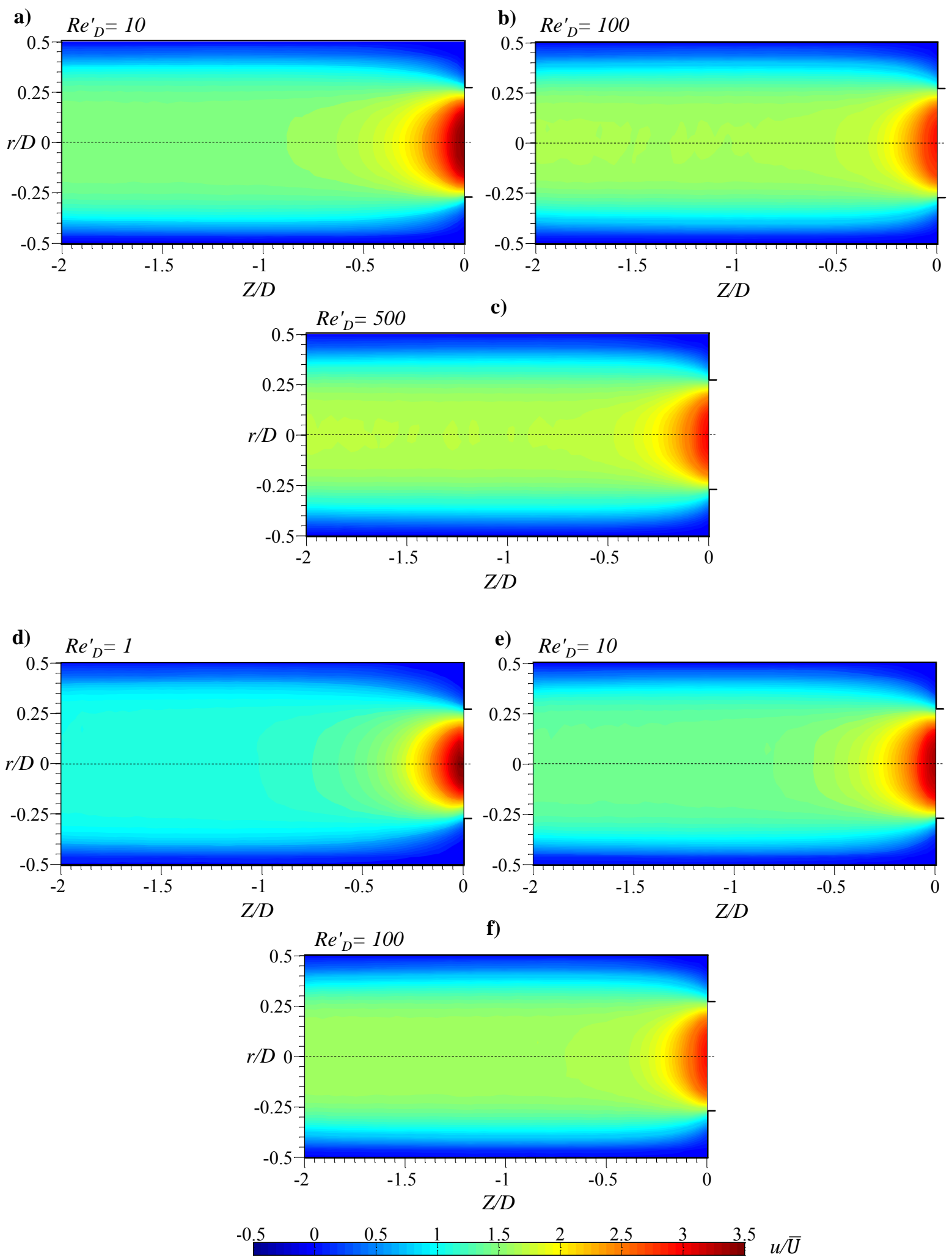

Source: Author (2019). 
and 500. Nevertheless, this increment of the $u / \bar{U}$ values is presented just for low $\operatorname{Re}_{D}^{\prime}$ and for viscoplastic fluids with higher $m$, this is explained below in the centerline velocity analysis. For $10 \mathrm{~W}$ and $20 \mathrm{~W}$ fluids, the values for $u / \bar{U}$ are higher at $R e_{D}^{\prime}$ near to the transitional regime.

For Newtonian fluids, Durst and Loy (1985), and Palacios (2011) observed axial dimensionless velocity ratios with values near to 4 at the entrance of the contraction for low laminar Reynolds numbers. For high Reynolds, $u / \bar{U}$ decreases near to 3.8 , which is a quite small variation among the laminar regime Reynolds numbers. For $R e_{D}^{\prime}$ near to the transitional regime, the flow maintains axial symmetry behavior, and a recirculation region is observed at the corner of the contraction leading to negative $u / \bar{U}$ values.

The dimensionless radial velocity $v / \bar{U}$ is presented in Fig. 5.9 and Fig. 5.10, values of $v / \bar{U} \approx 0$ are obtained far from the contraction plane. After $Z / D>-0.5$, the effects of the contractions lead to $v / \bar{U}$ ratio absolute values between 1 and 1.5. For low laminar $R e_{D}^{\prime}$, the $v / \bar{U}$ ratio achieves values of 1.5 , and a greater area is affected by the contractions, thus, for $20 \mathrm{~W}, 30 \mathrm{~W}$, and $40 \mathrm{~W}$ fluids are observed variations of the dimensionless radial velocity from $Z / D=-1$ to the contraction plane. As the $R e_{D}^{\prime}$ increases the area under the effect of the contraction is reduced, and the $v / \bar{U}$ ratio reaches maximum values between 0.8 and 1 at the contraction plane. According to the reference coordinate system used in this work, the radial velocity component has positive and negative values in the region adjacent to the contraction.

From Fig. 5.10, it is observed that for high $\tau_{0}^{H}$ values a reduction of the maximum $v / \bar{U}$ ratio value is observed. When compared the fluids $20 \mathrm{~W}, 30 \mathrm{~W}$, and $40 \mathrm{~W}$ for the same $R e_{D}^{\prime}=10$, a variation of the maximum value for the radial velocity ratio is visible. For fluid $20 \mathrm{~W}$, the ratio achieves absolute values near to 1.5 ; instead fluid $40 \mathrm{~W}$, which reaches a value of 0.9 . Also, fluid $40 \mathrm{~W}$ presents a particular behavior for a $R e_{D}^{\prime}=1$, the $v / \bar{U}$ presents a maximum absolute value of 0.6 .

It is possible to state that lowest $v / \bar{U}$ ratios at the contraction plane are observed for low $\operatorname{Re}_{D}^{\prime}$; then after a laminar $R e_{D}^{\prime}=10$ is reached, the $v / \bar{U}$ values increase. Also, $v / \bar{U}$ decrease at the contraction plane when $R e_{D}^{\prime}$, and $\tau_{0}^{H}$ values increase for the laminar regime.

Palacios (2011) observed $v / \bar{U}$ ratio values of 2.4 and 1.86 for Reynolds numbers of 185 and 1266 , respectively. Similarly to $u / \bar{U}$ ratios, for viscoplastic fluids the values for $v / \bar{U}$ are lower than for Newtonian fluids. So, a dependence of the radial and axial velocities on the rheological parameters is found, for high $\tau_{0}^{H}$ there are low ratios due to a greater plug core is presented, and a flat axial velocity profile is obtained, reducing the ratio values for $u / \bar{U}$, and $v / \bar{U}$.

\subsubsection{Turbulent flow}

Similar to that performed for the laminar regime, Table 5.2 presents the parameters used for the image acquisition for the turbulent regime.

For the turbulent regime, the mean velocity vector map was obtained just for the viscoplastic solution $10 \mathrm{~W}$. Because of the lowest consistency and yield stress, it was possible to reach the turbulent regime with this fluid. For the other solutions, it was not possible to guarantee good accuracy for pressure measurements and image acquisition. Nevertheless, it was possible to visualize and compare the flow evolution at the turbulent regime for viscoplastic fluid.

The mean statistic velocity map is calculated using the Equations (5.6) and (5.7), which were described 
Figure 5.9 - Mean radial velocity map for laminar $R e_{D}^{\prime}$. a) and b) are the flow maps obtained for $10 \mathrm{~W}$ fluid. c), d), e), and f) are the dimensionless radial velocity maps for $20 \mathrm{~W}$ fluid.
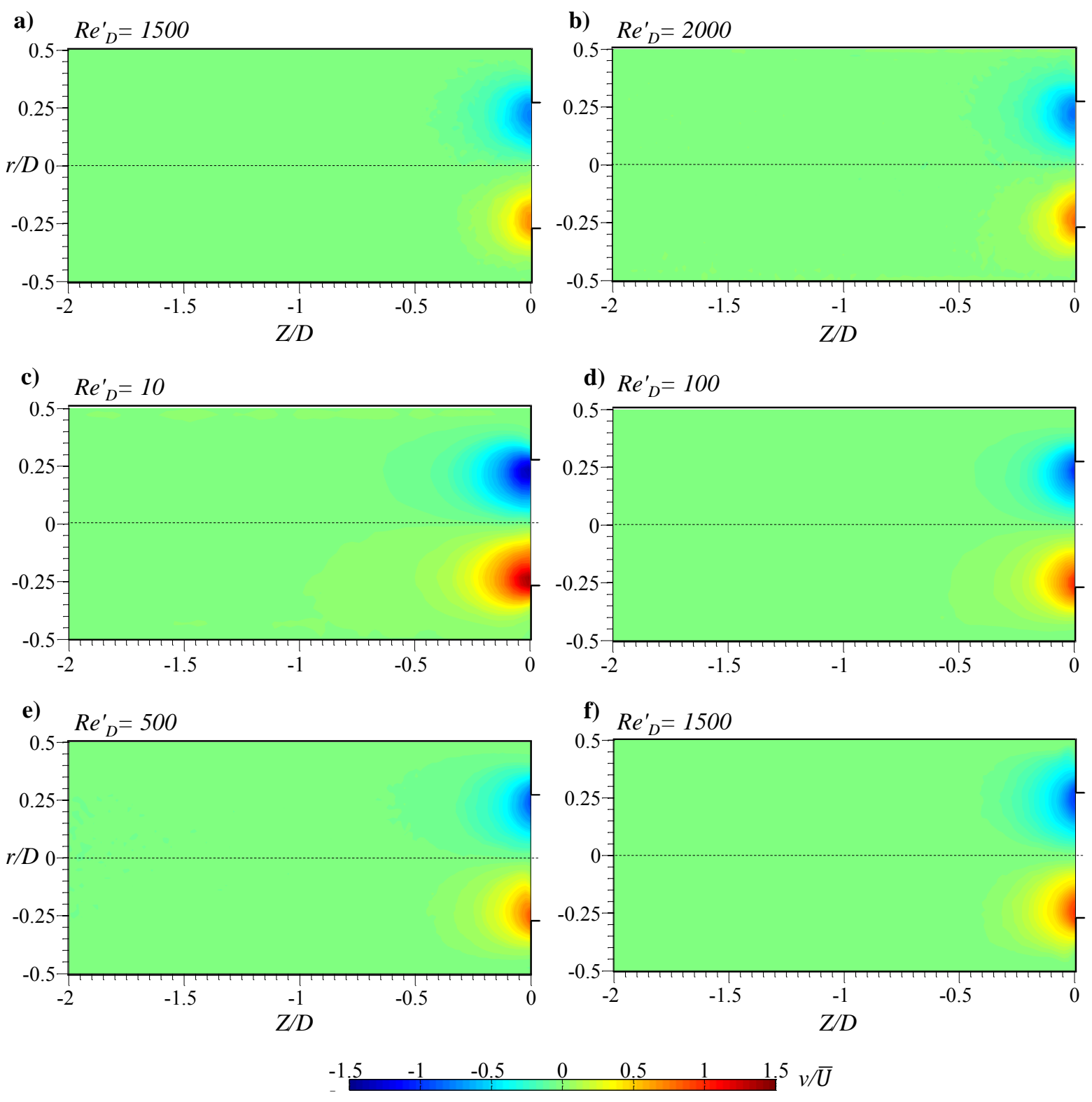

Source: Author (2019).

in the last subsection for laminar regime. Also, 1040 instantaneous maps are obtained from the image acquisition, then a statistic of the instantaneous maps is made to generate a mean vector map. The axial and radial components are presented in Figs. 5.11 and 5.12.

The axial dimensionless velocity for turbulent regime presents lower values than the laminar one. As observed for the laminar regime, the lowest $u / \bar{U}$ values are found at the entrance of the test section, where $Z / D=-2$. Near the contraction plane are the highest $u / \bar{U}$ values, which 2.8 is the maximum value for $R e_{D}^{\prime}=4000$, and 2.5 for $R e_{D}^{\prime}=10000$. So, as the $R e_{D}^{\prime}$ increases, the $u / \bar{U}$ values are reduced, and a uniform distribution is noted in the central region, where the plug core has completely vanished.

According to the experimental study of Peixinho et al. (2005), the viscoplastic fluids present a flat 
Figure 5.10 - Mean radial velocity distribution for laminar $R e_{D}^{\prime}$. a), b), and c) show the maps for 30W fluid. d), e), and f) present the maps for 40W fluid.
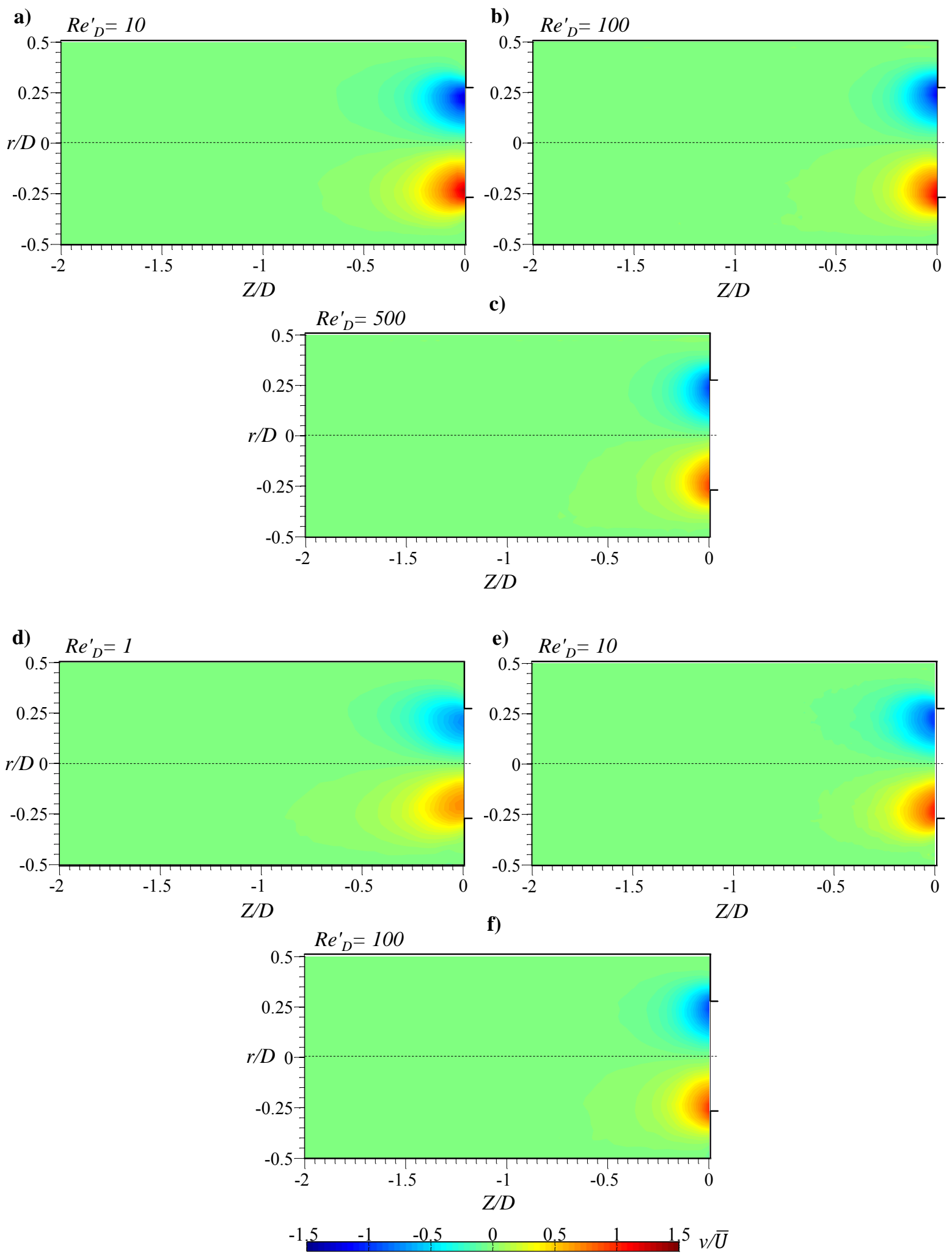

Source: Author (2019). 
Table 5.2 - Listing of parameters set to visualize turbulent fluid flow

\begin{tabular}{|c|c|c|}
\hline \multirow[t]{2}{*}{ Pipe } & Entrance diameter $[\mathrm{mm}]$ & 25.9 \\
\hline & Material & Acrylic \\
\hline \multirow{6}{*}{$\begin{array}{l}\text { Flow } \\
\text { properties }\end{array}$} & Fluid & $10 \mathrm{~W}$ \\
\hline & Density $\left[\mathrm{Kg} / \mathrm{m}^{3}\right]$ & 1018 \\
\hline & Yield stress $[\mathrm{Pa}]$ & 0.07 \\
\hline & Consistency coefficient $\left[\mathrm{Pa} \cdot \mathrm{s}^{n}\right]$ & 0.002 \\
\hline & Flow behavior index [-] & 0.99 \\
\hline & $R e_{D}^{\prime}$ & $4000 ; 5000 ; 10000$ \\
\hline \multirow{3}{*}{$\begin{array}{l}\text { Particles } \\
\text { properties }\end{array}$} & Type & Spherical PSP-Rhodamine B \\
\hline & Density $\left[\mathrm{Kg} / \mathrm{m}^{3}\right]$ & 1150 \\
\hline & Average diameter $[\mu \mathrm{m}]$ & $5-10$ \\
\hline \multirow{4}{*}{$\begin{array}{l}\text { Laser } \\
\text { settings }\end{array}$} & Type & Double- Nd:YG \\
\hline & Wavelength [nm] & 532 \\
\hline & Energy $[\mathrm{mJ}]$ & 60 \\
\hline & Laser beam thick $[\mathrm{mm}]$ & 1 \\
\hline \multirow{3}{*}{$\begin{array}{l}\text { Camera } \\
\text { settings }\end{array}$} & Type & CMOS \\
\hline & Resolution [Pixels] & $2352 \times 1728$ \\
\hline & Trigger rate $[\mathrm{Hz}]$ & 50 \\
\hline \multirow[t]{4}{*}{ Image } & f-number & 8 \\
\hline & Visualization area $[\mathrm{mm} \times \mathrm{mm}]$ & $52 \times 26$ \\
\hline & Time between pulses $[\mu \mathrm{s}]$ & $230 ; 190 ; 95$ \\
\hline & Number of images & 1040 \\
\hline \multirow[t]{5}{*}{ PIV analysis } & Type of correlation & Central difference \\
\hline & Validation & Moving average \\
\hline & Interrogation area & $32 \times 32$ \\
\hline & Overlap & $50 \%$ \\
\hline & Vectors per image & $146 \times 107=15622$ \\
\hline
\end{tabular}

Source: Author (2019).

velocity distribution for a turbulent regime. At the entrance of the test section, the $u / \bar{U}$ reach values between 1.2 and 1.5 at the region $-0.4<r / D<0.4$, where the evidence of a flat distribution is distinguishable. Besides, the asymmetry behavior continues for the turbulent regime, as the $R e_{D}^{\prime}$ increases this behavior is more visible by the velocity scalar map distribution. As it was exposed for the analysis of the laminar regime, this asymmetry behavior also has been observed by Escudier and Presti (1996) and Peixinho et al. (2005), and it can be related with an increase of the inertial forces, which have a destabilizing effect and is responsible for a gradient sharpening, Nieuwstadt et al. (2016).

For Newtonian flow, values of $u / \bar{U}=3.55,3.48,3.46,3.45$, and 3.35 were obtained by (PALACIOS, 2011 ) for turbulent $R e_{D}=5303,10038,17758,24590$, and 40065 , respectively. Similar to the laminar case, a decrease of $u / \bar{U}$ and $v / \bar{U}$ was observed. However, these values are higher than the values found in the present study, which leads to state that the increase of $\tau_{0}^{H}$ reduces the $u / \bar{U}$ values due to a higher consistency coefficient of the viscoplastic material. 
Figure 5.11 - Mean axial velocity map distribution for turbulent $\left.R e_{D}^{\prime} \cdot \mathrm{a}\right), \mathrm{b}$ ), and c) show the maps for 10W fluid.
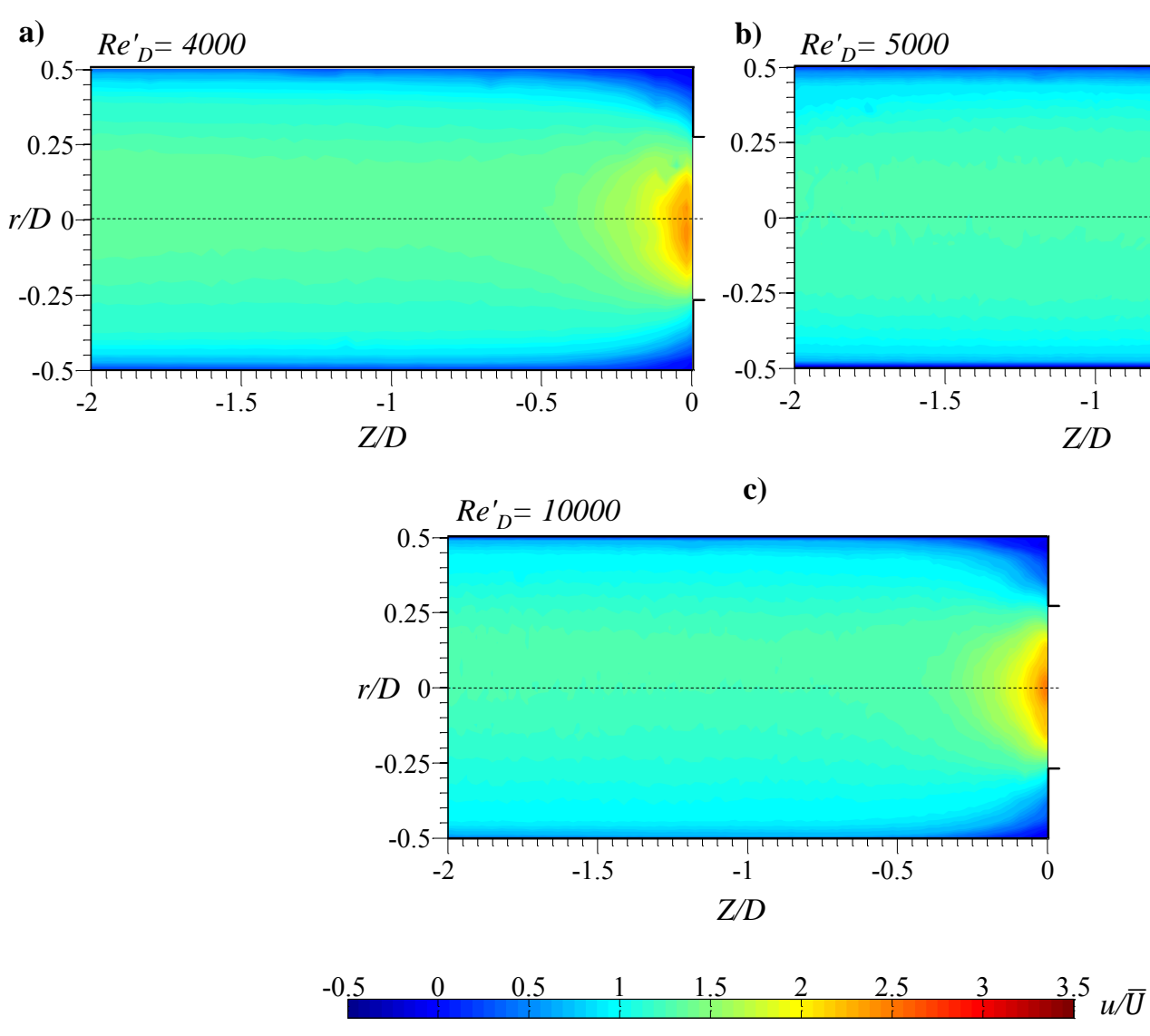

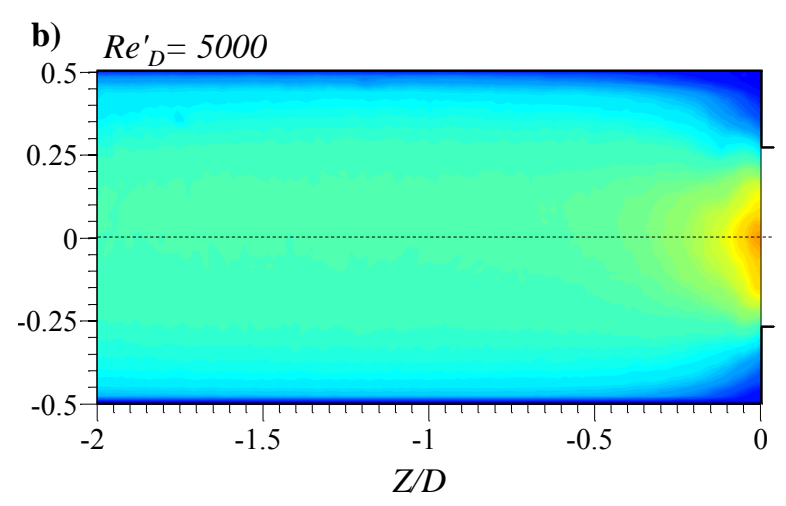

c)

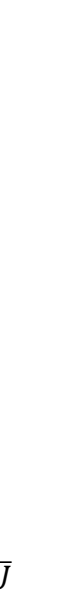

Source: Author (2019).

The radial dimensionless velocity maps present a similar flow structure of the laminar regime. Nevertheless, it is visible a decreasing of the maximum $v / \bar{U}$ values at the contraction plane, as compared with laminar $R e_{D}^{\prime}$. Also, an increase of the $R e_{D}^{\prime}$ leads to a reduction of the $v / \bar{U}$ ratios, which is the same correlation previously observed for the laminar regime. A maximum value of 1 is reached by a $R e_{D}^{\prime}=4000$, instead of 5000 and 10000 , the values are 0.86 and 0.81 respectively. It should also be noted that the area under the effect of the maximum values $v / \bar{U}$ seems to be constants after the turbulent regime is achieved.

For Newtonian fluids Palacios (2011) observed higher values $v / \bar{U}$ at the contraction plane than the present study. Values between 2.39 and 2.55 were obtained for Reynolds number between 5000 and 40000, which are higher than the laminar ones obtained in the same experimental study.

\subsubsection{Velocity Profiles}

The contractions effects are easily evaluated and measured comparing the axial and radial velocity profiles at different positions of the test section, also the analytical solution is obtained for each solution in order to compare the effects of the contraction. The velocity profiles were obtained for different $R e_{D}^{\prime}$ imposed for each viscoplastic solution and compared with Newtonian fluid cases available in the literature. 
Figure 5.12 - Mean radial velocity map for turbulent $\operatorname{Re}_{D}^{\prime}$. a), b), and c) show the maps for $10 \mathrm{~W}$ fluid.
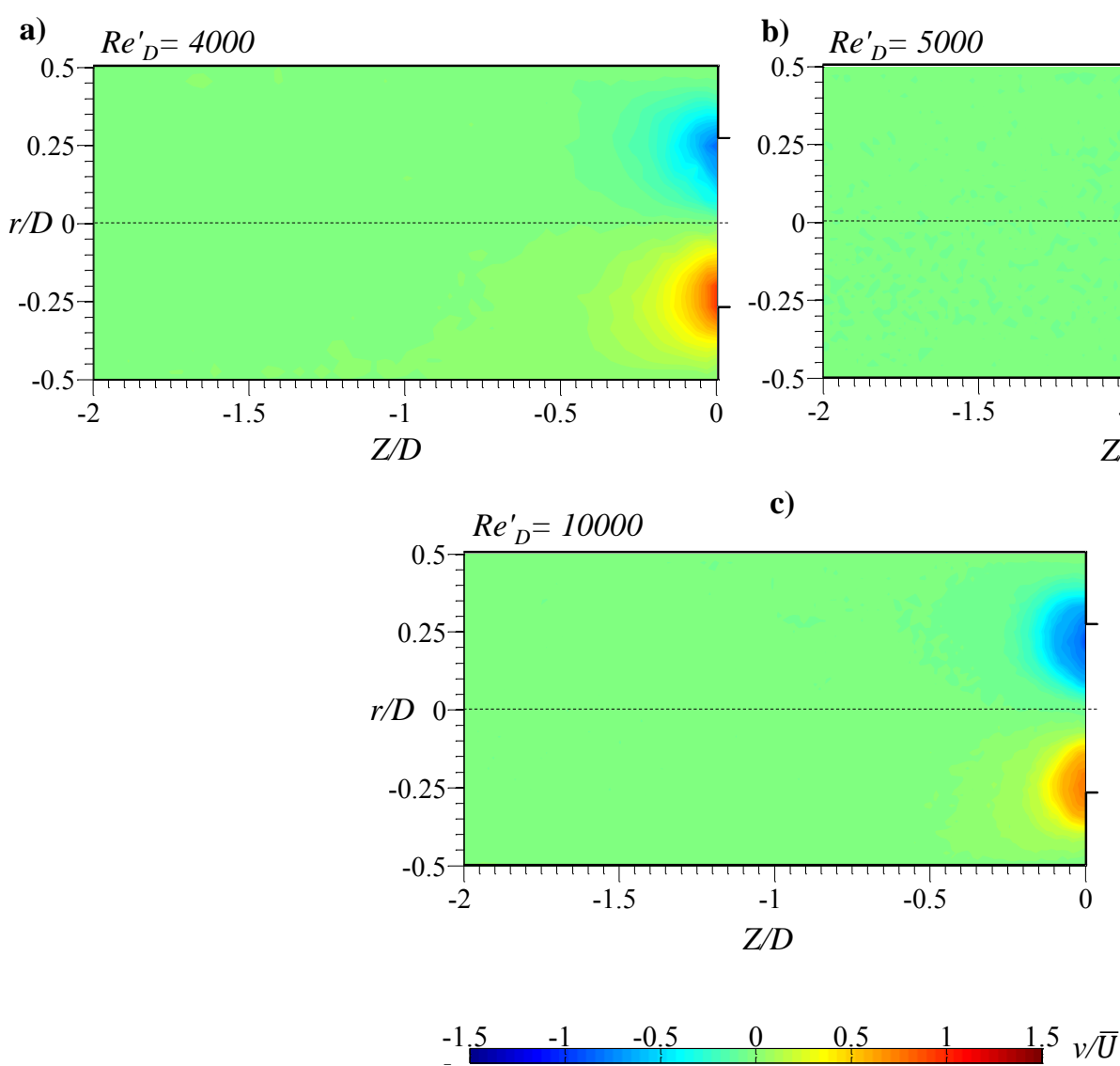

Source: Author (2019).

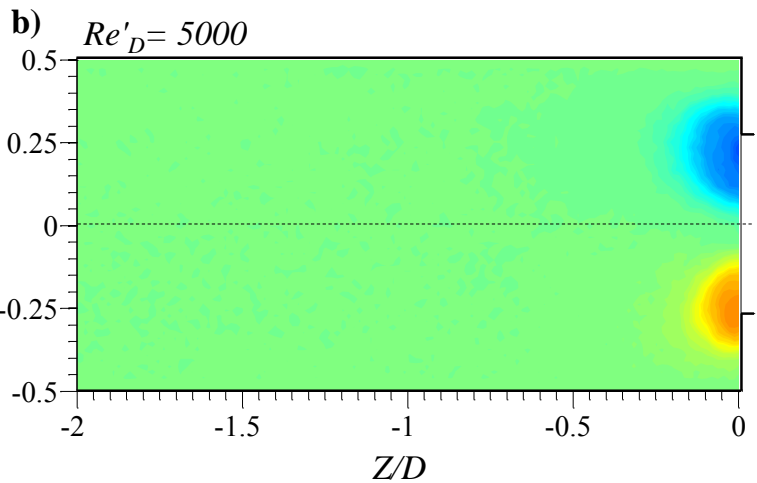

c)

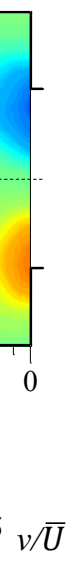
/ 
1500 , respectively.

The analytical solutions for each $R e_{D}^{\prime}$, and for each solution are obtained and compared with the profiles velocity at the entrance of the test section, when $Z / D=-2$. It was expected to obtain similar values, however there is a difference, thus the analytical solutions present maximal $u / \bar{U}$ values of 1.45 for $10 \mathrm{~W}$ solution, and 1.40 for $20 \mathrm{~W}$ solution. As noticed in Fig. 5.13, there is a maximum difference of $19.4 \%$ for both fluids. This difference can be explained by two conditions, the first is the slip at the wall of the pipe that is presented by polymers solutions as Carbopol ${ }^{\circledR}$ ones. The slip effect for viscoplastic material has been noticed in other experimental studies performed by Schmidt et al. (1999), Piau (2007) and Poumaere et al. (2014). The second one, it is the effect of the entrance to the test section with the contraction, seems that due to the acceleration of the fluid in this region the entrance profile presents higher values at the plug core region for laminar conditions. Nevertheless the difference between both profiles is due to directly effects of the special geometry used in this study.

Figure 5.13 - Mean axial velocity profiles for laminar regime: a) and b) for 10W; c), d), e) and f) for $20 \mathrm{~W}$. Red-dashed lines represent the central plug core size.
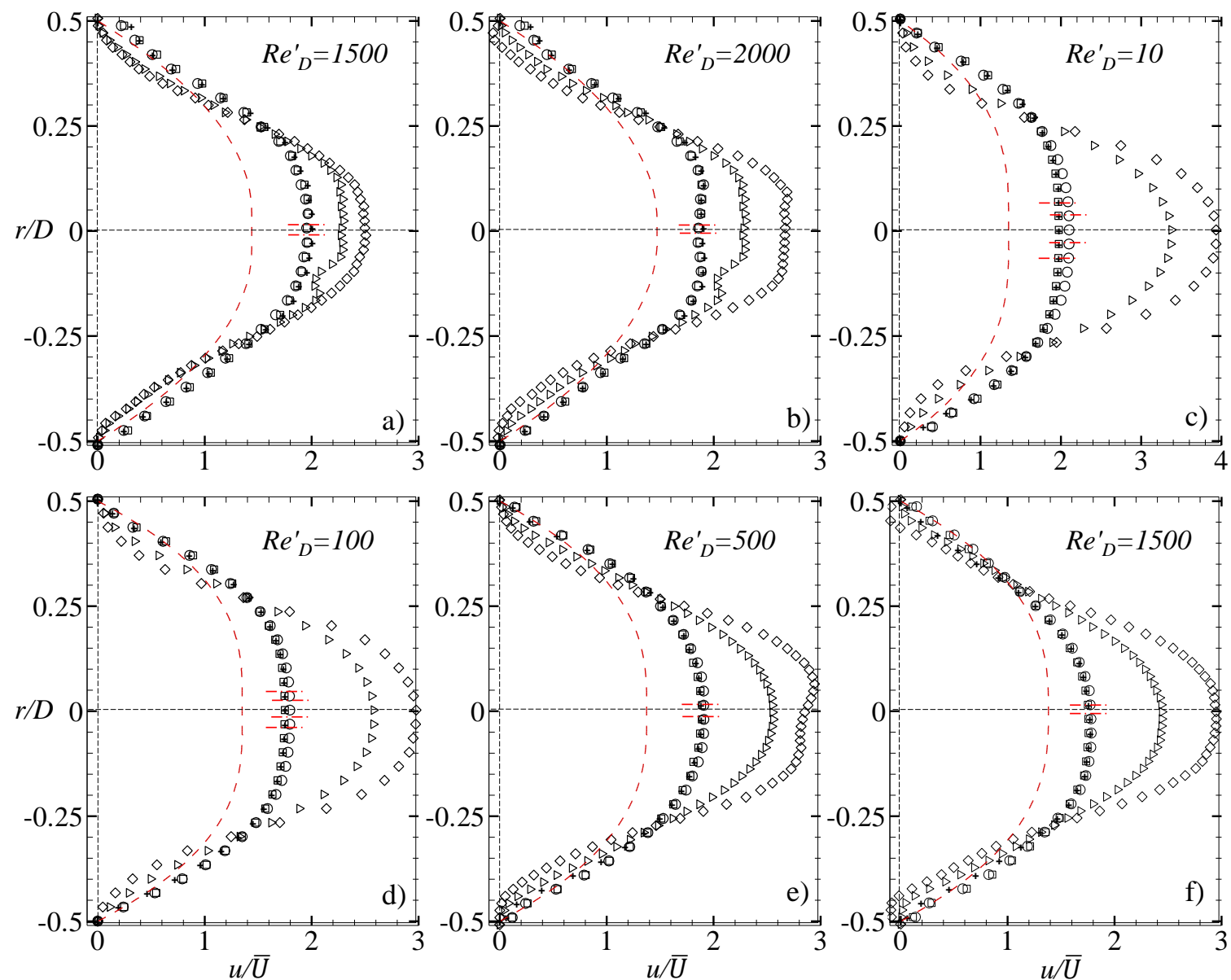

$+\mathrm{Z} / \mathrm{D}=-2$

$\mathrm{Z} / \mathrm{D}=-1$
$\mathrm{Z} / \mathrm{D}=-0.5$

$\triangleright \mathrm{Z} / \mathrm{D}=-0.1$

$\diamond \mathrm{Z} / \mathrm{D}=-0.02$

--- Analytical solution

Source: Author (2019). 
Figures 5.13a and 5.13b show instability of the profiles at the entrance of the contraction, an asymmetric behavior is observed as it was discussed for the axial velocity scalar maps. When the profiles reach $Z / D>-0.02$, reverse flows are observed due to the generation of vortex structures at the corner of the contraction, and the plug core structure is wholly yielded. The reverse flow is observed in Figs.5.13b and 5.13f.

For fluids $30 \mathrm{~W}$ and $40 \mathrm{~W}$, Fig. 5.14 shows the evolution of the axial velocity for different $R e_{D}^{\prime}$. Low $R e_{D}^{\prime}$ values were imposed for both fluids, at these conditions a greater plug core is visualized at $Z / D=-2$ than for fluids $10 \mathrm{~W}$ and $20 \mathrm{~W}$. The size of the plug core region is proportional to $\tau_{0}^{H}$ and $R e_{D}^{\prime}$ values, a typical behavior for viscoplastic fluid in pipe flows Hanks (1963), Hanks et al. (1967), Malin (1998), Peixinho et al. (2005).

The plug core for fluid 30W presents a radius of $0.2 D$ for $R e_{D}^{\prime}=10$ at $Z / D=-2$. As the $R e_{D}^{\prime}$ increases the radius is reduced to $0.1 D$ and $0.075 D$ for $R e_{D}^{\prime}$ of 100 and 500 . Instead for fluid $40 \mathrm{~W}$ the radius of the plug core is $0.275 D, 0.23 D$, and $0.13 D$ for $R e_{D}^{\prime}$ of 1,10 , and 100 , respectively.

Figure 5.14 - Mean axial velocity profiles in laminar regime: a), b), and c) shows the profiles for 30W; d), e), and f) for 40W. Red-dashed lines represent the central plug core size.
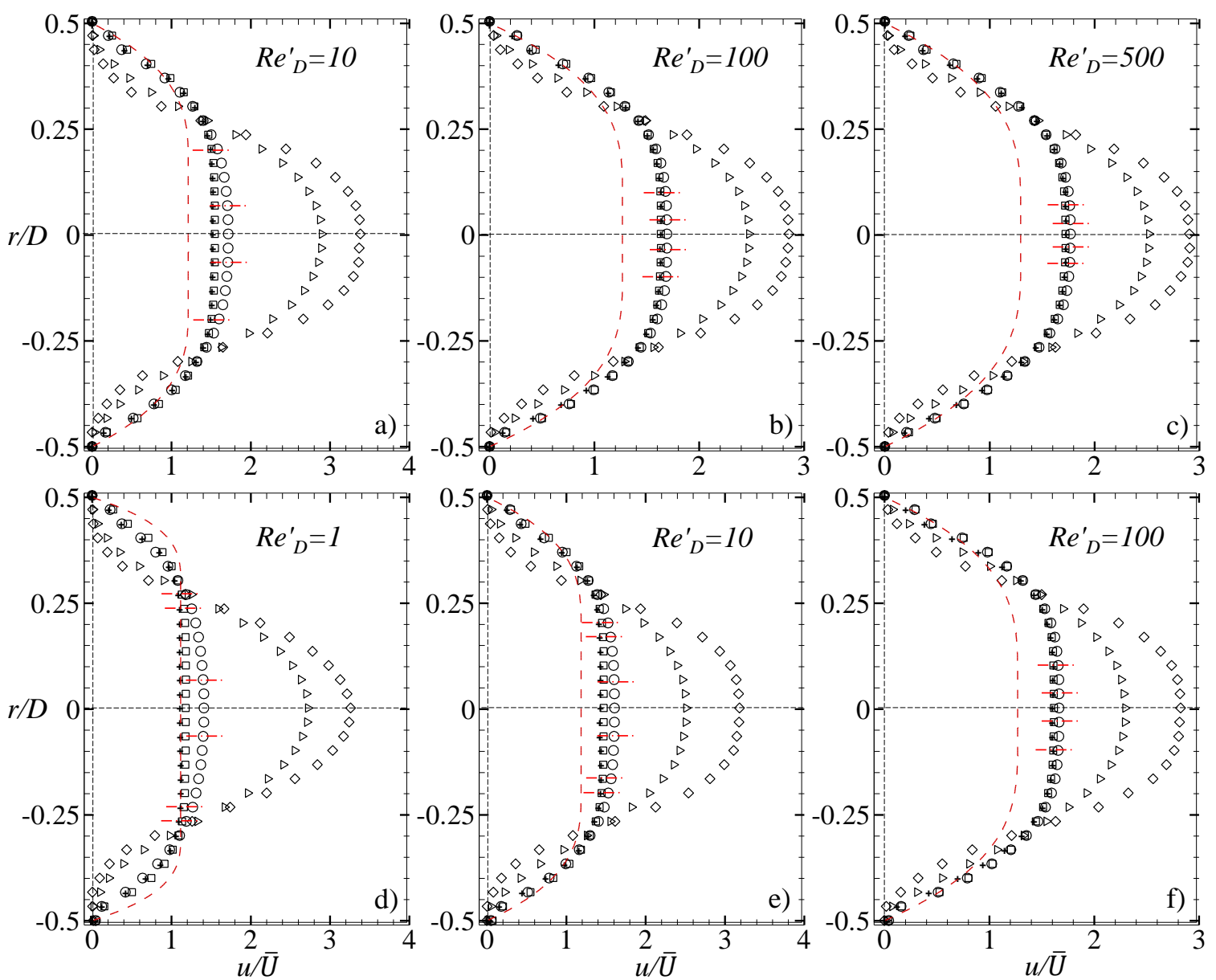

$+\mathrm{Z} / \mathrm{D}=-2$

$\mathrm{Z} / \mathrm{D}=-1$

$\triangleright \mathrm{Z} / \mathrm{D}=-0.1$

$\circ \mathrm{Z} / \mathrm{D}=-0.5$

$\diamond \mathrm{Z} / \mathrm{D}=-0.02$

-- Analytical solution

Source: Author (2019). 
The plug core region is reduced as the flow approaches the contraction plane, as presented in Fig. 5.14, and both fluids the radius of the core is significantly reduced for $Z / D=-0.1$. However, it is important to note that for low $R e_{D}^{\prime}$ and high $\tau_{0}^{H}$ the effects of the contraction appears at $Z / D \approx-0.5$, it is possible to state that as higher is the yield stress value, the fluid is more sensitive for effects of the contractions at low Reynolds numbers. Nevertheless, these effects are compared in the next subsections, comparing the centerline velocity for each viscoplastic fluids.

For fluids $30 \mathrm{~W}$ and $40 \mathrm{~W}$, the reverse flow is not visualized due to $R e_{D}^{\prime}$ did not achieve a critical value near to the transition regime, all the $R e_{D}^{\prime}$ performed are in the laminar regime. The $u / \bar{U}$ values for solution $30 \mathrm{~W}$ at the entrance of the section $(Z / D)$ is 1.55 for the $R e_{D}^{\prime}$ presented in Fig. 5.14. Then, the profile presents maximum $u / \bar{U}$ values near the contraction of 2.95 . Nevertheless, $R e_{D}^{\prime}=10$ presents the higher value at $Z / D=-0.02$ for both fluids. This behavior is explained in the next section due to it is related with the appearance of unyielded regions in the corner of the contraction.

The analytical solutions for laminar conditions is presented in Fig. 5.14. The maximum difference between the analytical solution and the profile at $Z / D=-2$ is $18.75 \%$ and $18.11 \%$ for $30 \mathrm{~W}$ and $40 \mathrm{~W}$, respectively. This differences are reach for the highest $R e_{D}^{\prime}$ imposed for each fluid. As explained before for fluids $10 \mathrm{~W}$ and $20 \mathrm{~W}$, this is behavior is generated by the presence of slip at the wall of the pipe and the acceleration of the fluid. As higher is the $R e_{D}^{\prime}$, higher will be the difference between the analytical and the experimental profiles obtained for each fluid. However, the difference is less than $20 \%$ and it is well represented the behavior of the viscoplastic fluids.

For the radial dimensionless velocity, a similar behavior for the axial velocity profiles is presented. At the same position along the test section for $10 \mathrm{~W}$ and $20 \mathrm{~W}$, it is noted that near to the transitional regime, the $v / \bar{U}$ ratio increases as compared with intermediate $\operatorname{Re}_{D}^{\prime}$ values. For low laminar $R e_{D}^{\prime}$ higher $v / \bar{U}$ are presented, and these values decrease until $R e_{D}^{\prime}$ near to the transitional regime are achieved. For example, for fluid 20W at $Z / D=-0.02$, a maximum $v / \bar{U}$ ratio of 1.1 for $R e_{D}^{\prime}=10$ is depicted by Fig. 5.15c. Whereas, $v / \bar{U}$ maximum values of 0.8 are presented for $R e_{D}^{\prime}=100$ and 500 . Then, the maximum ratio observed for $R e_{D}^{\prime}=1500$ is 0.85 , which is an increase of $6 \%$. Nevertheless, it is a lower value for $v / \bar{U}$, if it is compared with the ratio obtained for $R e_{D}^{\prime}=10$.

The radial dimensionless velocity profiles for fluids $30 \mathrm{~W}$ and $40 \mathrm{~W}$ are presented in Fig. 5.16, the same relation between the maximum $v / \bar{U}$ ratio and the $R e_{D}^{\prime}$ of fluid $20 \mathrm{~W}$ is observed. For low laminar $R e_{D}^{\prime}$, the maximum $v / \bar{U}$ is obtained, and this value decreases as the $R e_{D}^{\prime}$ increases. Moreover, for $40 \mathrm{~W}$ a particular behavior is shown in Fig. 5.16d, which depicts a maximum value for $v / \bar{U}$ ratio of 0.65 for $R e_{D}^{\prime}=10$. In conjunction with the axial dimensionless velocity profiles data obtained for $40 \mathrm{~W}$, it is possible to state that for low laminar $R e_{D}^{\prime}$, the large size of the plug core region and the low velocity gradients at the entrance of the contraction leads to low $v / \bar{U}$ values, due to a more uniform velocity distribution presented along the test section.

Figures 5.15 and 5.16 also show that the effects of the contractions are more distinguishable for the fluids with higher $\tau_{0}^{H}$ values at very low $R e_{D}^{\prime}$ when the flow reaches $Z / D=-0.5$. This behavior can be explained by the unyielded areas at the corner of the contractions that reduce the area and the angle at the entrance of the contraction. The reduction of the entrance angle pushes the plug core region back, promoting the flow of deformed material to the entrance of the contraction. Such effect is described 
Figure 5.15 - Dimensionless radial velocity profiles for laminar regime: a) and b) for 10W; c), d), e) and f) for $20 \mathrm{~W}$.
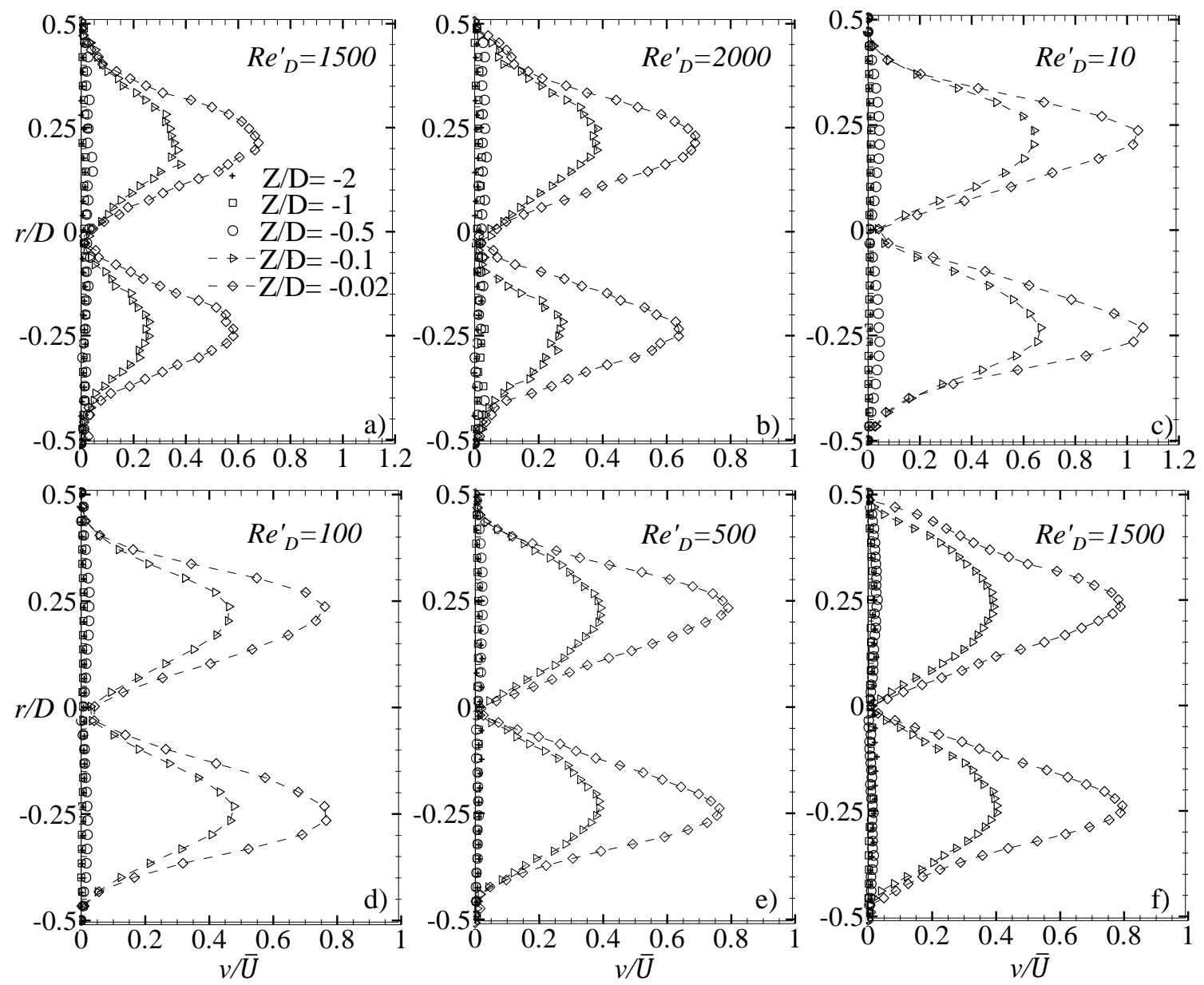

Source: Author (2019).

by numerical studies carried out by Gans (1999) and Jay et al. (2002). After the $R e_{D}^{\prime}$ is increased the unyielded regions at the corner of the contractions are reduced, and the effects due to the area reduction are observed at least for $Z / D=-0.1$. This effect is explained in detail in the following subsections of this chapter.

Peak values obtained for the radial velocity near the entrance of the contraction are generated by the impact of the flow with the walls and contraction corners. In this region the fluid is forced to go through the smaller diameter $(d)$, altering the radial component of the velocity.

The data obtained in this study for axial and radial velocity profiles are compared with data available in the literature for Newtonian fluids flow through abrupt contractions. A comparison between three laminar $R e_{D}^{\prime}$ is made using experimental data obtained from Durst and Loy (1985) using the LDA technique and a contraction ratio $\beta=1.87$, and the data obtained with the PIV technique by Palacios (2011) for a contraction ratio $\beta=1.97$.

Figure 5.17 depicts that for the different positions at the test section the Newtonian fluids present higher $u / \bar{U}$ values than the viscoplastic fluids. At the entrance of the test section similar values for $u / \bar{U}$ are observed for Newtonian and viscoplastic fluids, as shown in Fig. 5.17a for a $Z / D=-1.047$. Moreover, 
Figure 5.16 - Dimensionless radial velocity profiles for laminar regime: a), b), and c) for 30W; d), e), and f) for $40 \mathrm{~W}$.
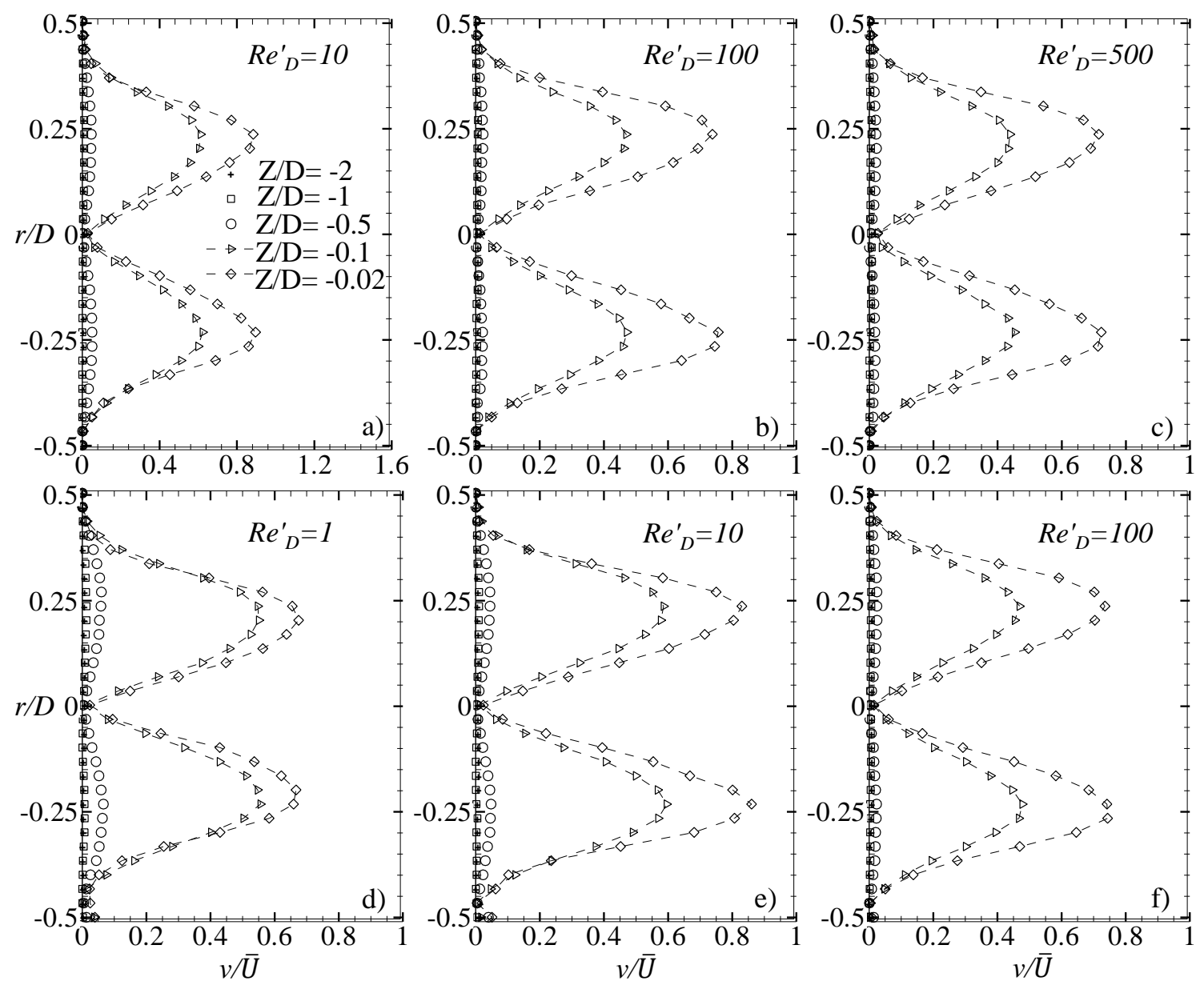

Source: Author (2019).

as the flow approaches to the contraction values for the axial dimensionless velocity for Newtonian fluids reaches values of $u / \bar{U}=3.8$, instead for viscoplastic fluid the ratio presents values of $u / \bar{U}=4$. Then, the Newtonian fluid flow is more sensible to the effects generated by the contraction. For the laminar regimes the Newtonian fluid displays a parabolic velocity profile, instead the viscoplastic fluid velocity profile has a flatted shape due to the existence of the yield stress. This behavior for viscoplastic fluids is compared for different experimental studies for straight pipes leaded by Schmidt et al. (1999), Peixinho et al. (2005), also in numerical studies fir area reductions effects performed by Duda and Vrentas (1973) and Sunarso et al. (2007).

For the radial profiles, the Fig. 5.18 shows the same behavior for the axial velocity profiles. The Newtonian flow presents higher values for $v / \bar{U}$, and these are increased as the flow approaches the contraction plane. It is possible to state that the presence of yield stress affects the flow structure of the fluid, reducing its sensibility to area reduction effects, since the viscous forces are increased. Also, this behavior can be stated as a function of the $H e_{H B}$, which relates the rheological properties for viscoplastic fluids.

The results presented in Fig. 5.18 suggest that as $R e_{D}^{\prime}$ increases, the radial velocity gradients increase 
Figure 5.17 - Comparison of the axial dimensionless velocity profiles between Newtonian and the viscoplastic fluids flow for the dimensionless axial position $Z / D=-1.047, Z / D=-0.104$, and -0.039 .
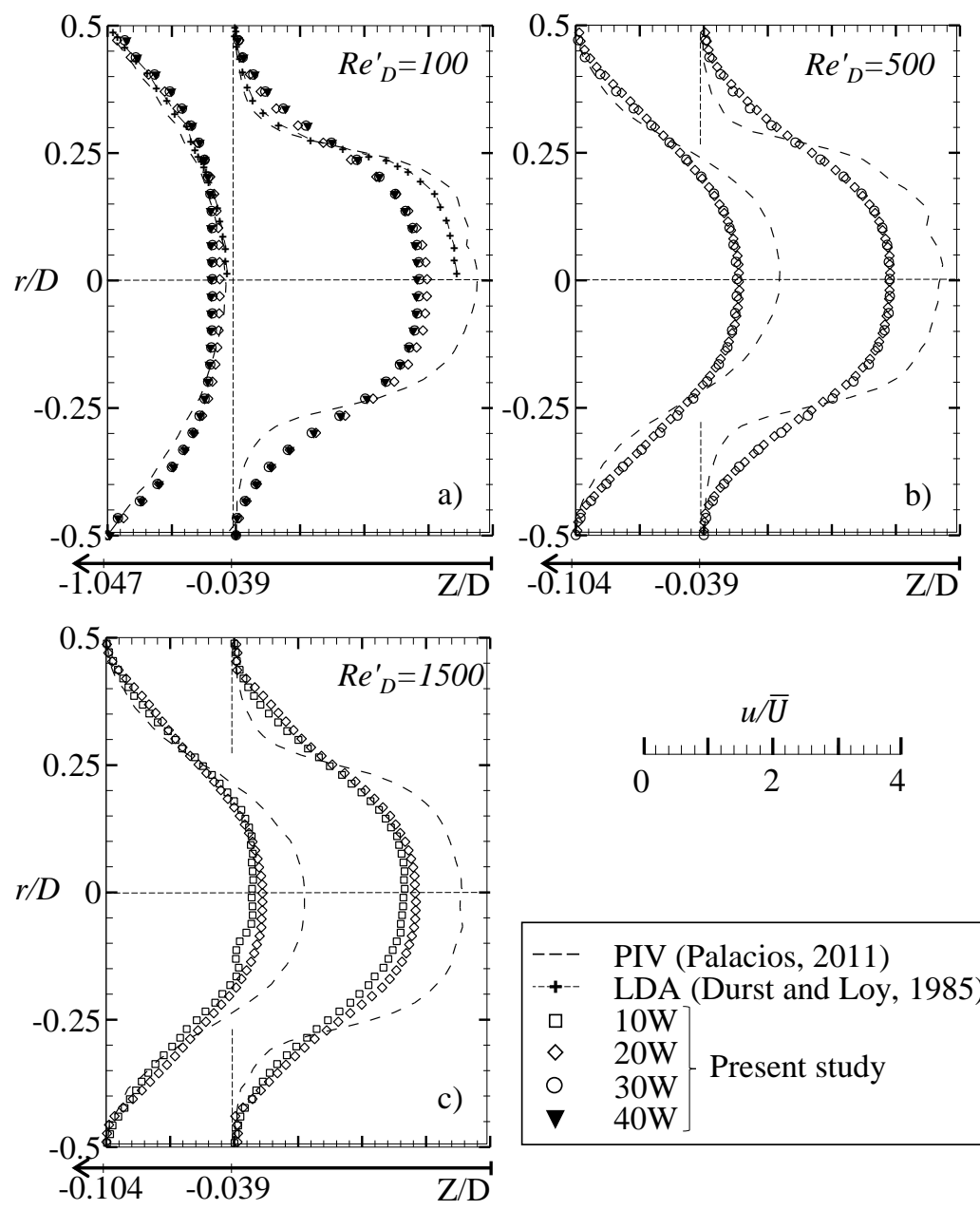

Source: Author (2019).

which is visualized with the increasing of the $v / \bar{U}$ ratio. It appears possible that the increased viscosity in the core region of the flow in viscoplastic fluids may result in lower fluctuations and gradients than in a Newtonian fluid as stated by Rudman et al. (2004).

As for Figure 5.18, which shows the radial component profiles of $v / \bar{U}$ analogously to Fig. 5.17, an asymmetric behavior is observed for $R e_{D}^{\prime}=1500$. Nevertheless, this behavior is not prominent for Newtonian fluids, and when the turbulent regime is reached the axial profile tends to a symmetry behavior again. Also, it is observed that for the viscoplastic fluids flow the $v / \bar{U}$ are similar near to the contraction entrance. However, when the contraction plane is achieved the maximum $u / \bar{U}$ and $v / \bar{U}$ values varies according to Figs. 5.7, 5.8, 5.9, and 5.10.

When compared with the data found in the literature, the behavior obtained for $u / \bar{U}$ and $v / \bar{U}$ ratios presents coherent trends and values. It is essential to highlight that the comparison with non-Newtonian fluids data is a challenge since there is no available data for the same rheological parameters. 
Figure 5.18 - Comparison of the radial dimensionless velocity profiles between Newtonian and the viscoplastic fluids flow for the dimensionless axial position $Z / D=-0.104$ and -0.039 .
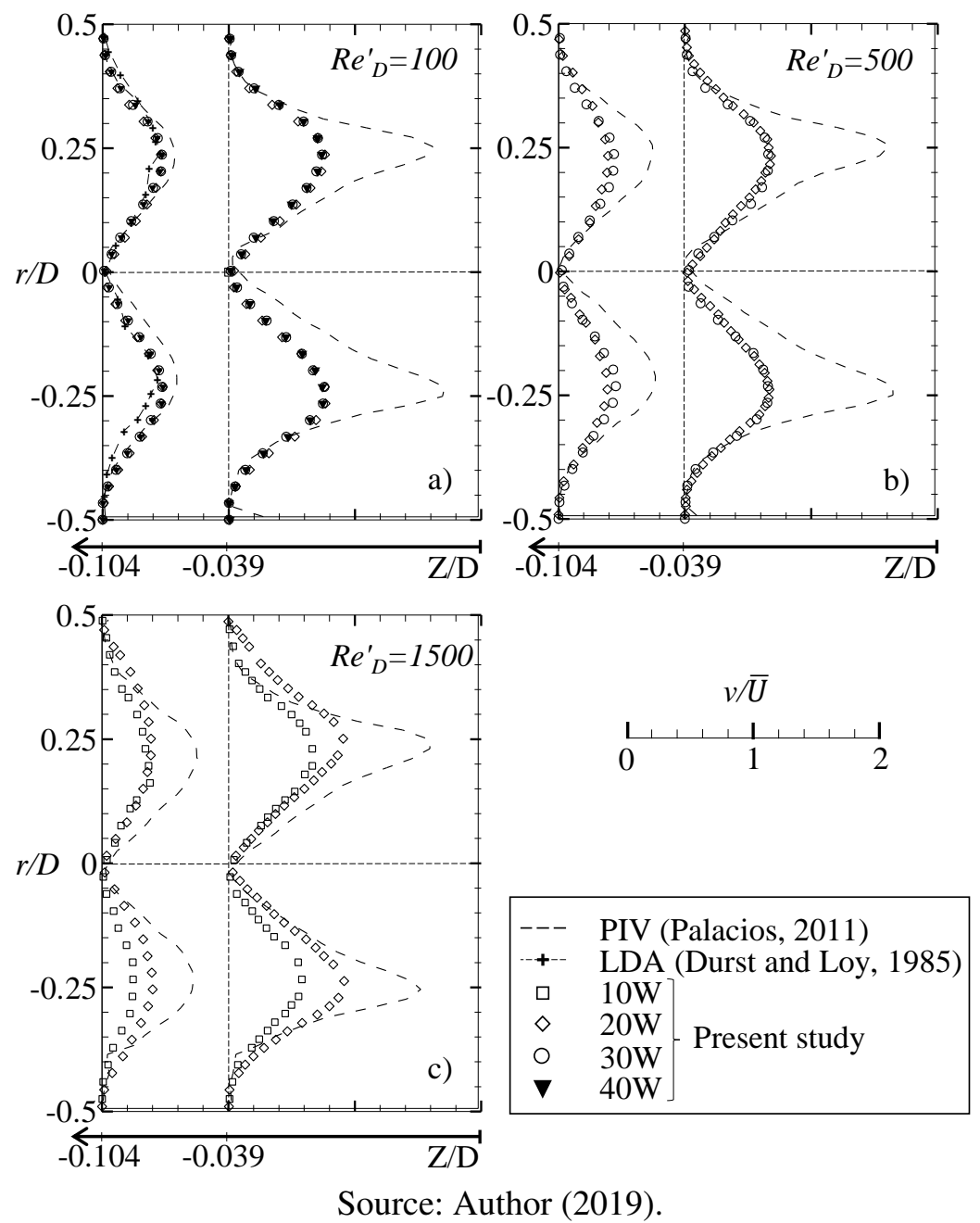

\subsubsection{Turbulent flow}

The results for the turbulent flow regime are presented in Fig. 5.19 for axial and radial dimensionless velocity profiles. For the turbulent regime just the data obtained for fluid $10 \mathrm{~W}$ are presented. The data are also compared with the experimental data obtained by Palacios (2011) for Newtonian fluid flow and $R e_{D}^{\prime}=5000$, and 10000 in an abrupt contraction of $\beta=1.97$.

Figure 5.19a presents the axial dimensionless velocity profiles, which display a flat profile at the entrance of the test section, similar to the profile described by a Newtonian fluid. After the flow approaches the contraction plane, the $u / \bar{U}$ ratio increases reaching values of 2.5 . The radial dimensionless velocity displays values of $v / \bar{U} \approx 0.04$ at the entrance of the test section, these values increase at the contraction plane with $v / \bar{U} \approx 0.7$. Nevertheless, the values for $u / \bar{U}$ and $v / \bar{U}$ are lower than the ratios observed for the laminar regime. It seems that for the turbulent regime, the ratio for the radial and axial velocity are more uniform at the test section since the flow structure is completely yielded.

The axial dimensionless velocity shows a sharp profile at the entrance of the contraction; it appears to be caused by the high inertial forces present in the turbulent regime, that have a destabilizing effect. On 
Figure 5.19 - Mean velocity profiles for fluid $10 \mathrm{~W}\left(H e_{H B} \approx 0\right)$ at turbulent regime: $\left.\left.\mathrm{a}\right), \mathrm{b}\right)$, and c) display the axial dimensionless velocity; d), e), and f) the radial velocity ratio.
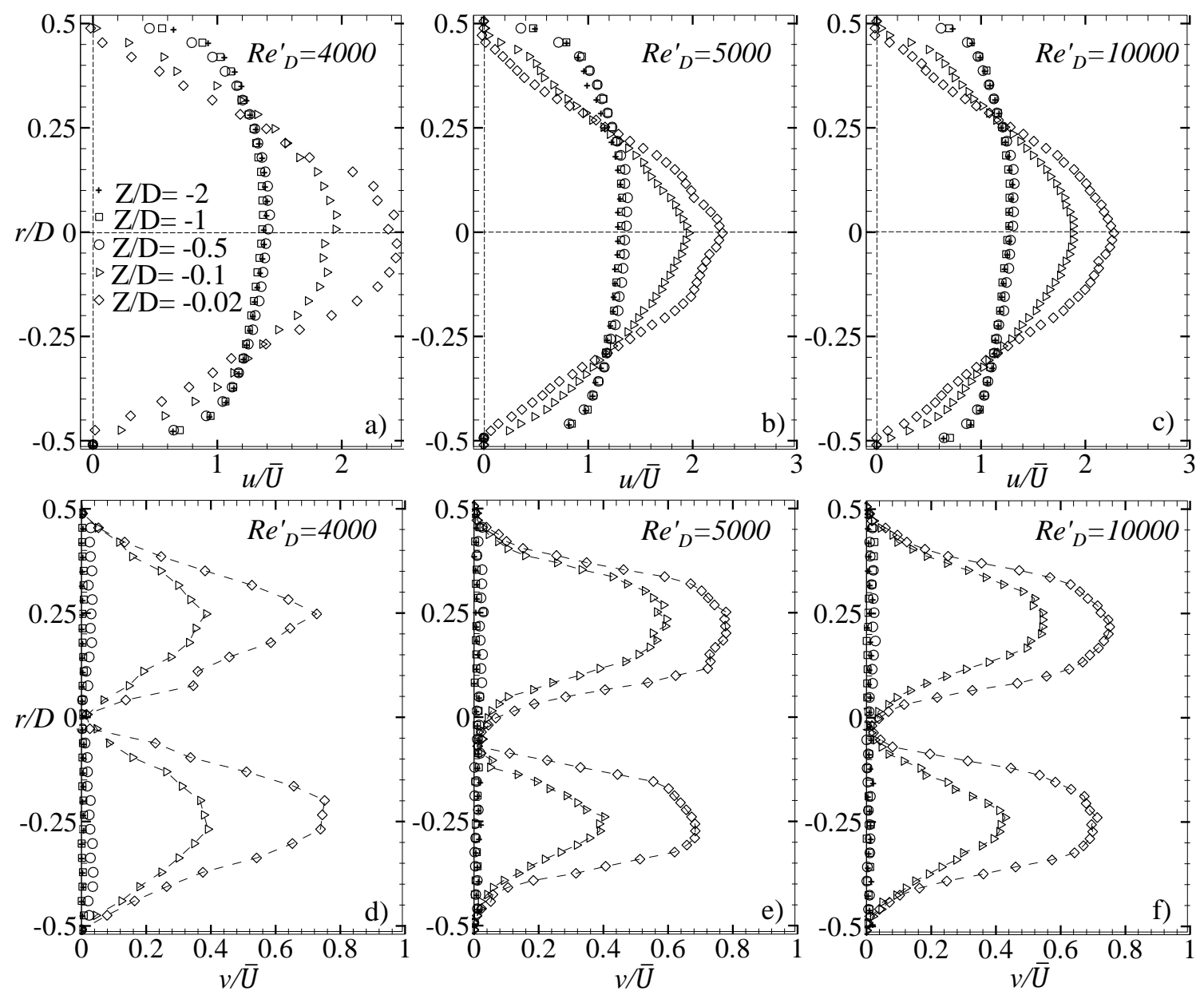

Source: Author (2019).

the other hand, the radial dimensionless velocity presents a rounded profile near the contraction. When compared with the data obtained by Palacios (2011), it is noted that the viscoplastic flow shows lower $u / \bar{U}$ values for the different positions depicted in Figs. 5.20a and 5.20b. At the entrance of the test section, the $u / \bar{U}$ values for the Newtonian and viscoplastic fluids are 2.6 and 1.8, respectively. Nevertheless, it is noted that the values for the Newtonian fluids are slightly higher than the viscoplastic axial dimensionless profile; this behavior is shown by Rudman et al. (2004). As well as for the laminar regime, the Newtonian fluid flow is more sensitive to the effects of the contraction, due to higher values for $u / \bar{U}$ are displayed by the Newtonian fluid when approaches to the contraction plane, where values of 3.6 are reached by the Newtonian fluids and 2.4 for the viscoplastic fluid.

The radial dimensionless velocity for viscoplastic fluids also presents lower values than the Newtonian fluids for each position along the test section. This comparison suggests that Newtonian fluids present higher gradients of velocity near the area reduction.

The shape of the profiles depicts different behavior for both fluids. The Newtonian fluid presents peaks of maximum values for $v / \bar{U}$ at the $r / D$ positions of the contraction, values near to 2.6. Instead, for viscoplastic fluids flow the $v / \bar{U}$ ratios depict a rounded shape near to the entrance of the contraction, 
Figure 5.20 - Comparison of the axial and radial velocity profiles for fluid 10W with Newtonian fluids for turbulent regime: a) and c) show the axial profile ratios for $R e_{D}^{\prime}=5000$; b) and d) display the radial velocity ratio for $R e_{D}^{\prime}=10000$.
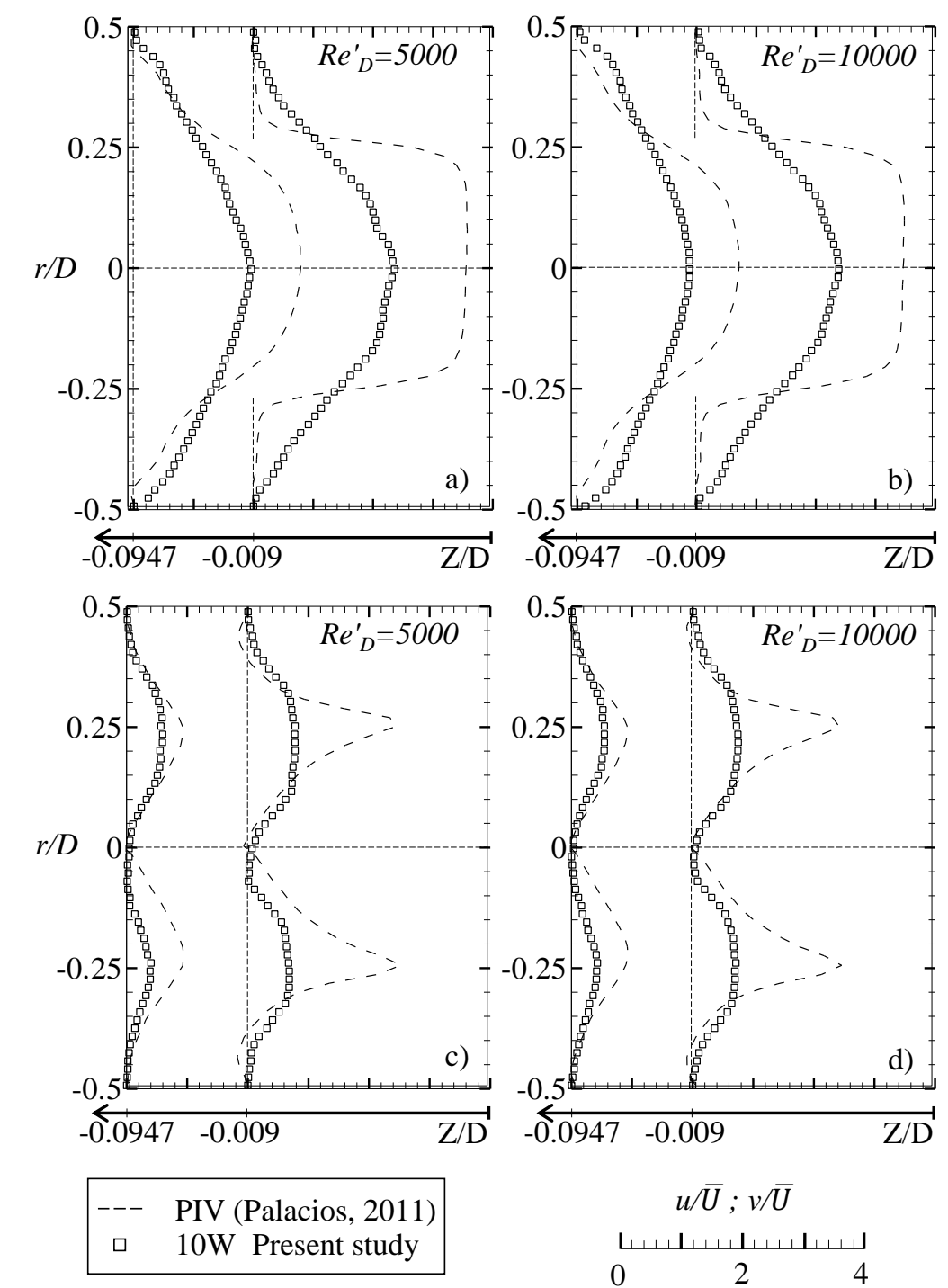

Source: Author (2019).

reaching values near to 0.8 . The sensibility of the viscoplastic fluids is explained in the next subsection through the analysis of the centerline velocities.

\subsubsection{Centerline Velocity}

In order to observe and compare the trends between the different velocity fields measured with the PIV technique and the variation for the different fluids studied and the different $R e_{D}^{\prime}$, this subsection analyzes the behavior of the centerline velocity $\left(u_{C L}\right)$. The Figure 5.21 presents the velocity over the centerline, $u_{C L}$, nondimensionalized by $\bar{U}$, as a function of the axial position $Z / D$. As exposed in the section 3.2 and subsection 5.2.1, the maximum values for $u / \bar{U}$ are obtained at the centerline $(r / D=0)$. These values increase as the flow approaches to the contraction plane $(Z / D=0)$. 
The higher values for $u_{C L} / \bar{U}$ are presented for the laminar regime along with the $Z / D$ position for each viscoplastic fluid. The $u_{C L} / \bar{U}$ values are between 1.5 and 2 at the entrance of the test section, for a position of $Z / D=-1.5$, and reaches values of 2.8 and 4 at the contraction plane. Fluid $40 \mathrm{~W}$ presents a particular behavior for a $R e_{D}^{\prime}=1$, the value of $u_{C L} / \bar{U}$ is 1.1 at the entrance of the test section, and reaches values of 3.5 at the contraction plane. This is an unexpected behavior that can be related to the low shear rates generated for this $R e_{D}^{\prime}$, and the high $\tau_{0}^{H}$ value. The plug core area is at least $30 \%$ of the test section entrance area, and the velocity at the centerline is accelerated due to the presence of large unyielded regions at the contractions.

For $R e_{D}^{\prime}$ in the laminar regime is depicted that as the $\tau_{0}^{H}$ increased the value for $u_{C L} / \bar{U}$ ratio at the contraction plane decreases. Due to for laminar Reynolds numbers, a value of $u_{C L} / \bar{U} \approx 4$ is reached by fluid $20 \mathrm{~W}$, and 3.5 for fluids $30 \mathrm{~W}$ and $40 \mathrm{~W}$.

For the turbulent regime, at the entrance of the test section are displayed values between 1.2 and 1.3, and begin to raise after $Z / D \approx-0.7$ is achieved. Analogously to the case of the laminar regime, the maximum values of $u_{C L} / \bar{U}$ are obtained in the section near to the plane of the contraction, being the values obtained in the order of $u_{C L} / \bar{U} \approx 2.5$.

Figure 5.21 - Axial dimensionless velocity along the centerline, $u_{C L} / \bar{U}$, as a function of the axial position $Z / D$, and the Reynolds number $R e_{D}^{\prime}$.

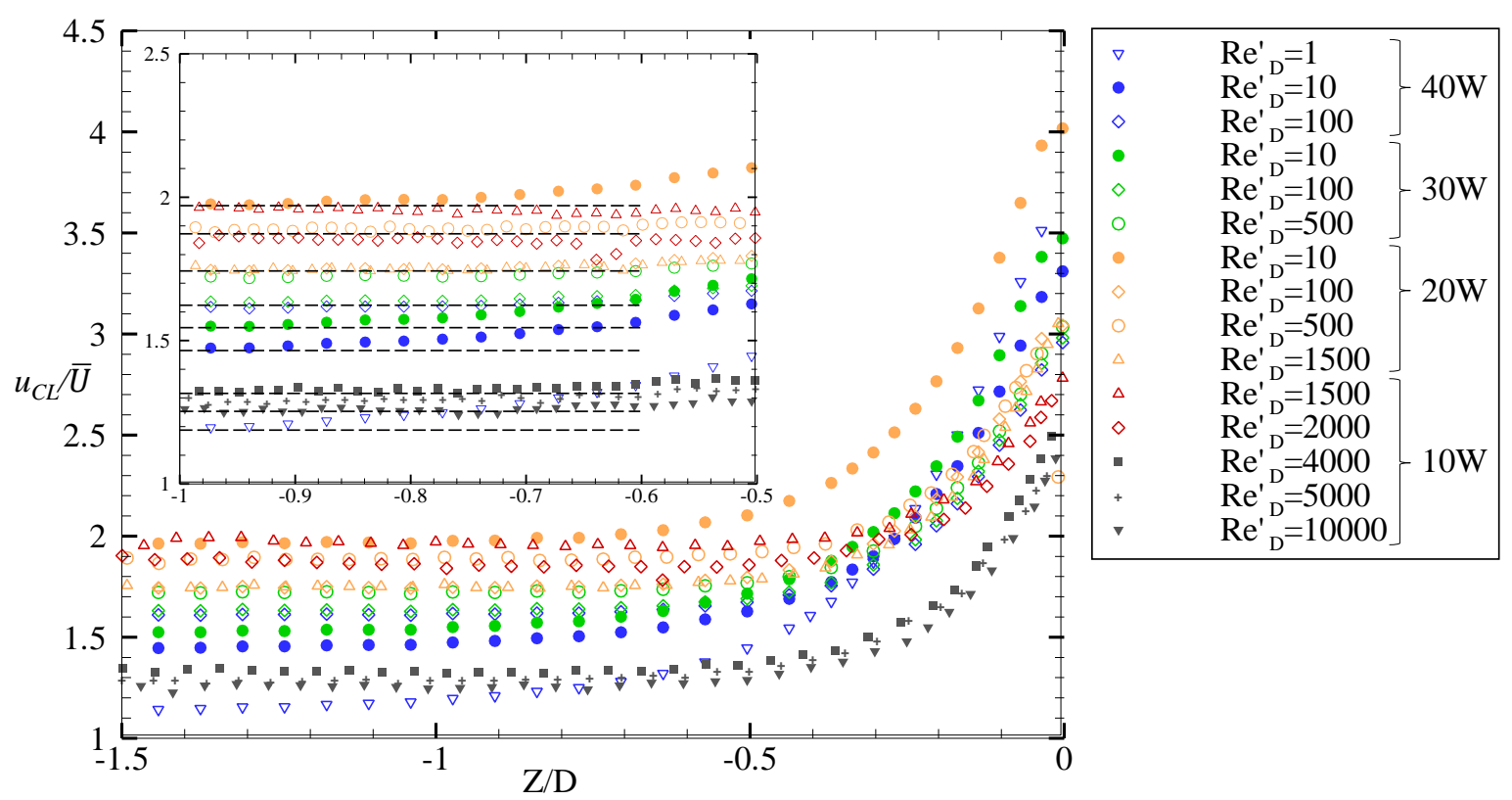

Source: Author (2019).

It is also observed in Fig. 5.21, that the values for $u_{C L} / \bar{U}$ in laminar regime, away from the contraction plane $(Z / D \leq-1)$ are apparently dependent of the rheological properties and the $\operatorname{Re}_{D}^{\prime}$, since for fluids $40 \mathrm{~W}$ and $30 \mathrm{~W}$ with a similar $H e_{H B}$, the $u_{C L} / \bar{U}$ values are lower than for fluids $10 \mathrm{~W}$ and $20 \mathrm{~W}$ with lower $H e_{H B}$. Also, the values among the $R e_{D}^{\prime}$ for each fluid have a slight variation, it is possible to state that the ratio $u_{C L} / \bar{U}$ it is not dependent of the $R e_{D}^{\prime}$, with the exception of very low $R e_{D}^{\prime}$ as exposed previously in this section, which presents a particular behavior. However, it is observed that for similar 
positions of $Z / D$ in the turbulent regime, fluid $10 \mathrm{~W}$ presents similar values of $u_{C L} / \bar{U}$ as evaluated for each turbulent $R e_{D}^{\prime}$.

The effects of the contraction on the viscoplastic flow are shown in Fig. 5.21 for axial positions between $-1 \leq Z / D \leq-0.5$. For the laminar and turbulent regime, it is displayed a horizontal trend of the $u_{C L} / \bar{U}$ until an axial dimensionless position of $Z / D=-0.6$ is reached, where a variation of the velocity is noticed. Nonetheless, as described before, for low $\operatorname{Re}_{D}^{\prime}$ values, the viscoplastic flows display variation in the dimensionless centerline velocity for $Z / D=-0.9$, due to there is a detachment of the experimental data from the dashed horizontal line depicted in Fig. 5.21. It is suggested that viscoplastic fluids are more sensitive to the effects of the contraction for low $R e_{D}^{\prime}$, apparently for the presence of unyielded regions at the entrance of the contraction that reduces the area of flow and increases the velocity gradients at the central position of the test section.

\subsubsection{Turbulent Statistics}

In this subsection, the turbulent quantities are evaluated for fluid $10 \mathrm{~W}$, and the different $R e_{D}^{\prime}$ studied.

For the calculation of the turbulent quantities, the average field of the velocities in the axial and radial directions, $u(r, z)$ and $v(r, z)$ respectively, are used, and the mean statistic turbulent fluctuations are calculated by the root mean square (rms) definitions (MAO; JAWORSKI, 2010), given by equations:

$$
\begin{aligned}
u^{\prime}(r, z) & =\sqrt{\frac{1}{N} \sum_{i=1}^{N}\left(u_{i}(r, z)-u(r, z)\right)^{2}} \\
v^{\prime}(r, z) & =\sqrt{\frac{1}{N} \sum_{i=1}^{N}\left(v_{i}(r, z)-v(r, z)\right)^{2}}
\end{aligned}
$$

where $u^{\prime}$ and $v^{\prime}$ are the fluctuations for velocities $u$ and $v$, along axial and radial directions, respectively; and $N$ is the number of vector fields obtained by the PIV technique.

Similar to the results presented for the mean velocities, the values obtained for the velocity fluctuations are nondimensionalized by the entrance velocity $\bar{U}$. The figure 5.22 presents the turbulent fluctuations for axial and radial velocity. It is possible to observe that fluctuations for the axial velocity at the entrance of the test section have symmetry behavior for each $R e_{D}^{\prime}$. After the flow reaches $Z / D=-0.5$, the fluctuations increase, and there are no more symmetry conditions. The high gradient velocity and the abrupt change of area increases the fluctuations at the position $Z / D=-0.5$, which are $u^{\prime} / \bar{U} \approx 0.3$ for $R e_{D}^{\prime}=4000$ and varies to $u^{\prime} / \bar{U} \approx 0.5$ and $u^{\prime} / \bar{U} \approx 0.4$ for $R e_{D}^{\prime}=5000$, and $R e_{D}^{\prime}=10000$, respectively. The $u^{\prime} / \bar{U}$ values for $R e_{D}^{\prime}=5000$ are higher than $R e_{D}^{\prime}=10000$ at $Z / D=-0.5$ due to the flow is entering to turbulent conditions, then after reach $R e_{D}^{\prime}=10000$ the fluctuations are increased at the side where is presented the asymmetry behavior for the flow depicted in Fig. 5.11. Far from the effects of the contractions, the peak values are displayed near to the pipe walls $(0.45 \leq|r / D| \leq 0.5)$, where Fig. 5.22a, b, and c depict values $u^{\prime} / \bar{U} \approx 0.2$.

For radial velocity fluctuations Fig. 5.22d, e, and f present defined profiles for each $R e_{D}^{\prime}$ at the entrance of the test section. Similar to the $u^{\prime} / \bar{U}$, the values for $v^{\prime} / \bar{U}$ increase as the flow approaches to 
Figure 5.22 - Turbulent intensities: axial and radial fluctuations for turbulent $R e_{D}^{\prime}$, plotted as a function of the dimensionless radial coordinate.
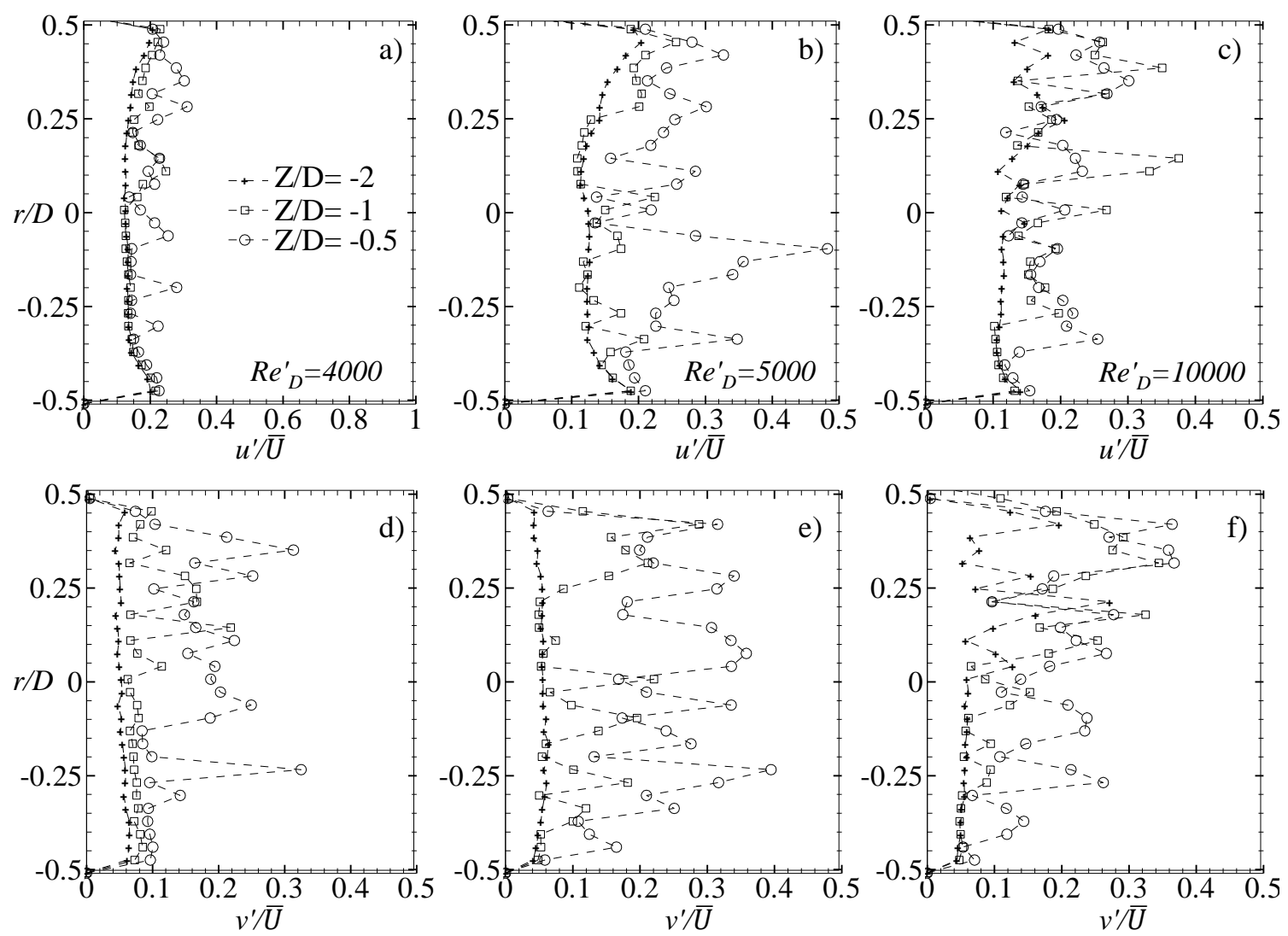

Source: Author (2019).

$Z / D=-0.5$, values are increased to 0.3 for $R e_{D}^{\prime}=4000$ and 10000 , and values of 0.4 for $R e_{D}^{\prime}=5000$. Also, the asymmetry behavior at the turbulent regime affects the radial fluctuations, leading to high fluctuations values for $r / D=0.5$, even for values near to the entrance of the test section for $R e_{D}^{\prime}=$ 10000.

The abrupt contraction configuration leads to particular behavior for the viscoplastic flow. The turbulent intensities are modified by the presence of the contraction, which increases the velocity gradients and consequently, the stability of the flow leading to high fluctuations values. At the entrance of the test section, far from the contraction, it is possible to obtain turbulent intensities profiles for a straight pipe configuration. Similar to the axial fluctuations, the maximum $v^{\prime} / \bar{U}$ values are obtained near to the pipe walls $(0.4 \leq|r / D| \leq 0.5)$, values of $v^{\prime} / \bar{U} \approx 0.05$.

The values obtained at the entrance of the test section can be compared with data available in the literature. Rudman et al. (2004) modelled non-Newtonian fluid flows using direct numerical simulation (DNS). The fluid used was a $0.05 \%$ wt Ultrez 10 solution, modelled as Herschel-Bulkley fluid, with $\tau_{0}^{H}=1.35, m=1.203$ and a flow index $n=0.52$.

Figure 5.23 depicts the comparison of the numerical data obtained by (RUDMAN et al., 2004) for $R e_{D}^{\prime}=5800$ and 8130 , and the experimental data obtained in the present study. The data are compared for $R e_{D}^{\prime}=5000$ and $R e_{D}^{\prime}=10000$, due to similar values for the turbulent regime. In order to compare 
Figure 5.23 - Turbulent intensities: axial and radial fluctuations for turbulent $R e_{D}^{\prime}$, plotted as a function of the dimensionless radial coordinate.
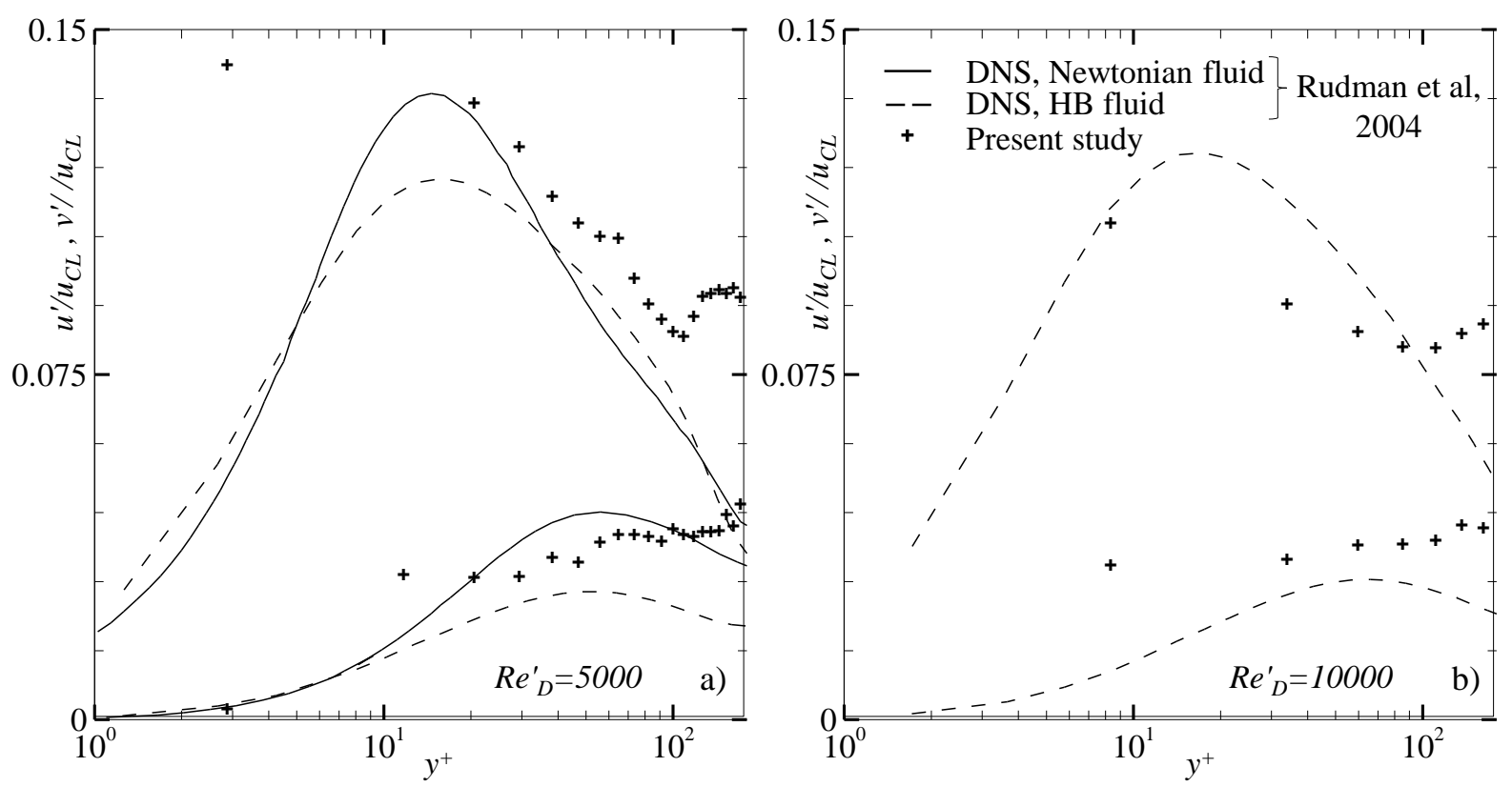

Source: Author (2019).

the experimental and DNS, the fluctuations are nondimensionalized by the centerline velocity $\left(u_{C L}\right)$, and the radial distance is presented in conventional wall units based on the mean wall viscosity, given by the following equations (RUDMAN et al., 2004):

$$
y^{+}=\frac{\rho u_{w}(r-R)}{\eta_{w}}
$$

where the friction velocity is defined as $u_{w}=\sqrt{\tau_{w} / \rho}$, and the wall viscosity is given by:

$$
\eta_{w}=m^{1 / n} \frac{\tau_{w}}{\left(\tau_{w}-\tau_{0}^{H}\right)^{1 / n}}
$$

Good representation of the axial and radial turbulent fluctuations is seen in Fig. 5.23, with a different of $18 \%$ for axial fluctuations, and $15 \%$ for the radial fluctuations. The values for the ratio $u^{\prime} / u_{C L}$ reach 0.15 , and $v^{\prime} / u_{C L}$ trends to 0.045 for both turbulent $R e_{D}^{\prime}$. It is possible to state that at turbulent conditions the Herschel-Bulkley fluids have not presented a dependence of the yield stress values, hence the rheological parameters for fluid 10W are different for the data used in DNS. This suggests a very little effect of the yield stress on the first order turbulence statistic. Also, it is possible to confirmed that at turbulent conditions, the log-law profile for the axial and radial fluctuations of the Herschel-Bulkley flows lay above the Newtonian profile with similar behavior. Nevertheless, the different between DNS and experimental data can be generated by the asymmetry condition developed in this flow regime.

It it necessary to highlight that experimental data obtained for $u^{\prime} / u_{C L}$ and $v^{\prime} / u_{C L}$ are influenced by external parameters to the experimental setup such as noise, source of error and the nature of the flow. Also, it is observed that $u^{\prime} / u_{C L}$ has more stable trends when compared to $v^{\prime} / u_{C L}$. This behavior is believed to be due to the measured order of magnitude, since as seen in Fig. 5.23 the $u^{\prime} / u_{C L}$ values 
are higher than those obtained for $v^{\prime} / u_{C L}$. Also, in Figs. 5.22 and 5.23, there is an uncertainty referred to the statistical convergence, such as the number of images used for the correlation of the turbulence profiles. However, this is a limitation of the camera storage capacity and it is necessary to recourse to other techniques to obtain a better convergence, such as LDV.

\subsection{VORTEX STRUCTURES AND UNYIELDED REGIONS}

The behavior of the flow structure at the corner of the contractions shows essential information to understand the Herschel-Bulkley flow behavior. Most experimental studies are focused on qualitative descriptions of the vortex generation. In this section is analyzed the behavior of the unyielded regions at the corners of the contraction, and the phenomena of generation and growth of the vortex structures. The analysis is carried out using quantitative methods and calculations.

\subsubsection{Visualization of the Contraction Corners}

The corners at the entrance of the contraction are visualized using the PIV technique. The statistic vector maps of the flow for each fluid at the different $R e_{D}^{\prime}$ are generated and allow to visualize the flow at this region. Figure 5.24 presents the evolution of the flow structure for fluids $10 \mathrm{~W}$ and $20 \mathrm{~W}$ for upper and lower corner as a function of the $\tau_{0}^{H}$ and $R e_{D}^{\prime}$.

It is important to note that for laminar regimes, the seeding particles tend to adhere to the wall, which is a common difficulty for regions with recirculation, in addition to those derived from the image acquisition, such as light refraction, the difference in the refractive indexes, and the pipe curvature. Moreover, for viscoplastic flows, the unyielded regions with a solid-like behavior retain the particles inside the solid structure, generating a region with spurious vectors. These regions at the corners with spurious vector are generated by a solid particles agglomerated in these regions due to zero velocity. Red dashed-lines delimit these spurious vector regions and allow to understand the behavior of the unyielded material among the different $R e_{D}^{\prime}$ set for each fluid.

Figure 5.24a displays the formation of the vortex structure at both corners for $10 \mathrm{~W}$ fluid with $\tau_{0}^{H}=0.07 \mathrm{~Pa}$, in laminar conditions. However, the lower corner does not show the same pattern of flow. As the $R e_{D}^{\prime}$ is increased to the turbulent regime, the vortex structures are completely formed, and growth until $R e_{D}^{\prime}=5000$ is reached. For a $R e_{D}^{\prime}=10000$, the Fig. 5.24e depicts small regions with recirculation at the corners. This reduction of the vortex structures shows that a significant increase of the flow leads to press the fraction of recirculating fluid against the wall, reducing its size.

On the other hand, 20W fluid with $\tau_{0}^{H}=0.26 \mathrm{~Pa}$ flow presents unyielded regions at the corner regions for laminar conditions. It is possible to see some spurious vectors at the corners for laminar $R e_{D}^{\prime}$, due to there is no movement detected for the seeding particles. The red dashed-line delimited the areas with the presence of statistic velocity vectors surrounded by spurious vectors. These areas delimit the unyielded regions, which are reduced as the $R e_{D}^{\prime}$ is increased. The non-deformed regions are reduced from a triangular area $A_{T r} \approx 1.5 \times 10^{-3} D^{2}$ for $R e_{D}^{\prime}=10$ to a $A_{T r} \approx 1.13 \times 10^{-3} D^{2}$, and $A_{T r} \approx 6.25 \times 10^{-4} D^{2}$ for $R e_{D}^{\prime}=100$ and $R e_{D}^{\prime}=500$, respectively. A particular behavior is displayed 
for $R e_{D}^{\prime}=1000$, a vortex structure is formed at the upper corner, and at the lower corner there is a pattern of a very small vortex in coexistence with an unyielded region with a $A_{T r} \approx 3.75 \times 10^{-4} D^{2}$. The coexistence of these structures is a result of the asymmetric behavior presented by the viscoplastic fluids for $R e_{D}^{\prime}$ near to the transition regime, hence the flow affects the behavior of the upper corner.

Figure 5.25 shows the evolution of the corner region for fluids $30 \mathrm{~W}$ and $40 \mathrm{~W}$, with $\tau_{0}^{H}=2.82$ $\mathrm{Pa}$ and $\tau_{0}^{H}=6.99 \mathrm{~Pa}$. For a $\tau_{0}^{H}=2.82 \mathrm{~Pa}$, the triangular area related to the unyielded region is $A_{T r} \approx 9.45 \times 10^{-3} D^{2}$ for a $\operatorname{Re}_{D}^{\prime}=10$. Then, the area is reduced for $\operatorname{Re}_{D}^{\prime}=100$ and 500 to $A_{T r} \approx 4.99 \times 10^{-3} D^{2}$ and $A_{T r} \approx 2.76 \times 10^{-3} D^{2}$, respectively. For fluid $40 \mathrm{~W}$ the unyielded area at the corner area also reduced as the $R e_{D}^{\prime}$ is increased. The $A_{T r}$ varies from $0.23 \times 10^{-3} D^{2}$ to $9.80 \times 10^{-3} D^{2}$ and $5.50 \times 10^{-3} D^{2}$, for $R e_{D}^{\prime}=1,10$ and 100 . It is possible to observed that as the $R e_{D}^{\prime}$ is increased in order of $10^{1}$ the $A_{T r}$ is reduced by half for the fluids with higher $\tau_{0}^{H}$.

The unyielded regions at the corner region can be fitted in a triangular area, as exposed in the last paragraph. The unyielded area generates a reduction of the entrance angle to the contraction plane, increasing the effects of the contraction. It is possible to state that for higher $\tau_{0}^{H}$, a greater unyielded area is obtained and the flow is more sensitive to the contraction effects, which is displayed in an early increase of the centerline velocity along the dimensionless axial distance, shown in Fig. 5.21.

Finally, comparing the different vortex structure and unyielded region patterns shown in Figs. 5.24 and 5.25 it can be stated that in the range of the $R e_{D}^{\prime}$ evaluated and for the contraction ratio studied $\beta=1.85$, vortex formations occur for fluid with low $\tau_{0}^{H}$ at corners of the contraction. Also, it was possible to visualize conditions with the coexistence of a small vortex structure and unyielded regions. For fluids with high $\tau_{0}^{H}$ is depicted the behavior of the unyielded regions among the different $R e_{D}^{\prime}$ values imposed, which are reduced at least in a $50 \%$ for each increasing of $10^{1}$ of the $R e_{D}^{\prime}$. 
Figure 5.24 - Visualization at the corner of the contractions for different $R e_{D}^{\prime}$. The generation and growth of the vortex structures are displayed by vector maps for fluid $10 \mathrm{~W}$ in a), b), c), d), e), and for fluid 20W in figures f), g), h), i), and j). Red dashed-lines delimited the unyielded regions.
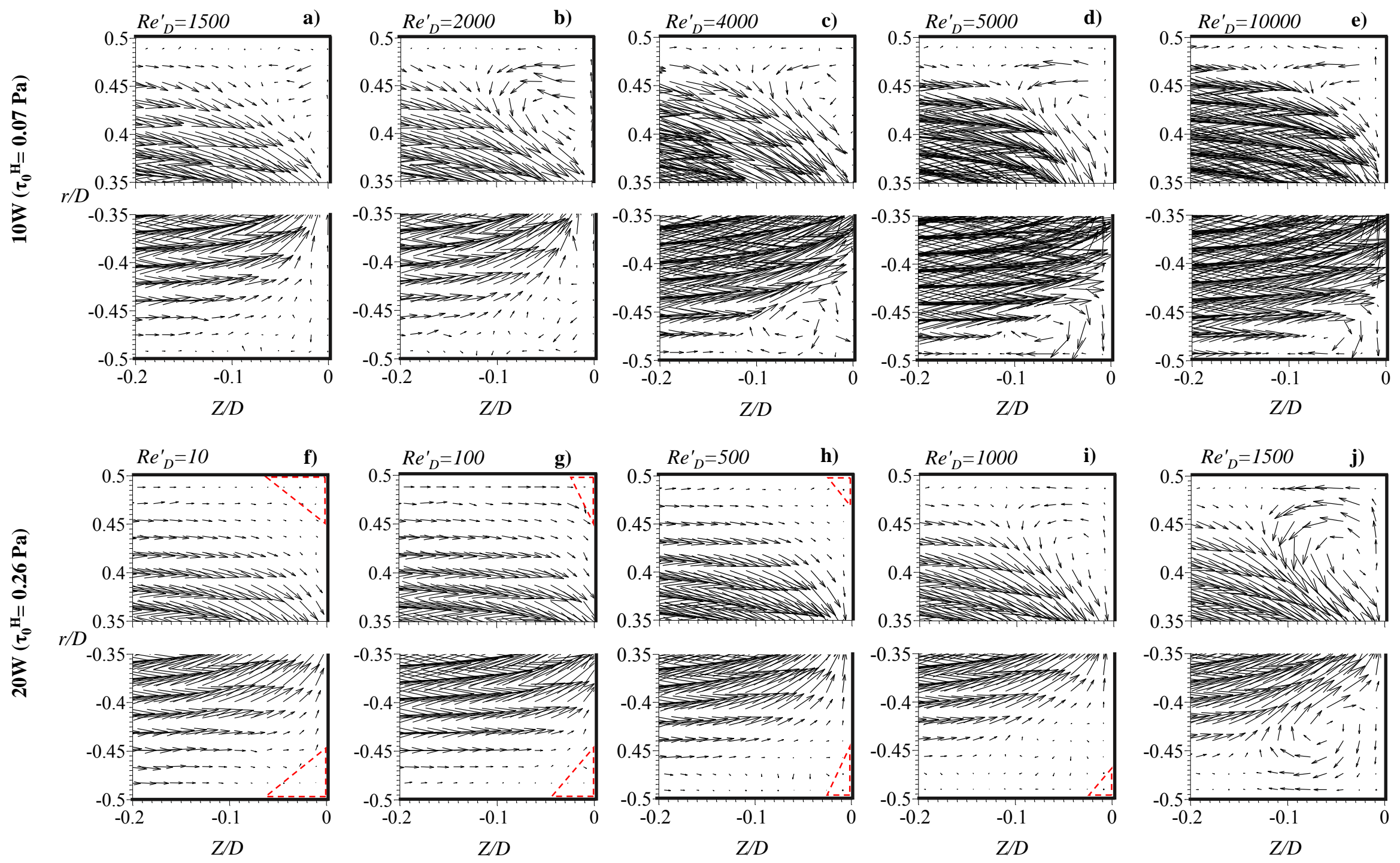

Source: Author (2019). 
Figure 5.25 - Visualization at the corner of the contractions for different $R e_{D}^{\prime}$. Unyielded regions are displayed by vector maps for fluid 40W in a), b), c), and for fluid 40W in figures d), e), and f). Red dashed-lines delimited the unyielded regions area.
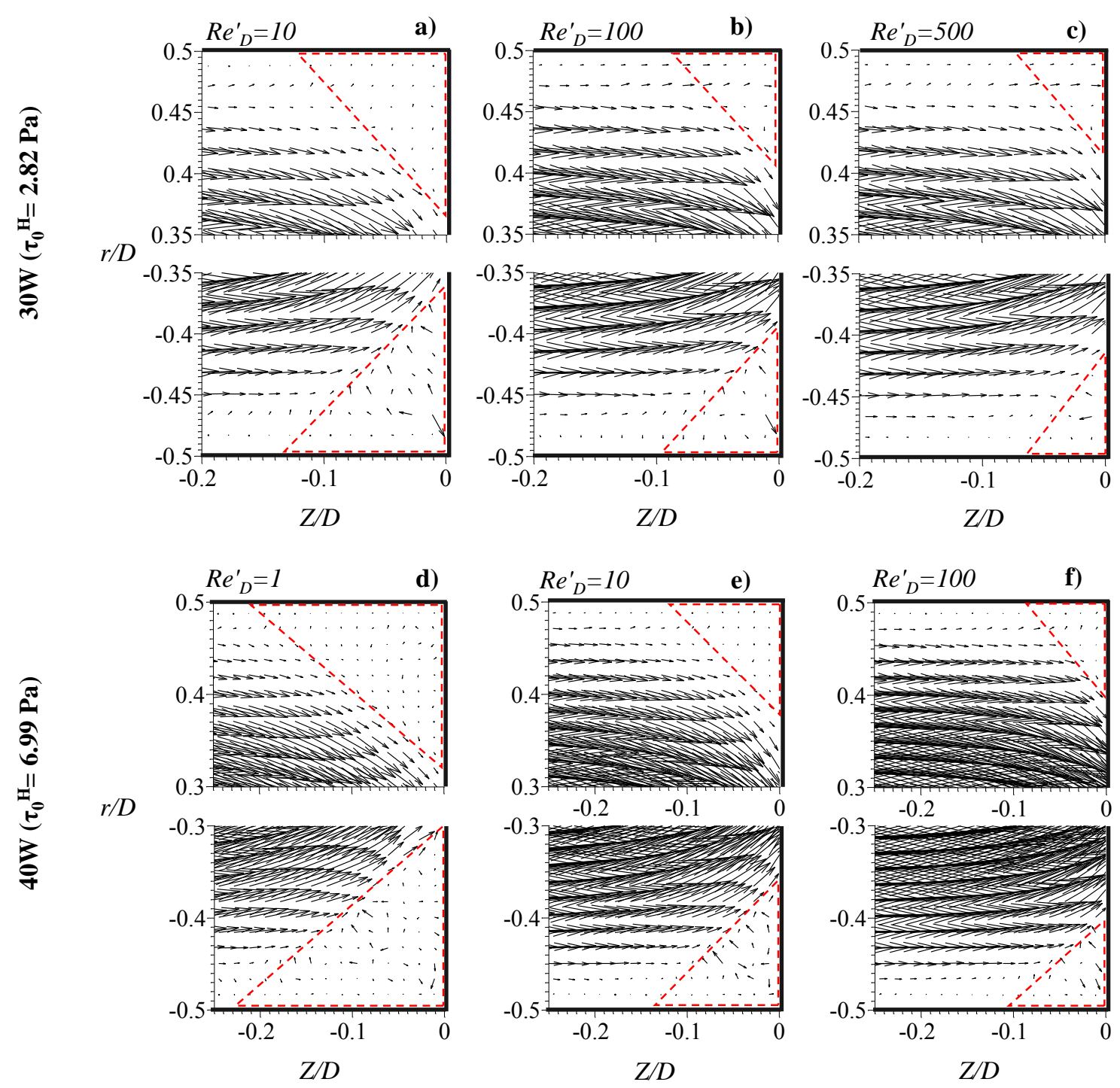

Source: Author (2019).

\subsubsection{Identification of Vortex Core and Vortex Core Center}

The experimental results for vortex structure are analyzed by quantitative criteria that allow to determine the size dimensions and vortex core position. The analysis of the vortex structures is based on vorticity limit values, pressure minima, closed pathlines, or streamlines. Jeong and Hussain (1995) covered different definitions and discussed the problem of using them to analyze the generation and behavior or vortices. Commonly, a vortex is associated with a region of the flow with high vorticity: nevertheless, there is no general verge over which the vorticity value can be considered high (HALLER, 2005), (PALACIOS-MORALES; ZENIT, 2013b). Then, the most widely used schemes to identify vortex structures are based on the local analysis of the velocity gradient tensor $\nabla \mathbf{u}$ (CHAKRABORTY et al., 2005). Some of these techniques based in the tensor invariant are the $Q$ criterion of Hunt et al. (1988), the 
$\lambda_{2}$ criterion of Jeong and Hussain (1995) and the $\Delta$ criterion proposed by Chong et al. (1990). A rational basis for vortex identification and general classification of 3D flow fields is provided by the velocity gradient decomposition given by:

$$
\nabla \mathbf{u}=\mathbf{S}+\mathbf{\Omega}
$$

where $\mathbf{S}$ and $\Omega$ are the symmetric (rate of strain tensor) and the antisymmetric (rate of rotation tensor) component of $\nabla \mathbf{u}$ given by:

$$
\begin{aligned}
& \mathbf{S}=\frac{1}{2}\left((\nabla \mathbf{u})+(\nabla \mathbf{u})^{T}\right) \\
& \boldsymbol{\Omega}=\frac{1}{2}\left((\nabla \mathbf{u})-(\nabla \mathbf{u})^{T}\right)
\end{aligned}
$$

\subsubsection{Lambda criterion}

The $\lambda_{2}$ criterion is based on the observation that the concept of a local pressure minimum in a plane fails to identify vortices under strong unsteady and viscous effects (CHAKRABORTY et al., 2005). Neglecting the unsteady and viscous effects, the symmetric part of the gradient of the incompressible Navier-Stokes equation can be expressed as:

$$
\mathbf{S}^{2}+\mathbf{\Omega}^{2}=-\frac{1}{\rho}(\nabla(\nabla p))
$$

where $p$ is the pressure and the Eq. (5.15) is a representation of the pressure Hessian $\left((\nabla(\nabla p))_{i j}=\right.$ $\left.\partial^{2} p / \partial x_{i} \partial x_{j}\right)$.

Jeong and Hussain (1995) define that the vortex core as a connected region with two positive eigenvalues of the pressure Hessian, and allows to capture the region of local pressure minimum in a plane. Since the tensor $\mathbf{S}^{2}+\Omega^{2}$ is real and symmetric all the three eigenvalues are real and they can be sorted so $\lambda_{1} \geq \lambda_{2} \geq \lambda_{3}$. If the point under analysis is part of a vortex at least two of these eigenvalues are negative, requiring simply $\lambda_{2}<0$. The eigenvalues of $\mathbf{S}^{\mathbf{2}}+\mathbf{\Omega}^{\mathbf{2}}$ and the $Q$ criterion are related by Jeong and Hussain (1995) in the following expression:

$$
\left.Q_{c}=-\frac{1}{2} \operatorname{tr}\left(\mathbf{S}^{2}+\mathbf{\Omega}^{\mathbf{2}}\right)=-\frac{1}{2}\left(\lambda_{1}+\lambda_{2}+\lambda_{3}\right)\right)
$$

where $Q_{c}>0$ due to $\lambda_{2}<0$, the local measure of rotation rate is larger than the strain rate; therefore, spatial region belong to a vortex (PALACIOS-MORALES; ZENIT, 2013b). It is important to highlight that while the $Q$ criterion measures the excess of rotation rate over the strain rate magnitude in al directions the $\lambda_{2}$ criterion looks for this excess at a specific point (JEONG; HUSSAIN, 1995), (CHAKRABORTY et al., 2005). For that reason the $\lambda_{2}$ criterion is used in the present study to evaluate and find the vortex core regions.

\subsubsection{Curvature method}

In a two-dimensional flow, it is possible to find points where the local velocity becomes zero (OUELLETTE; GOLLUB, 2007). These points are located in regions of the flow where the vorticity has a dominance, such points are elliptic; in a strain-dominated region, these points are hyperbolic as a saddle. 
Chong et al. (1990) demonstrated that elliptical points correspond to the center of the vortices in the flow, and it is possible to find these points by computing the curvature of the Lagrangian trajectories of the individual moving fluid elements. In the present study the velocity field obtained with the PIV technique is used to obtain the curvature points. In both cases the direction of the seeding particles in the fluid changes over very short length scales, producing large curvature values. Braun et al. (2006) proposed the scheme to obtain the curvature for a time-dependent flow, given by:

$$
k(t)=\frac{\left|\mathbf{u} \times \partial_{t} \mathbf{u}+\mathbf{u} \times[\mathbf{u} \cdot \nabla \mathbf{u}]\right|}{|\mathbf{u}|^{3}}
$$

where $\mathbf{u}$ is the velocity field and $\partial_{t}$ is the partial time derivate. The term $\mathbf{u} \times \partial_{t} \mathbf{u}$ can be obtained using central difference computation of three successive vector maps. However, due to the high rate of acquisition of the experiments $(50 \mathrm{~Hz})$, it is possible to compute the curvature without the temporal term obtaining a difference in the results lower than $0.8 \%$ (PALACIOS-MORALES; ZENIT, 2013b).

\subsubsection{Vortex core center position}

The position of the vortex core center is given for the dimensionless axial position $Z / D$, and the dimensionless radial position $r / D$. Then, the vortex core center position is identify for the upper corner as $Z / D_{v x_{u p}}$ and $r / D_{v x_{u p}}$, for the lower corner are identified by $Z / D_{v x_{d n}}$ and $r / D_{v x_{d n}}$.

Figure 5.26 shows the velocity, curvature, and the $\lambda_{2}$ criterion fields for fluid $10 \mathrm{~W}$ at the different $R e_{D}^{\prime}$ values set in this experimental study. The $\lambda_{2}$ criterion values are presented by gray-scale maps, the values vary according to the $R e_{D}^{\prime}$, for laminar conditions the maximum value is between -50 and -500 . According by the definition of Eq.(5.16), when the strain rate tensor excesses the rotation tensor the $\lambda_{2}$ value is 0 , and where there is a predominance of the rotation tensor there are negative values. The scalar maps were obtained after filtered the positive values to obtain a better contrast of the region affected by

vortex structures. When the $R e_{D}^{\prime}$ is increased, the values $\lambda_{2}$ increase for each fluid, but seems that there a reduction of the area with negative values.

The increase of the values for the eigenvalue of $\lambda_{2}$ is related to the increase of the rotation of the points inside of the vortex core. Then, it is possible to affirm that the vortex rotation increase with the $R e_{D}^{\prime}$. However, the vortex structures described by the $\lambda_{2}$ criterion shows that at the upper corner the vortex structure has an early appearance until the turbulent regime is achieved and the lower corner presents the highest values for the $\lambda_{2}$ eigenvalues. This change in the eigenvalues is related with the asymmetry behavior of the fluid flow for turbulent conditions, which also affects the evolution of the vortex structure formation.

The curvature contour is displayed by red lines that indicate the points with maximum curvature in the flow map. For each $R e_{D}^{\prime}$ the maximum points of curvature are marked by a black square; these points correspond to the core center of the vortex structure (points with elliptic curvature). It is possible to observe that the curvature method can be used together with $\lambda_{2}$ criteria to set the vortex center and the area under rotation. Nevertheless, for $R e_{D}^{\prime}=1500$ and $R e_{D}^{\prime}=10000$ the maximum curvature point is not inside the area delimited by the $\lambda_{2}$ criterion, this is due to the vortex core at these conditions is small. In these conditions, the eigenvalues show the area with maximum velocity gradients, and the center is 
Figure 5.26 - Comparison of the velocity field, lambda criterion and the curvature map for fluid 10W $\left(\tau_{0}^{H}=0.074 \mathrm{~Pa} ; m=0.002 \mathrm{~Pa} \cdot \mathrm{s}^{s} ; n=0.986\right)$ among the different $R e_{D}^{\prime}$ from laminar to turbulent regime. The curvature map is depicted by the red line contours, and the $\lambda_{2}$ criteria field is shown by a filled contour in gray scale. Black squares mark the position of the vortex structure center for each $R e_{D}^{\prime}$.
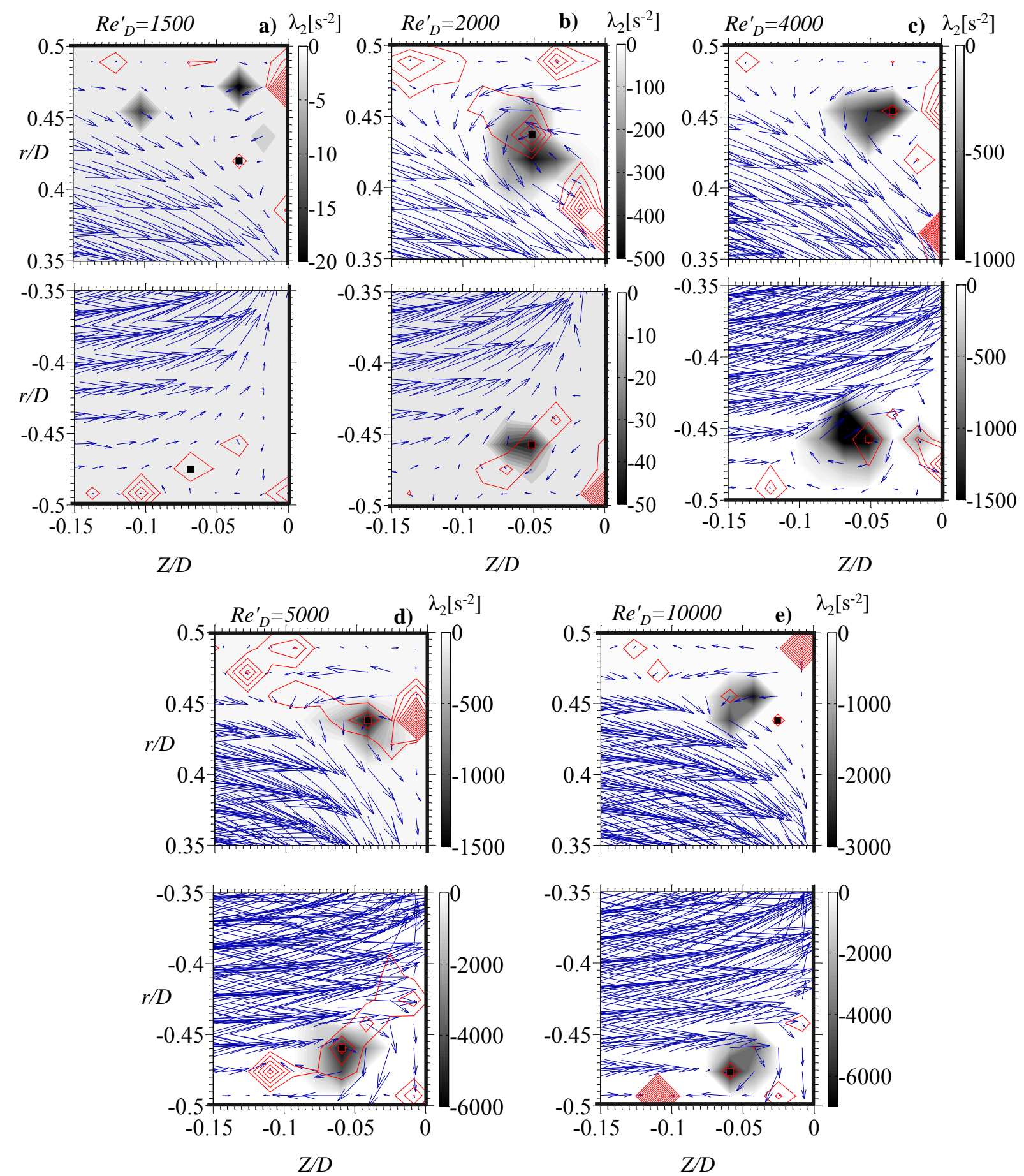

Source: Author (2019).

not included. The curvature method allows to find the vortex center and to define the shape of the vortex structures formed at the corners.

The table 5.3 shows the position for the vortex cores found by the curvature method. The vortex cores 
have a displacement in the vertical and horizontal position as the $R e_{D}^{\prime}$ is increased. At the upper corner, the vortex structure is close to the wall, as the $R e_{D}^{\prime}$ is increased the center moves backward. After the turbulent regime is achieved, the center position starts to approach the wall of the corner again. This behavior is due to the increase of the $R e_{D}^{\prime}$, and the vortex structures are pushed against the wall. On the other hand, the lower vortex structure presents a different behavior, for a $\operatorname{Re}_{D}^{\prime}=1500$ the center appears at $Z / D=-0.0687$ for a low rotation and moves forward to the wall and its axial position does not vary among the $R e_{D}^{\prime}$ when the turbulent regime is reached.

Table 5.3 - Position of the vortex structure for fluid $10 \mathrm{~W}$ at different $R e_{D}^{\prime}$

\begin{tabular}{l|l|l|l|l|l|l}
\hline & $R e_{D}^{\prime}$ & 1500 & 2000 & 4000 & 5000 & 10000 \\
\hline \multirow{2}{*}{ Upper } & $Z / D_{v x_{u p}}$ & -0.0342 & -0.0514 & -0.0342 & -0.0421 & -0.0252 \\
& $r / D_{v x_{u p}}$ & 0.4200 & 0.4371 & 0.4540 & 0.4381 & 0.4381 \\
\hline Below & $Z / D_{v x_{d n}}$ & -0.0687 & -0.0514 & -0.0514 & -0.0591 & -0.0591 \\
& $r / D_{v x_{d n}}$ & -0.4750 & -0.4575 & -0.4575 & -0.4593 & -0.4762 \\
\hline
\end{tabular}

Source: Author (2019).

For the vertical position is observed in Fig. 5.26 and table 5.3 that for $R e_{D}^{\prime}=4000$ and 5000 it seems to be symmetric behavior of the vortex structures with similar values between $Z / D_{v x_{u p}}$ and $Z / D_{v x_{d n}}$, before this $R e_{D}^{\prime}$ there an asymmetric due to the vortex structure at the lower corner is not completely formed. For a developed turbulent regime, the asymmetry effect of the flow modifies the position of the centers combined with the effect of the pressing by axial flow over the vortex.

On the other hand, Fig. 5.27 shows the $\lambda_{2}$ contour map and the curvature values for fluid 20W. As observed for fluid $10 \mathrm{~W}$, there is no symmetry of the vortices center due to the asymmetry condition of the flow for $R e_{D}^{\prime}$ near to the transition regime. For fluid $20 \mathrm{~W}$ the definition of the vortex structure is more clear than for fluid 10W; hence, higher $m$ and lower $n$ means a more viscous and stable fluid flow. The radial and axial position of the vortex center have low variations among the $R e_{D}^{\prime}$, contrary to the pattern observed for fluid 10W. The positions for the upper and lower vortices are summarized in table 5.4.

Table 5.4 - Position of the vortex structure for fluid $20 \mathrm{~W}$ at different $R e_{D}^{\prime}$

\begin{tabular}{l|l|l|l}
\hline & $R e_{D}^{\prime}$ & 1000 & 1500 \\
\hline \multirow{2}{*}{ Upper } & $Z / D_{v x_{u p}}$ & -0.0251 & -0.0419 \\
& $r / D_{v x_{u p}}$ & 0.4195 & 0.4195 \\
\hline Below & $Z / D_{v x_{d n}}$ & -0.0756 & -0.0756 \\
& $r / D_{v x_{d n}}$ & -0.4395 & -0.4395 \\
\hline
\end{tabular}

Source: Author (2019).

The center of the vortices have no variation in the radial position when the $R e_{D}^{\prime}$ is increased from 1500 to 2000, but in the axial direction there is a variation of the position; however, this variation in the axial position is lower than the presented for fluid 10W. In order to compare the behavior of the vortex structures positions in function of the $R e_{D}^{\prime}$, the Fig. 5.28 depicts these values for both vortex structures: upper $\left(v x_{u p}\right)$ and lower $\left(v x_{d n}\right)$. 
Figure 5.27 - Comparison of the velocity field, lambda criterion and the curvature map for fluid 20W $\left(\tau_{0}^{H}=0.262 \mathrm{~Pa} ; m=0.251 \mathrm{~Pa} \cdot \mathrm{s}^{s} ; n=0.634\right)$ among the different $R e_{D}^{\prime}$ from laminar to turbulent regime. The curvature map is depicts by the red line contours, and the $\lambda_{2}$ criteria field is shown by a filled contour in gray scale. Black squares mark the position of the vortex structure center for each $R e_{D}^{\prime}$.
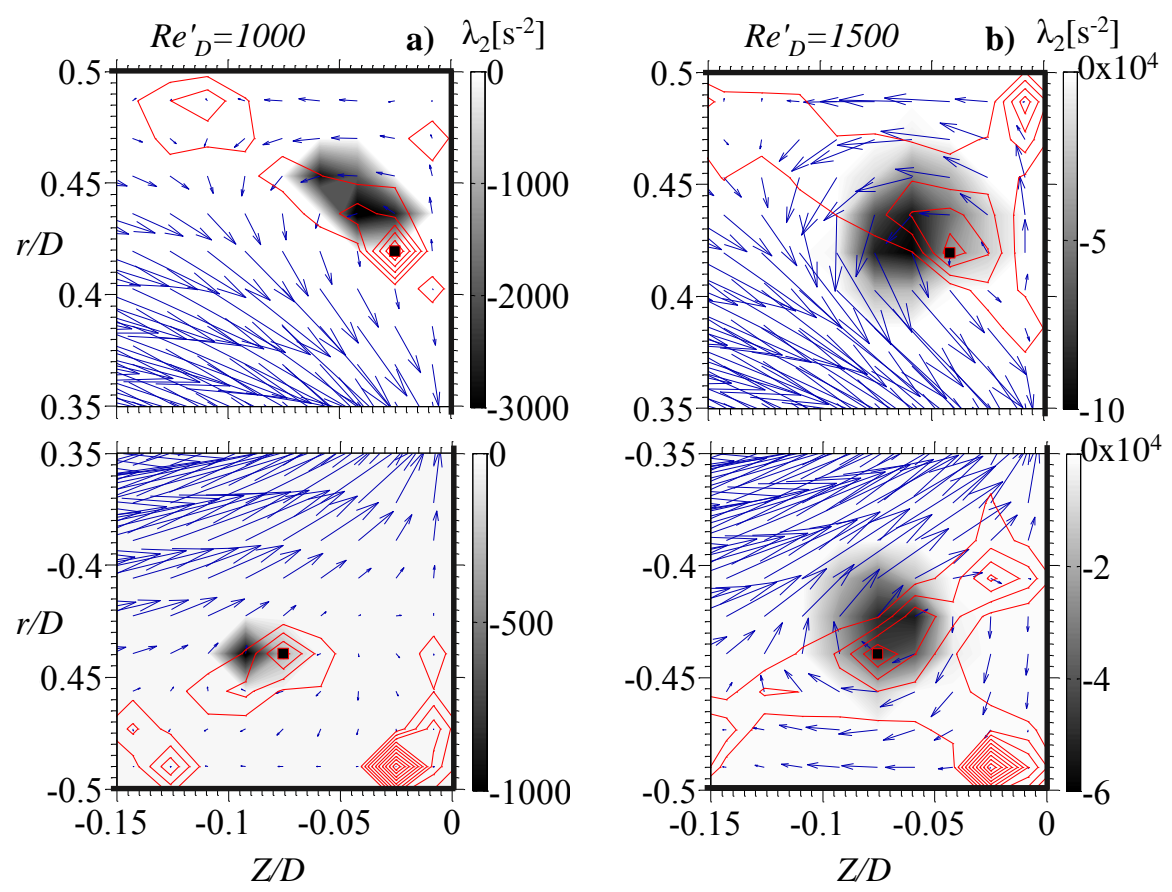

Source: Author (2019).

Figure 5.28 - Displacement of the vortex core center for fluids $10 \mathrm{~W}\left(H e_{H B} \approx 0 ; n=0.986\right)$ and $20 \mathrm{~W}$ $\left(H e_{H B}=2.938 ; n=0.634\right)$ as a function of the $R e_{D}^{\prime}$.
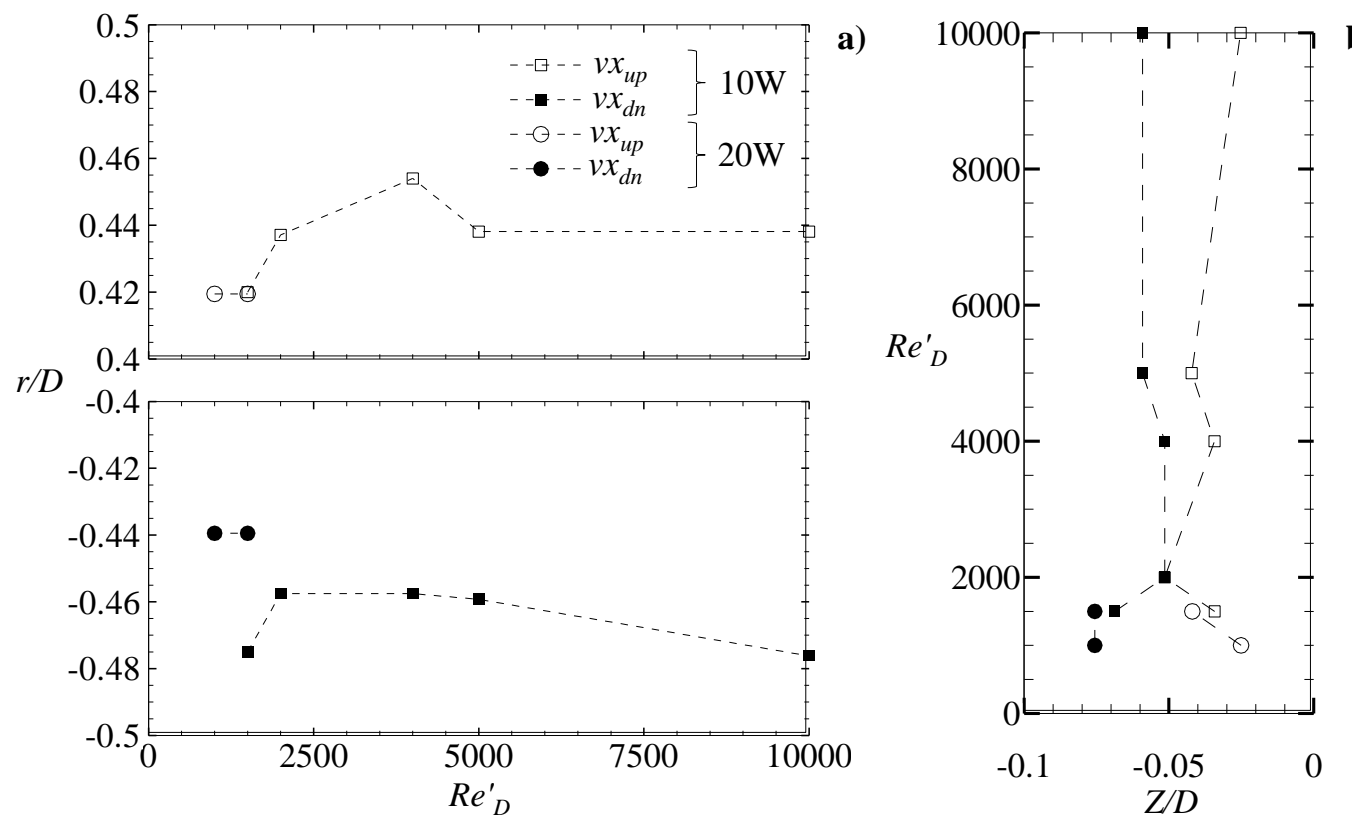

b)

Source: Author (2019). 
Figure 5.28a shows the displacement of the vortices at the upper and lower corner of the contraction. For fluid 10W with a low $H e_{H B}$ value, the radial position of the vortices varies in the laminar regime until a turbulent $R e_{D}^{\prime}$ is achieved, where the variation of the radial position decreases and the fluid tends to be closer to the walls. Also, a particular behavior is observed from Fig. 5.28a, the displacement of the upper and lower vortices along the radial coordinate for fluid $10 \mathrm{~W}$ is upward. Both vortex structures move in the same direction of the asymmetry flow observed in Fig. 5.7. The fluid $20 \mathrm{~W}$ does not present variation in the radial positions of the vortex structures, even though these are not in symmetry position relative to the centerline. It is possible to state that recirculation flow for fluid $20 \mathrm{~W}$ is more stable than the fluid $10 \mathrm{~W}$ due to a higher $m$ value, and lower $n$. Other, crucial aspect is that the circulation of the vortex core decreases with $n$, which means that lower rotations are obtained and, as a consequence, more stable and less sensitive to the increase of $R e_{D}^{\prime}$ in laminar conditions.

For the axial position of the vortex centers shown in Fig. 5.28 b, it is possible to observe that as for radial position the fluid $10 \mathrm{~W}$ presents variations among the $R e_{D}^{\prime}$, instead of fluid $20 \mathrm{~W}$ these variations are presented only for the upper vortex structure. For fluid $10 \mathrm{~W}$, these variations of position for the geometric centers of the vortices are similar to the variations in the radial direction, possibly influenced by the asymmetric condition presented in the turbulent regime. After the turbulent regime is achieved the vortex center approaches the wall $(Z / D=0)$, as discussed previously in this section: the flow presses the vortex structure against the corner wall as the $R e_{D}^{\prime}$ is increased.

It is important to note that the maximum displacement for both directions is $0.0172 \mathrm{D}$ for $10 \mathrm{~W}$ fluid and $0.0169 \mathrm{D}$ for fluid $20 \mathrm{~W}$, that is approximately $0.432 \mathrm{~mm}$, which is the length of an interrogation area side set for this experimental study, as described in the subsection 4.7.2. Therefore, there is an acceptable sensitivity of the grid as a result of the overlapping method used for the interrogation areas.

\subsubsection{Vortex core area}

The area influenced by the effect of the vortex structure can be calculated using the $\lambda_{2}$ criterion. After obtaining the scalar map for the $\lambda_{2}$ values, the distribution of the $\lambda_{2}$ values along the $Z / D$ position for each vortex is plotted. As the negative values indicated the presence of a region under the effect of a vortex area, and zero values refer to regions with a predominance of shear strain gradients.

Figures 5.29 and 5.30 show the distribution of the eigenvalues for each $R e_{D}^{\prime}$ for fluids $10 \mathrm{~W}$ and $20 \mathrm{~W}$. The values are plotted along the axial position of the each vortex center, identified as $Z / D_{v x_{u p}}$ and $Z / D_{v x_{d n}}$. The dashed horizontal lines show the radial coordinate of the vortex center.

The radial position of the vortex is inside the area delimited by the negative eigenvalues. However, Fig. 5.29a depicts the center vortex out of the $\lambda_{2}$ negative values area, as previously described in this subsection, this behavior is caused by the low rotation and the small size of the vortex. For this case, the core area of the vortex is considered until the vortex center. The area of vortex structures generated for fluid 10W grow in the laminar regime, and after the turbulent conditions are reached, the vortex core is reduced. In order to simplify the analysis for the core area, it is idealized a circular area that delimited the vortex core; therefore, from Figs. 5.29 and 5.30 are obtained the diameter for each vortex structure. Then, the ratio for the diameter of the vortex structures respect to the entrance diameter $\left(d_{v x} / D\right)$ for fluid $10 \mathrm{~W}$ increases from 0.0800 to 0.1250 for the upper vortex, and from 0.025 to 0.0875 in the laminar regime 
Figure 5.29 $-\lambda_{2}$ criteria eigenvalues along the position of the vortices center for fluid 10W $(n=0.986)$ at different $R e_{D}^{\prime}$. Dashed lines represent the radial position of the vortex center: red (upper vortex), and blue (lower vortex).
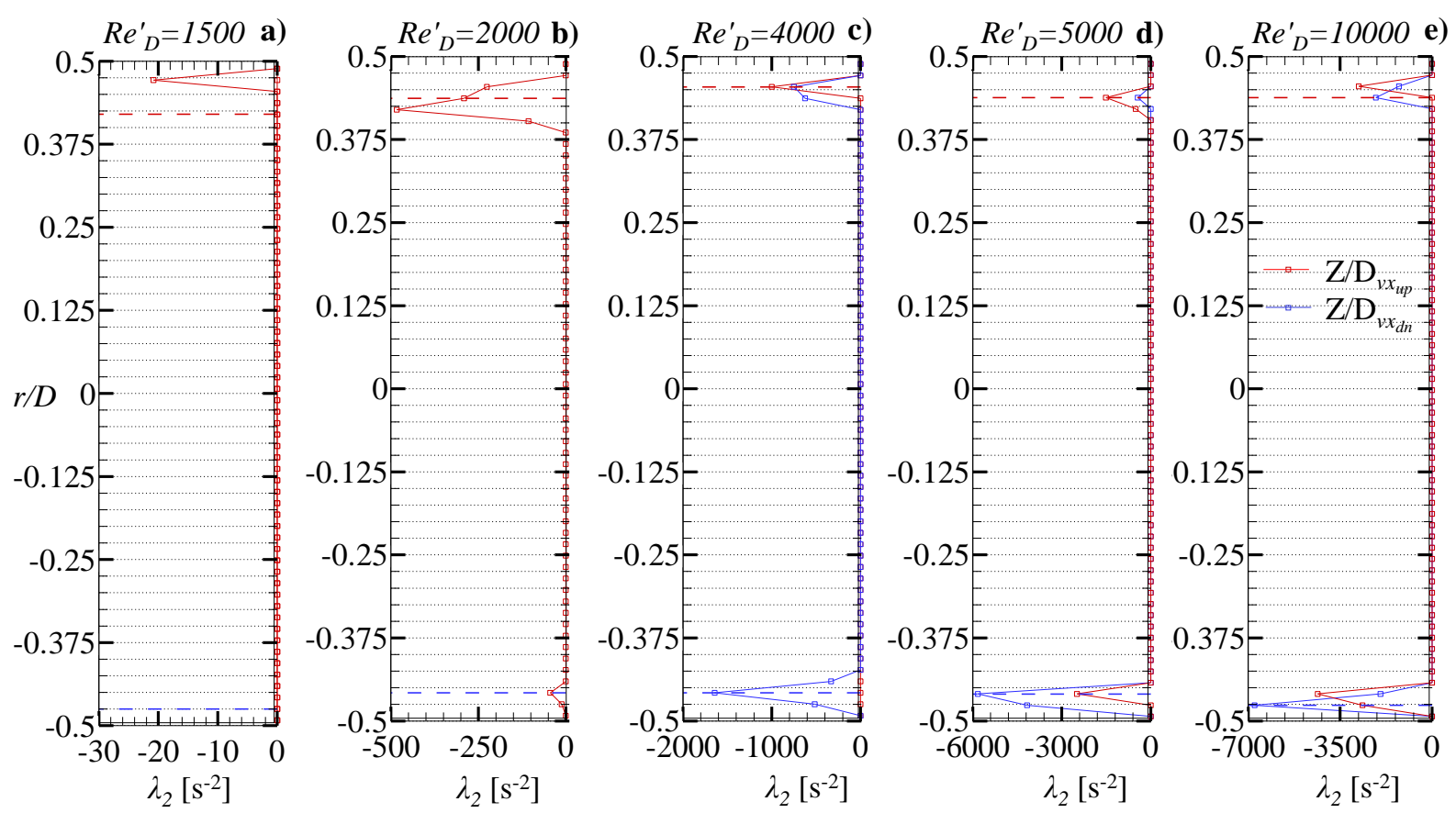

Source: Author (2019).

for $R e_{D}^{\prime}=1500$ and $R e_{D}^{\prime}=2000$, respectively. This growth of the vortex core is also observed for fluid $20 \mathrm{~W}$, which Fig. display that the ratio $d_{v x} / D$ for the upper vortex increases from 0.0850 to 0.1125 , and from 0.0750 to 0.1125 for the lower vortex core.

After the turbulent conditions are reached for fluid $10 \mathrm{~W}$, the ratio $d_{v x} / D$ decreases and reach a constant behavior. The Figure 5.31 shows the tends for the vortex core ratio for fluids $10 \mathrm{~W}$ and $20 \mathrm{~W}$ in function of the $R e^{\prime} D$. It is possible to state that the diameter of the vortex core decrease for turbulent regimes and tends to maintain a constant area. Palacios-Morales and Zenit (2013a) shows this dependence of the vortex core size and the $R e_{D}^{\prime}$ for laminar conditions in shear-thinning fluids, demonstrating a good agreement of the behavior described in the present study. Furthermore, the fluid $20 \mathrm{~W}$ presents higher $d_{v x} / D$ values than $10 \mathrm{w}$ for $R e_{D}^{\prime}=1500$. This behavior can be related to the shear-thinning properties; nevertheless, it is necessary more experimental data that were not covered in the present study to establish a correlation.

\subsection{AREA REDUCTIONS EFFECTS}

The abrupt area reduction leads to interesting effects on the fluid flow as described in the previous sections. As a case of study, in this section, it is analyzed the influence of the contraction on the variation for the Reynolds number at the entrance of the contraction for each viscoplastic fluid. It is possible to know the Reynolds number at the downstream section $\left(R e_{d}^{\prime}\right)$, using the continuity equation and using the 
Figure $5.30-\lambda_{2}$ criteria eigenvalues along the position of the vortices center for fluid 20W ( $n=0.634$ ) at different $R e_{D}^{\prime}$. Dashed lines represent the radial position of the vortex center: red (upper vortex), and blue (lower vortex).

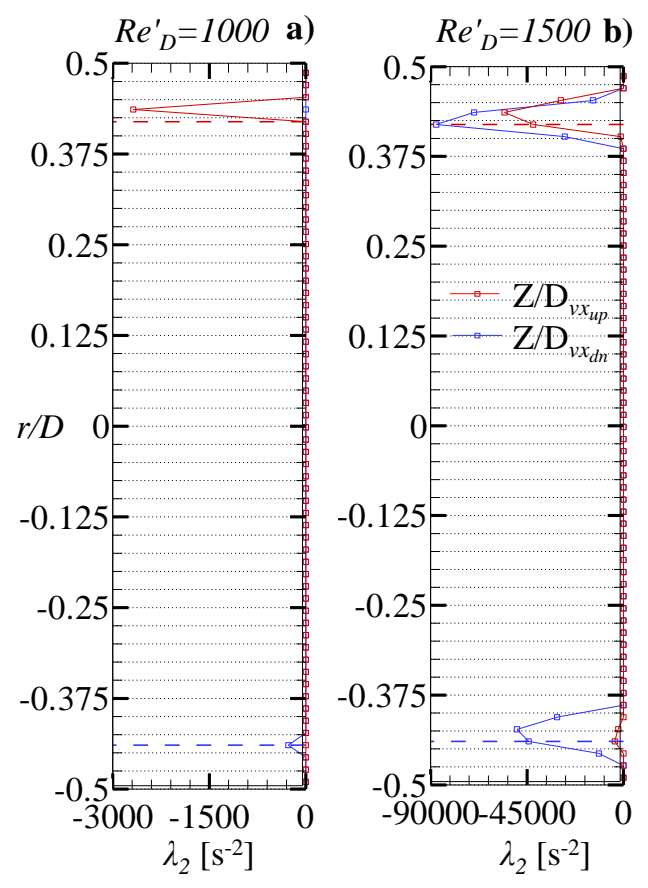

Source: Author (2019).

Figure 5.31 - Variation of the vortex core diameter for different $R e_{D}^{\prime}$. The upper and lower vortex core are presented for fluids $10 \mathrm{~W}$ and $20 \mathrm{~W}$.

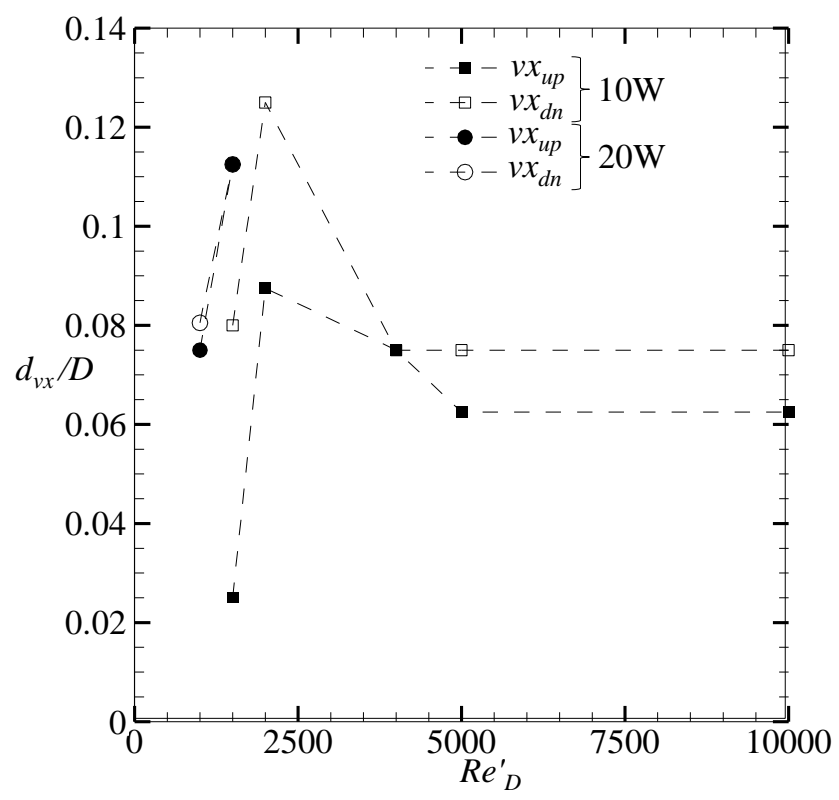

Source: Author (2019).

downstream diameter $d$. Therefore, it is possible to calculate the Reynolds number at the contraction plane $\left(R e_{d_{c}}^{\prime}\right)$, using the values obtained for the vortex diameters $\left(d_{v x}\right)$, and subtracting these diameters to predict an effective diameter at the entrance of the contraction $\left(d_{c}\right)$. For the cases with unyielded regions, it is 
possible to calculate this effective diameter using the major leg of the right-triangle $\left(L_{A_{T r}}\right)$ that delimits the unyielded area, see Figs.5.24 and 5.25.

Figure 5.32 presents a scheme describing the effective diameter at the entrance of the contraction, where $d_{c}=D-\left(d_{v x_{1}}+d_{v x_{2}}\right)$ for the cases with vortex structures and $d_{c}=D-\left(L_{A_{T r_{1}}}+L_{A_{T r_{2}}}\right)$ for the cases with unyielded regions at the corner of the contraction.

Figure 5.32 - Scheme of the effective diameter obtained by subtracting the vortex structure diameter or the major leg of the triangular unyielded region.

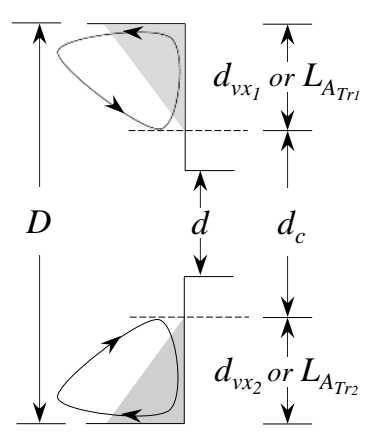

Source: Author (2019).

Figure 5.33a shows the variation of the ratio $d_{c} / D$ and the relation between $R e_{D}^{\prime}$ and $R e_{d_{c}}^{\prime}$. The ratio $d_{c} / D$ increases as the $R e_{D}^{\prime}$ is increased in the laminar region before the appearance of vortex structures. For the turbulent region, the ratio $d_{c} / D$ for fluid $10 \mathrm{~W}$ increases and seems to reach constant values. This behavior is related to the vortex core behavior described before in section 5.3.2, which reaches a constant behavior for turbulent $R e_{D}^{\prime}$. Also, it is possible to state that higher $H e_{H B}$ leads to a reduction of the effective diameter; hence the size of the unyielded regions at the corner of the contraction are greater and consequently represent an obstruction for the flow. However, the vortex structures at the $\operatorname{Re}_{D}^{\prime}$ for the present study have a more significant reduction of the effective diameter than the unyielded regions.

Figure 5.33b shows the correlation between $R e_{D}^{\prime}$ and $R e_{d_{c}}^{\prime}$. The decreasing of the effective diameter is compensated by the acceleration of the fluid, and higher velocities at the entrance section. Nevertheless, it is possible to observe that $R e_{d_{c}}^{\prime}$ is higher for fluids with a higher $H e_{H B}$ at the same $R e_{D}^{\prime}$. Fluid $40 \mathrm{~W}$ displays a $R e_{d_{c}}^{\prime}=29$ for a $R e_{D}=10$, instead fluid $30 \mathrm{~W}$ and $20 \mathrm{~W}$ display $R e_{d_{c}}^{\prime}=27$ and $R e_{d_{c}}^{\prime}=23$, respectively. As mentioned, an increase in the $\tau_{0}^{H}$ leads to greater unyielded regions at the corner, and these are deformed as the $R e_{D}^{\prime}$ is increased. However, due to the triangular profile of the toroid that described the unyielded volume at the entrance of the contraction, the base of this triangular profile is directly affected by the increase of the shear rate; while the side of the toroid at the contraction plane is little affected. For that reason, the effective diameter at the entrance of the contraction for fluids $30 \mathrm{~W}$ and $40 \mathrm{~W}$ are similar for laminar $R e_{D}^{\prime}$, obtaining similar values for $R e_{d_{c}}^{\prime}$.

Then, $R e_{D}^{\prime}$ and $R e_{d_{c}}^{\prime}$ depict a power-law correlation for the current geometry reduction $\beta=1.85$, as presented in Fig. 5.33b. This correlation is presented for the different viscoplastic fluids, where $R e_{d_{c}}^{\prime}$ is expressed as a function of $R e_{D}^{\prime}$ by:

$$
R e_{d_{c}}^{\prime}=2.894\left(\operatorname{Re}_{D}^{0.904}\right)
$$


Figure 5.33 - Behavior of the effective diameter ratio for the different fluids a). Reynolds number at the contraction plane, $R e_{d_{c}}^{\prime}$, in function of the $R e_{D}^{\prime}$.
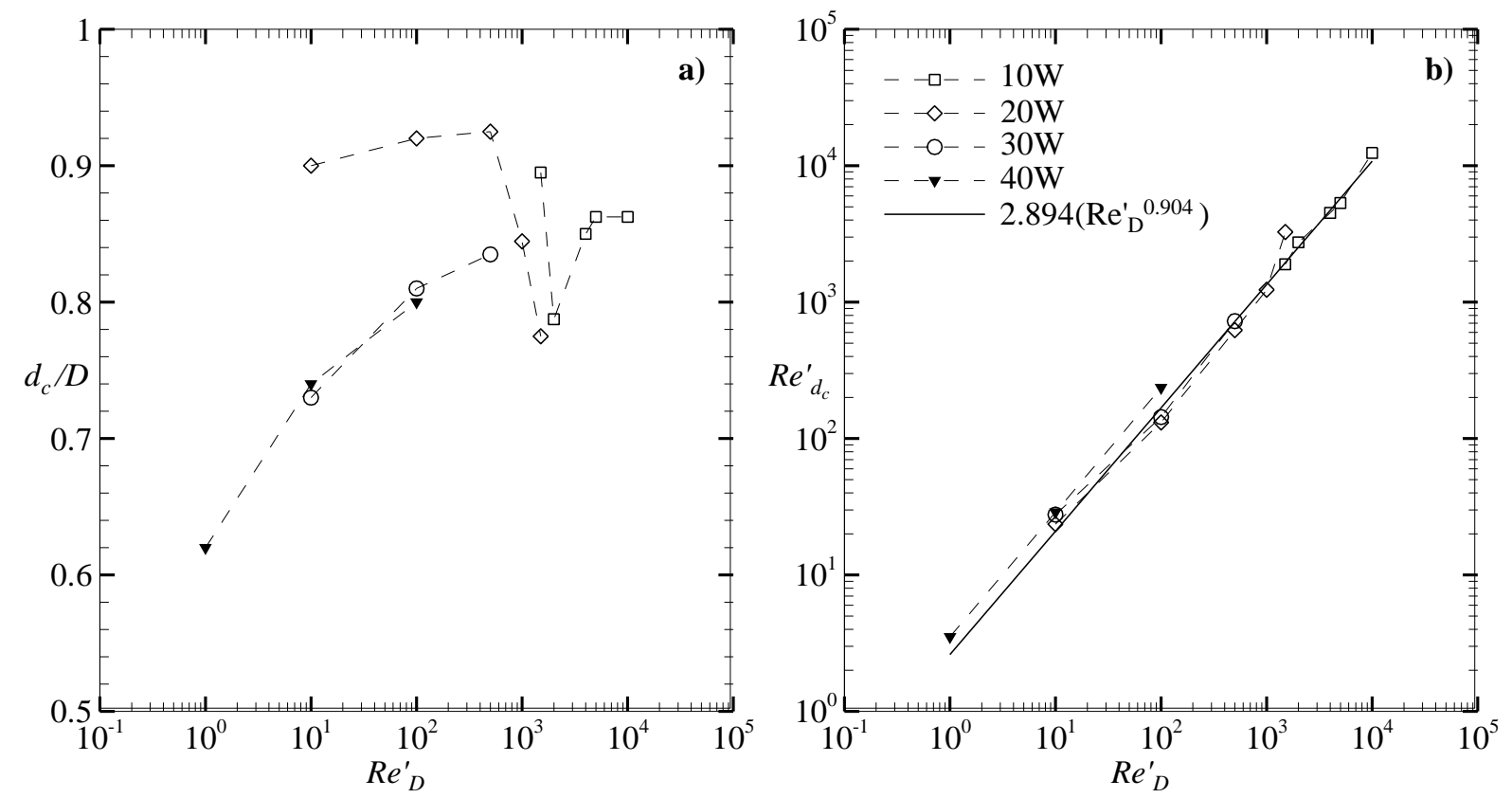

Source: Author (2019).

Nevertheless, it is necessary to state that Eq. (5.18) is a fit just for the conditions presented in this study. It is necessary test other $\beta$ configurations to find a general correlation for Herschel-Bulkley fluids.

Finally, it is possible to conclude that fluids with low $H e_{H B}$ are more suitable to be used for applications where a maximum effective diameter is required, due to in geometries with abrupt area reduction, small unyielded regions are generated for laminar conditions. For conditions with vortex structures generation, there are small areas under the effects of these recirculation flows for fluids with low $H e_{H B}$ values. Nevertheless, it is essential to note that a circulation analysis is required to study the effects of the area reduction in the circulation of the vortex structures, as stated by Palacios-Morales and Zenit (2013a), the circulation is higher for high $n$, and this decrease with $n$. And, small vortex with a high circulation can affect the effective flow going through the contraction. 


\section{CONCLUSIONS}

This project analyzes the flow of a viscoplastic fluid through an abrupt axisymmetric contraction with a ratio of $\beta=1.85$. The laminar and turbulent regimes are studied with Reynolds numbers at the entrance of the test section of $R e_{D}^{\prime}=1$ to 2000 , and 4000 to 10000 .

As part of the experimental methodology a flow loop was designed and built, and the PIV technique was used as a visualization technique. The methodology was tested with Newtonian fluid flow in a straight pipe for Reynolds number at the turbulent regime. The data obtained were compared and validated with numerical data and other experimental results available in the literature. A commercial ultrasound gel composed of Carbopol ${ }^{\circledR} 940$ in different concentrations was used as viscoplastic fluid. Rheological tests were performed to obtain shear rate-shear stress and shear rate-apparent viscosity curves. The data obtained were well fitted and represented by the Herschel-Bulkley model, which exhibits yield stress $\tau_{0}^{H}$, a coefficient of consistency $m$ and a shear-thinning index behavior $n$ variables. These rheological parameters can be correlated with the $R e_{D}^{\prime}$ using the definition of the Hedström number for Herschel-Bulkley fluids $H e_{H B}$.

The performance of the present experimental study was divided into two parts. First, the static pressure along the flow-loop was measured in order to calculate the pressure loss coefficient at the contraction as a function of the Reynolds number. Also, a correlation was obtained for the data obtained and compared with other numerical and experimental data for the behavior of the pressure loss coefficient for viscoplastic fluid flow. The second part of this experimental study is focused on the visualization of the fluid and with the assistance of the software Dynamic Studio at the upstream region of the contraction. The statistic flow maps were obtained, and from them were obtained the axial and radial velocities, also as the axial and radial velocities fluctuations for the turbulent regime.

The pressure drop at the contraction $\Delta P_{c o n}$ was obtained, extrapolating the static pressure profiles at the upstream and downstream regions of the contraction. Then, the dimensionless pressure drops $\Delta P_{c o n}^{\prime}$ displayed a decreasing for the laminar regime and reaches a constant value for the turbulent Reynolds number, $R e_{D}^{\prime}>2000$. The data obtained for the viscoplastic fluids were compared with the Newtonian case. It was noted that the values for $\Delta P_{c o n}^{\prime}$ are higher for viscoplastic fluids and it is related by the rheological properties, where the presence of the yield stress $\tau_{0}^{H}$ leads to higher pressure requirements at the entrance and consequently a higher drop of the static pressure at the contraction.

For the pressure loss coefficient $k_{c o n}$, similar behavior of the $\Delta P_{c o n}^{\prime}$ was obtained. A decrease is depicted for the laminar $R e_{D}^{\prime}$, and constant value is reached for turbulent conditions. The experimental data for $k_{c o n}$ were fitted using the K's method, obtaining the correlation $k_{c o n}=\frac{338}{R e_{D}^{\prime}}+1.38$. This correlation shows that there is no-dependence of the rheological parameters for $k_{c o n}$, this can be expressed as a simple function of the $R e_{D}^{\prime}$.

For the measurements with the PIV technique, the statistic vector maps display a particular behavior of the flow structure for viscoplastic fluids. An asymmetric behavior was observed for $R e_{D}^{\prime}$ near to the transitional regime for fluids with the lowest ultrasound gel concentrations. 
The axial velocity at the entrance of the test section for laminar conditions decreases as the $\tau_{0}^{H}$ increase for the viscoplastic solutions. For fluids $10 \mathrm{~W}$ and $20 \mathrm{~W}$ the ratio displays values of $u / \bar{U}=2$, instead for fluids $30 \mathrm{~W}$ and $40 \mathrm{~W}$ present values between 1.5 and 1.8. Also, for the laminar conditions as expected were obtained the higher values for the dimensionless axial velocity at the entrance of the contraction with values approximately $u / \bar{U}=3.5$. For turbulent conditions the values for the ratio decreases to $u / \bar{U}=2.8$. For the radial component, low values are obtained far from the contraction $v / \bar{U} \approx 0$, after the flow reaches the contraction, peak values are displayed. These peak values presented a low decrease as the $R e_{D}$ was increased, reaching values of $v / \bar{U}=0.8$. The data obtained for the dimensionless axial and radial velocities were compared with Newtonian fluid flow case; the viscoplastic fluids are less sensitive to the effects of the contraction due to Newtonian fluids the $u / \bar{U}$ and $v / \bar{U}$ presented similar values to the viscoplastic case both near to the contraction plane there is a significant difference that is related with the presence of the $\tau_{0}^{H}$.

For turbulent conditions lower values for the ratio $u / \bar{U}$ at the entrance of the test section were obtained. Ratio values of $u / \bar{U} \approx 1$, hence for turbulent conditions, a flat shape profile is displayed. Furthermore, as the behavior observed in the laminar conditions, the ratio value increase near the contraction plane. For the radial component, there is a similar behavior and values close to 0.8 ; nevertheless, near to the contraction, the $v / \bar{U}$ does not present peak value, there is a rounded shape of the profiles. Also, profiles for the turbulent regime were compared with the Newtonian case, and as the laminar case, the viscoplastic fluids exhibit lower values.

Comparing both regimes through the dimensionless centerline velocity $u_{C L} / \bar{U}$ is observed a similar behavior,

higher $u_{C L} / \bar{U}$ values at the contraction plane. Also, a particular behavior is presented by the viscoplastic fluids $30 \mathrm{~W}$ and $40 \mathrm{~W}$; the effects of the contraction start from a dimensional axial distance of $Z / D=-0.9$, instead of the other fluids the effects are displayed after $Z / D=-0.6$. This behavior is explained and complemented with the analysis of the unyielded regions at the corner of the contraction; due to high $\tau_{0}^{H}$ leads to greater unyielded areas, modifying the entrance geometry of the contraction and the fluid experiences early contractions effects.

From the analysis of the turbulent fluctuations for the axial and the radial velocities is observed that the fluctuations ratios $u^{\prime} / \bar{U}$ and $v^{\prime} / \bar{U}$ present maximum values near to the pipe wall. The lowest values are depicted in the central region. After the flow is near the contraction, these ratios display high fluctuations and reach values near to 0.4 . The profiles for $u^{\prime} / \bar{U}$ and $v^{\prime} / \bar{U}$ are compared with DNS data obtained for Newtonian and Herschel-Bulkley fluids. After the comparison, it was possible to state that for both type of fluids the values are similar, although for viscoplastic fluid the values are slightly higher, and the influence of the yield stress can be associated with higher values at the wall region.

The corners of the contraction were visualized and analyzed through the PIV technique. The unyielded regions at the corners increase with the $\tau_{0}^{H}$ value and decrease with the increasing of $R e_{D}^{\prime}$. The area of the unyielded regions was calculated by delimiting the area with the spurious vector at the corners that do not follow the behavior of the neighborhood, for fluid $20 \mathrm{~W}$ was possible to observe, for a $R e_{D}^{\prime}$, the coexistence of a small unyielded region and the formation of vortex structure. For fluids $30 \mathrm{~W}$ and $40 \mathrm{~W}$, the unyielded regions are at least $40 \%$ of the pipe diameter, representing a reduction of the effective flow 
diameter.

The vortex structures were identified and quantified using the $\lambda_{2}$ criterion, and the curvature method to find the center of the vortex core. It was possible to show that this methodology is precise to describe the behavior of the vortex structure and obtain quantitative data. As the $\operatorname{Re}_{D}^{\prime}$ was increased, the vortex structures presented reduction of their areas occupied by the cores. That means that the flow is pushed against the walls, and the cores are reduced, obtaining small structures but with higher circulation.

The effective diameter at the entrance of the contraction was calculated $d_{c}$, and a new Reynolds number at this region was obtained $R e_{d_{c}}^{\prime}$. This new definition for Reynolds number was correlated with $R e_{D}^{\prime}$, and a linear dependence was found, also as a particular behavior was possible to state that the $R e_{d_{c}}^{\prime}$ has no dependence of the rheological parameters for the viscoplastic fluids. This behavior is related to the high shear rates generated at the entrance of the contraction, where the fluid reaches conditions of constant viscosity.

Finally, this experimental study demonstrated that it is possible to obtain relevant information about the behavior of complex fluids as viscoplastic ones. This information allows to reduce the gap of data available for viscoplastic fluids under such flow conditions. Also, it was possible to use some methodologies to obtain quantitative data from the vortex structures and their behavior. It proved a good accuracy of these methodologies for flow through contractions. This data is now available for validation of future numerical studies.

\subsection{LIMITATIONS}

The analysis of complex fluids as viscoplastic one means a difficult task. The high consistency of some solutions restricted to lead the flow to the turbulent regime. Higher pressures than those that experimental setup could withstand are required to flow the solutions with high $\tau_{0}^{H}$ to turbulent conditions, and an experimental flow loop with a more powerful pump is necessary. This restriction represented a limitation to compare more data for the turbulent regime and establish further correlations for this regime for the behavior of the static pressure and the flow structure.

\subsection{SUGGESTIONS FOR FUTURE RESEARCH}

For the flow of the viscoplastic fluid through an abrupt contraction, the following topics could be considered in future research:

- Perform test for fluid with similar rheological parameters as fluid 20W, 30W, and 40W in turbulent conditions in order to compare the behavior with fluid $10 \mathrm{~W}$ and obtain a solid database for this regime.

- Other contraction ratios can be studied to compare the effect of the geometrical conditions on the flow structure. Also, it is possible to obtain a correlation between $R e_{D}^{\prime}$ and $R e_{d_{c}}^{\prime}$ as a function of $\beta$. 
- Obtain the values for the circulation of the vortex structure and establish correlations as a function of the rheological parameters.

- Visualize the downstream region of the contraction to study the restructuring of the plug core at this region in the laminar regime.

- Determine from the data obtained the pressure maps, and study their relationship with the velocity changes in the region visualized. Also, it is possible to obtain viscosity and shear rate maps and attach the behavior of these variables with the pressure and velocity changes.

- Extend the PIV technique for studying the viscoplastic fluid flow through other geometries, and to other fluids with non-Newtonian properties as thixotropic materials and expand the understanding over these complex fluids. Also, to obtain information about phenomena that can not be studied due to the limitations of numerical studies.

Finally, as a general suggestion, the behavior of other non-Newtonian materials can be visualized and analyzed using PIV measurements, increasing the experimental data about the behavior of these materials under certain conditions, and can be used as validation for numerical studies. 


\section{BIBLIOGRAPHY}

ABDALI, S.; MITSOULIS, E.; MARKATOS, N. Entry and exit flows of bingham fluids. Journal of Rheology, SOR, v. 36, n. 2, p. 389-407, 1992.

ALEXANDROU, A. N.; MENN, P. L.; GEORGIOU, G.; ENTOV, V. Flow instabilities of herschel-bulkley fluids. Journal of Non-Newtonian Fluid Mechanics, Elsevier, v. 116, n. 1, p. 19-32, 2003.

ASTARITA, G.; GRECO, G. Excess pressure drop in laminar flow through sudden contraction. newtonian liquids. Industrial \& Engineering Chemistry Fundamentals, ACS Publications, v. 7, n. 1, p. 27-31, 1968.

BALMFORTH, N. J.; FRIGAARD, I. A.; OVARLEZ, G. Yielding to stress: recent developments in viscoplastic fluid mechanics. Annual Review of Fluid Mechanics, Annual Reviews, v. 46, p. 121-146, 2014.

BARNES, H.; WALTERS, K. The yield stress myth? Rheologica acta, Springer, v. 24, n. 4, p. 323-326, 1985.

BARNES, H. A. The yield stress-a review or ' $\pi \alpha \nu \tau \alpha \rho \varepsilon \iota$ ' - everything flows? Journal of Non-Newtonian Fluid Mechanics, Elsevier, v. 81, n. 1-2, p. 133-178, 1999.

BARNES, H. A. A handbook of elementary rheology. University of Wales, Institute of Non-Newtonian Fluid Mechanics Aberystwyth, 2000.

BARNES, H. A.; HUTTON, J. F.; WALTERS, K. An introduction to rheology. [S.1.]: Elsevier, 1989.

BIRD, R. B.; ARMSTRONG, R. C.; HASSAGER, O. Dynamics of polymeric liquids. vol. 1: Fluid mechanics. 1987.

BIRD, R. B.; DAI, G.; YARUSSO, B. J. The rheology and flow of viscoplastic materials. Reviews in Chemical Engineering, De Gruyter, v. 1, n. 1, p. 1-70, 1983.

BLASIUS, H. Das aehnlichkeitsgesetz bei reibungsvorgängen in flüssigkeiten. In: Mitteilungen über Forschungsarbeiten auf dem Gebiete des Ingenieurwesens. [S.1.]: Springer, 1913. p. 1-41.

BOERSMA, W. H.; LAVEN, J.; STEIN, H. N. Shear thickening (dilatancy) in concentrated dispersions. AIChE journal, Wiley Online Library, v. 36, n. 3, p. 321-332, 1990.

BOGER, D.; CROCHET, M.; KEILLER, R. On viscoelastic flows through abrupt contractions. Journal of non-newtonian fluid mechanics, Elsevier, v. 44, p. 267-279, 1992.

BONN, D.; DENN, M. M.; BERTHIER, L.; DIVOUX, T.; MANNEVILLE, S. Yield stress materials in soft condensed matter. Reviews of Modern Physics, APS, v. 89, n. 3, p. 035005, 2017.

BRAUN, W.; LILLO, F. D.; ECKHARDT, B. Geometry of particle paths in turbulent flows. Journal of Turbulence, Taylor \& Francis, n. 7, p. N62, 2006.

CARR, Z.; AHMED, K.; FORLITI, D. Spatially correlated precision error in digital particle image velocimetry measurements of turbulent flows. Experiments in fluids, Springer, v. 47, n. 1, p. 95-106, 2009.

CHAKRABORTY, P.; BALACHANDAR, S.; ADRIAN, R. J. On the relationships between local vortex identification schemes. Journal of fluid mechanics, Cambridge University Press, v. 535, p. 189-214, 2005.

CHENG, D. C. Yield stress: a time-dependent property and how to measure it. Rheologica Acta, Springer, v. 25 , n. 5 , p. $542-554,1986$. 
CHHABRA, R. P.; RICHARDSON, J. F. Non-Newtonian flow and applied rheology: engineering applications. [S.1.]: Butterworth-Heinemann, 2011.

CHONG, M. S.; PERRY, A. E.; CANTWELL, B. J. A general classification of three-dimensional flow fields. Physics of Fluids A: Fluid Dynamics, AIP, v. 2, n. 5, p. 765-777, 1990.

CHRISTENSEN, K. The influence of peak-locking errors on turbulence statistics computed from piv ensembles. Experiments in Fluids, Springer, v. 36, n. 3, p. 484-497, 2004.

COLEMAN, H. W.; STEELE, W. G. Experimentation, validation, and uncertainty analysis for engineers. [S.1.]: John Wiley \& Sons, 2018.

COUPEZ, T.; ZINE, M.; AGASSANT, J. Numerical simulation of bingham fluid flow. In: Proceedings of the 4th European Rheology Conference, Seville. [S.1.: s.n.], 1994. p. 341-343.

COUSSOT, P.; MALKIN, A. Y.; OVARLEZ, G. Introduction: yield stress-or 100 years of rheology. [S.1.]: Springer, 2017.

COUSSOT, P.; NGUYEN, Q.; HUYNH, H.; BONN, D. Viscosity bifurcation in thixotropic, yielding fluids. Journal of rheology, SOR, v. 46, n. 3, p. 573-589, 2002.

CURRAN, S.; HAYES, R.; AFACAN, A.; WILLIAMS, M.; TANGUY, P. Properties of carbopol solutions as models for yield-stress fluids. Journal of food science, Wiley Online Library, v. 67, n. 1, p. 176-180, 2002.

DESHPANDE, A. P. Oscillatory shear rheology for probing nonlinear viscoelasticity of complex fluids: Large amplitude oscillatory shear. In: Rheology of complex fluids. [S.1.]: Springer, 2010. p. 87-110.

DESHPANDE, A. P. Piv techniques in experimental measurement of two phase (gas-liquid) systems. In: Rheology of complex fluids. [S.1.]: Springer, 2010. p. 111-129.

DODGE, D.; METZNER, A. Turbulent flow of non-newtonian systems. AIChE Journal, Wiley Online Library, v. 5, n. 2, p. 189-204, 1959.

DOORNE, C. V.; WESTERWEEL, J. Measurement of laminar, transitional and turbulent pipe flow using stereoscopic-piv. Experiments in Fluids, Springer, v. 42, n. 2, p. 259-279, 2007.

DUDA, J.; VRENTAS, J. Entrance flows of non-newtonian fluids. Transactions of the Society of Rheology, SOR, v. 17, n. 1, p. 89-108, 1973.

DURST, F.; JOVANOVIĆ, J.; SENDER, J. Lda measurements in the near-wall region of a turbulent pipe flow. Journal of Fluid Mechanics, Cambridge University Press, v. 295, p. 305-335, 1995.

DURST, F.; LOY, T. Investigations of laminar flow in a pipe with sudden contraction of cross sectional area. Computers \& fluids, Elsevier, v. 13, n. 1, p. 15-36, 1985.

DYNAMICS, D. Measurement principles of piv. https://www.dantecdynamics.com/measurementprinciples-of-piv, 2015. Accessed 29-08-2018.

DZUY, N. Q.; BOGER, D. V. Yield stress measurement for concentrated suspensions. Journal of Rheology, SOR, v. 27, n. 4, p. 321-349, 1983.

EGGELS, J.; UNGER, F.; WEISS, M.; WESTERWEEL, J.; ADRIAN, R.; FRIEDRICH, R.; NIEUWSTADT, F. Fully developed turbulent pipe flow: a comparison between direct numerical simulation and experiment. Journal of Fluid Mechanics, Cambridge University Press, v. 268, p. 175-210, 1994. 
ELGHOBASHI, S. On predicting particle-laden turbulent flows. Applied scientific research, Springer, v. 52, n. 4, p. 309-329, 1994.

ESCUDIER, M.; PRESTI, F. Pipe flow of a thixotropic liquid. Journal of Non-Newtonian Fluid Mechanics, Elsevier, v. 62, n. 2-3, p. 291-306, 1996.

FALL, A.; HUANG, N.; BERTRAND, F.; OVARLEZ, G.; BONN, D. Shear thickening of cornstarch suspensions as a reentrant jamming transition. Physical Review Letters, APS, v. 100, n. 1, p. 018301, 2008.

FALL, A.; LEMAITRE, A.; BERTRAND, F.; BONN, D.; OVARLEZ, G. Shear thickening and migration in granular suspensions. Physical review letters, APS, v. 105, n. 26, p. 268303, 2010.

FERNANDES, R. R.; TUREZO, G.; ANDRADE, D. E.; FRANCO, A. T.; NEGRÃO, C. O. R. Are the rheological properties of water-based and synthetic drilling fluids obtained by the fann 35a viscometer reliable? Journal of Petroleum Science and Engineering, Elsevier, v. 177, p. 872-879, 2019.

FESTER, V.; MBIYA, B.; SLATTER, P. Energy losses of non-newtonian fluids in sudden pipe contractions. Chemical Engineering Journal, Elsevier, v. 145, n. 1, p. 57-63, 2008.

FRITZ, G.; MARANZANO, B.; WAGNER, N.; WILLENBACHER, N. High frequency rheology of hard sphere colloidal dispersions measured with a torsional resonator. Journal of non-newtonian fluid mechanics, Elsevier, v. 102, n. 2, p. 149-156, 2002.

FROISHTETER, G.; VINOGRADOV, G. The laminar flow of plastic disperse systems in circular tubes. Rheologica Acta, Springer, v. 19, n. 2, p. 239-250, 1980.

GANS, R. F. On the flow of a yield strength fluid through a contraction. Journal of non-newtonian fluid mechanics, Elsevier, v. 81, n. 3, p. 183-195, 1999.

GIUSEPPE, E. D.; CORBI, F.; FUNICIELLO, F.; MASSMEYER, A.; SANTIMANO, T.; ROSENAU, M.; DAVAILLE, A. Characterization of carbopol ${ }^{\circledR}$ hydrogel rheology for experimental tectonics and geodynamics. Tectonophysics, Elsevier, v. 642, p. 29-45, 2015.

GOVIER, G. W.; AZIZ, K. The flow of complex mixtures in pipes. [S.1.]: Van Nostrand Reinhold Company New York, 1972. v. 469.

HALLER, G. An objective definition of a vortex. Journal of fluid mechanics, Cambridge University Press, v. 525, p. 1-26, 2005.

HAMED, S. B.; BELHADRI, M. Rheological properties of biopolymers drilling fluids. Journal of Petroleum Science and Engineering, Elsevier, v. 67, n. 3-4, p. 84-90, 2009.

HAMMAD, K. J.; OTUGEN, M. V.; VRADIS, G. C.; ARIK, E. B. Laminar flow of a nonlinear viscoplastic fluid through an axisymmetric sudden expansion. Journal of fluids engineering, American Society of Mechanical Engineers, v. 121, n. 2, p. 488-495, 1999.

HAMMAD, K. J.; VRADIS, G. C. Creeping flow of a bingham plastic through axisymmetric sudden contractions with viscous dissipation. International journal of heat and mass transfer, Elsevier, v. 39, n. 8, p. $1555-1567,1996$.

HANKS, R. W. The laminar-turbulent transition for fluids with a yield stress. AIChE Journal, Wiley Online Library, v. 9, n. 3, p. 306-309, 1963.

HANKS, R. W. et al. On the flow of bingham plastic slurries in pipes and between parallel plates. Society of Petroleum Engineers Journal, Society of Petroleum Engineers, v. 7, n. 04, p. 342-346, 1967. 
HE, Q.; GONG, X.; XUAN, S.; JIANG, W.; CHEN, Q. Shear thickening of suspensions of porous silica nanoparticles. Journal of materials science, Springer, v. 50, n. 18, p. 6041-6049, 2015.

HEDSTRÖM, B. O. Flow of plastic materials in pipes. Industrial \& Engineering Chemistry, ACS Publications, v. 44, n. 3, p. 651-656, 1952.

HEYWOOD, N.; CHENG, D.-H. Comparison of methods for predicting head loss in turbulent pipe flow of non-newtonian fluids. Transactions of the Institute of Measurement and Control, Sage Publications Sage CA: Thousand Oaks, CA, v. 6, n. 1, p. 33-45, 1984.

HILL, R. 7" he Mathematical Theory of Plasticity. [S.1.]: Oxford University Press, 1950.

HOOPER, W. B. The 2-k method predicts head losses in pipe fittings. Chemical Engineering, MCGRAW HILL INC 1221 AVENUE OF THE AMERICAS, NEW YORK, NY 10020, v. 88, n. 17, p. 96-100, 1981.

HUNT, J. C.; WRAY, A. A.; MOIN, P. Eddies, streams, and convergence zones in turbulent flows. 1988.

ISAYEV, A.; HUANG, Y. Two-dimensional planar flow of a viscoelastic plastic medium. Rheologica acta, Springer, v. 32, n. 2, p. 181-191, 1993.

JAY, P.; MAGNIN, A.; PIAU, J. M. Numerical simulation of viscoplastic fluid flows through an axisymmetric contraction. Journal of fluids engineering, American Society of Mechanical Engineers, v. 124, n. 3, p. 700-705, 2002.

JEONG, J.; HUSSAIN, F. On the identification of a vortex. Journal of fluid mechanics, Cambridge University Press, v. 285, p. 69-94, 1995.

KEE, D. D.; DURNING, D. Rheology of materials with a yield stress. Polymer theology and processing. Elsevier, New York, p. 177-203, 1990.

KFURI, S. L.; SILVA, J. Q.; SOARES, E. J.; THOMPSON, R. L. Friction losses for power-law and viscoplastic materials in an entrance of a tube and an abrupt contraction. Journal of Petroleum Science and Engineering, Elsevier, v. 76, n. 3-4, p. 224-235, 2011.

KHANDAVALLI, S.; ROTHSTEIN, J. P. Large amplitude oscillatory shear rheology of three different shear-thickening particle dispersions. Rheologica Acta, Springer, v. 54, n. 7, p. 601-618, 2015.

KITTREDGE, H.; ROWLEY, R. Estimating friction loss coefficient. Journal of Fluid Mechanics, Cambridge University Press, v. 81, p. 133-178, 1957.

LIU, G.; LU, H. Laser-induced fluorescence of rhodamine b in ethylene glycol solution. Procedia engineering, Elsevier, v. 102, p. 95-105, 2015.

MADLENER, K.; FREY, B.; CIEZKI, H. Generalized reynolds number for non-newtonian fluids. Progress in Propulsion Physics, EDP Sciences, v. 1, p. 237-250, 2009.

MAGNIN, A.; PIAU, J. Flow of yield stress fluids through a sudden change of section. In: Theoretical and Applied Rheology. [S.1.]: Elsevier, 1992. p. 195-197.

MALIN, M. Turbulent pipe flow of herschel-bulkley fluids. International communications in heat and mass transfer, Pergamon, v. 25, n. 3, p. 321-330, 1998.

MALKIN, A. Y. Rheology fundamentals. [S.1.]: ChemTec Publishing, 1994.

MAO, X.; JAWORSKI, A. J. Application of particle image velocimetry measurement techniques to study turbulence characteristics of oscillatory flows around parallel-plate structures in thermoacoustic devices. Measurement Science and Technology, IOP Publishing, v. 21, n. 3, p. 035403, 2010. 
METZNER, A.; REED, J. Flow of non-newtonian fluids-correlation of the laminar, transition, and turbulent-flow regions. Aiche journal, Wiley Online Library, v. 1, n. 4, p. 434-440, 1955.

MEWIS, J.; SPAULL, A. Rheology of concentrated dispersions. Advances in Colloid and interface Science, Citeseer, v. 6, n. 3, p. 173-200, 1976.

MIKA, L. Energy losses of ice slurry in pipe sudden contractions. Experimental Thermal and Fluid Science, Elsevier, v. 35, n. 6, p. 939-947, 2011.

MITSOULIS, E.; ABDALI, S.; MARKATOS, N. Flow simulation of herschel-bulkley fluids through extrusion dies. The Canadian Journal of Chemical Engineering, Wiley Online Library, v. 71, n. 1, p. 147-160, 1993.

MØLLER, P.; FALL, A.; BONN, D. Origin of apparent viscosity in yield stress fluids below yielding. EPL (Europhysics Letters), IOP Publishing, v. 87, n. 3, p. 38004, 2009.

MØLLER, P. C.; MEWIS, J.; BONN, D. Yield stress and thixotropy: on the difficulty of measuring yield stresses in practice. Soft matter, Royal Society of Chemistry, v. 2, n. 4, p. 274-283, 2006.

MORRISON, F. A. Understanding Rheology, Topics in Chemical Engineering. [S.1.]: Oxford University Press, New York, 2001.

NA, W.; AHN, H.; HAN, S.; HARRISON, P.; PARK, J. K.; JEONG, E.; YU, W.-R. Shear behavior of a shear thickening fluid-impregnated aramid fabrics at high shear rate. Composites Part B: Engineering, Elsevier, v. 97, p. 162-175, 2016.

NGUYEN, H.; BOGER, D. The kinematics and stability of die entry flows. Journal of non-Newtonian fluid mechanics, Elsevier, v. 5, p. 353-368, 1979.

NIEUWSTADT, F. T.; BOERSMA, B. J.; WESTERWEEL, J. Turbulent diffusion. In: Turbulence. [S.1.]: Springer, 2016. p. 215-231.

OUELLETTE, N. T.; GOLLUB, J. P. Curvature fields, topology, and the dynamics of spatiotemporal chaos. Physical review letters, APS, v. 99, n. 19, p. 194502, 2007.

OVARLEZ, G.; COHEN-ADDAD, S.; KRISHAN, K.; GOYON, J.; COUSSOT, P. On the existence of a simple yield stress fluid behavior. Journal of Non-Newtonian Fluid Mechanics, Elsevier, v. 193, p. 68-79, 2013.

OVERMARS, E.; WARNCKE, N.; POELMA, C.; WESTERWEEL, J. Bias errors in piv: the pixel locking effect revisited. In: 15th international symposium on applications of laser techniques to fluid mechanics, Lisbon, Portugal. [S.1.: s.n.], 2010. p. 5-8.

OZALP, C.; PINARBASI, A.; FAKILAR, M.; SAHIN, B. Piv measurements of flow through a sudden contraction. Flow Measurement and Instrumentation, Elsevier, v. 18, n. 3-4, p. 121-128, 2007.

PALACIOS, F. Estudo experimental do escoamento de fluido newtoaniano em contração abrupta axissimétrica com a técnica de velocimetria por imagem de partículas. Federal University of Technology-Parana, 2011.

PALACIOS-MORALES, C.; ZENIT, R. The formation of vortex rings in shear-thinning liquids. Journal of Non-Newtonian Fluid Mechanics, Elsevier, v. 194, p. 1-13, 2013.

PALACIOS-MORALES, C.; ZENIT, R. Vortex ring formation for low re numbers. Acta Mechanica, Springer, v. 224, n. 2, p. 383-397, 2013. 
PEIXINHO, J.; NOUAR, C.; DESAUBRY, C.; THÉRON, B. Laminar transitional and turbulent flow of yield stress fluid in a pipe. Journal of Non-Newtonian Fluid Mechanics, Elsevier, v. 128, n. 2-3, p. 172-184, 2005.

PIAU, J. Carbopol gels: Elastoviscoplastic and slippery glasses made of individual swollen sponges: Meso-and macroscopic properties, constitutive equations and scaling laws. Journal of non-newtonian fluid mechanics, Elsevier, v. 144, n. 1, p. 1-29, 2007.

POUMAERE, A.; MOYERS-GONZÁLEZ, M.; CASTELAIN, C.; BURGHELEA, T. Unsteady laminar flows of a carbopol ${ }^{\circledR}$ gel in the presence of wall slip. Journal of Non-Newtonian Fluid Mechanics, Elsevier, v. 205, p. 28-40, 2014.

PRAGER, W. ON SLOW VISCO-PLASTIC FLOW. [S.1.], 1952.

PRITCHARD, P. J.; MITCHELL, J. W. Fox and McDonald's Introduction to Fluid Mechanics, Binder Ready Version. [S.1.]: John Wiley \& Sons, 2016.

RAFFEL, M.; WILLERT, C. E.; SCARANO, F.; KÄHLER, C. J.; WERELEY, S. T.; KOMPENHANS, J. Particle image velocimetry: a practical guide. [S.1.]: Springer, 2018.

RUDMAN, M.; BLACKBURN, H. M.; GRAHAM, L.; PULLUM, L. Turbulent pipe flow of shear-thinning fluids. Journal of non-newtonian fluid mechanics, Elsevier, v. 118, n. 1, p. 33-48, 2004.

SCHLICHTING, H.; GERSTEN, K. Fundamentals of boundary-layer theory. In: Boundary-Layer Theory. [S.1.]: Springer, 2017. p. 29-49.

SCHMIDT, M.; WASSNER, E.; MÜNSTEDT, H. Setup and test of a laser doppler velocimeter for investigations of flow behaviour of polymer melts. Mechanics of Time-Dependent Materials, Springer, v. 3, n. 4, p. 371-393, 1999.

SISAVATH, S.; JING, X.; PAIN, C. C.; ZIMMERMAN, R. W. Creeping flow through an axisymmetric sudden contraction or expansion. Journal of Fluids Engineering, American Society of Mechanical Engineers, v. 124, n. 1, p. 273-278, 2002.

SKELLAND, A. H. P. Non-Newtonian flow and heat transfer. [S.1.]: Wiley, 1967.

SOUZA-MENDES, P. R. de; NACCACHE, M. F.; VARGES, P. R.; MARCHESINI, F. H. Flow of viscoplastic liquids through axisymmetric expansions-contractions. Journal of Non-Newtonian Fluid Mechanics, Elsevier, v. 142, n. 1-3, p. 207-217, 2007.

STANISLAS, M.; WESTERWEEL, J.; KOMPENHANS, J. Particle image velocimetry: recent improvements. In: SPRINGER. Proceedings of the EUROPIV 2 Workshop. [S.1.], 2003. p. 145-161.

STEIN, E. Displacement of non-newtonian liquids in eccentric annuli. Master's Thesis, Rio de Janeiro, Brasil, Pontifícia Universidade Católica do Rio de Janeiro - PUC-RIO, 2005.

STRZELECKA, K.; JEZOWIECKA-KABSCH, K. Coriolis coefficient in transitional and turbulent pipe flow. OCHRONA SRODOWISKA, POLISH SANITARY ENGINEERS ASSOC UL MARSZ J PILSUDSKIEGO 74, WROCLAW, 2 SKR ..., v. 30, n. 1, p. 21-25, 2008.

SUNARSO, A.; YAMAMOTO, T.; MORI, N. Numerical analysis of wall slip effects on flow of newtonian and non-newtonian fluids in macro and micro contraction channels. Journal of fluids engineering, American Society of Mechanical Engineers, v. 129, n. 1, p. 23-30, 2007.

SZEWCZYK, H. Correction factors in one-dimensional flow pattern of a viscous incompressible fluid in a smooth circular pipe. Chemical And Process Engineering, v. 29, n. 1, p. 271-292, 2008. 
TAGHAVI, S.; ALBA, K.; MOYERS-GONZALEZ, M.; FRIGAARD, I. Incomplete fluid-fluid displacement of yield stress fluids in near-horizontal pipes: experiments and theory. Journal of Non-Newtonian Fluid Mechanics, Elsevier, v. 167, p. 59-74, 2012.

TANNER, R. I. Engineering rheology. [S.1.]: OUP Oxford, 2000. v. 52.

TOMITA, Y. On the fundamental formula of non-newtonian flow. Bulletin of JSME, The Japan Society of Mechanical Engineers, v. 2, n. 7, p. 469-474, 1959.

TOONDER, J. D.; NIEUWSTADT, F. Reynolds number effects in a turbulent pipe flow for low to moderate re. Physics of Fluids, AIP, v. 9, n. 11, p. 3398-3409, 1997.

TURIAN, R.; MA, T.-W.; HSU, F.-L.; SUNG, M.-J.; PLACKMANN, G. Flow of concentrated non-newtonian slurries: 2. friction losses in bends, fittings, valves and venturi meters. International journal of multiphase flow, Elsevier Science Publishing Company, Inc., v. 24, n. 2, p. 243-269, 1998.

VRADIS, G. C.; HAMMAD, K. J. Inertia effects on the flow of bingham plastics through sudden contractions in a pipe. The Canadian Journal of Chemical Engineering, Wiley Online Library, v. 74, n. 4, p. 457-463, 1996.

WERELEY, S. T.; MEINHART, C. D. Second-order accurate particle image velocimetry. Experiments in Fluids, Springer, v. 31, n. 3, p. 258-268, 2001.

WESTERWEEL, J. Theoretical analysis of the measurement precision in particle image velocimetry. Experiments in Fluids, Springer, v. 29, n. 1, p. S003-S012, 2000.

WESTERWEEL, J.; DRAAD, A.; HOEVEN, J. T. Van der; OORD, J. V. Measurement of fully-developed turbulent pipe flow with digital particle image velocimetry. Experiments in Fluids, Springer, v. 20, n. 3, p. 165-177, 1996.

ZHANG, X.-F.; ZHANG, Y.; LIU, L. Fluorescence lifetimes and quantum yields of ten rhodamine derivatives: structural effect on emission mechanism in different solvents. Journal of Luminescence, Elsevier, v. 145, p. 448-453, 2014. 


\section{APPENDIX A - PIV CALIBRATION TESTS}

Calibration test to set the parameters of this study, were carried out using water as Newtonian fluid. In order to perform these tests were evaluated different $R e$ at turbulent regime. Re is defined for a Newtonian fluid into circular tubes as:

$$
R e=\frac{\rho U D}{\mu}
$$

For the preliminary tests were used HGS particles and water-filled configuration for visualization box.

\section{A.1 Turbulent regime parameters}

Reynolds number was set for different values between 5000 and 30000. The acquisition parameters, post-process and vector calculation are presented at table A.1. In order to validate the results accuracy the velocity profiles and the first order turbulent quantities were compare with LDV and DNS data obtained in experimental and numerical studies carried out by Toonder and Nieuwstadt (1997) and Eggels et al. (1994).

For every turbulent $R e$ there is a data validation, for $R e=1000,17800$, and 24600 data were validated by LDV experimental study data. For $R e=5300$ also the values were compare with DNS data.

LDV is such a reliable technique for a comparison of the data obtained at the present study. Data obtained by this technique are chosen as validation data due to the accuracy of this technique, and the quantity of points that can be analyzed for each test.

For calibration tests were obtain the axial velocity $u$, and the velocity fluctuations $u^{\prime}$ and $v^{\prime}$. The root mean square (rms) of the axial and radial velocity represent the fluctuations of this velocities at turbulent regime. The turbulent regime is the most suitable region with high $R e$ to evaluate the PIV technique parameters. Thus increasing the Reynold numbers, the fluctuation increase in the whole section because the apparition of turbulent spots that can be visualized by PIV technique.

To obtain the normalized velocities, the velocity values were divide by the friction wall velocity $u_{*}$. Doorne and Westerweel (2007) expresses that for turbulent flow the friction velocity is defined by the friction factor, $f$ and the mean velocity $\bar{U}$ by:

$$
u_{*}=\bar{U} \sqrt{f / 8}
$$

where $f$ is given by the semi-empirical expression of Blasius:

$$
f_{\text {Blasius }}=0.316 R e^{-1 / 4}
$$

The friction velocity has a high sensitivity on the normalized velocity values, they have been calculated carefully to avoid systematic errors. For the range of Re studied, Westerweel et al. (1996) and Schlichting 
Table A.1 - Experimental parameters set for turbulent flow measurements.

\begin{tabular}{|c|c|c|}
\hline \multirow[t]{2}{*}{ Circular tube } & Diameter[mm] & 25,9 \\
\hline & Material & Acrylic \\
\hline \multirow[t]{4}{*}{ Flow properties } & Fluid & Water \\
\hline & Density $\left[\mathrm{kg} / \mathrm{m}^{3}\right]$ & $997.31,997.37,997.47,997.61$ \\
\hline & Dynamic viscosity $[\mathrm{mPa} \cdot \mathrm{s}]$ & $0.97,0.98,0.98,0.99$ \\
\hline & $R e$ & $5300,10000,17800,24600$ \\
\hline \multirow[t]{3}{*}{ Particle properties } & Material & HGS \\
\hline & Density $\left[\mathrm{g} / \mathrm{cm}^{3}\right]$ & 1.1 \\
\hline & Average diameter $[\mu \mathrm{m}]$ & 10 \\
\hline \multirow[t]{4}{*}{ Laser settings } & Type & Nd:YAG \\
\hline & Wavelength $[\mathrm{nm}]$ & 532 \\
\hline & Energy $[\mathrm{mJ}]$ & 60 \\
\hline & Laser beam height $[\mathrm{mm}]$ & 1 \\
\hline \multirow[t]{3}{*}{ Camera settings } & Type & CMOS \\
\hline & Resolution [Pixels] & $2532 \times 1728$ \\
\hline & Frequency $[\mathrm{Hz}]$ & 30 \\
\hline \multirow[t]{5}{*}{ Images } & Acquisition mode & Double frame \\
\hline & f-number & 8 \\
\hline & Visualization area $[\mathrm{mm} \times \mathrm{mm}]$ & $36 \times 26$ \\
\hline & Times between pulses $[\mu \mathrm{m}]$ & 300 \\
\hline & Pair of images & 1040 \\
\hline \multirow[t]{3}{*}{ PIV analysis } & Analysis mode & Bi-dimensional \\
\hline & Interrogation area [pixels] & $16 \times 16$ \\
\hline & Overlap & $50 \%$ \\
\hline
\end{tabular}

Source: Author (2019).

and Gersten (2017) recommend a experimental equation to determinate the wall friction velocity, given by:

$$
u_{*}=\sqrt{\frac{0.0791 R e^{-1 / 4}}{2}} \bar{U}
$$

equation (A.4) is more suitable to use for turbulent regimes thus the error for pressure measurements increases when the Eq. (A.2) is used.

Finally, the normalized axial velocity $U$, and the normalized velocity fluctuations $u^{\prime}$ and $v^{\prime}$ are described by:

$$
\begin{aligned}
& U^{+}=\frac{u}{u^{*}} \\
& u^{\prime+}=\frac{u^{\prime}}{u^{*}} \\
& v^{\prime+}=\frac{v^{\prime}}{u^{*}}
\end{aligned}
$$


Figure A.1 show the velocity profiles for Newtonian fluid at different $R e$ determined by PIV technique. The data have good accuracy with the LDV and DNS studies.

Figure A.1 - Normalized axial velocity profile for Newtonian fluid at turbulent Reynolds numbers.
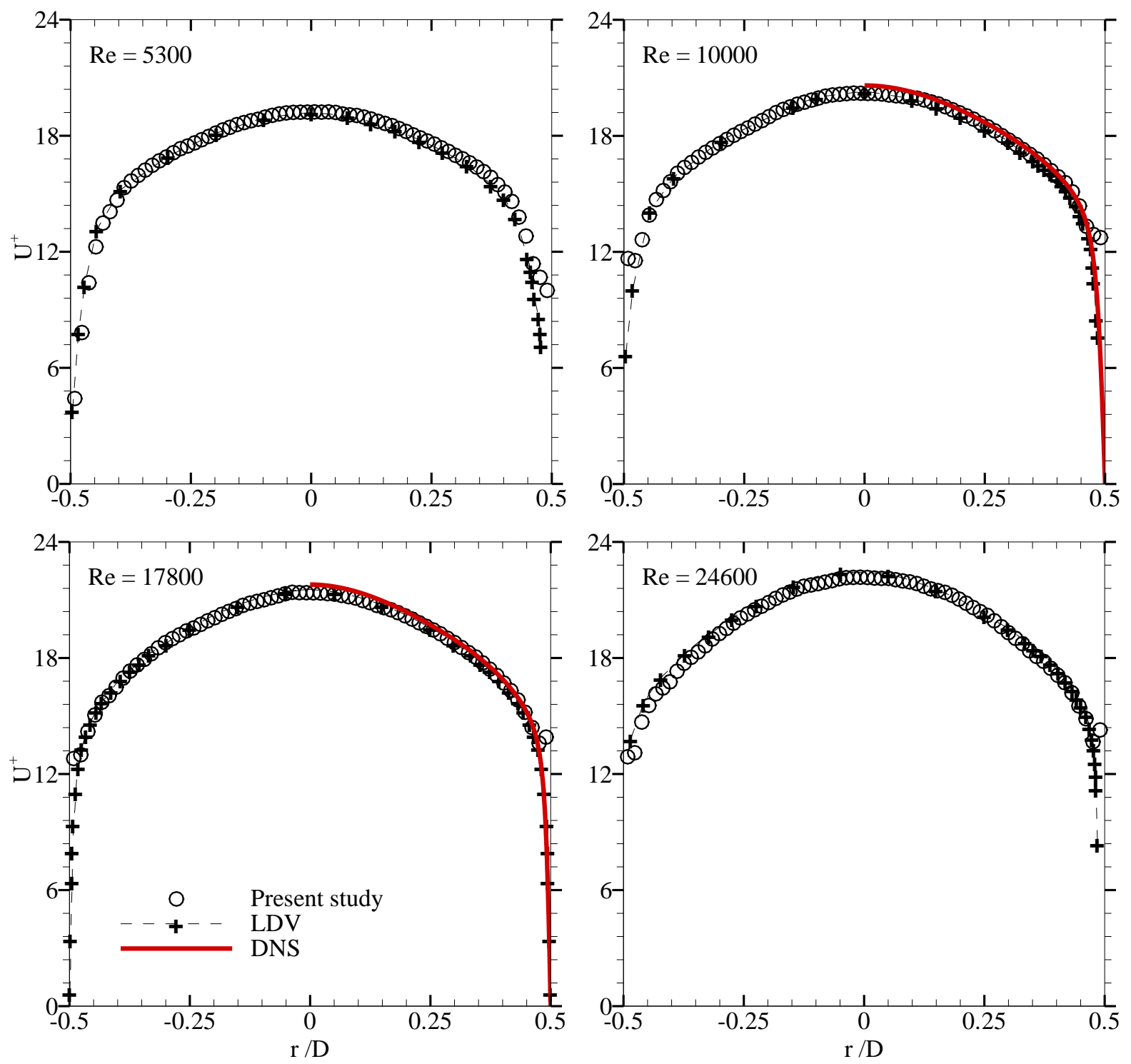

Source: Author (2019).

For $R e=10000,17800$ the DNS data have a deviation of $1.1 \%$ with the data obtained from the present study, because the DNS computations were performed for $R e=11700$ and 19000 respectively. Nevertheless, the Re set still being a good parameter for comparison of velocity profile and show an increasing tendency of the velocity profile as the $R e$ increases.

The normalized $r m s$ velocities were plotted and compared with DNS and LDV data. Some discrepancies in the region near to the wall, thus the light refracted distorts the images at larger velocities gradients. Nevertheless there is a good agreement between the experimental data obtained by PIV and LDV techniques.

The figure A.2 shows the fluctuations of normalized axial velocity, and figure A.3 presents the radial 
Figure A. 2 - Normalized axial velocity fluctuations, $u^{\prime+}$, as a function of the dimensionless wall distance $y^{+}$.
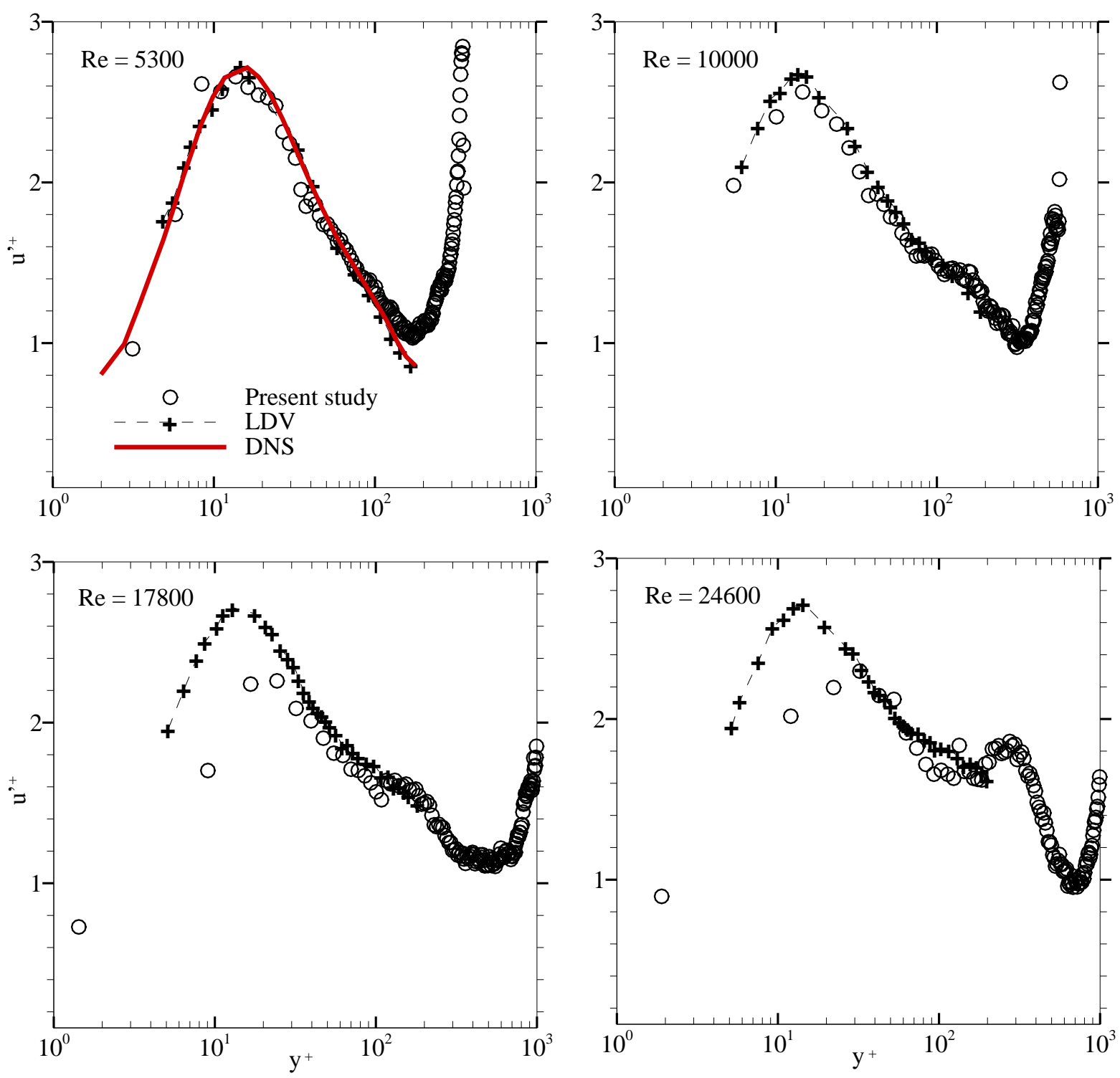

Source: Author (2019).

velocity profile fluctuations for the different turbulent $R e$ set for the Newtonian fluid tests.

Despite of there is a discrepancy from the $v^{\prime+}$ profiles, the behavior of the experimental data obtained in this study follow the LDV behavior. The accuracy of the data is due to the seeding particles and the refraction index at the wall pipe, it can be improved using seeding particles which work at high wavelength light as polyamide particles coated by rhodamine-B. For the first part of this experimental study, the data obtained are acceptable and shows that PIV technique is a good technique to correlate images in order to obtained mean and turbulent quantities for fluid flows. 
Figure A.3 - Normalized radial velocity fluctuations, $v^{\prime+}$, as a function of the dimensionless wall distance $y^{+}$.
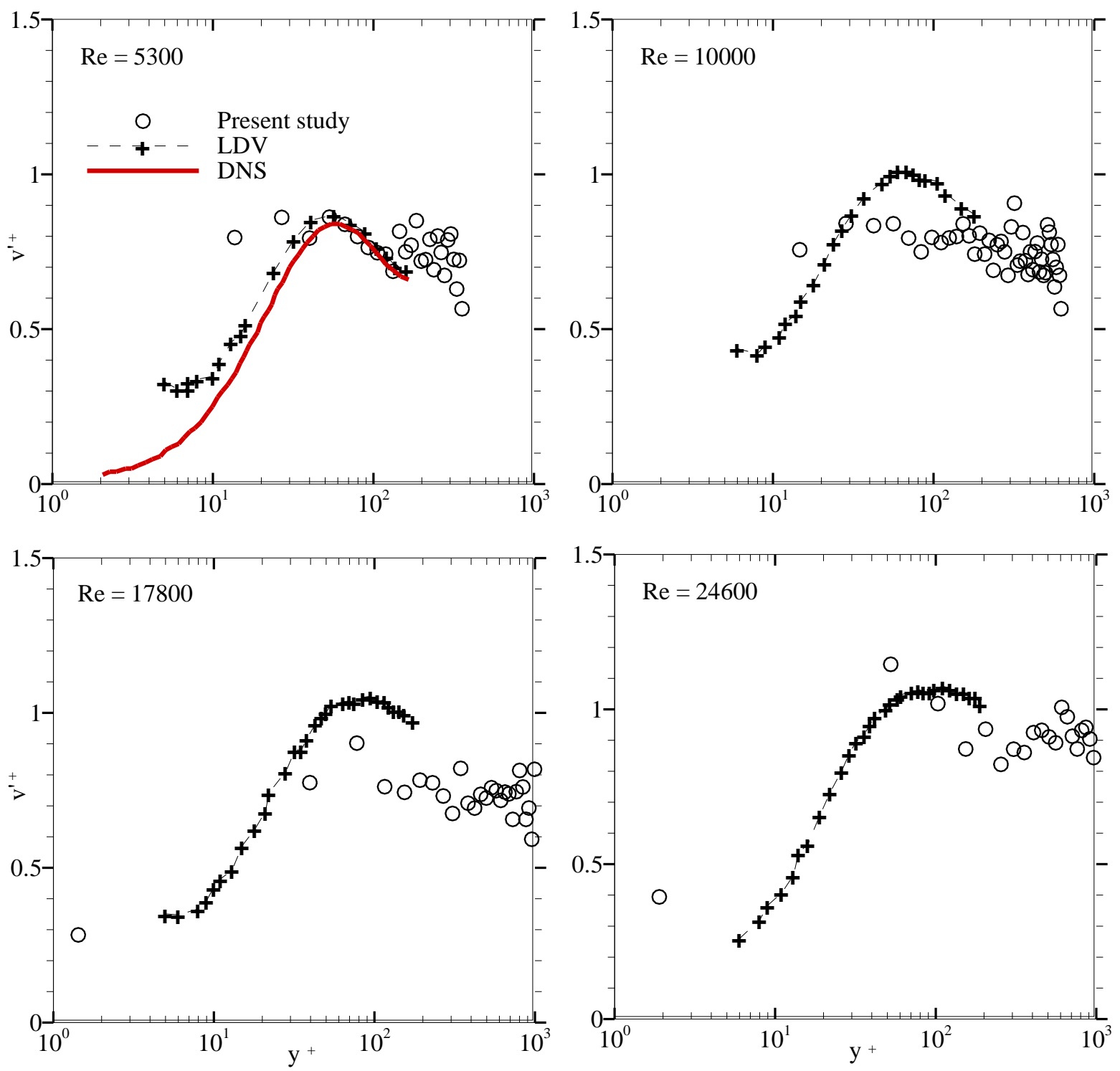

Source: Author (2019). 


\section{APPENDIX B - UNCERTAINTY ANALYSIS}

Based on definitions given in 4.6, in this appendix section are presented the uncertainty for pressure measurement and its derivate calculations.

\section{B.1 Pressure measurements and derivate quantities uncertainties}

The static pressure distribution in the upstream and downstream regions presents a linear trend between the measured points. For this reason, linear correlation criteria are used to calculate the upstream and downstream pressures at the contraction plane, $P_{c 1}$, and $P_{c 1}$, respectively.

The linear equation is obtained by the expression:

$$
Y=a X_{1}+b
$$

where $Y$ and $X_{1}$ are the dependent and the independent variables, respectively, $a$ is the slope of the linear function, and $b$ is its $y$-intercept. Then, the values for $a$ and $b$ are calculated using the least square method (COLEMAN; STEELE, 2018):

$$
\begin{gathered}
a=\frac{N_{X} \sum X_{1 i} Y_{i}-\sum X_{1 i} \sum Y_{i}}{N_{X} \sum\left(X_{1 i}^{2}\right)-\left(\sum X_{1 i}\right)^{2}} \\
b=\frac{\sum\left(X_{1 i}^{2}\right) \sum Y_{i}-\sum X_{1 i} \sum X_{1 i} Y_{i}}{N_{X} \sum\left(X_{1 i}^{2}\right)-\left(\sum X_{1 i}\right)^{2}}
\end{gathered}
$$

where $N_{X}$ is the number of points $\left(X_{1}, Y\right)$.

Then, using the expressions for linear correlation (COLEMAN; STEELE, 2018), the uncertainty calculated $b$ is given by:

$$
S_{b}=\left[S_{Y}^{2}\left(\frac{1}{N_{X}}+\frac{\left(\sum X_{1 i}\right)^{2}}{N_{X}^{2} S_{X X}}\right)\right]^{1 / 2}
$$

where $S_{Y}$ and $S_{X X}$ are defined as:

$$
\begin{gathered}
S_{Y}=\left\{\frac{\sum\left[Y_{i}-\left(a X_{1 i}+b\right)\right]^{2}}{N_{X}-2}\right\}^{1 / 2} \\
S_{X X}=\sum X_{1 i}^{2}-\frac{\left(\sum X_{1 i}\right)^{2}}{N_{X}}
\end{gathered}
$$

As presented in the subsection 5.1.1, the contraction plane is considered for the dimensionless axial distance $Z / D=0$ (ordinate axis), the pressures $P_{c 1}$ and ${ }_{c 2}$ can be interpreted as the $b$ values for two linear functions interpolated from pressure measurements along the the flow-loop.

Then the pressure $P_{c 1}$ and ${ }_{c 2}$ are calculated using the Eq. (B.3), and its uncertainty is calculated using Eq. (B.4) as $\delta P_{c 1}$ and $\delta P_{c 2}$ as a function of $R e_{D}^{\prime}$. Table summarized the values obtained for $P_{c 1}$ and $P_{c 2}$, and its uncertainty values. The uncertainty values and their equivalence in percentage (\%). 
Table B.1 - Uncertainty values of the extrapolated pressure $P_{c 1}$ and $P_{c 2}$.

\begin{tabular}{c|c|c|c|c|c|c|c}
\hline & $R e_{D}^{\prime}$ & $P_{c 1}[\mathrm{~Pa}]$ & $\delta P_{c 1}[\mathrm{~Pa}]$ & $\delta P_{c 1}(\%)$ & $P_{c 2}[\mathrm{~Pa}]$ & $\delta P_{c 2}[\mathrm{~Pa}]$ & $\delta P_{c 2}(\%)$ \\
\hline 10W & 1500 & 5105.93 & 11.63 & 0.23 & 4963.23 & 774.69 & 15.61 \\
& 2000 & 5107.16 & 4.39 & 0.09 & 4902.52 & 1641.611 & 33.49 \\
& 4000 & 6380.10 & 5.68 & 0.09 & 4968.36 & 1396.61 & 28.11 \\
& 5000 & 8411.11 & 16.52 & 0.20 & 5941.36 & 1024.88 & 17.25 \\
& 10000 & 23553.01 & 96.95 & 0.41 & 13474.55 & 2937.67 & 21.80 \\
\hline 20W & 10 & 8939.27 & 38.36 & 0.43 & 8692.35 & 857.07 & 9.86 \\
& 50 & 12559.78 & 14.27 & 0.11 & 11361.59 & 2903.19 & 25.55 \\
& 100 & 16241.47 & 6.33 & 0.04 & 14062.75 & 2909.53 & 20.69 \\
& 200 & 22415.56 & 51.83 & 0.23 & 18368.68 & 2925.53 & 15.93 \\
& 500 & 39104.17 & 20.84 & 0.05 & 28618.36 & 2953.61 & 10.32 \\
& 1000 & 74212.95 & 135.32 & 0.18 & 50683.87 & 3042.00 & 6.00 \\
& 1500 & 120992.39 & 133.23 & 0.11 & 80591.77 & 3125.32 & 3.88 \\
\hline $30 \mathrm{~W}$ & 10 & 17177.78 & 0.99 & $5.75 \mathrm{E}-3$ & 14347.61 & 2914.79 & 20.32 \\
& 50 & 27927.24 & 376.25 & 1.35 & 22407.67 & 2956.75 & 13.20 \\
& 100 & 36075.79 & 256.88 & 0.71 & 27730.83 & 2977.89 & 10.74 \\
& 200 & 47176.59 & 4.58 & $9.71 \mathrm{E}-3$ & 33571.96 & 3010.16 & 8.97 \\
& 300 & 60272.47 & 142.84 & 0.24 & 43430.42 & 3033.93 & 6.98 \\
& 500 & 103209.83 & 355.07 & 0.34 & 73757.95 & 3060.31 & 4.15 \\
& 650 & 127362.25 & 435.31 & 0.34 & 87715.20 & 3105.06 & 3.54 \\
\hline $40 \mathrm{~W}$ & 1 & 20861.93 & 18.17 & 0.08 & 19448.26 & 2930.29 & 15.07 \\
& 10 & 31535.80 & 71.62 & 0.23 & 26558.83 & 2973.91 & 11.20 \\
& 100 & 61172.09 & 494.05 & 0.81 & 44565.74 & 3059.87 & 6.87 \\
\hline
\end{tabular}

Source: Author (2019).

Calculating the arithmetic mean of the percentage uncertainty for $\delta P_{c 1}$ and $\delta P_{c 2}$ are obtained mean uncertainty of $0.29 \%$ and 14.07 , respectively. Therefore, it is observed that the higher values are obtained for the pressure at the downstream region, due to the slope, $m$, for the linear function is higher for the downstream pressures. Consequently, this leads to high errors for $P_{c 2}$.

By obtaining the pressures $P_{c 1}$ and $P_{c 2}$, and their associated uncertainties, the contraction pressure drop , $\Delta P_{c o n}$, and its uncertainty can be calculated for Reynolds number studied. As presented in section 4.7, $\Delta P_{c o n}$ is calculated by $\Delta P_{c o n}=P_{c 1}-P_{c 2}$, and its uncertainty is obtained by the expression $\delta \Delta P_{\text {con }}= \pm \sqrt{\delta P_{c 1}^{2}+\delta P_{c 2}^{2}}$.

Using the methodology described in subsection 4.6.1 and the expression (5.2) for the pressure loss coefficient at the contraction, $k_{\text {con }}$, it is possible to calculate the uncertainty for this coefficient $\delta k_{c o n}$. The velocities at the upstream and downstream regions can be expressed as a function of the mass flow rate with $Q=\bar{U}_{1} \pi D^{2} / 4=\bar{U}_{2} \pi d^{2} / 4$. Then, the Eq. (5.2) can be rewritten as:

$$
k_{\text {con }}=\frac{\pi^{2} d^{4} \Delta P_{\text {con }}}{8 \rho Q^{2}}+\alpha_{1} \frac{d^{4}}{D^{4}}-\alpha_{2}
$$

where the $\alpha_{1}$ and $\alpha_{2}$ are the kinetic energy correction coefficient. As expressed in 2.2, $\alpha_{1}$ and $\alpha_{2}$ reach 
values of 1 for laminar regime, and 2 for turbulent conditions. For turbulent regime the values of $\alpha_{1}$ and $\alpha_{2}$ are considered 1 due to the kinetic energy variation is generally small compared to the dominant terms in the energy equation (PRITCHARD; MITCHELL, 2016). These values area used as a simple way for calculating $\delta k_{c o n}$, and as stated by Fester et al. (2008), this consideration is also valid for non-Newtonian fluid flow.

Using Eq. (4.22), the uncertainty for pressure loss coefficient at the contraction is given by:

$$
\begin{aligned}
\delta k_{\text {con }}^{2}= & \left(\frac{\partial k_{\text {con }}}{\partial d}\right)^{2}(\delta d)^{2}+\left(\frac{\partial k_{\text {con }}}{\partial \Delta P_{\text {con }}}\right)^{2}\left(\delta \Delta P_{\text {con }}\right)^{2}+\left(\frac{\partial k_{\text {con }}}{\partial \rho}\right)^{2}(\delta \rho)^{2}+\left(\frac{\partial k_{\text {con }}}{\partial Q}\right)^{2}(\delta Q)^{2} \\
& +\left(\frac{\partial k_{\text {con }}}{\partial D}\right)^{2}(\delta D)^{2}
\end{aligned}
$$

where $\delta d, \delta \Delta P_{c o n}, \delta \rho, \delta Q$, and $\delta D$ are the uncertainty for the measurement of the upstream pipe diameter, pressure drop at the contraction, fluid density, volumetric flow, and downstream pipe diameter, respectively. Finding the partial derivative of the Eq. (B.7) for each variable and substituting in Eq. (B.8) is obtained a new expression for $\delta k_{\text {con }}$ :

$$
\begin{aligned}
\delta k_{\text {con }}^{2}= & \left(\frac{\pi^{2} \Delta P_{\text {con } d^{3}}}{2 \rho Q^{2}}+\alpha_{1} \frac{4 d^{3}}{D^{4}}\right)^{2}(\delta d)^{2}+\left(\frac{\pi^{2} d^{4}}{8 \rho Q^{2}}\right)^{2}\left(\delta \Delta P_{c o n}\right)^{2}+\left(-\frac{\pi^{2} \Delta P_{c o n} d^{4}}{8 \rho^{2} Q^{2}}\right)^{2}(\delta \rho)^{2} \\
& +\left(-\frac{\pi^{2} \Delta P_{\text {con }} d^{4}}{4 \rho Q^{3}}\right)^{2}(\delta Q)^{2}+\left(-4 \alpha_{1} \frac{d^{4}}{D^{5}}\right)^{2}(\delta D)^{2}
\end{aligned}
$$

In order to facilitate the calculations for $\delta k_{\text {con }}$, Eq. (B.9) can be simplified for the variable of interest. Therefore, the Eq. (B.9) can be rewritten as:

$$
\begin{aligned}
& \left(\frac{\delta k_{\text {con }}}{k_{\text {con }}}\right)^{2}=16\left(1+\frac{\alpha_{2}}{k_{\text {con }}}\right)^{2}\left(\frac{\delta d}{d}\right)^{2}+\left(1-\frac{\alpha_{1} / \beta^{4}-\alpha_{2}}{k_{\text {con }}}\right)^{2}\left(\frac{\delta \Delta P_{\text {con }}}{\Delta P_{\text {con }}}\right)^{2} \\
& +\left(1-\frac{\alpha_{1} / \beta^{4}-\alpha_{2}}{k_{\text {con }}}\right)^{2}\left(\frac{\delta \rho}{\rho}\right)^{2}+4\left(1-\frac{\alpha_{1} / \beta^{4}-\alpha_{2}}{k_{\text {con }}}\right)^{2}\left(\frac{\delta Q}{Q}\right)^{2}+16\left(\frac{\alpha_{1} / \beta^{4}}{k_{\text {con }}}\right)^{2}\left(\frac{\delta D}{D}\right)^{2}
\end{aligned}
$$

where $\beta$ is the contraction ratio given by $D / d$.

The values for the pipe diameters at the upstream and downstream region, $D=0.0259 \mathrm{~m}$ and $d=0.01215 \mathrm{~m}$, were measured with an uncertainty of $\delta D=\delta d= \pm 25 \mu \mathrm{m}$. The value of the uncertainty was obtained from the sensitivity specifications for the caliper divided by 2 (systematic bias). The sensitivity value for the caliper used is $\pm 50 \mu \mathrm{m}$.

The uncertainty values for the variables $Q$ and $\rho$ are determined considering the error resulting from the use of the mass flow meter. From the data provided by the manufacturer, the uncertainty for $Q$ and $\rho$ are estimated both in $\pm 0.2 \%$. In table B. 2 are presented the uncertainty values for $k_{\text {con }}$ calculated for each $R e_{D}^{\prime}$ and fluids.

From table B. 2 is obtained the arithmetic mean of the percentage uncertainty for $\delta k_{\text {con }}$, with a value of $32.59 \%$. It is observed that low values of $\delta k_{c}$ on are obtained for high $R e_{D}^{\prime}$ when the relative uncertainty for $\delta \Delta P_{\text {con }}$ is lower. The relative uncertainty $\delta k_{\text {con }}$ presents high values for the fluid $10 \mathrm{~W}$ for the $R e_{D}^{\prime}$ of 2000 and 4000 . This behavior for the uncertainty is explained due to these $R e_{D}^{\prime}$ are at the limit of the 
Table B.2 - Uncertainty values of $k_{\text {con }}$ for the different $R e_{D}^{\prime}$ and fluids.

\begin{tabular}{c|c|c|c|c|c|c|c|c|c}
\hline & $R e_{D}^{\prime}$ & $Q\left[\mathrm{~m}^{3} / \mathrm{s}\right]$ & $\rho\left[\mathrm{Kg} / \mathrm{m}^{3}\right]$ & $\Delta P_{\text {con }}[\mathrm{Pa}]$ & $\delta \Delta P_{\text {con }}$ & $\delta \Delta P_{\text {con }} \%$ & $k_{\text {con }}$ & $\delta k_{\text {con }}$ & $\delta k_{\text {con }} \%$ \\
\hline \multirow{7}{*}{ 10W } & 1500 & $6.66 \mathrm{E}-5$ & 996.97 & 142.70 & 27.65 & 19.47 & 0.37 & 0.23 & 62.45 \\
& 2000 & $9.01 \mathrm{E}-5$ & 996.84 & 204.58 & 68.51 & 33.49 & 0.10 & 0.31 & 308.59 \\
& 4000 & $1.77 \mathrm{E}-4$ & 996.75 & 1411.74 & 396.84 & 28.11 & 1.07 & 0.56 & 52.06 \\
& 5000 & $2.21 \mathrm{E}-4$ & 996.50 & 2469.75 & 426.06 & 17.25 & 1.36 & 0.39 & 28.84 \\
& 10000 & $4.36 \mathrm{E}-4$ & 996.35 & 10078.46 & 2197.10 & 21.80 & 1.51 & 0.53 & 34.97 \\
\hline \multirow{7}{*}{ 20W } & 10 & $2.31 \mathrm{E}-5$ & 997.66 & 246.92 & 24.37 & 9.87 & 20.25 & 2.19 & 10.80 \\
& 50 & $7.13 \mathrm{E}-5$ & 997.32 & 1198.19 & 306.14 & 25.55 & 9.44 & 2.88 & 30.50 \\
& 100 & $1.19 \mathrm{E}-4$ & 997.18 & 2178.72 & 450.78 & 20.69 & 5.52 & 1.52 & 27.54 \\
& 200 & $1.98 \mathrm{E}-4$ & 997.16 & 4046.88 & 644.67 & 15.93 & 3.15 & 0.79 & 25.18 \\
& 500 & $3.86 \mathrm{E}-4$ & 997.16 & 10485.81 & 1082.13 & 10.32 & 1.57 & 0.35 & 22.37 \\
& 1000 & $6.39 \mathrm{E}-4$ & 997.15 & 23529.08 & 1411.74 & 6.00 & 1.64 & 0.15 & 9.10 \\
& 1500 & $8.60 \mathrm{E}-4$ & 997.16 & 40400.62 & 1567.52 & 3.88 & 1.56 & 0.095 & 6.08 \\
\hline \multirow{7}{*}{$30 \mathrm{~W}$} & 10 & $4.52 \mathrm{E}-05$ & 997.65 & 2830.17 & 575.06 & 20.32 & 64.88 & 13.36 & 20.59 \\
& 50 & $1.35 \mathrm{E}-04$ & 997.77 & 5519.57 & 732.45 & 13.27 & 13.33 & 1.88 & 14.12 \\
& 100 & $2.20 \mathrm{E}-04$ & 997.66 & 8344.96 & 897.92 & 10.76 & 7.22 & 0.87 & 12.02 \\
& 200 & $3.53 \mathrm{E}-04$ & 997.54 & 13604.63 & 1220.33 & 8.97 & 4.19 & 0.45 & 10.78 \\
& 300 & $4.68 \mathrm{E}-04$ & 997.54 & 16842.05 & 1175.57 & 6.98 & 2.65 & 0.24 & 9.21 \\
& 500 & $6.64 \mathrm{E}-04$ & 997.44 & 29451.88 & 1225.20 & 4.16 & 2.15 & 0.13 & 5.88 \\
& 650 & $7.96 \mathrm{E}-04$ & 996.20 & 39647.05 & 1411.43 & 3.56 & 1.82 & 0.10 & 5.32 \\
\hline \multirow{6}{*}{$40 \mathrm{~W}$} & 1 & $1.82 \mathrm{E}-05$ & 997.79 & 1413.67 & 213.04 & 15.07 & 202.52 & 2.78 & 1.37 \\
& 10 & $7.13 \mathrm{E}-05$ & 997.90 & 4976.97 & 557.42 & 11.20 & 46.25 & 5.29 & 11.43 \\
& 100 & $3.18 \mathrm{E}-04$ & 997.75 & 16606.35 & 1149.13 & 6.92 & 7.56 & 0.58 & 7.73 \\
\hline \multirow{6}{*}{} & & & & & & & & &
\end{tabular}

Source: Author (2019).

transitional regime, where the stability of the flow is affected. Therefore, an unstable flow leads to static pressure fluctuations.

\section{B.2 PIV uncertainty}

For the vector maps obtained with the PIV technique is possible to calculate the error associated with the image acquisition. Based on the Westerweel et al. (1996) definitions, it is necessary to define an integral time scale and a measurement time. These variables are expressed as follow:

- Measurement time:

$$
M_{t}=\frac{X_{D} N_{T}}{\bar{U}}
$$

where $N_{T}$ is the total of images captured, $X_{D}$ is the length of the view size in the axial direction.

- Integral time scale:

$$
I_{t}=\frac{D}{\bar{U}}
$$

The measurement time and the integral time scale are the times estimated to reduce as much as 
possible the noise and the bias for the vector correlation for each pair of images. The measurement time is the time required to measure a single point during an interval of time; this variable is essential for measurements with LDV or LDA technique.

In the case of the PIV technique, the integral time scale is the key to set the time between pulses for each pair of images. The $I_{t}$ is the upper limit for the time between pulses in a pair of images. The time between pulses must necessarily be less than $I_{t}$ to guarantee a noise reduction in optimal correlation.

Table summarized the values calculated for $M_{t}$ and $I_{t}$ for the different Reynolds numbers set for each viscoplastic fluids.

Table B.3 - Values obtained for $M_{t}$ and $I_{t}$ as a function of the $R e_{D}^{\prime}$ for the different viscoplastic fluids.

\begin{tabular}{c|c|c|c|c}
\hline & $R e_{D}^{\prime}$ & $\bar{U}[\mathrm{~m} / \mathrm{s}]$ & $M_{t}[\mathrm{~s}]$ & $I_{t}[\mathrm{~s}]$ \\
\hline 10W & 1500 & 0.1263 & 426.4526 & 0.2050 \\
& 2000 & 0.1709 & 315.1343 & 0.1515 \\
& 4000 & 0.3361 & 160.2631 & 0.0770 \\
& 5000 & 0.4190 & 128.5705 & 0.0618 \\
& 10000 & 0.8266 & 65.1690 & 0.0313 \\
\hline 20W & 10 & 0.0438 & 1231.0713 & 0.5919 \\
& 50 & 0.1352 & 398.3355 & 0.1915 \\
& 100 & 0.2262 & 238.1807 & 0.1145 \\
& 200 & 0.3750 & 143.6452 & 0.0691 \\
& 500 & 0.7329 & 73.5086 & 0.0353 \\
& 1000 & 1.2137 & 44.3873 & 0.0213 \\
& 1500 & 1.6322 & 33.0060 & 0.0159 \\
\hline 30W & 10 & 0.0857 & 628.2556 & 0.3020 \\
& 50 & 0.2567 & 209.8378 & 0.1009 \\
& 100 & 0.4169 & 129.2041 & 0.0621 \\
& 200 & 0.6697 & 80.4402 & 0.0387 \\
& 300 & 0.8876 & 60.6955 & 0.0292 \\
& 500 & 1.2607 & 42.7325 & 0.0205 \\
& 650 & 1.5107 & 35.6599 & 0.0171 \\
\hline $40 \mathrm{~W}$ & 1 & 0.0345 & 1559.5994 & 0.7498 \\
& 10 & 0.1354 & 397.9791 & 0.1913 \\
& 100 & 0.6042 & 89.1542 & 0.0429 \\
\hline
\end{tabular}

Source: Author (2019).

High integral time scales are required for low $\bar{U}$ due to a longer time between frames is required for having a displacement of the seeding particle. Then, the values of $M_{t}$ and $I_{t}$ are used to calculate the error sampling for the current experimental setup, the error sampling is given by:

- Sampling error:

$$
e_{s}=\frac{1}{\sqrt{\frac{M_{t}}{I_{t}}}}
$$

Hence the values of $M_{t}$ and $t_{t}$ have a dependence of $\bar{U}$, the error sampling is strongly dependent on 
the geometrical aspect of the region visualized. So, $e_{s}$ is a function of $X_{D}$ and $D$ and for this study the value calculated is $e_{s}=2.19 \%$. This error value is small but not negligible, and it is important to know the percentage variation of the variables measured by the PIV technique. 